\title{
CHECK-LIST OF MOSSES OF EAST EUROPE AND NORTH ASIA
}

\section{СПИСОК МХОВ ВОСТОЧНОЙ ЕВРОПЫ И СЕВЕРНОЙ АЗИИ}

\author{
M.S. IGNATOV, O.M. AFONINA, E.A. IGNATOVA, \\ with contributions on regional floras from:
}

A. Abolina, T.V. AKATOVA, E. Z. BAisheVA, L.V. BARDUNOV, E.A. BARYAKINA, O.A. BELKINA, A.G. BEZGODOV, M.A.BOYCHUK, V.YA. CHERDANTSEVA, I.V. CZERNYADJEVA, G.YA. DOROSHINA, A.P. DYACHENKO, V.E. FEDOSOV, I.L. GOLDBERG, E.I. IVANOVA, I. JUKONIENE, L. KANNUKENE, S.G.KAZANOVSKY, Z.KH. KHARZINOV, L.E. KURBATOVA, A.I.MAKSIMOV, U.K. MAMATKULOVઐ, V. A. MANAKYAN↔, O.M. MASLOVSKY, M.G. NAPREENKO, T. N. OTNYUKOVA, L.YA. PARTYKA, O.YU. PISARENKO, N.N. POPOVA, G.F. RYKOVSKY, D.YA. TUBANOVA, G.V. ZHELEZNOVA, V.I. ZOLOTOV ${ }^{1}$

\section{М. С. ИГНАТОВ, О.М. АФОНИНА, Е.А. ИГНАТОВА, с дополнениями по флорам отдельных регионов:}

А.А. АБОЛИНЯ, Т.В. АКАТОВА, Э.З. БАИШЕВА, Л.В. БАРДУНОВ, Е.А. БАРЯКИНА, О.А. БЕЛКИНА, А.Г. БЕЗГОДОВ, М.А.БОЙЧУК, В.Я. ЧЕРДАНЦЕВА, И.В. ЧЕРНЯДЬЕВА, Г.Я. ДОРОШИНА, А.П. ДЬЯЧЕНКО, В.Э. ФЕДОСОВ, И.Л. ГОЛЬДБЕРГ, Е.И. ИВАНОВА, И. ЮКОНИЕНЕ, Л. КАННУКЕНЕ, С.Г. КАЗАНОВСКИЙ, 3.Х. ХАРЗИНОВ, Л.Е. КУРБАТОВА, А.И.МАКСИМОВ, У.К. МАМАТКУЛОВЪ, В. А. МАНАКЯНЪ, О.М. МАСЛОВСКИЙ, М.Г. НАПРЕЕНКО, Т. Н. ОТНЮКОВА, Л.Я. ПАРТЫКА, О.Ю. ПИСАРЕНКО, Н.Н. ПОПОВА, Г.Ф.РЫКОВСКИЙ, Д.Я. ТУБАНОВА, Г.В. ЖЕЛЕЗНОВА, В.И. ЗОЛОТОВ ${ }^{1}$

Abstract

Check-list of mosses of the East Europe and North Asia includes data on 1302 species, 8 subspecies and 42 varieties occurring in Lithuania, Latvia, Estonia, Belarus, Ukraine, Moldova, Russia, Georgia, Armenia, Azerbaijan, Kazakhstan, Turkmenistan, Uzbekistan, Kyrgyzstan, and Tadjikistan. Territories of Ukraine and Russia are subdivided into 3 and 19 regions respectively. Each record for a country and region has a reference to the relevant publication.

Резюме

Список мхов Восточной Европы и Северной Азии включает сведения о 1302 видах, 8 подвидах и 42 разновидностях мхов, встречающихся в Литве, Латвии, Эстонии, Беларуси, Украине, Молдове, России, Грузии, Армении, Азербайджане, Казахстане, Туркменистане, Узбекистане, Киргизии, Таджикистане. Территории Украины и России подразделены дополнительно на 3 и 19 регионов соответственно. Все указания на нахождение вида в странах или регионах сопровождаются ссылками на соответствующие источники информации.

Comb. nov.: Claopodium rostratum (Hedw.) Ignatov, Dicranella curvipes (Lindb.) Ignatov, Hygrohypnella bestii (Renauld \& Bryhn) Ignatov \& Ignatova, Lindbergia grandiretis (Lindb. ex Broth.) Ignatov \& Ignatova, Ochyraea duriuscula (De Not.) Ignatov \& Ignatova, Sciuro-hypnum altaicum (Ignatov) Ignatov, Stereodon subimponens var. ulophyllum (Müll. Hal.) Afonina.

1 - Author addresses are given in the end of the paper. - Адреса авторов даны в конце статьи. 


\section{INTRODUCTION}

In 1992 the first check-list of mosses of the territory of East Europe and North Asia was published (Ignatov \& Afonina, 1992). Check-lists of this kind become out of date within 10-15 years, as can be seen from the history of checklists of Europe (Corley \& al., 1981; Corley \& Crundwell, 1991; Hill \& al., 2006), Japan (Iwatsuki \& Noguchi, 1973; Iwatsuki, 1991; Iwatsuki, 2004), China (Redfearn \& Wu, 1986; Redfearn \& al., 1996), North America ( Crum \& al., 1979; Anderson \& al., 1990), etc. Correspondingly, the first check-list of East Europe and North Asia also became out of date and therefore has to be revised. Mistakes in the first check-list were found to be rather numerous, urging us to correct them in a publication covering preferably the same territory.

Recent decades were quite fruitful for bryology in the states of the East Europe and North Asia. Descriptive floras and handbooks were published for Estonia (Ingerpuu \& al., 1998), Lithuania (Jukoniene, 2003), Belarus (Rykovsky \& Maslovsky, 2004), Karelia (Abramov \& Volkova, 1998), Middle European Russia (Ignatov \& Ignatova, 20032004); four-volumed "Moss flora of Ukraine" (Bachurina \& Melnichuk, 1987-1989, 2003) has been completed; the first volume of "Moss flora of Tadjikistan" (Mamatkulov, 1990) was published. New check-lists, conspectes and annotated lists appeared for many large areas: Eurasian Arctic (Afonina \& Czernyadjeva, 1995), Karelia (Volkova \& Maksimov, 1993), Komi (Zheleznova, 1994), Urals (Dyachenko, 1997), Central Russian Upland (Popova, 2002), West Siberia (Lapshina \& Muldiyarov, 1998), Siberia as a whole (Bardunov, 1992), Yakutia (Ivanova \& al., 2005), Kamchatka (Czernyadjeva, 2005), Chukotka (Afonina, 2004a), Ukraine (Virchenko, 2000, 2001; Virchenko \& Váňa, 2000), Georgia (Chikovani \& Svanidze, 2004), Armenia (Manakyan, 1995), Kabardino-Balkaria (Kharzinov \& al., 2004), Crimea (Partyka, 2005), Middle Asia (Mamatkulov \& al., 1998). Important contributions were published in species lists of provinces and nature reserves.

Species are annotated with their distribution up to the country level, except Ukraine (subdivided into 3 regions: Carpathians, main lowland territory and Crimea), and Russia (subdivided into 19 regions, cf. Fig. 1, Table 1 ).

\section{ВВЕДЕНИЕ}

В 1992 году был издан первый чеклист мхов территории Восточной Европы и Северной Азии (Ignatov \& Afonina, 1992). Как показывает история, такого рода работы устаревают за 10-15 лет. Это можно видеть напримерах чеклистов мхов Европы (Corley \& al., 1981; Corley \& Crundwell, 1991; Hill \& al., 2006), Японии (Iwatsuki \& Noguchi, 1973; Iwatsuki, 1991; Iwatsuki, 2004), Китая (Redfearn \& Wu, 1986; Redfearn \& al., 1996), Северной Америки (Crum \& al., 1979; Anderson \& al., 1990) и др. Естественно, что и первый чеклист Восточной Европы и Северной Азии уже сильно устарел и требует переиздания. Ошибки, допущенные в нем, оказались весьма многочисленными, и исправлять их, конечно, целесообразно в работе, охватывающей ту же самую территорию.

Последние десятилетия стали очень продуктивными в бриофлористике данной территории. Были изданы иллюстрированные флоры и определители Эстонии (Ingerpuu \& al., 1998), Литвы (Jukoniene, 2003), Беларуси (Рыковский, Масловский, 2004), Карелии (Абрамов, Волкова, 1998), средней части Европейской России (Игнатов, Игнатова, 2003-2004), завершено издание четырехтомной «Флоры листяних мохів Украіни» (Бачурина, Мельничук, 1987-1989, 2003), издан первый том флоры мхов Таджикистана (Маматкулов, 1990). Чеклисты, конспекты и аннотированные списки видов были опубликованы по многим крупным регионам: Арктике Евразии (Afonina \& Czernyadjeva, 1995), Карелии (Волкова, Максимов, 1993), Коми (Железнова, 1994), Уралу (Дьяченко, 1997), Среднерусской возвышенности (Попова, 2002), Западной Сибири (Lapshina \& Muldiyarov, 1998), Сибири в целом (Бардунов, 1992), Якутии (Иванова и др., 2005), Камчатке (Czernyadjeva, 2005), Чукотке (Афонина, 2004a), Украине (Вірченко, 2000, 2001; Вірченко, Ваня, 2000), Грузии (Chikovani \& Svanidze, 2004), Армении (Манакян, 1995), Кабардино-Балкарии (Kharzinov \& al., 2004), Крыму (Партыка, 2005), странам Средней Азии (Маматкулов и др., 1998). Большой вклад внесли каталоги мхов отдельных областей и республик, а также заповедников.

Для видов указано распространение с точностью до страны, кроме Украины, которая подразделяется на Карпаты, основную равнинную территорию и Крым, и России, в которой выделяется 19 регионов (Рис. 1, Табл. 1). 
Unlike the first check-list, each record for a particular region has a reference to the relevant literature source. Doing this, we include only the most comprehensive publication(s): if a recent checklist for a region exists it was the only publication cited. Most of them are included in the Table 1.

There are a lot of records scattered in numerous publications, which we tried to get together in the present checklist. We faced also a problem with the numerous herbarium specimens, quite important, but still unpublished. By agreement with the regional contributors, most of these collections were presented in this volume of Arctoa in the section "New Records", which, we hope, will speed up the presenting of interesting and valuable findings.

The wide usage of DNA data in systematics in the last decade has brought a lot of changes in the understanding of moss phylogeny, resulting in alterations in moss taxonomy and nomenclature and especially in those of pleurocarps. This checklist uses mostly the system of Goffinet \& Buck (2004), with the alterations for pleurocarps of Ignatov \& Ignatova (2004). The latter changes are commented on by Ignatov \& al. (2006), and they are mostly not repeated in the present paper.

Author citation follows mostly Brummit \& Powell (1992). The use of 'in' to indicate description of a taxon by one author in a paper published by another author is not used here. This style was widely used in 1990s, including the first checklist (Ignatov \& Afonina, 1992), but brought nothing but inconvenience of citations. We agree that the 'in' has to be restricted to the nomenclature citation, but not be included in the ordinary text.

The citation of authors of "Bryologia Europaea" is still problematic and incongruent between authors. We continue to attribute the autorship of the book to all three authors, citing them Bruch et al. (equivalent to B.S.G.), in view of no internal evidence to restrict the authorship to one or two of them.

\section{ACKNOWLEDGEMENTS}

We are grateful to Angela Newton for her linguistic correction of the manuscript. The work was partly supported by RFBR grants 04-0448774, 05-04-48705, Program Biodiversity of Russian Academy of Sciences, and by Scientific School Program HШ-7063.2006.4.
В отличие от первого чеклиста, все указания на нахождение вида в том или ином районе имеют ссылку на публикацию(и), обычно наиболее представительную(ые) для региона (при наличии недавней сводки мы ею одной и ограничивались). Список таких работ дан в таблице 1 .

Масса сведений о находках видов рассеяна в многочисленных публикациях, которые мы постарались суммировать в чеклисте. Неопубликованные ранее гербарные сборы, среди которых много интересных коллекций, в значительной степени представлены в данном томе в рубрике “Новые находки", которая, мы надеемся, позволит и в дальнейшем ускорить публикацию интересных флористических новинок.

Масштабные исследования ДНК внесли в последнее десятилетие значительные изменения в представление об эволюции мхов. Это повлияло и на систематику, и на номенклатуру, в особенности бокоплодных мхов. В настоящем чеклисте мы следуем преимущественно системе Goffinet \& Buck (2004), а для бокоплодных - с изменениями Игнатова и Игнатовой (2004). Обсуждение данного вопроса дано Ignatov \& al. (2006) и здесь не комментируется.

Цитирование авторов дается по Brummit \& Powell (1992). Факт описания таксона одним автором в работе другого, влекущий 'in' между авторами, опускается - этот необходимый элемент цитирования авторства нужен для номенклатурных цитат, но использование его при обычном цитировании авторов излишне (такая практика появилась в 1990-х годах и первый чеклист ей следовал, но ни к чему, кроме излишней громоздкости, она не привела).

Цитирование "Bryologia Europaea" продолжает оставаться неоднозначным и создавать дополнительные проблемы. В данной версии чеклиста мы используем Bruch et al. (как полный эквивалент B.S.G.), не считая обоснованным приписывать авторство тех или иных видов одному или двум авторам этой книги.

\section{БЛАГОДАРНОСТИ}

Мы благодарны Анджеле Ньютон за правку английского языка рукописи. Работа была частично поддержана грантами РФФИ 04-0448774, 05-04-48705, Программой "Биоразнообразие" Президиума РАН и Программой поддержки научных школ НШ-7063.2006.4. 


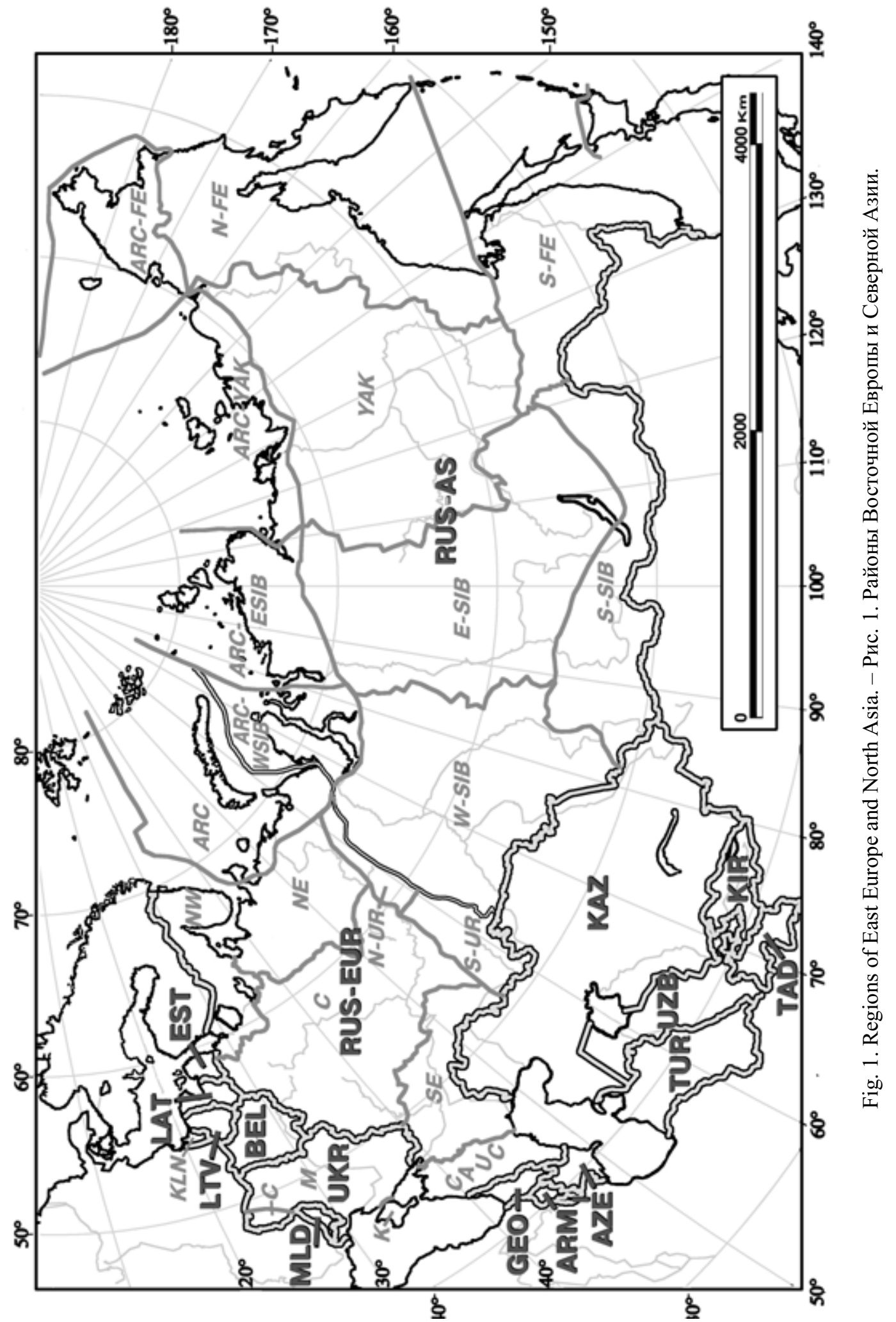


Table 1. Abbreviation of regions in the present check-list (cf. Fig. 1) and main sources of the regional information.Табл. 1. Сокращения районов чеклиста (см. Рис. 1) и основные источники информации по их флорам мхов.

Region abbreviation Region

$\begin{array}{ll}\text { LTV } & \text { Lithuania (Liet } \\ \text { LAT } & \text { Latvia } \\ \text { EST } & \text { Estonia } \\ \text { BEL } & \text { Belarus } \\ \text { UKR (C) } & \text { Ukraine, Carpa } \\ \text { UKR (M) } & \text { Ukraine, mainl } \\ \text { UKR (K) } & \text { Ukraine, Crime } \\ \text { MLD } & \text { Moldova } \\ \text { RUS-EUR (ARC) } & \text { Russian Europ } \\ & \text { including Polar } \\ \text { RUS-EUR (NW) } & \text { Russian Europe } \\ & \text { [Murmansk, Kar } \\ \text { RUS-EUR (NE) } & \text { Russian Europ } \\ & \text { [Arkhangelsk, low } \\ \text { RUS-EUR (KLN) } & \text { Kaliningrad Pro } \\ \text { RUS-EUR (C) } & \text { Russian European } \\ \text { RUS-EUR (N-UR) } & \text { Subpolar and Nor } \\ \text { RUS-EUR (S-UR) } & \text { Middle and So } \\ \text { RUS-EUR (SE) } & \text { Russian Europe } \\ \text { RUS-EUR (CAUC) } & \text { Russian part of Ca } \\ & \\ \text { GEO } & \text { Georgia } \\ \text { ARM } & \text { Armenia } \\ \text { AZE } & \text { Azerbaijan } \\ \text { KAZ } & \text { Kazakhstan } \\ \text { TUR } & \text { Turkmenistan } \\ \text { UZB } & \text { Uzbekistan } \\ \text { TAD } & \text { Tadjikistan } \\ \text { KYR } & \text { Kyrgyzstan } \\ \text { RUS-AS(ARC-WSB } & \text { Arcic West }\end{array}$

RUS-AS (ARC-WSIB) Arctic West Siberia

RUS-AS (ARC-ESIB) Arctic East Siberia

RUS-AS (W-SIB) West Siberia (cf. South Siberia)

RUS-AS (S-SIB) South Siberia ${ }^{2}$

RUS-AS (E-SIB)

RUS-AS (ARC-YAK) Arctic Yakutia

RUS-AS (YAK) Yakutia

RUS-AS (ARC-FE) Arctic Far East

RUS-AS (N-FE) North Far East

RUS-AS (S-FE) South Far East
Main source(s) of information

Jukoniene, 2003

Abolina, 2001

Ingerpuu \& al., 1998

Rykovsky \& Maslovsky, 2004

Zerov \& Partyka, 1975; Bachurina \& Melnichuk, 1987-1989,2003

Zerov, 1964; Bachurina \& Melnichuk, 1987-1989, 2003

Partyka, 2005

Simonov, 1978

Afonina \& Czernyadjeva, 1995; Dyachenko, 1997 


\section{Systematic ArRangement}

Systematic arrangement follows Goffinet \& Buck (2004), with some alterations according to Ignatov \& Ignatova (2004), Ignatov, Gardiner \& al. (2006), and Ochyra \& al. (2004). Asterisk $\left(^{*}\right)$ before some generic names means that their position in respective family is not very well proved.

SPHAGNOPSIDA Schimp.

SPHAGNALES C. Martius

Sphagnaceae Martynov

Sphagnum L.

ANDREAEOPSIDA Trevisan

ANDREAEALES Prantl

Andreaeaceae Dumort. Andreaea Hedw.

OEDIPODIOPSIDA (Schimp.) Goffinet \& W.R.Buck

OEDIPODIALES (Schimp.) Goffinet \& W.R.Buck

Oedipodiaceae Schimp.

Oedipodium Schwägr.

POLYTRICHOPSIDA Ochyra, Żarnowiec \& Bednarek-Ochyra

POLYTRICHALES M.Fleisch.

Polytrichaceae Schwägr.

Atrichum P.Beauv.

Bartramiopsis Kindb.

Lyellia R.Br.

Oligotrichum DC.

Pogonatum P.Beauv.

Polytrichastrum G.L.Sm.

Polytrichum Hedw.

Psilopilum Brid.

TETRAPHIDOPSIDA (M.Fleisch.) Goffinet \& W.R.Buck

TETRAPHIDALES M.Fleisch.

Tetraphidaceae Schimp.

Tetraphis Hedw.

Tetrodontium Schwägr.

BRYOPSIDA Horan.

BUXBAUMIALES M.Fleisch.

Buxbaumiaceae Schwägr.

Buxbaumia Hedw.

DIPHYSCIALES M.Fleisch.

Diphysciaceae M.Fleisch.

Diphyscium D.Mohr

TIMMIALES (M.Fleisch.) Ochyra

Timmiaceae Schimp.

Timmia Hedw.
CATOSCOPIALES Ignatov \& Ignatova

Catoscopiaceae Boulay ex Broth.

Catoscopium Brid.

FUNARIALES M.Fleisch.

Disceliaceae Schimp.

Discelium Brid.

Funariaceae Schwägr.

Entosthodon Schwägr.

Funaria Hedw.

Physcomitrella Bruch et al.

Physcomitrium (Brid.) Brid.

Pyramidula Brid.

ENCALYPTALES Dixon

Encalyptaceae Schimp.

Bryobrittonia R.S.Williams

Encalypta Hedw.

BRYOXIPHIALES H.A.Crum \& L.E.Anderson

Bryoxiphiaceae Besch.

Bryoxiphium Mitt.

SCOULERIALES (S.P.Churchill) Goffinet \& W.R.Buck

Drummondiaceae (Vitt) Goffinet

Drummondia Hook.

Scouleriaceae S.P.Churchill

Scouleria Hook.

GRIMMIALES M.Fleisch.

Grimmiaceae Arn.

Bucklandiella Roiv.

Codriophorus P.Beauv.

Coscinodon Spreng.

Grimmia Hedw.

Indusiella Broth. \& Müll.Hal.

Jaffueliobryum Thér.

Niphotrichum (Bednarek-Ochyra)

Bednarek-Ochyra \& Ochyra

Racomitrium Brid.

Schistidium Bruch et al.

Seligeriaceae Schimp.

Brachydontium Fürnr.

Blindia Bruch et al.

Seligeria Bruch et al.

Ptychomitriaceae Schimp.

Campylostelium Bruch et al.

Ptychomitrium Fürnr.

*Glyphomitrium Brid. 
Archidiaceae Schimp. Archidium Brid.

DICRANALES H.Philib. ex M.Fleisch.

Leucobryaceae Schimp.

Brothera Müll.Hal.

Campylopus Brid.

Dicranodontium Bruch et al.

Leucobryum Hampe

Bruchiaceae Schimp.

Trematodon Michx.

Dicranaceae Schimp.

*Aongstroemia Bruch et al.

*Dicranella (Müll.Hal.) Schimp.

Dicranoloma (Renauld) Renauld

Dicranum Hedw.

Paraleucobryum (Limpr.) Loeske

Rhabdoweisiaceae Limpr.

Amphidium Schimp.

Arctoa Bruch et al.

Cnestrum I.Hagen

Cynodontium Bruch et al.

Dichodontium Schimp.

Dicranoweisia Milde

Hymenoloma Ochyra

Kiaeria I.Hagen

Oncophorus (Brid.) Brid.

Oreas Brid.

Rhabdoweisia Bruch et al.

Ditrichaceae Limpr.

Ceratodon Brid.

Cleistocarpidium Ochyra \&

Bednarek-Ochyra

Distichium Bruch et al.

Ditrichum Timm ex Hampe

Pleuridium Rabenh.

Pseudephemerum (Lindb.) I.Hagen

Saelania Lindb.

Pottiaceae Schimp.

Acaulon Müll.Hal.

Aloina Kindb.

Anoectangium Schwägr.

Barbula Hedw.

Bryoerythrophyllum P.C.Chen

Cinclidotus P.Beauv.

Crossidium Jur.

Crumia W.B.Schofield

Didymodon Hedw.

Eucladium Bruch et al.

Gymnostomum Nees \& Hornsch.
Gyroweisia Schimp.

Hennediella Paris

Hilpertia R.H.Zander

Hydrogonium (Müll.Hal.) A.Jaeger

Hymenostylium Brid.

Hyophila Brid.

Leptobarbula Schimp.

Leptodontium (Müll.Hal.) Lindb.

Microbryum Schimp.

Molendoa Lindb.

Oxystegus (Lindb. ex Limpr.) Hilp.

Pleurochaete Lindb.

Pseudocrossidium R.S.Williams

Pterygoneurum Jur.

Scopelophila (Mitt.) Lindb.

Stegonia Venturi

Syntrichia Brid.

Timmiella (De Not.) Schimp.

Tortella (Müll.Hal.) Limpr.

Tortula Hedw.

Trichostomum Bruch

Weissia Hedw.

Ephemeraceae Schimp.

Ephemerum Hampe

Fissidentaceae Schimp.

Fissidens Hedw.

Schistostegaceae Schimp.

Schistostega D.Mohr

SPLACHNALES (M.Fleisch.) Ochyra

Meesiaceae Schimp.

Amblyodon P.Beauv.

Leptobryum (Bruch et al.) Wilson

Meesia Hedw.

Paludella Brid.

Splachnaceae Grev. \& Arn.

Aplodon R.Br.

Splachnum Hedw.

Tayloria Hook.

Tetraplodon Bruch et al.

Voitia Hornsch.

ORTHOTRICHALES Dixon

Orthotrichaceae Arn.

Macromitrium Brid.

Orthotrichum Hedw.

Ulota D.Mohr

Zygodon Hook. \& Taylor

HEDWIGIALES Ochyra

Hedwigiaceae Schimp.

Hedwigia P.Beauv. 
BRYALES Limpr.

Bryaceae Schwägr.

Anomobryum Schimp.

Brachymenium Schwägr.

Bryum Hedw.

Plagiobryum Lindb.

Rhodobryum (Schimp.) Limpr.

Orthodontiaceae (Broth.) Goffinet

Orthodontium Schwägr.

Orthodontopsis Ignatov \& B.C.Tan

Mielichhoferiaceae Schimp.

Epipterygium Lindb.

Mielichhoferia Nees \& Hornsch.

Pohlia Hedw.

Mniaceae Schwägr.

Cinclidium Sw.

Cyrtomnium Holmen

Mnium Hedw.

Plagiomnium T.J.Kop.

Pseudobryum (Kindb.) T.J.Kop.

Rhizomnium (Broth.) T.J.Kop.

Trachycystis T.J.Kop.

Bartramiaceae Schwägr.

Anacolia Schimp.

Bartramia Hedw.

Conostomum Sw. ex F.Weber \& D.Mohr

Philonotis Brid.

Plagiopus Brid.

Aulacomniaceae Schimp.

Aulacomnium Schwägr.

HOOKERIALES M.Fleisch.

Hookeriaceae Schimp. Hookeria Sm.

Hypopterygiaceae Mitt. Hypopterygium Brid.

HYPNALES Dumort.

Fontinalaceae Schimp.

Dichelyma Myrin

Fontinalis Hedw.

Fabroniaceae Schimp.

Fabronia Raddi

Habrodontaceae Rabenh.

Habrodon Schimp.

Plagiotheciaceae (Broth.) M.Fleisch.

Bardunovia Ignatov \& Ochyra

Herzogiella Broth.

Isopterygiopsis Z.Iwats.

Myurella Bruch et al.

Orthothecium Bruch et al.

Plagiothecium Bruch et al.
Platydictya Berk.

*Pseudotaxiphyllum Z.Iwats.

Struckia Müll.Hal.

Pterigynandraceae Schimp.

Pterigynandrum Hedw.

Leucodontaceae Schimp.

Dozya Sande Lac.

Leucodon Schwägr.

Cryphaeaceae Schimp.

Cryphaea D.Mohr

Hypnaceae Martynov

Eurohypnum Ando

Hypnum Hedw.

*Hondaella Dixon \& Sakurai

*Leptopterigynandrum Müll.Hal.

*Schwetschkeopsis Broth.

*Taxiphyllum M.Fleisch.

*Vesicularia (Müll.Hal.) Müll.Hal.

Entodontaceae Kindb.

Entodon Müll.Hal.

Pylaisiadelphaceae Goffinet \& W.R.Buck

Brotherella Loeske ex M.Fleisch.

*Fauriella Besch.

Heterophyllium (Schimp.) Kindb.

Platygyrium Bruch et al.

Pylaisiadelpha Cardot

Pseudoleskeaceae Schimp.

Lescuraea Bruch et al.

Rigodiadelphus Dixon

Anomodontaceae Kindb.

Anomodon Hook. \& Taylor

Haplohymenium Dozy \& Molk.

Herpetineuron (Müll.Hal.) Cardot

Heterocladiaceae Ignatov \& Ignatova

Heterocladium Bruch et al.

Neckeraceae Schimp.

Forsstroemia Lindb.

Homalia Brid.

*Homaliadelphus Dixon \& P.de la Varde

Leptodon D.Mohr

Neckera Hedw.

Thamnobryum Nieuwl.

Climaciaceae Kindb.

Climacium F.Weber \& D.Mohr

Pleuroziopsis Kindb. ex E.Britton

Antitrichiaceae Ignatov \& Ignatova Antitrichia Brid.

Hylocomiaceae (Broth.) M.Fleisch.

*Ctenidium (Schimp.) Mitt.

Hylocomiastrum Broth. 
Hylocomiopsis Cardot

Hylocomium Bruch et al.

Pleurozium Mitt.

Loeskeobryum Broth.

Rhytidiadelphus (Limpr.) Warnst.

Pterogoniaceae Rabenh.

Pterogonium Sw.

Lembophyllaceae Broth.

Dolichomitriopsis S.Okamura

Isothecium Brid.

Meteoriaceae Kindb.

Meteorium (Brid.) Dozy \& Molk.

Brachytheciaceae Schimp.

Brachytheciastrum Ignatov \& Huttunen

Brachythecium Bruch et al.

Bryhnia Kaurin

Cirriphyllum Grout

Eurhynchiadelphus Ignatov \& Huttunen

Eurhynchiastrum Ignatov \& Huttunen

Eurhynchium Bruch et al.

Homalothecium Bruch et al.

Kindbergia Ochyra

Myuroclada Besch.

Okamuraea Broth.

Oxyrrhynchium (Schimp.) Warnst.

Palamocladium Müll.Hal.

Plasteurhynchium M.Fleisch. ex Broth.

Pseudoscleropodium (Limpr.) M.Fleisch.

Rhynchostegiella (Schimp.) Limpr.

Rhynchostegium Bruch et al.

Sciuro-hypnum (Hampe) Hampe

Scorpiurium Schimp.

Calliergonaceae (Kanda) Vanderp., Hedenäs,

C.J.Cox \& A.J.Shaw

Calliergon (Sull.) Kindb.

Conardia H.Rob.

Loeskypnum H.K.G.Paul

Straminergon Hedenäs

Warnstorfia Loeske

Scorpidiaceae Ignatov \& Ignatova

Hamatocaulis Hedenäs

Hygrohypnella Ignatov \& Ignatova

Sanionia Loeske

Scorpidium (Schimp.) Limpr.

Pylaisiaceae Schimp.

Breidleria Loeske

Callicladium H.A.Crum

Calliergonella Loeske

Gollania Broth.

Homomallium (Schimp.) Loeske
Pseudohygrohypnum Kanda

Ptilium De Not.

Pylaisia Bruch et al.

Stereodon (Brid.) Mitt.

Rhytidiaceae Broth.

Rhytidium (Sull.) Kindb.

Pseudoleskeellaceae Ignatov \& Ignatova Pseudoleskeella Kindb.

Leskeaceae Schimp.

*Claopodium (Lesq. \& James) Renauld \& Cardot

*Iwatsukiella W.R.Buck \& H.A.Crum

Leskea Hedw.

Lindbergia Kindb.

Mamillariella Laz.

* Miyabea Broth.

Pseudoleskeopsis Broth.

Thuidiaceae Schimp.

Abietinella Müll.Hal.

Actinothuidium (Besch.) Broth.

Boulaya Cardot

Bryochenea C.Gao \& G.C.Zhang

Bryonoguchia Z.Iwats. \& Inoue

Echinophyllum O'Brian

Haplocladium (Müll.Hal.) Müll.Hal.

Helodium Warnst.

Pelekium Mitt.

Rauiella Reimers

Thuidium Bruch et al.

Amblystegiaceae G.Roth

Amblystegium Bruch et al.

Anacamptodon Brid.

Campyliadelphus (Kindb.) R.S.Chopra

Campylidium (Kindb.) Ochyra

Campylium (Sull.) Mitt.

*Campylophyllum (Schimp.) M.Fleisch.

Cratoneuron (Sull.) Spruce

*Drepanium C.E.O.Jensen

Drepanocladus (Müll.Hal.) G.Roth

Hygroamblystegium Loeske

Hygrohypnum Lindb.

Leptodictyum (Schimp.) Warnst.

*Myrinia Schimp.

*Ochyraea Váňa

Palustriella Ochyra

*Podperaea Z.Iwats. \& Glime

Pseudocalliergon (Limpr.) Loeske

Sasaokaea Broth.

Serpoleskea (Limpr.) Loeske

*Tomentypnum Loeske 


\section{LIST OF TAXA ${ }^{1}$}

Abietinella Müll.Hal. [Thuidiaceae]

- abietina (Hedw.) M.Fleisch. (Thuidium abietinum (Hedw.) Bruch et al., Abietinella hystricosa (Mitt.) Broth., A. abietina var. hystricosa (Mitt.) Sakurai) - LTV [244], LAT [3], EST [237], BEL [355], UKR: C [66], M [66], K [66, 328], MLD [367], RUS-EUR: ARC [47, 127, 150], NW [207, 212, 364, 407], NE [207, 212, 415], KLN [309], C [207, 212], N-UR [150, 207, 212], S-UR [150, 207, 212], SE [207, 212], CAUC [55, 212, 231, 248], GEO [117], ARM [297], AZE [277], KAZ [156, 296, 356], TAD [296], KYR [296, 349, 351], RUS-AS: ARC-WSIB [136], ARC-ESIB [164, 212, 275], W-SIB [212, 265], S-SIB [69, 70, 212], E-SIB [69, 212, 275], ARC-YAK [47, 212, 239], YAK [212, 239], ARC-FE [40, 212], N-FE [94, 140], S-FE [76, 77, 114, 212]

Acaulon Müll.Hal. [Pottiaceae]

— muticum (Hedw.) Müll.Hal. - LTV [244], LAT [5], BEL [355], UKR: M [66], RUS-EUR: C [207, 345], TUR [295], TAD [295], KYR [349, 351], RUS-AS: ?W-SIB [341]

- triquetrum (Spruce) Müll.Hal. - UKR: M [66], RUS-EUR: S-UR [150, 341], SE [207, 212, 377], CAUC [421], GEO [117], AZE [277], TUR [296], TAD [295]

Actinothuidium (Besch.) Broth. [Thuidiaceae]

- hookeri (Mitt.) Broth. subsp. boreale Ignatov, Ignatova, Z.Iwats. \& B.C.Tan - RUS-AS: S-FE [210, 212, 221]

Aloina Kindb. [Pottiaceae]

— aloides (Koch ex Schultz) Kindb. - LTV [244], TAD [295]

- ambigua (Bruch et al.) Limpr. (A. ericaefolia Kindb.) - BEL [?355], UKR: C [66], M [66], MLD [367], AZE [277], TUR [267, 295, 296], TAD [294, 295], KYR [296, 349, 351] - \{1\}

- brevirostris (Hook. \& Grev.) Kindb. - LAT [4], UKR: M [66], RUS-EUR: NW [207], NE [207, 212], C [207, 212, 345], S-UR [150, 207], CAUC [250], TUR [296], TAD [295], RUS-AS: ARC-WSIB [136], ARC-ESIB [164, 212, 275], W-SIB [265], S-SIB [197, 212], E-SIB [212, 275], ARC-YAK [47, 239],YAK [239], ARC-FE [40, 212], N-FE [94]

— rigida (Hedw.) Limpr. - LTV [244], LAT [3], EST [237], BEL [?355], UKR: C [66], M [66], RUSEUR: NW [207], C [207, 345], S-UR [150, 207], CAUC [231, 248], GEO [117], AZE [277], TUR [267, 295], TAD [294, 295], KYR [296, 351], RUS-AS: S-SIB [69, 70, 212], E-SIB [69], YAK [239]

Amblyodon P.Beauv. [Meesiaceae]

- dealbatus (Hedw.) P.Beauv. - LTV [244], LAT [3], EST [237], BEL [355], UKR: M [66, 148], RUSEUR: NW [207, 273, 364, 407], NE [207, 212], KLN [309], C [207], N-UR [150], CAUC [55, 102, 212, 248], GEO [102, 117], ARM [297], KAZ [156, 393], TAD [267, 294, 296], KYR [296], RUSAS: S-SIB [69, 70, 197], YAK [212, 239], ARC-FE [40]

Amblystegiella see Serpoleskea, Platydictya

Amblystegium Bruch et al. [Amblystegiaceae], see also Hygroamblystegium humile, H. varium, etc., Serpoleskea subtilis, etc., Conardia compacta

- radicale (P.Beauv.) Bruch et al. (A. saxatile Schimp., Campylium radicale (P.Beauv.) Grout) - EST [237], BEL [355], UKR: C [66], M [66], K [66, 328], RUS-EUR: NW [291], NE [414], C [135, 212, 344], S-UR [150], RUS-AS: W-SIB [265], S-SIB [70, 212, 275], E-SIB [275], S-FE [76]

Notes: 1) References to annotations on some species are given in figure brackets.

2) Asterisk (*) at some family names means that the position of the genus in this family is not well proved.

3) Asterisk (*) at some record means indirect record: for example, old records of Eurhynchium striatum from Siberia, before the description of E. zetterstedtii (E. angustirete), obviously mean the latter species.

4) Questionmark (?) before the reference number in brackets means that the author(s) of reference express(es) certain doubt of the occurrence of the taxon in region; "?" before the region name however indicate the doubts of authors of the checklist; "?" before species name means that we doubt that this is a good species, but retain it due to unknown identity.

5) Few species were reported from Middle Asia and Caucasus without exact locality, so even the country can not be detected. In these cases they are cited: + MID-AS and + CAUCASUS.

6) Only synonyms that were widely used in recent literature are included in the list; expanded list of synonyms is given in a separate section after Annotations. 
- serpens (Hedw.) Bruch et al. - LTV [244], LAT [3], EST [237], BEL [355], UKR: C [66], M [66], K [66, 328], MLD [367], RUS-EUR: ARC [47, 150], NW [207, 212, 364, 407], NE [207, 212, 415], KLN [309], C [207, 212], N-UR [150, 207], S-UR [150, 207, 212], SE [207, 212, 404], CAUC [55, 208, 212, 231, 248], GEO [117], ARM [297], AZE [277], KAZ [160, 296, 356], TAD [26, 296], KYR [296], RUS-AS: ARC-WSIB [136], W-SIB [212, 265], S-SIB [69, 70, 212], E-SIB [69, 212, 275], ARC-YAK [47, 239], YAK [212, 239], ARC-FE [40], N-FE [94, 140], S-FE [76, 77, 109, 212]

- serpens var. juratzkanum (Schimp.) Rau \& Herv. - LTV [244], LAT [1], EST [237], BEL [355], UKR: C [66], M [66], K [66, 328], MLD [367], RUS-EUR: NW [63, 407], NE [207], KLN [309], C [207, 212], N-UR [150], S-UR [150, 207, 212], CAUC [55, 212, 248], GEO [117], ARM [297], AZE [277], KAZ [296, 356], UZB [267], TAD [294, 296], KYR [267, 296], RUS-AS: W-SIB [212, 265], S-SIB [69, 70, 212], E-SIB [212, 247], ARC-YAK [239], YAK [212, 239], ARC-FE [40], S-FE [76, 77, 212]

Amphidium Schimp. [Rhabdoweisiaceae]

- Iapponicum (Hedw.) Schimp. - UKR: C [66], RUS-EUR: ARC [47, 150], NW [212, 273, 364, 407], N-UR [150, 207, 212, 415], S-UR [150, 207, 212], CAUC [212, 231, 248], GEO [117], ARM [297], KAZ [156, 159], UZB [267], TAD [294, 295], KYR [296, 349, 351], RUS-AS: S-SIB [69, 70, 212], E-SIB [69, 212], ARC-YAK [47, 239], YAK [239], ARC-FE [40], N-FE [140], S-FE [76, 77, 114, 212]

- mougeotii (Bruch et al.) Schimp. - UKR: C [66], K [328], RUS-EUR: ARC [47, 127, 150], NW [212, 364, 407], N-UR [150, 207, 212, 415], S-UR [207], CAUC [55, 212, 231, 248], GEO [117], ARM [297], KAZ [156, 159], TAD [294, 295], KYR [296], RUS-AS: ARC-ESIB [39, 164], S-SIB [197, 212], E-SIB [163], ARC-FE [40, 212], N-FE [40, 94], S-FE [221]

Anacamptodon Brid. [Amblystegiaceae]

- kamchaticus Czernyadjeva - RUS-AS: N-FE [139, 140], S-FE [139]

— latidens (Besch.) Broth. - RUS-AS: S-SIB [69, 70, 212], E-SIB [69, 212], S-FE [76, 77, 109, 212]

— splachnoides (Froel. ex Brid.) Brid. - UKR: C [66], GEO [117], RUS-AS: ?S-SIB [22], ?S-FE [22] — $\{2\}$ Anacolia Schimp. [Bartramiaceae]

- webbii (Mont.) Schimp. - TAD [19, 296]

Andreaea Hedw. [Andreaeaceae]

- alpestris (Thed.) Bruch et al. (A. fauriei Besch.) - UKR: C [66], RUS-EUR: ARC [47], NW [364, 407], CAUC [55, 212], RUS-AS: S-SIB [197, 212], E-SIB [212, 247], ARC-YAK [47, 239], ARC-FE [40], N-FE [140], S-FE [77, 109]

- blyttii Bruch et al. - RUS-EUR: NW [83, 87, 273], RUS-AS: YAK [239], ARC-FE [40, 212]

- crassinervia Bruch - RUS-EUR: NW [262, 364, 407]

- heinemannii Hampe \& Müll.Hal. (A. planinervis Lindb. ex G.Roth) - RUS-EUR: CAUC [248], GEO [102], RUS-AS: S-SIB [197, 212] $-\{3\}$

- nivalis Hook. - RUS-EUR: ARC [47], NW [84, 87], N-UR [150, 207, 212], CAUC [55, 212, 231], RUS-AS: N-FE [95, 140], S-FE [67]

- obovata Thed. - RUS-EUR: ARC [47, 150], NW [364, 407], N-UR [150], RUS-AS: S-SIB [69, 70, 212], E-SIB [212, 247], YAK [239], ARC-FE [40]

- rothii F.Weber \& D.Mohr - RUS-EUR: ARC [127, 150], NW [262], N-UR [166], ?S-UR [150, 207], GEO [117] - $\{3\}$

- rupestris Hedw. - LAT [3], EST [237], BEL [355], UKR: C [66], RUS-EUR: ARC [47, 127, 150], NW [207, 212, 364, 407], NE [207, 212, 415], N-UR [150, 207, 212], S-UR [150, 207, 212], CAUC [55, 212, 231, 248], GEO [117], ARM [297], AZE [277], TAD [295], RUS-AS: ARC-WSIB [136], ARC-ESIB [164, 212], S-SIB [69, 70, 212], E-SIB [69, 212, 275], ARC-YAK [47, 212, 239], YAK [239], ARC-FE [40, 212], N-FE [94, 140], S-FE [76, 212]

- rupestris var. papillosa (Lindb.) Podp. - RUS-EUR: ARC [47, 150], S-UR [150, 212], RUS-AS: ARC-WSIB [136], ARC-ESIB [164, 212], S-SIB [197, 212], E-SIB [388], ARC-YAK [47, 212, 239], YAK [239], ARC-FE [40, 212], N-FE [140]

- rupestris var. sparsifolium (J.E.Zetterst.) Sharp - RUS-AS: ARC-FE [40], N-FE [40]

Anisothecium see Dicranella spp., Dichodontium paluste

Anoectangium Schwägr. [Pottiaceae], see also Molendoa

- aestivum (Hedw.) Mitt. - UKR: C [66], RUS-EUR: NW [85, 212, 364], CAUC [55, 102, 212, 231], 
TUR [11, 296], RUS-AS: E-SIB [162], ARC-YAK [239]

- handelii Schiffn. - UKR: K [66, 328], RUS-AS: S-SIB [71], TUR [8, 368], TAD [294, 295]

- thomsonii Mitt. (A. amurense Broth., A. contortum Broth.) - RUS-AS: E-SIB [74, 212], YAK [239], S-FE [76, 212, 221]

Anomobryum Schimp. [Bryaceae]

— julaceum (Schrad. ex P.Gaertn., B.Mey. \& Scherb.) Schimp. - UKR: C [66], RUS-EUR: NW [364], CAUC [212, 231, 248], GEO [117], ARM [297], AZE [277], RUS-AS: S-SIB [69, 70, 212], E-SIB [69, 212], ARC-YAK [47, 239], YAK [239], ARC-FE [40], N-FE [40, 140], S-FE [77, 212]

- julaceum var. concinnatum (Spruce) J.E.Zetterst. (A. concinnatum (Spruce) Lindb.) - KAZ [296, 356], TAD [294, 296], RUS-AS: ARC-FE [40], S-FE [76] $-\{4\}$

Anomodon Hook. \& Taylor [Anomodontaceae], see also Claopodium rostratum

— abbreviatus Mitt. - RUS-AS: S-FE [116]

- attenuatus (Hedw.) Huebener - LTV [244], LAT [3], EST [237], BEL [355], UKR: C [66], M [66], K [66, 328], MLD [367], RUS-EUR: NW [207, 407], KLN [309], C [207, 212], N-UR [150, 207, 212], S-UR [150, 207, 212], SE [207], CAUC [55, 208, 212, 231, 248], GEO [117], ARM [297], AZE [277], TAD [296], RUS-AS: S-SIB [69, 70, 212], E-SIB [212, 247]

— giraldii Müll.Hal. - RUS-AS: S-FE [76, 77, 109, 212]

- longifolius (Brid.) Hartm. - LTV [244], LAT [3], EST [237], BEL [355], UKR: C [66], M [66], K [66, 328], MLD [367], RUS-EUR: NW [207, 212, 364, 407], NE [207, 212, 415], KLN [309], C [207, 212], N-UR [150, 207, 212, 415], S-UR [150, 207, 212], SE [207], CAUC [55, 208, 212, 232, 248], GEO [117], ARM [297], AZE [277], RUS-AS: S-SIB [69, 70, 212], N-FE [140], S-FE [76, 77, 109, 212]

- minor (Hedw.) Fürnr. (A. minor subsp. integerrimus (Mitt.) Z.Iwats.) - RUS-AS: S-SIB [69, 70, 212], E-SIB [74, 212], YAK [212, 239], S-FE [76, 77, 109, 212] - $\{5\}$

— rugelii (Müll.Hal.) Keissl. - EST [237], UKR: C [66], M [66], RUS-EUR: NW [207], C [207], S-UR [150, 207, 212], CAUC [55, 212, 231, 248], GEO [117], ARM [297], AZE [277], RUS-AS: S-SIB [69, 197, 212], E-SIB [212, 247], S-FE [76, 77, 109, 212]

- solovjovii Laz. - RUS-AS: S-FE [76, 212]

— thraustus Müll.Hal. - RUS-AS: S-SIB [69, 70], E-SIB [69, 212], S-FE [76, 77, 109, 212]

— viticulosus (Hedw.) Hook. \& Taylor - LTV [244], LAT [3], EST [237], BEL [355], UKR: C [66], M [66], K [328], MLD [367], RUS-EUR: NW [207, 212, 364, 407], NE [53, 207, 212, 415], KLN [309], C [207, 212], N-UR [150, 207, 212, 415], S-UR [150, 207, 212], CAUC [55, 208, 212, 231, 248], GEO [117], ARM [297], AZE [277], KAZ [156, 159, 296], KYR [296], RUS-AS: S-SIB [70, 212], N-FE [?140]

Antitrichia Brid. [Antitrichiaceae]

- curtipendula (Hedw.) Brid. - LTV [244], LAT [3], EST [237], BEL [355], UKR: C [66], M [66], K [66, 328], RUS-EUR: NW [203, 207, 407], KLN [309], CAUC [55, 203, 208, 212, 231], GEO [117], ARM [297]

Aongstroemia Bruch et al. [Dicranaceae]

— julacea (Hook.) Mitt. - RUS-AS: S-SIB [70]

- longipes (Sommerf.) Bruch et al. - RUS-EUR: NW [207, 251], RUS-AS: ARC-WSIB [136], ARCESIB [164, 212], E-SIB [163], ARC-FE [40, 212], N-FE [40, 140]

— orientalis Mitt. - RUS-AS: S-SIB [69]

Aplodon R.Br. [Splachnaceae]

— wormskioldii (Hornem.) R.Br. - EST [237], RUS-EUR: ARC [47, 150], NW [212, 346, 364], RUSAS: ARC-WSIB [136], ARC-ESIB [164, 212, 275], E-SIB [126, 212, 275], ARC-YAK [47, 239], ARC-FE [40, 212], N-FE [40, 94]

Archidium Brid. [Archidiaceae]

— alternifolium (Hedw.) Mitt. - LTV [244], RUS-EUR: NW [207, 262], RUS-AS: S-FE [24, 76]

Arctoa Bruch et al. [Rhabdoweisiaceae]

- anderssonii Wich. - RUS-AS: ARC-ESIB [39, 47, 50], N-FE [141]

- fulvella (Dicks.) Bruch et al. - UKR: C [66], RUS-EUR: ARC [47, 150], NW [212, 273, 364, 407], NUR [150], RUS-AS: ARC-ESIB [96, 212], S-SIB [69], E-SIB [69, 212], ARC-YAK [239], YAK [239], ARC-FE [40], N-FE [94, 140], S-FE [212, 221] 
Astomum see Weissia

Atractylocarpus alpinus (Schimp. ex Milde) Lindb. - excluded $-\{6\}$

Atrichum P.Beauv. [Polytrichaceae]

— angustatum (Brid.) Bruch et al. - LTV [244], LAT [3], EST [237], BEL [355], UKR: C [66], M [66], RUS-EUR: NW [87, 207, 262], C [207, 212], S-UR [207], CAUC [55, 212, 248], GEO [117]

- flavisetum Mitt. (A. haussknechtii Jur. \& Milde, A. undulatum var. gracilisetum Besch.) - LAT [1], EST [237], BEL [355], UKR: C [66], M [66], K [328], RUS-EUR: NW [207, 262, 407], NE [207, 212], C [207, 212, 345], N-UR [90, 150, 207, 212], S-UR [150, 207, 212], CAUC [55, 212, 231, 248], GEO [117], ARM [297], AZE [277], KAZ [296], RUS-AS: W-SIB [265], S-SIB [69, 70, 212, 219], E-SIB [212, 247], S-FE [76, 77, 109, 212]

- rhystophyllum (Müll.Hal.) Paris - RUS-AS: S-FE [76] - $\{7\}$

- tenellum (Röhl.) Bruch et al. - LTV [244], LAT [3], EST [237], BEL [355], UKR: C [66], M [66], RUS-EUR: NW [207, 212, 364, 407], NE [207, 212, 415], KLN [309], C [207, 212, 345], N-UR [150, 207, 212], S-UR [150, 207, 212], RUS-AS: W-SIB [212, 265], S-SIB [69, 70, 212, 219], E-SIB [212, 247], ?ARC-YAK [47, 239], ?N-FE [140], ?S-FE [77, 212] - \{8\}

- undulatum (Hedw.) P.Beauv. - LTV [244], LAT [3], EST [237], BEL [355], UKR: C [66], M [66], K [66, 328], MLD [367], RUS-EUR: ARC [150], NW [207, 212, 364, 407], NE [207], KLN [309], C [207, 212], N-UR [150, 207, 212, 415], S-UR [150, 207, 212], SE [207, 212], CAUC [55, 208, 212 , 231, 248], GEO [117], ARM [297], AZE [277], KAZ [296], RUS-AS: W-SIB [265], S-SIB [70, 212, 219], S-FE [76, 109, 212]

Aulacomnium Schwägr. [Aulacomniaceae]

- acuminatum (Lindb. \& Arnell) Kindb. - RUS-AS: ARC-WSIB [136], ARC-ESIB [92, 212], S-SIB [69, 70, 212], E-SIB [212, 275], ARC-YAK [47, 212, 239], YAK [212, 239], ARC-FE [40, 212], N-FE [94]

— androgynum (Hedw.) Schwägr. - LTV [244], LAT [3], EST [237], BEL [355], UKR: C [66], M [66], K [66, 328], RUS-EUR: NW [207, 212, 262], KLN [309], SE [207], GEO [117] — \{9\}

- heterostichum (Hedw.) Bruch et al. - RUS-AS: S-FE [76, 77, 109, 212]

- palustre (Hedw.) Schwägr. (A. palustre var. imbricatum Bruch et al.) - LTV [244], LAT [3], EST [237], BEL [355], UKR: C [66], M [66], RUS-EUR: ARC [47, 127, 150], NW [207, 212, 364, 407], NE [207, 212, 415], KLN [309], C [207, 212], N-UR [150, 207, 212], S-UR [150, 207, 212], SE [207, 212], CAUC [55, 212, 231, 248], GEO [117], ARM [297], AZE [277], KAZ [23, 156, 296, 356], TAD [26, 296], KYR [296, 351], RUS-AS: ARC-WSIB [136], ARC-ESIB [164, 212], W-SIB [212, 265], S-SIB [69, 70, 212], E-SIB [69, 212], ARC-YAK [47, 212, 239], YAK [212, 239], ARC-FE [40, 212], N-FE [94, 140], S-FE [76, 77, 109, 212]

- turgidum (Wahlenb.) Schwägr. - RUS-EUR: ARC [47, 127, 150], NW [212, 291, 364, 407], NE [207, 212, 415], N-UR [150, 207, 212], S-UR [150, 207, 212], ARM [297], KAZ [296, 356], TAD [296], RUS-AS: ARC-WSIB [136], ARC-ESIB [164, 212], W-SIB [265], S-SIB [69, 70, 212], E-SIB [69, 212, 275], ARC-YAK [47, 212, 239], YAK [212, 239], ARC-FE [40, 212], N-FE [94, 140], S-FE [109, 212, 221]

Barbula Hedw. [Pottiaceae], see also Didymodon, Bryoerythrophyllum, Pseudocrossidium

— amplexifolia (Mitt.) A.Jaeger - RUS-AS: S-SIB [212, 222], YAK [239]

- convoluta Hedw. (Streblotrichum convolutum (Hedw.) P.Beauv.) - LTV [244], LAT [3], EST [237], BEL [355], UKR: C [66], M [66], K [66, 328], RUS-EUR: NW [207, 273, 364, 407], NE [207, 212, 415], KLN [309], C [207, 212], N-UR [90, 150, 207, 212], S-UR [150, 207], SE [207], CAUC [55, 212, 232, 248], GEO [117], ARM [297], AZE [277], KAZ [157, 296, 356], TUR [296], TAD [294, 295], KYR [267, 296], RUS-AS: ARC-ESIB [164], W-SIB [265], S-SIB [69, 70, 212], E-SIB [163, 275, 388], YAK [239], N-FE [40, 140], S-FE [76, 212]

- crocea (Brid.) F.Weber \& D.Mohr - UKR: C [66, 413], RUS-EUR: CAUC [55, 212, 248]

- enderesii Garov. - UKR: M [66], UZB [267], KYR [296]

- indica (Hook.) Spreng. - UZB [384]

- jacutica Ignatova - RUS-AS: E-SIB [162], YAK [223, 239]

— unguiculata Hedw. - LTV [244], LAT [3], EST [237], BEL [355], UKR: C [66], M [66], K [66, 328], MLD [367], RUS-EUR: NW [207, 212, 364, 407], NE [207, 212, 415], KLN [309], C [207, 212], NUR [150, 207, 212], S-UR [150, 207, 212], SE [207, 212], CAUC [55, 208, 212, 231, 248], GEO 
[117], ARM [297], AZE [277], KAZ [157], TUR [296], UZB [295, 296], TAD [294, 295], KYR [296], RUS-AS: ARC-ESIB [275], W-SIB [265], S-SIB [69, 70, 212], E-SIB [163, 275], YAK [239], N-FE [140], S-FE [76, 221]

Bardunovia Ignatov \& Ochyra [Plagiotheciaceae]

- baicalensis Ignatov \& Ochyra (Platydictya baicalensis (Ignatov \& Ochyra) Hedenäs) - RUS-AS: ESIB [218], YAK [239] $-\{10\}$

Bartramia Hedw. [Bartramiaceae]

- halleriana Hedw. - UKR: C [66], K [328], RUS-EUR: NW [364, 407], CAUC [55, 212, 231], GEO [117], ARM [297], AZE [277], TAD [296]

- ithyphylla Brid. (B. deciduaefolia Broth. \& Yasuda) - LTV [244], LAT [3], EST [237], UKR: C [66], M [66], RUS-EUR: ARC [47, 127, 150], NW [212, 273, 364, 407], NE [53, 207, 415], KLN [309], NUR [150, 207, 212], S-UR [150, 207, 212], CAUC [55, 212, 231, 248], GEO [117], ARM [297], AZE [277], KAZ [160, 296, 356], TAD [296], KYR [296], RUS-AS: ARC-WSIB [136], ARC-ESIB [164, 212, 275], W-SIB [147, 212], S-SIB [69, 70, 212], E-SIB [69, 212, 275], ARC-YAK [47, 212, 239], YAK [239], ARC-FE [40, 212], N-FE [94, 140], S-FE [212, 221]

- pomiformis Hedw. - LTV [244], LAT [3], EST [237], BEL [355], UKR: C [66], M [66], K [66, 328], RUS-EUR: ARC [47, 127, 150], NW [207, 212, 364, 407], NE [53, 207, 212], KLN [309], C [207], NUR [150, 207, 212, 415], S-UR [150, 207, 212], CAUC [55, 212, 248], GEO [117], AZE [277], KAZ [296, 356], TAD [296], RUS-AS: ARC-ESIB [164, 212], S-SIB [69, 70, 212], E-SIB [69, 70, 212, 275], ARC-YAK [47, 212, 239], YAK [212, 239], ARC-FE [40, 212], N-FE [94, 140], S-FE [76, 77, 109, 212]

- stricta Brid. - AZE [22]

- subulata Bruch et al. - RUS-EUR: ARC [47, 127, 150], RUS-AS: S-SIB [69, 70, 212], E-SIB [69], YAK [239], ARC-FE [40]

Bartramiopsis Kindb. [Polytrichaceae]

— lescurii (James) Kindb. - RUS-AS: N-FE [94, 140], S-FE [76, 77, 109, 212]

Blindia Bruch et al. [Seligeriaceae]

- acuta (Hedw.) Bruch et al. (B. seligerioides Lindb. ex Broth.) - UKR: C [66], RUS-EUR: ARC [47, 127, 150], NW [212, 273, 364, 407], NE [207, 415], N-UR [150, 207, 212], CAUC [55, 212, 231, 248], GEO [117], AZE [277], KAZ [104], RUS-AS: ARC-ESIB [39, 47, 50], S-SIB [69, 70, 212], ESIB [74, 212, 247], YAK [239], ARC-FE [40, 212], N-FE [40, 94], S-FE [212, 221]

Boulaya Cardot [Thuidiaceae]

— mittenii (Broth.) Cardot - RUS-AS: S-FE [76, 77, 109, 212]

Brachydontium Fürnr. [Seligeriaceae]

- olympicum (E.Britton) McIntosh \& Spense (Grimmia olympica E.Britton) - RUS-AS: S-FE [299]

— trichodes (F.Weber) Milde - UKR: C [66], RUS-EUR: N-UR [150, 415] + CAUCASUS [341]

Brachymenium Schwägr. [Bryaceae]

— exiloides Bard. \& Czerd. - RUS-AS: S-FE [76]

- nepalense Hook. - RUS-AS: S-FE [76, 212]

Brachytheciastrum Ignatov \& Huttunen [Brachytheciaceae]

- collinum (Schleich. ex Müll.Hal.) Ignatov \& Huttunen (Brachythecium collinum (Schleich. ex Müll.Hal.) Bruch et al., B. fendleri auct.) - RUS-EUR: S-UR [52], SE [207, 212], CAUC [212, 231, 248], GEO [117], ARM [297], AZE [277], KAZ [156, 296, 356, 393], TUR [296], UZB [267], TAD [267, 294, 296], KYR [296, 351], RUS-AS: S-SIB [198, 212], ARC-YAK [47, 239], ARC-FE [40], N-FE [140] — \{11\}

- falcatulum (Broth.) Ignatov \& Huttunen - KAZ [198], KYR [198], RUS-AS: S-SIB [198]

- olympicum (Jur.) Vanderp., Ignatov, Huttunen \& Goffinet (Brachythecium olympicum Jur.) - RUSEUR: SE [366], CAUC [232]

- trachypodium (Brid.) Ignatov \& Huttunen (Brachythecium trachypodium (Brid.) Bruch et al.) - RUSEUR: NW [364], S-UR [91], GEO [117], KAZ [348], UZB [267], TAD [294, 296], KYR [267, 296, 351], RUS-AS: ARC-ESIB [93], S-SIB [198], E-SIB [163], ARC-YAK [47, 239], YAK [239], ARCFE [40], N-FE [94] - $\{12\}$

— velutinum (Hedw.) Ignatov \& Huttunen (Brachythecium velutinum (Hedw.) Bruch et al.) - LTV [244], LAT [3], EST [237], BEL [355], UKR: C [66], M [66], K [66], MLD [367], RUS-EUR: ARC [47, 
150], NW [207, 212, 364, 407], NE [207, 212, 415], KLN [309], C [207, 212], N-UR [150, 207, 212], S-UR [150, 207, 212], SE [207, 212], CAUC [55, 208, 212, 231, 248], GEO [117], ARM [297], AZE [277], KAZ [159], TUR [296], TAD [296], KYR [296, 349, 351], RUS-AS: ARC-ESIB [164, 212], W-SIB [212, 265], S-SIB [69, 70, 198, 212, 275], E-SIB [212, 247], YAK [212, 239], ARC-FE [40, 212], N-FE [140], S-FE [76, 109, 212] - $\{13\}$

Brachythecium Bruch et al. [Brachytheciaceae], see also Brachytheciastrum collinum, B. velutinum, etc., Sciuro-hypnum oedipodium, S. plumosum, S. populeum, S. reflexum, etc. $-\{14\}$

— albicans (Hedw.) Bruch et al. - LTV [244], LAT [3], EST [237], BEL [355], UKR: C [66], M [66], K [66, 328], MLD [367], RUS-EUR: ARC [47, 150], NW [207, 212, 364, 407], NE [207, 212, 415], KLN [309], C [207, 212], N-UR [150, 207, 212], S-UR [150, 207, 212], SE [207, 212], CAUC [55, 212, 231], GEO [117], ARM [297], AZE [277], KAZ [159, 296, 356], TUR [296], TAD [294, 296], KYR [296, 351], RUS-AS: ARC-WSIB [136], W-SIB [265], S-SIB [69, 70, 212], E-SIB [212, 386], ARC-YAK [47, 239], YAK [239], ARC-FE [40], N-FE [140], S-FE [67]

- auriculatum A.Jaeger - RUS-AS: S-FE [77, 109, 212, 268] - $\{15\}$

- buchananii (Hook.) A.Jaeger (B. abakanense Kaal.) - UZB [206], RUS-AS: S-SIB [69, 70, 198, 212], YAK [239], N-FE [140], S-FE [114, 212, 221]

- campestre (Müll.Hal.) Bruch et al. - LTV [244], LAT [3], EST [237], BEL [355], UKR: C [66], M [66], K [328], MLD [367], RUS-EUR: ARC [47, 150, 415], NW [207, 212, 364, 407], NE [207, 415], KLN [309], C [207, 212], N-UR [150, 207, 415], S-UR [150, 207, 212], CAUC [212, 231, 248], GEO [117], ARM [297], AZE [277], KAZ [159, 296, 356], TUR [296], TAD [293, 296], KYR [267, 296, 351], RUS-AS: W-SIB [265], S-SIB [70], E-SIB [163], YAK [212, 258], ARC-FE [40], N-FE [40, 140], S-FE [76, 109, 212] - $\{16\}$

- capillaceum (F.Weber \& D.Mohr) Giacom. (B. rotaeanum De Not., B. salebrosum subsp. rotaeanum (De Not.) J.J.Amann, B. salebrosum var. capillaceum (F.Weber \& D.Mohr) Lorentz) - ?BEL [355], MLD [367], RUS-EUR: NW [289], NE [207, 212], C [207, 212], N-UR [90, 150, 207, 212], S-UR [150, 207, 212], SE [207, 212], CAUC [212, 231, 248], GEO [117], AZE [277], RUS-AS: W-SIB [265], S-SIB [198, 212, 265], YAK [239], N-FE [140], S-FE [114, 212, 221]

- ?caucasicum Thér. - GEO [382] - $\{17\}$

- cirrosum (Schwägr.) Schimp. (Cirriphyllum cirrosum (Schwägr.) Grout) - UKR: C [66], RUS-EUR: ARC [47, 150], NW [207, 364, 407], NE [207, 415], N-UR [150, 207, 212], S-UR [150], CAUC [55, 212, 231, 248], ARM [297], AZE [277], KAZ [156, 159, 296, 356], TAD [296], KYR [296], RUSAS: ARC-WSIB [136], ARC-ESIB [164, 212], S-SIB [69, 70, 198, 212], E-SIB [69, 212], ARC-YAK [47, 212, 239], YAK [212, 239], ARC-FE [40, 212], N-FE [94, 140], S-FE [212, 221]

- complanatum Broth. - RUS-AS: S-SIB [198, 212], E-SIB [388], S-FE [212, 215, 221]

- coruscum I.Hagen - RUS-EUR: NW [364], RUS-AS: ARC-WSIB [128], ARC-ESIB [39], ARC-FE $[40,212], \mathrm{N}-\mathrm{FE}[40]-\{18\}$

- erythrorrhizon Bruch et al. - LAT [3], EST [237], RUS-EUR: ARC [47, 127, 150], NW [207, 212, 364, 407], NE [207, 415], N-UR [150, 207], S-UR [150], CAUC [212, 231], TAD [296], RUS-AS: ARC-WSIB [136], S-SIB [70], ARC-YAK [47], ARC-FE [40], N-FE [140] $-\{19\}$

- erythrorrhizon subsp. asiaticum Ignatov - RUS-EUR: NE [207, 212], C [207, 212], S-UR [207, 212], RUS-AS: ARC-WSIB [136], W-SIB [212, 265], S-SIB [198, 212, 265], E-SIB [74, 212, 247], YAK $[212,239]$, S-FE $[212,221]-\{19\}$

— frigidum (Müll.Hal.) Besch. (Brachythecium asperrimum (Mitt. ex Müll. Hal.) Sull.) - KAZ [356] — $\{19 \mathrm{a}\}$

- garovaglioides Müll.Hal. (B. wichurae (Broth.) Paris - excluded - $\{20\}$

- geheebii Milde (Homalothecium geheebii (Milde) Wigh) - UKR: C [66], RUS-EUR: S-UR [150, 207, 212], CAUC [231, 248], GEO [117], ARM [297], TUR [9, 296]

- glareosum (Bruch ex Spruce) Bruch et al. - LTV [244], LAT [3], EST [237], BEL [355], UKR: C [66], M [66], K [66, 328], MLD [367], RUS-EUR: NW [207, 407], NE [207, 212, 415], KLN [309], C [207, 212], N-UR [207, 212], S-UR [91, 207, 212], SE [207], CAUC [55, 212, 231, 248], GEO [117], ARM [297], AZE [277], KAZ [156, 159, 296], KYR [267]

- kuroishicum Besch. - RUS-AS: S-FE [76, 77, 109, 212] 
— ?laetum (Brid.) Bruch et al. (B. oxycladon auct. non (Brid.) A.Jaeger) - ?UKR: C [66], ?RUS-EUR: SUR [150], ?AZE [277]

- mildeanum (Schimp.) Schimp. - LTV [244], LAT [3], EST [237], BEL [355], UKR: C [66], M [66], K [66, 328], MLD [367], RUS-EUR: ARC [47, 127, 150], NW [207, 212, 364, 407], NE [207, 415], KLN [309], C [207, 212], N-UR [150, 207, 212], S-UR [150, 207, 212], SE [207, 212], CAUC [55, 208, 212, 231, 248], GEO [117], AZE [277], KAZ [156, 296, 356], TAD [26, 296], KYR [296], RUS-AS: ARC-WSIB [136], ARC-ESIB [164, 212], W-SIB [212, 265], S-SIB [69, 70, 198, 212], ESIB [69, 212, 275], ARC-YAK [47, 212, 239], YAK [212, 239], ARC-FE [40, 212], N-FE [94, 140], S$\mathrm{FE}[76,212,221]$

- rivulare Bruch et al. - LTV [244], LAT [3], EST [237], BEL [355], UKR: C [66], M [66], K [66, 328], MLD [367], RUS-EUR: ARC [47, 150], NW [207, 212, 364, 407], NE [207, 212, 415], KLN [309], C [207, 212], N-UR [150, 207, 212], S-UR [150, 207, 212], SE [207], CAUC [55, 208, 212, 231, 248], GEO [117], ARM [297], AZE [277], KAZ [156, 159, 296, 356], UZB [267], TAD [294, 296], KYR [267, 296, 351], RUS-AS: W-SIB [265], S-SIB [69, 70, 198, 212], E-SIB [212, 275], YAK [239], N-FE [94, 140], S-FE [76, 77, 109, 212]

- rutabulum (Hedw.) Bruch et al. - LTV [244], LAT [3], EST [237], BEL [355], UKR: C [66], M [66], K [66, 328], MLD [367], RUS-EUR: NW [207, 212, 364, 407], NE [207, 212, 415], KLN [309], C [207, 212], N-UR [150, 207, 212], S-UR [150, 207, 212], SE [207, 212], CAUC [55, 208, 212, 231, 248], GEO [117], ARM [297], AZE [277], TAD [294, 296], KYR [296], RUS-AS: W-SIB [265], SSIB [197, 198, 212], E-SIB [275], S-FE [?76, 109, 212, 268, 340]

- salebrosum (F.Weber \& D.Mohr) Bruch et al. - LTV [244], LAT [3], EST [237], BEL [355], UKR: C [66], M [66], K [66, 328], MLD [367], RUS-EUR: ARC [47, 127, 150], NW [207, 212, 364, 407], NE [207, 212, 415], KLN [309], C [207, 212], N-UR [150, 207, 212], S-UR [150, 207, 212], SE [207, 212], CAUC [55, 208, 212, 231, 248], GEO [117], ARM [297], AZE [277], KAZ [156, 296, 356], TAD [294, 296], KYR [296, 351], RUS-AS: ARC-WSIB [136], ARC-ESIB [47], W-SIB [212, 265], S-SIB [69, 70, 198, 212], E-SIB [69, 212], ARC-YAK [47, 239], YAK [212, 239], ARC-FE [40, 212], N-FE [94, 140], S-FE [76, 77, 109, 212]

- tommasinii (Sendtn. ex Boulay) Ignatov \& Huttunen (Cirriphyllum tenuinerve (Lindb.) Wijk \& Margad., C. tommasinii (Sendtn. ex Boulay) Grout) - EST [237], UKR: C [66], M [66], K [66, 328], RUSEUR: NW [407], N-UR [90], GEO [117], AZE [277] — \{21\}

- turgidum (Hartm.) Kindb. - LAT [3], EST [237], RUS-EUR: ARC [47, 127, 150], NW [212, 273, 364, 407], NE [207, 415], N-UR [150, 207, 212], CAUC [231], KAZ [159, 296, 356], TAD [294, 296], KYR [296], RUS-AS: ARC-WSIB [136], ARC-ESIB [47, 164, 212], W-SIB [265], S-SIB [69, 70, 198, 212], E-SIB [126, 212], ARC-YAK [47, 212, 239], YAK [212, 239], ARC-FE [40, 212], N-FE [40, 140]

- udum I.Hagen - RUS-EUR: ARC [414], NW [85, 212, 364, 287], NE [414], RUS-AS: ARC-WSIB [136], ARC-ESIB [47], S-SIB [69, 70], E-SIB [163], ARC-YAK [47], YAK [239], ARC-FE [40, 212], N-FE [140]

Breidleria Loeske [Pylaisiaceae], see also Calliergonella

— pratensis (W.D.J.Koch ex Spruce) Loeske (Hypnum pratense W.D.J.Koch ex Spruce) - LTV [244], LAT [3], EST [237], BEL [355], UKR: C [66], M [66], RUS-EUR: ARC [47, 150], NW [207, 212, 364, 407], NE [207, 212, 415], KLN [309], C [207, 212], N-UR [150, 207, 415], S-UR [150, 207], RUS-AS: ARC-WSIB [136], ARC-ESIB [164], W-SIB [265], S-SIB [197, 200, 212], E-SIB [212, 386], YAK [212, 239], ARC-FE [40], N-FE [40, 140], S-FE [76, 109, 212]

Brothera Müll.Hal. [Leucobryaceae]

— leana (Sull.) Müll.Hal. - RUS-AS: S-SIB [69, 70, 212], S-FE [76, 212, 221]

Brotherella Loeske ex M.Fleisch. [Pylaisiadelphaceae], see also Pylaisiadelpha

— henonii (Duby) M.Fleisch. - RUS-AS: S-FE [77]

Bryhnia Kaurin [Brachytheciaceae]

- hultenii E.B.Bartram - RUS-AS: N-FE [40, 140], S-FE [109, 212, 221]

- novae-angliae (Sull. \& Lesq.) Grout (?B. brachycladula Cardot, ?B. noesica (Besch.) Broth.) - RUSAS: S-SIB [69, 212], N-FE [140], S-FE [77, 109, 212, 221]

- scabrida (Lindb.) Kaurin - RUS-EUR: NE [207], C [207], S-UR [207], RUS-AS: W-SIB [335], SSIB [198, 205, 207], YAK [239] 
Bryobrittonia R.S.Williams [Encalyptaceae]

- longipes (Mitt.) D.G.Horton - RUS-EUR: ARC [150, 415], NE [17], N-UR [150, 415], KAZ [296, 356], RUS-AS: ARC-ESIB [164, 193, 212], S-SIB [69, 70, 193, 212], E-SIB [193, 212], ARC-YAK [47, 212, 239], YAK [193, 212, 239], ARC-FE [40, 193, 212]

Bryochenea C.Gao \& G.C.Zhang [Thuidiaceae], see also Echinophyllum sachalinense

- vestitissima (Besch.) Touw (Thuidium vestitissimum Besch.) - RUS-AS: S-FE [76, 212]

Bryoerythrophyllum P.C.Chen [Pottiaceae]

- alpigenum (Venturi) P.C.Chen (B. recurvirostrum var. dentatum (Schimp.) H.A.Crum, Steere \& L.E.Anderson) - UKR: C [66], TAD [295], RUS-AS: S-SIB [197, 212], YAK [239], ARC-FE [40]

- ferruginascens (Stirt.) Giacom. - RUS-EUR: ARC [47], CAUC [212, 231, 248], RUS-AS: ARCESIB [164, 212], S-SIB [212, 227], E-SIB [163], ARC-YAK [227, 239], YAK [227, 239], ARC-FE [40, 212], N-FE [40], S-FE [212, 221, 227]

- inaequalifolium (Taylor) R.H.Zander - RUS-AS: S-SIB [196, 212]

- recurvirostrum (Hedw.) P.C.Chen - LTV [244], LAT [3], EST [237], BEL [355], UKR: C [66], M [66], K [66, 328], MLD [367], RUS-EUR: ARC [47, 127, 150], NW [207, 212, 364, 407], NE [207, 212, 415], KLN [309], C [207, 212], N-UR [150, 207, 212], S-UR [150, 207, 212], SE [207, 212], CAUC [55, 212, 231, 248], GEO [117], ARM [297], AZE [277], KAZ [156, 296, 356], TUR [296], UZB [296], TAD [294, 295], KYR [267, 296, 351], RUS-AS: ARC-WSIB [136], ARC-ESIB [164, 212, 275], W-SIB [212, 265], S-SIB [69, 70, 212], E-SIB [69, 212, 275], ARC-YAK [47, 212, 239], YAK [212, 239], ARC-FE [40, 212], N-FE [94, 140], S-FE [76, 77, 212]

- recurvirostrum var. brevifolium (Lindb. \& Arnell) Podp. - RUS-AS: ARC-FE [40]

- rotundatum (Lindb. \& Arnell) P.C.Chen - TAD [295], RUS-AS: ARC-ESIB [275], E-SIB [163]

- rubrum (Jur. ex Geh.) P.C.Chen - RUS-EUR: CAUC [102], RUS-AS: ARC-ESIB [39], ARC-FE [40] Bryohaplocladium see Haplocladium

Bryonoguchia Z.Iwats. \& Inoue [Thuidiaceae]

— molkenboeri (Sande Lac.) Z.Iwats. \& Inoue (Thuidium molkenboeri Sande Lac.) - RUS-AS: S-FE [76, 109, 212]

Bryoxiphium Mitt. [Bryoxiphiaceae]

- norvegicum (Brid.) Mitt. - RUS-AS: YAK [239], ARC-FE [40]

- norvegicum var. japonicum (Berggr.) A.Löve \& D.Löve (B. savatieri (Husn.) Mitt.) - RUS-AS: N-FE $[110,140]$, S-FE [76, 77, 212, 311, 358]

Bryum Hedw. [Bryaceae] - $\{22\}\{23\}$

- alexandri H.Philib. - KAZ [296] - $\{23\}$

- algovicum Sendtn. ex Müll.Hal. (B. pendulum (Hornsch.) Schimp., B. jailae Sapjegin, B. algovicum fo. jailae (Sapjegin) L.I.Savicz, B. ardonense Breidl.) - LTV [244], LAT [3], EST [237], BEL [355], UKR: C [66], M [66], K [328], RUS-EUR: ARC [47, 150], NW [207, 364, 407], KLN [309], C [207, 212, 345], N-UR [90, 150, 207, 415], S-UR [150, 207, 212], CAUC [55, 212, 231, 248], GEO [117], ARM [297], AZE [277], KAZ [157], TUR [296], TAD [296], KYR [351], RUS-AS: W-SIB [265], SSIB [69, 70, 212], E-SIB [69, 212], ARC-YAK [47, 239], YAK [239], ARC-FE [40, 212], S-FE [76]

- alpinum Huds. ex With. (Imbribryum alpinum (Huds. ex With.) N.Pedersen) - UKR: C [66], M [66], K [66, 328], RUS-EUR: SE [207], CAUC [231], GEO [117], ARM [297], KAZ [23], TUR [296], TAD [19, 296], KYR [296], RUS-AS: S-SIB [197, 212], N-FE [140]

- altaicum Broth. - RUS-AS: S-SIB [70] - $\{23\}$

— amblyodon Müll.Hal. - LAT [3], EST [237], BEL [355], UKR: C [66], M [66], K [66, 328], RUSEUR: ARC [47, 150, 415], NW [207, 212, 364, 407], NE [207, 212, 415], KLN [309], C [207, 212, 345], N-UR [90, 207], S-UR [150, 207, 212], CAUC [55, 212, 231, 248], GEO [117], ARM [297], AZE [277], RUS-AS: ARC-WSIB [136], ARC-ESIB [275], S-SIB [69, 197, 212], E-SIB [212, 247, 275], ARC-YAK [47, 239], YAK [239], ARC-FE [40, 212], N-FE [140], S-FE [76, 77, 212] - \{24\}

- amblyphyllum H.Philib. - KYR [296] - \{23\}

- archangelicum Bruch et al. - LAT [3], RUS-EUR: ARC [47, 150, 415], NW [85, 212, 364], N-UR [150, 415], CAUC [231], RUS-AS: ARC-WSIB [136], ARC-EIB [275], S-SIB [275], E-SIB [212, 247, 275], ARC-YAK [47, ?239], YAK [420], N-FE [140] $-\{24\}$ 
- $\operatorname{arcticum}(\mathrm{R}$. Br.) Bruch et al. - LAT [3], EST [237], RUS-EUR: ARC [47, 150, 415], NW [364, 407], NE [207], N-UR [207 ], CAUC [231], RUS-AS: ARC-WSIB [136], ARC-ESIB [164, 212], S-SIB [70, 212], E-SIB [126, 212], ARC-YAK [47, 239], YAK [239], ARC-FE [40, 212], N-FE [40], S-FE $[340, ? 76]-\{25\}$

- arcticum var. kungeanum H.Philib. - KAZ [359]

— argenteum Hedw. - LTV [244], LAT [3], EST [237], BEL [355], UKR: C [66], M [66], K [66, 328], MLD [367], RUS-EUR: ARC [47, 127, 150, 415], NW [207, 212, 364, 407], NE [207, 212, 415], KLN [309], C [207, 212], N-UR [90, 150, 207, 212], S-UR [150, 207, 212], SE [207, 212], CAUC [55, 208, 212, 231, 248], GEO [117], ARM [297], AZE [277], KAZ [23, 159, 296, 356], TUR [296], TAD [294, 296], KYR [267, 296, 351], UZB [267], RUS-AS: ARC-WSIB [136], ARC-ESIB [164, 212, 275], W-SIB [212, 265, 275], S-SIB [69, 70, 212], E-SIB [69, 212, 275], ARC-YAK [47, 212, 239], YAK [239], ARC-FE [40, 212], N-FE [94, 140], S-FE [76, 109, 212, 221]

- argyroglyphodon H.Philib. - KYR [296] - $\{23\}$

- axel-blyttii Kaurin ex H.Philib. (B. acutum Lindb.) - RUS-EUR: ARC [47, 150], NW [364], N-UR [150], S-UR [150], RUS-AS: ARC-WSIB [136], ARC-ESIB [275], E-SIB [275, 420], ARC-YAK [47, $212, ? 239]$, YAK [420] $-\{26\}$

- axillare H.Philib. - KAZ [359] - $\{23\}$

- badium (Bruch ex Brid.) Schimp. - EST [397], BEL [355], UKR: C [66], M [66], K [66, 328], RUSEUR: KLN [309], S-UR [150], GEO [117], ARM [297], AZE [277], RUS-AS: S-SIB [70]

- bimum (Schreb.) Turner (Plagiobryum bimum (Schreb.) N.Pedersen) - LTV [244], LAT [3], BEL [355], UKR: C [66], M [66], K [66, 328], MLD [367], RUS-EUR: ARC [47, 150], NW [207, 364, 407], NE [207], KLN [309], C [207, 212], N-UR [150], S-UR [150, 207, 212], SE [207], CAUC [55, 208, 212, 231], GEO [117], KAZ [23], TAD [296], KYR [296, 351], RUS-AS: ARC-ESIB [275], WSIB [212, 265], S-SIB [69, 70, 212], E-SIB [275, 388], YAK [239], N-FE [140], S-FE [76, 77]

— blindii Bruch et al. - LAT [3], EST [237], GEO [117], KAZ [156, 157], RUS-AS: S-SIB [70] + MIDAS [296]

- bryoides (R. Br.) Wijk \& Margad. - RUS-EUR: NW [364] - $\{25\}$

- bryoides var. terskeanum (H.Philib.) L.I.Savicz - KAZ [157], KYR [296] - \{25\}

- caespiticium Hedw. (incl. B. comense Schimp.) - LTV [244], LAT [3], EST [237], BEL [355], UKR: C [66], M [66], K [66, 328], MLD [367], RUS-EUR: ARC [47, 127, 150, 415], NW [207, 212, 364, 407], NE [207, 212, 415], KLN [309], C [207, 212], N-UR [150, 207, 212, 415], S-UR [150, 207, 212], SE [207, 212], CAUC [55, 208, 212, 231, 248], GEO [117], ARM [297], AZE [277], KAZ [23, 296, 356], TUR [296], TAD [294, 296], KYR [267, 296, 351], RUS-AS: ARC-WSIB [136, 275], ARC-ESIB [212], W-SIB [212, 265], S-SIB [69, 70, 212], E-SIB [212, 247, 275], ARC-YAK [239], YAK [239], ARC-FE [40], N-FE [94, 140], S-FE [212, 221] - $\{27\}$

- calcicola Arnell - RUS-EUR: ARC [47], S-UR [150], RUS-AS: E-SIB [65], ARC-YAK [47, ?239]

- calophyllum R.Br. - LAT [3], EST [237], RUS-EUR: ARC [47], TAD [296], KYR [296], RUSAS: ARC-WSIB [136, 275], ARC-ESIB [420], ARC-YAK [47, ?239], ARC-FE [40, 212], N-FE $[40]-\{26\}$

- capillare Hedw. (Plagiobryum capillare (Hedw.) N.Pedersen, Bryum kaernbachii Müll.Hal.) - LTV [244], LAT [3], EST [237], BEL [355], UKR: C [66], M [66], K [66, 328], MLD [367], RUS-EUR: ARC [47, 150], NW [207, 212, 407], NE [207, 212, 415], KLN [309], C [207, 212], N-UR [90, 150, 207, 212], S-UR [150, 207, 212], SE [207], CAUC [55, 208, 212, 231, 248], GEO [117], ARM [297], AZE [277], KAZ [157, 296, 356], TUR [296], TAD [294, 296], KYR [296, 351], RUS-AS: W-SIB [265], S-SIB [69, 70, 212], E-SIB [69, 212], YAK [239], ARC-FE [40], N-FE [40, 140], S-FE [76, 77, $109,212]$

- caucasicum (Schimp. ex Broth.) C.J.Cox \& Hedd. (Mielichhoferia himalayana Mitt.) - RUS-EUR: CAUC [102, 212, 248], TAD [296]

- congestiflorum H.Philib. - KYR [296] - \{23\}

- crassimucronatum H.Philib. - KYR [296] - $\{23\}$

- creberrimum Taylor - BEL [355], UKR: C [66], M [66], K [66, 328], RUS-EUR: ARC [47, 150, 415], NW [207, 212, 364, 407], NE [207, 212], KLN [309], C [207, 212], N-UR [150, 207, 212, 415], 
S-UR [150, 207, 212, 275], SE [207, 212], CAUC [212, 231, 248], GEO [117], ARM [297], KAZ [23, 296], TAD [296], RUS-AS: ARC-WSIB [136], ARC-ESIB [164, 275], W-SIB [212, 265, 275], SSIB [69, 70, 212], E-SIB [212, 247, 275], YAK [239], ARC-FE [40], N-FE [140], S-FE [37, 212]

- cremocarpum Laz. - TAD [296] - $\{23\}$

- cryophilum Mårtensson (B. tortifolium Brid.) - RUS-EUR: NW [84], N-UR [150, 207], CAUC [212, 231, 248], ARM [297], RUS-AS: ARC-ESIB [164, 212, 275], S-SIB [69, 70, 212], E-SIB [69, 212], ARC-YAK [239], YAK [239], ARC-FE [40], N-FE [94, 140], S-FE [212, 221]

- culmannii Limpr. - RUS-EUR: NW [364]

- cyclophyllum (Schwägr.) Bruch et al. (Plagiobryum cyclophyllum (Schwägr.) N.Pedersen) - LTV [244], LAT [3], BEL [355], RUS-EUR: ARC [47, 150], NW [207, 364, 407], KLN [309], C [207, 212], NUR [150, 207], S-UR [89], RUS-AS: ARC-WSIB [136], ARC-ESIB [212, 275], W-SIB [147, 212], SSIB [197, 212], E-SIB [74, 212], ARC-YAK [47, 239], YAK [239], ARC-FE [38, 212], N-FE [140], SFE [212, 221]

- dichotomum Hedw. (Bryum bicolor Dicks.) - LTV [244], EST [397], BEL [355], UKR: C [66], M [66], RUS-EUR: NE [90, 212], KLN [309], C [207, 345], N-UR [90, 207], S-UR [150, 207], SE [207, 212], CAUC [231, 232, 250], GEO [117], ARM [297], AZE [277], TUR [296], TAD [296], KYR [267, 296], RUS-AS: ARC-ESIB [164], YAK [239], S-FE [76]

- donianum Grev. - AZE [277]

- ehlei Arnell - RUS-AS: ARC-YAK [47, 239] - $\{23\}$

- ekstamii L.I.Savicz - RUS-EUR: ARC [?47, 360, 361]

- elegans Nees (incl. Bryum stirtonii Schimp.) - LAT [3], EST [397], BEL [355], UKR: C [66], M [66], K [66, 328], RUS-EUR: NW [212, 273, 364, 407], NE [207, 212, 415], C [207, 212], N-UR [90, 150, 207, 212], S-UR [150, 207, 212], SE [207], CAUC [55, 212, 231], GEO [117], TUR [296], TAD [296], KYR [267, 296], RUS-AS: ARC-WSIB [?136], ARC-ESIB [164, 420], S-SIB [197, 212], ARCFE [40], N-FE [40, 140], S-FE [76, 109, 212, 221] - \{28\}

- enisseense L.I.Savicz - RUS-AS: E-SIB [360] - \{23, 29$\}$

- funckii Schwägr. - LTV [244], LAT [3], EST [237], BEL [?355], UKR: C [66], M [66], RUS-EUR: NE [207, 212, 416], C [207, 212, 345], S-UR [150, 207, 275], SE [207], CAUC [248], GEO [117], ARM [297], AZE [277], KAZ [23, 296, 356], TUR [296], TAD [293, 296], KYR [296], UZB [267], RUS-AS: ARC-WSIB [136], W-SIB [265], S-SIB [69], E-SIB [74, 212, 275], S-FE [76]

— gemmilucens R.Wilczek \& Demaret - LTV [244], UKR: K [328], TUR [296]

— gemmiparum De Not. - UKR: C [66], K [66, 328], GEO [117], AZE [277], TUR [368]

— intermedium (Brid.) Blandow - LAT [3], EST [237], BEL [355], UKR: C [66], M [66], RUS-EUR: ARC [47, 150, 415], NW [207, 212, 364, 291], NE [207, 212, 415], KLN [309], C [207, 212, 345], NUR [207], S-UR [150, 207, 212], CAUC [231], GEO [117], KAZ [157, 296], KYR [296], RUS-AS: ARC-ESIB [164, 275], W-SIB [265], S-SIB [69], E-SIB [275], ARC-YAK [47, 239], YAK [239], ARC-FE [40], N-FE [140] - $\{30\}$

- jailae Sapjegin - UKR: K [66]

— klinggraeffii Schimp. - LTV [244], EST [237], BEL [355], UKR: C [66], M [398], RUS-EUR: C [207, 420]

- knowltonii Barnes - LTV [244], LAT [3], EST [237], BEL [355], UKR: C [66], RUS-EUR: NW [207, 212, 364, 407], C [207], ?N-UR [150], RUS-AS: ARC-ESIB [420], YAK [239], ARC-FE [420], S-FE [275]

- kunzei Hornsch. - UKR: C [66], M [66], RUS-EUR: C [207, 345], S-UR [150, 207], SE [207, 366], CAUC [208, 212, 231, 248], TAD [294], RUS-AS: S-SIB [70, 212]

- labradorense H.Philib. - RUS-EUR: ARC [47, 150], NW [364], S-UR [150, 212], RUS-AS: ARCWSIB [136]

- laevifilum see B. moravicum

- Iapponicum Kaurin, nom. illeg. - RUS-EUR: NW [364] - $\{31\}$

— leptoglyphodon H.Philib. - KAZ [296, 356], UZB [359], TAD [296, 359], KYR [296, 359]

— leucoglyphodon H.Philib. - KAZ [296], KYR [296] - \{23\}

— lonchocaulon Müll.Hal. (B. saxatile I.Hagen) - BEL [355*], UKR: C [66], M [66], K [66, 328], MLD 
[367], RUS-EUR: NW [207, 364, 407], NE [207], C [207, 212], N-UR [207], S-UR [207, 212], SE [207, 212], CAUC [231], GEO [117*], AZE [277], KAZ [296, 356], TAD [294, 296], KYR [296], RUS-AS: ARC-ESIB [275], W-SIB [147, 212], S-SIB [69, 70, 212], E-SIB [69, 212, 275], YAK [239], N-FE [140] - $\{32\}$

- longisetum Blandow ex Schwägr. - BEL [355], RUS-EUR: NW [364], KLN [309], C [423], RUSAS: S-SIB [420]

- marratii Hook. f. \& Wilson - LAT [3], EST [237], RUS-AS: S-SIB [420]

- mayrii Broth. - RUS-AS: S-FE [67, 361]

- microcalophyllum H.Philib. - KYR [296] - $\{23\}$

— mildeanum Jur. - UKR: C [66], RUS-EUR: NW [364], CAUC [232], GEO [117], MID-AS [296], RUS-AS: S-SIB [420]

- mirabile Müll.Hal. - RUS-AS: ARC-FE [40]

- mollifolium H.Philib. - KYR [296] - $\{23\}$

- moravicum Podp. (B. laevifilum Syed, B. subelegans auct. non Kindb.) - LTV [244], LAT [3], EST [237, 397], BEL [355], UKR: [66], K [328], MLD [367], RUS-EUR: NW [207, 212, 289, 364], NE [207, 212], KLN [309], C [207, 212], N-UR [90, 150, 207, 212], S-UR [150, 207, 212], SE [207, 212], CAUC [55, 212, 231, 248], GEO [117], ARM [297], AZE [277], KAZ [296, 356], TUR [368], TAD [296], KYR [267, 296], UZB [267], RUS-AS: S-SIB [70, 212], E-SIB [388], YAK [239], N-FE [40, 140], S-FE [212, 77, 221]

- mucronifolium H.Philib. - KYR [296] - \{23\}

- muehlenbeckii Bruch et al. (Imbribryum muehlenbeckii (Bruch et al.) N.Pedersen) - RUS-EUR: NW [364, 407], CAUC [231], GEO [117], RUS-AS: S-SIB [212, 334]

- murale Wilson ex Hunt - GEO [117]

- murmanicum Broth. - RUS-EUR: NW [85, 212, 364]

- neodamense Itzigs. - LTV [244], LAT [3], EST [237], BEL [355], RUS-EUR: ARC [47, 150, 415], NW [364], KLN [309], N-UR [150, 415], CAUC [231], RUS-AS: ARC-WSIB [?136], ARC-ESIB [164, 275, 420], W-SIB [265], S-SIB [70], E-SIB [275, 388], ARC-YAK [47, 239], YAK [239], N-FE [420], S-FE [76] - $\{33\}$

— nitidulum Lindb. - RUS-EUR: ARC [47], RUS-AS: ARC-WSIB [?47], ARC-ESIB [?47], ARC-YAK [47, ?239], ARC-FE [40, 212] - $\{30\}$

- oblongum Lindb. - LAT [3], RUS-EUR: NW [85, 212, 364], NE [207], C [420], RUS-AS: E-SIB [275], YAK [239]

- obtusidens Arnell - RUS-AS: ARC-YAK [47, 239] - $\{23\}$

- pallens Sw. ex anon. (Plagiobryum pallens (Sw. ex anon.) N.Pedersen) - LTV [244], LAT [3], EST [237], BEL [355], UKR: C [66], M [66], K [66, 328], RUS-EUR: ARC [47, 150, 415], NW [207, 212, 364, 407], NE [207, 212, 415], KLN [309], C [207, 212, 345], N-UR [150, 207, 415], S-UR [150, 207, 212], SE [207], CAUC [212, 231, 248], GEO [117], ARM [297], AZE [277], KAZ [296, 356], TAD [296], RUS-AS: ARC-WSIB [136], ARC-ESIB [164], W-SIB [212, 265], S-SIB [70, 212], E-SIB [275, 388], ARC-YAK [47, 239], YAK [239], ARC-FE [40], N-FE [140], S-FE [76, 109, 212]

- pallescens Schleich. ex Schwägr. (Plagiobryum pallescens (Schleich. ex Schwägr.) N.Pedersen, $B$. obconicum Hornsch. ex Bruch et al.) - LTV [244], LAT [3], EST [237], BEL [355*], UKR: C [66], M [66], K [66, 328], RUS-EUR: ARC [47, 127, 150], NW [212, 364, 407], NE [207, 415], KLN [309], C [207, 212], N-UR [150, 207], S-UR [150, 207, 212], SE [207], CAUC [212, 231], GEO [117*], ARM [297], AZE [277], KAZ [157, 296], TUR [296], TAD [296], KYR [296], UZB [267], RUSAS: ARC-WSIB [136], W-SIB [265], S-SIB [69, 70, 212], E-SIB [212, ?247], ARC-YAK [47, ?239], YAK [?239], ARC-FE [40], N-FE [140] - \{32\}

— pallescens var. microblastum (Müll.Hal.) Podp. - RUS-AS: ARC-FE [40] - $\{23\}$

- pallescens var. pootonense Podp. - RUS-AS: ARC-FE [40] - $\{23\}$

- pallescens var. turkestanicum (Podp.) L.I.Savicz - KYR [296] - $\{23\}$

— pamirense H.Philib. ex Broth. - RUS-EUR: ?NE [416], TAD [296] — \{23\}

- pamiro-mucronatum H.Philib. ex Broth. - TAD [296] - \{23\}

- pseudotriquetrum (Hedw.) P.Gaertn., B.Mey. \& Scherb. (Plagiobryum pseudotriquetrum (Hedw.) 
N.Pedersen) - LTV [244], LAT [3], EST [237], BEL [355], UKR: C [66], M [66], K [66, 328], RUSEUR: ARC [47, 127, 150], NW [207, 212, 364, 407], NE [207, 212, 415], KLN [309], C [207, 212], N-UR [150, 207, 212], S-UR [150, 207, 212], SE [207], CAUC [55, 212, 231, 248], GEO [117], ARM [297], AZE [277], KAZ [23, 296, 356], TAD [294, 296], KYR [296], UZB [267], RUS-AS: ARCWSIB [136], ARC-ESIB [164, 212], W-SIB [212, 265], S-SIB [69, 70, 212], E-SIB [69, 212], ARCYAK [47, 212, 239], YAK [212, 239], ARC-FE [40, 212], N-FE [140], S-FE [76, 77, 212]

- purpurascens (R. Br.) Bruch et al. (Plagiobryum purpurascens (R. Br.) N.Pedersen, B. serotinum Lindb.) - RUS-EUR: ARC [47, 150, 415], NW [85, 212, 364], NE [207, 415], KYR [296], RUS-AS: ARCWSIB [136], ARC-ESIB [212, 263, 275], W-SIB [212, 265], S-SIB [69], ARC-YAK [?47], YAK [?239], ARC-FE [?40] - $\{25\}$

- pygmaeomucronatum H.Philib. - KYR [296] - \{23\}

— radiculosum Brid. - RUS-EUR: CAUC [231, 232, 250], ARM [297], RUS-AS: S-FE [420]

— rubens Mitt. - LTV [244], LAT [3], EST [396], BEL [355], UKR: C [66, 398], M [66, 398], RUS-EUR: NW [420], KLN [309], C [207, 345], N-UR [207], SE [207], CAUC [232, 420], ARM [297], AZE [361]

— ruderale Crundw. \& Nyholm - LTV [244], UKR: M [398], AZE [277]

- rutilans Brid. - RUS-EUR: ARC [47, 150], NW [85, 212, 291, 364], NE [415], N-UR [150], TUR [296], RUS-AS: ARC-ESIB [47, 50, 275], ARC-YAK [49, 239], ARC-FE [40, 140]

- salinum I.Hagen ex Limpr. - EST [237], RUS-EUR: ARC [47], NW [85, 212, 291, 364], S-UR [373], RUS-AS: ARC-ESIB [420], YAK [239], S-FE [109]

- sauteri Bruch et al. - RUS-EUR: C [419], CAUC [102]

— savicziae Schljakov - RUS-EUR: NW [362], RUS-AS: S-SIB [420]

- schleicheri DC. - LTV [244], BEL [355], UKR: C [66], RUS-EUR: ARC [47, 150], NW [364], NE [416], C [207], N-UR [150], CAUC [55, 212, 231, 248], GEO [117], ARM [297], AZE [277], KAZ [156, 159, 296, 356], TUR [296], TAD [294, 296], KYR [267, 296, 351], UZB [267], RUS-AS: ARCESIB [164], W-SIB [212, 265], S-SIB [70, 212], E-SIB [163, 388], ARC-FE [40], N-FE [140], S-FE $[67$, as B. turbinatum] $-\{34\}$

— sibiricum Lindb. \& Arnell - RUS-AS: E-SIB [275], YAK [?239]

- spinifolium H.Philib. - KYR [296] - $\{23\}$

- subapiculatum Hampe (B. erythrocarpum auct. partim, B. microerythrocarpum Müll.Hal. \& Kindb. ex Macoun) - LTV [244], EST [237], UKR: M [398], K [328, 398], RUS-EUR: NW [207, 420], C [207, 212, 345], S-UR [150], SE [207, 212, 366], GEO [117], RUS-AS: S-FE [79, 212]

- subcalophyllum (H.Philib.) Paris - KYR [296] - $\{23\}$

- submucronatum H.Philib. - KYR [296] - \{23\}

— taimyrense Broth. \& Bryhn - RUS-AS: ARC-ESIB [361], ARC-YAK [47, ?239]

- tardum Bomanss. - excluded - $\{34\}$

- tenuisetum Limpr. - UKR: M [405] - $\{34\}$

- teres Lindb. - RUS-EUR: ARC [47], NW [364], RUS-AS: ARC-WSIB [136], ARC-ESIB [164, 212], ARC-YAK [47, 239], YAK [239], ARC-FE [40, 212]

- terskeiense Paris (B. paradoxum H.Philib.) - KYR [296] - $\{23\}$

- tesselatum H.Philib. - KYR [296] - $\{23\}$

— timmiostomoides H.Philib. - KAZ [296, 356], KYR [296] - $\{23\}$

- torquescens Bruch et al. - UKR: K [66, 328], RUS-EUR: CAUC [212, 232], GEO [117], ARM [297], AZE [277], TUR [368], TAD [296], KYR [296]

- turbinatum (Hedw.) Turner (B. planiusculum Lindb. \& Arnell) - LTV [244], LAT [3], EST [396], BEL [355], UKR: C [66], M [66], K [66, 328], RUS-EUR: ARC [47, 150], NW [207], NE [207], KLN [309], C [207, 212], N-UR [150], S-UR [150, 207, 212], SE [207], CAUC [55, 212, 232, 248], GEO [117], ARM [297], AZE [277], UZB [347], TAD [296], KYR [267, 296], RUS-AS: E-SIB [388], YAK [239] - $\{34\}$

- uliginosum (Brid.) Bruch et al. (Plagiobryum uliginosum (Brid.) N.Pedersen) - EST [237], BEL [355], UKR: C [66], M [66], RUS-EUR: NW [207, 283, 364], NE [207, 212], KLN [309], C [207, 212], CAUC [212, 248], GEO [117], ARM [297], TAD [296], RUS-AS: W-SIB [265], S-SIB [197, 212], ESIB [388], N-FE [140], S-FE [77, 109, 212] 
— umbratum I.Hagen - RUS-EUR: NW [364]

- utriculatum Müll.Hal. - RUS-AS: ARC-FE [40] - $\{23\}$

- vernum H.Philib. - KYR [296] - $\{23\}$

- veronenese De Not. - UKR [66]

— violaceum Crundw. \& Nyholm - LTV [244], UKR: M [398], RUS-EUR: NW [283], C [207, 212, 345], RUS-AS: W-SIB [420]

— warneum (Röhl.) Brid. (B. mamillatum Lindb., B. oelandicum H.Philib.) - LAT [3], EST [237], BEL [355], RUS-EUR: KLN [309], C [207], RUS-AS: S-SIB [69, 70], YAK [239]

- weigelii Spreng. - LTV [244], LAT [3], EST [237], BEL [355], UKR: C [66], M [66], RUS-EUR: ARC [47, 150], NW [207, 212, 364, 407], NE [207, 212, 415], C [207, 212, 345], N-UR [150, 207, 212], S-UR [150, 207, 212], CAUC [231], GEO [117], ARM [297], AZE [277], KYR [296], RUSAS: ARC-ESIB [93], W-SIB [265], S-SIB [197, 212], E-SIB [275], ARC-YAK [47], YAK [239], ARC-FE [40], N-FE [94, 140], S-FE [114]

- wrightii Sull. (Plagiobryum wrightii (Sull.) N.Pedersen) - RUS-EUR: ARC [47, 150], RUS-AS: ARCWSIB [136], ARC-ESIB [164, 212, 275], E-SIB [163, 275], ARC-YAK [47, 239], YAK [?239], ARCFE [40, 212], N-FE [40]

— zemliae Arnell \& Jäderh. - RUS-EUR: ARC [47] - $\{23\}$

Bucklandiella Roiv. [Grimmiaceae]

- affinis (F.Weber \& D.Mohr) Bednarek-Ochyra \& Ochyra (Racomitrium affine (Schleich. ex F. Weber \& D.Mohr) Lindb.) - UKR: [66], RUS-EUR: NW [407], RUS-AS: S-SIB [70]

- afoninae (Frisvoll) Bednarek-Ochyra \& Ochyra (Racomitrium afoninae Frisvoll) - RUS-AS: ARC-FE $[40,212]$

- heterosticha (Hedw.) Bednarek-Ochyra \& Ochyra (Racomitrium heterostichum (Hedw.) Brid.) - LTV [244], LAT [3], EST [237], BEL [355], UKR: C [66], M [66], K [66, 328], RUS-EUR: NW [207, 407], NE [212], ?N-UR [415], KLN [309], C [207, 212], GEO [117] - \{35\}

- Iaeta (Besch. \& Cardot) Bednarek-Ochyra \& Ochyra (Racomitrium laetum Besch. \& Cardot) - RUSAS: S-FE [212, 221]

- macounii (Kindb.) Bednarek-Ochyra \& Ochyra subsp. alpina (E.Lawton) Bednarek-Ochyra \& Ochyra (Racomitrium macounii subsp. alpinum (E.Lawton) Frisvoll) - RUS-EUR: CAUC [55, 212, 231]

- microcarpa (Hedw.) Bednarek-Ochyra \& Ochyra (Racomitrium microcarpon (Hedw.) Brid.) - LAT [3], EST [237], BEL [355], UKR: C [66], RUS-EUR: ARC [47, 127, 150, 415], NW [207, 212, 364, 407], C [207, 212], N-UR [90, 150, 207, 212, 415], S-UR [150, 207, 212], RUS-AS: S-SIB [69, 70, 202, 212], E-SIB [74, 212, 247], YAK [239], ARC-FE [40], N-FE [140], S-FE [76, 212, 221]

- microcarpa fo. afoninae (Frisvoll) Bednarek-Ochyra \& Ochyra - RUS-AS: ARC-FE [40]

- nitidula (Cardot) Bednarek-Ochyra \& Ochyra - RUS-AS: N-FE [140]

- sudetica (Funck) Bednarek-Ochyra \& Ochyra (Racomitrium sudeticum (Funck) Bruch et al.) - EST [397], UKR: C [66], K [66, 328], RUS-EUR: ARC [47, 150], NW [212, 273, 364, 407], N-UR [150, 207, 212, 415], S-UR [150, 207], CAUC [55, 212, 231, 248], GEO [18, 117], ARM [297], RUS-AS: ARC-ESIB [212, 263], S-SIB [69, 70, 202, 212], E-SIB [74, 212, 247], ARC-YAK [47], YAK [239], ARC-FE [40], N-FE [140], S-FE [212, 221]

- sudetica fo. terricola (Frisvoll) Bednarek-Ochyra \& Ochyra - RUS-AS: ARC-FE [40]

Buxbaumia Hedw. [Buxbaumiaceae]

- aphylla Hedw. - LTV [244], LAT [3], EST [237], BEL [355], UKR: C [66], M [66], RUS-EUR: ARC [47, 127, 150], NW [207, 212, 364, 407], NE [207, 212, 415], KLN [309], C [207, 212], N-UR [150, 207, 212], S-UR [150, 207, 212], SE [207], GEO [117], RUS-AS: ARC-WSIB [136], W-SIB [212, 265], SSIB [69, 70, 212], E-SIB [69, 275], YAK [239], ARC-FE [40], N-FE [94, 110, 140], S-FE [109, 212, 221]

- minakatae S.Okamura - RUS-AS: S-SIB [69, 70, 197, 212], S-FE [76, 212]

— viridis (Moug. ex Lam. \& DC.) Brid. ex Moug. \& Nestl. - LTV [244], LAT [3], EST [237], UKR: C [66], M [66], K [328], RUS-EUR: KLN [309], NW [261], CAUC [55, 212, 231], GEO [117], AZE [277]

Callialaria see Cratoneuron

Callicladium H.A.Crum [Pylaisiaceae]

— haldanianum (Grev.) H.A.Crum (Heterophyllium haldanianum (Grev.) Kindb.) - LTV [244], LAT 
[3], EST [237], BEL [355], UKR: C [66], M [66], RUS-EUR: ARC [47, 150], NW [207, 212, 407], NE [207, 212, 415], KLN [309], C [207, 212], N-UR [90, 150, 207, 415], S-UR [150, 207, 212], SE [207], CAUC [55, 212, 231, 248], GEO [117], KAZ [296, 356], RUS-AS: W-SIB [212, 265, 275], S-SIB [69, 70, 212], E-SIB [69, 212, 275], ARC-FE [40], N-FE [140], S-FE [76, 77, $109,212]$

Calliergon (Sull.) Kindb. [Calliergonaceae], see also Straminergon stramineum, Warnstorfia sarmentosa, Pseudocalliergon trifarium

- cordifolium (Hedw.) Kindb. - LTV [244], LAT [3], EST [237], BEL [355], UKR: C [66], M [66], RUS-EUR: ARC [47, 127, 150], NW [207, 212, 364, 407], NE [207, 212, 415], KLN [309], C [207, 212], N-UR [150, 207, 212], S-UR [150, 207, 212], SE [207, 212], CAUC [55, 212, 231, 248], GEO [117], ARM [297], AZE [277], KAZ [296], TAD [26, 296], RUS-AS: ARC-WSIB [136], ARC-ESIB [164, 212, 275], W-SIB [212, 265], S-SIB [69, 70, 212], E-SIB [69, 212, 275], ARC-YAK [47, 212 , 239], YAK [212, 239], ARC-FE [40, 212], N-FE [94, 140], S-FE [76, 77, 109, 212]

- giganteum (Schimp.) Kindb. - LTV [244], LAT [3], EST [237], BEL [355], UKR: C [66], M [66], RUS-EUR: ARC [47, 127, 150], NW [207, 212, 364, 407], NE [207, 212, 415], KLN [309], C [207, 212], N-UR [150, 207, 212], S-UR [150, 207, 212], SE [207], CAUC [55, 212], GEO [117], AZE [277], KAZ [296, 356], KYR [296], RUS-AS: ARC-WSIB [136], ARC-ESIB [164, 212, 275], W-SIB $[212,265]$, S-SIB [69, 70, 197, 212], E-SIB [69, 212, 275], ARC-YAK [47, 212, 239], YAK [212, 239], ARC-FE [40, 212], N-FE [94, 140], S-FE [76, 109, 212, 221]

- megalophyllum Mikut. - LAT [3], EST [237], RUS-EUR: ARC [47, 127], NW [207, 407], NE [207], C [207], RUS-AS: ARC-WSIB [136], ARC-ESIB [164, 212], W-SIB [212, 265], E-SIB [163], ARCYAK [47, 212, 239], YAK [239], ARC-FE [40], N-FE [94, 140]

- richardsonii (Mitt.) Kindb. - LTV [244], LAT [3], EST [237], UKR: C [66], RUS-EUR: ARC [47, 127, 150], NW [207, 212, 364, 407], NE [207, 212, 415], C [207, 212], N-UR [150, 207, 212], CAUC [212, 248], GEO [117], KAZ [296], RUS-AS: ARC-WSIB [136], ARC-ESIB [164, 212, 275], W-SIB $[212,265]$, S-SIB [69, 70, 212], E-SIB [212, 275], ARC-YAK [47, 212, 239], YAK [239], ARC-FE [40, 212], N-FE [94, 140], S-FE [114, 212, 221]

Calliergonella Loeske [Pylaisiaceae] - $\{36\}$

- cuspidata (Hedw.) Loeske - LTV [244], LAT [3], EST [237], BEL [355], UKR: C [66], M [66], K [66, 328], MLD [367], RUS-EUR: ARC [47, 150], NW [207, 212, 364, 407], NE [207, 212, 415], KLN [309], C [207, 212], N-UR [150, 207, 212], S-UR [150, 207, 212], CAUC [212, 231, 248], GEO [117], ARM [297], AZE [277], KAZ [296], UZB [267], KYR [296], RUS-AS: ARC-WSIB [136], ARC-ESIB [93, 96, 212], W-SIB [265], S-SIB [69, 70, 212], E-SIB [212, 247], YAK [212, 239], ARCFE [40], N-FE [94, 140], S-FE [76, 77, 109, 212]

- lindbergii (Mitt.) Hedenäs (Hypnum lindbergii Mitt., Breidleria arcuata (Molendo) Loeske) - LTV [244], LAT [3], EST [237], BEL [355], UKR: C [66], M [66], RUS-EUR: ARC [47, 127, 150], NW [207, 212, 364, 407], NE [207, 212, 415], KLN [309], C [207, 212], N-UR [150, 207, 212], S-UR [150, 207, 212], SE [207], CAUC [55, 212, 231, 248], GEO [117], ARM [297], AZE [277], KAZ [296, 356], RUS-AS: ARC-WSIB [136], ARC-ESIB [164, 212, 275], W-SIB [212, 265], S-SIB [69, 70, 212], E-SIB [69, 212, 275], ARC-YAK [47, 212, 239], YAK [212, 239], ARC-FE [40, 212], N-FE $[94,140]$, S-FE $[76,77,109,212]$

Camptothecium see Homalothecium lutescens, Tomentypnum nitens (=Campthothecium trichoides)

Campyliadelphus (Kindb.) R.S.Chopra [Amblystegiaceae]

- chrysophyllus (Brid.) R.S.Chopra (Campylium chrysophyllum (Brid.) Lange) - LTV [244], LAT [3], EST [237], BEL [355], UKR: C [66], M [66], K [66, 328], MLD [367], RUS-EUR: ARC [47, 150], NW [207, 273, 364, 407], NE [207, 212, 415], KLN [309], C [207, 212], N-UR [150, 207, 212], S-UR [150, 207, 212], SE [207, 212], CAUC [55, 212, 231, 248], GEO [117], ARM [297], AZE [277], KYR [296], RUS-AS: ARC-WSIB [136], ARC-ESIB [47, 275], W-SIB [265], S-SIB [69, 70, 212], ESIB [212, 275], ARC-YAK [47, 212, 239], YAK [212, 239], ARC-FE [40, 212], N-FE [140], S-FE [76, $77,114,212]$

- elodes (Lindb.) Kanda (Campylium elodes (Lindb.) Kindb.) - LTV [244], LAT [3], EST [237], BEL [355], UKR: C [66], M [66], ARM [297], RUS-EUR: NW [407], RUS-AS: S-FE [76] 
Campylidium (Kindb.) Ochyra [Amblystegiaceae] - $\{37\}$

- calcareum (Crundw. \& Nyholm) Ochyra (Campylium calcareum Crundw. \& Nyholm, Campylophyllum calcareum (Crundw. \& Nyholm) Hedenäs ) - LTV [244], LAT [3], EST [237], UKR: C [66], M [66], K [66, 328], RUS-EUR: NW [63, 291, 364], NE [416], C [207, 212, 345], N-UR [207], S-UR [212, 422], SE [207], RUS-AS: YAK [239], ARC-FE [40], N-FE [40]

- hispidulum (Brid.) Ochyra (Campylium hispidulum (Brid.) Mitt., Campylophyllum hispidulum (Brid.) Hedenäs - RUS-EUR: ?ARC [47, 150], ?NW [212], ?C [212, 345], ?N-UR [150], ?S-UR [150, 212], ?GEO [117], ?KAZ [296], RUS-AS: ?ARC-WSIB [136], ?W-SIB [212, 265], ?S-SIB [69, 70, 212], ?E-SIB [69, 212], ?ARC-YAK [47, 239], ?YAK [212, 239], ?ARC-FE [40], ?N-FE [94, 140], ?S-FE $[76,77,109,212]-\{38\}$

- sommerfeltii (Myrin) Ochyra (Campylium sommerfeltii (Myrin) Lange, Campylophyllum sommerfeltii (Myrin) Hedenäs) - LTV [244], LAT [3], EST [237], BEL [355], UKR: C [66], M [66], K [66, 328], MLD [367], RUS-EUR: ARC [47, 150], NW [207, 212, 364, 407], NE [207, 212, 415], KLN [309], C [207, 212], N-UR [150, 207, 212], S-UR [150, 207, 212], SE [207, 212], CAUC [231, 232, 248], GEO [117], ARM [297], AZE [277], RUS-AS: W-SIB [265], S-SIB [69, 70, 212], E-SIB [69, 212], ARCYAK [239], YAK [212, 239], N-FE [40, 140], S-FE [76, 109, 212]

Campylium (Sull.) Mitt. [Amblystegiaceae], see also Amblystegium radicale, Campylidium sommerfeltii, etc., Campyliadelphus chrysophyllus, etc., Campylophyllum halleri, Drepanocladus polygamus, D. arcticus (=Campylium zemliae), Podperaea krylovii

- longicuspis (Lindb. \& Arnell) Hedenäs - RUS-AS: ARC-WSIB [136], ARC-ESIB [47, 275], S-SIB [197], E-SIB [163, 275], ARC-YAK [47, 239], ARC-FE [40] - \{39\}

- protensum (Brid.) Kindb. (C. stellatum subsp. protensum (Brid.) C.E.O.Jensen, C. stellatum var. protensum (Brid.) Bryhn) - LTV [244], LAT [1], EST [237], BEL [355], UKR: C [66], M [66], K [328], MLD [367], RUS-EUR: ARC [47], NW [212, 273, 364, 407], NE [414], C [207, 212, 345], N-UR [150, 207, 212], S-UR [150, 207, 212], CAUC [55, 212, 231, 248], GEO [117], ARM [297], AZE [277], RUS-AS: ARC-ESIB [164, 212, 275], W-SIB [265], S-SIB [69, 70, 212], E-SIB [212, 275], ARC-YAK [47, 239], YAK [212, 239], ARC-FE [40, 212], N-FE [40, 140], S-FE [79, 212]

- squarrosulum (Besch. \& Cardot) Kanda - RUS-AS: S-FE [78, 178] - $\{40\}$

— stellatum (Hedw.) C.E.O.Jensen - LTV [244], LAT [3], EST [237], BEL [355], UKR: C [66], M [66], K [66, 328], MLD [367], RUS-EUR: ARC [47, 127, 150], NW [207, 212, 364, 407], NE [207, 212, 415], KLN [309], C [207, 212], N-UR [150, 212], S-UR [150, 212], CAUC [55, 212], GEO [117], ARM [297], AZE [277], KAZ [156, 159, 296, 356], TAD [26, 296], KYR [296], RUS-AS: ARC-WSIB [136], ARC-ESIB [164, 212, 275], W-SIB [265], S-SIB [69, 70, 212], E-SIB [69, 212, 275], ARC-YAK [47, 212, 239], YAK [212, 239], ARC-FE [40, 212], N-FE [94, 140], S-FE [76, 109, 212, 221] — 441$\}$

Campylophyllum (Schimp.) M.Fleisch. [Amblystegiaceae]

- halleri (Hedw.) M.Fleisch. (Campylium halleri (Hedw.) Lindb.) - EST [237], UKR: C [66], RUSEUR: NW [364, 407], N-UR [150, 207, 212, 415], S-UR [150, 207, 212], CAUC [55, 212], GEO [13, 117], ARM [297], RUS-AS: ARC-ESIB [93], S-SIB [69, 70, 212], YAK [212, 239], S-FE [114]

Campylopus Brid. [Leucobryaceae]

— atrovirens De Not. - RUS-AS: N-FE [140]

- flexuosus (Hedw.) Brid. - RUS-EUR: KLN [309], GEO [117]

- fragilis (Brid.) Bruch et al. - UKR: C [66], M [66], RUS-EUR: CAUC [102, 248], RUS-AS: S-SIB [197, 212], S-FE [178]

- gracilis (Mitt.) A.Jaeger (C. schwarzii Schimp.) - UKR: C [66]

— introflexus (Hedw.) Brid. - LTV [244], LAT [5], RUS-EUR: KLN [309, 352]

— pyriformis (Schultz) Brid. - EST [237], RUS-EUR: KLN [309], GEO [31, 117], RUS-AS: S-FE [76, 268]

- schimperi Milde (C. subulatus var. schimperi (Milde) Husn.) - RUS-EUR: CAUC [55, 102, 212, 248], GEO [102], RUS-AS: ARC-ESIB [?47], S-SIB [197, 212], ARC-FE [40], N-FE [140], S-FE $[212,221]$

— subulatus Schimp. ex Milde - RUS-EUR: NW [364], ?CAUC [31, 231], RUS-AS: ARC-FE [40], NFE [140]

— umbellatus (Arn.) Paris - RUS-AS: N-FE [235] 
Campylostelium Bruch et al. [Ptychomitriaceae]

- pitardii (Corb.) E.Maier (Grimmia pitardii Corb., Usmania campylopoda Laz.) - TUR [296], UZB [306], TAD $[295,296]-\{42\}$

- saxicola (F.Weber \& D.Mohr) Bruch et al. - UKR: C [66, 413]

- strictum Solms - UKR: C [66, 413] - $\{43\}$

Catoscopium Brid. [Catoscopiaceae]

- nigritum (Hedw.) Brid. - LAT [3], EST [237], BEL [355], RUS-EUR: ARC [47, 414], NW [207, 212, 364, 407], NE [207, 415], C [207], GEO [13], RUS-AS: ARC-WSIB [136], ARC-ESIB [164, 275], S-SIB [69, 70, 212], ARC-YAK [47, 212, 239], YAK [212, 239], ARC-FE [40, 212], N-FE [40, 94], S-FE [114]

Ceratodon Brid. [Ditrichaceae]

- conicus (Hampe) Lindb. (C. purpureus var. conicus (Hampe) Husn.) - EST [237], RUS-EUR: CAUC [102], GEO [102, 117]

- heterophyllus Kindb. (C. purpureus var. rotundifolius Berggr.) - RUS-EUR: ARC [42], RUS-AS: ARC-WSIB [47], ARC-ESIB [47, 50, 164], ARC-YAK [239], ARC-FE [40], N-FE [140] - \{44\}

- purpureus (Hedw.) Brid. - LTV [244], LAT [3], EST [237], BEL [355], UKR: C [66], M [66], K [328], MLD [367], RUS-EUR: ARC [47, 127, 150], NW [207, 212, 364, 407], NE [207, 212, 415], KLN [309], C [207, 212], N-UR [150, 207, 212], S-UR [150, 207, 212], SE [207, 212], CAUC [55, 208, 212, 231, 248], GEO [117], ARM [297], AZE [277], KAZ [296, 356], TUR [296], TAD [294, 295], KYR [351], RUS-AS: ARC-WSIB [136], ARC-ESIB [164, 212], W-SIB [212, 265], S-SIB [69, 70, 212], E-SIB [69, 212, 275], ARC-YAK [47, 212, 239], YAK [212, 239], ARC-FE [40, 212], N-FE $[94,140]$, S-FE $[76,77,109,212]$

Cinclidium Sw. [Mniaceae]

- $\operatorname{arcticum}$ (Bruch et al.) Schimp. - RUS-EUR: ARC [47], N-UR [150, 415], ARM [297], RUS-AS: ARC-WSIB [136], ARC-ESIB [164, 212, 275], S-SIB [69, 70, 212], E-SIB [126, 212, 275], ARCYAK $[47,212,239]$, YAK $[212,239]$, ARC-FE [40, 212], N-FE [40, 94]

- latifolium Lindb. (C. minutifolium Broth.) - KAZ [296, 356], RUS-AS: ARC-WSIB [136], ARCESIB [164, 212], E-SIB [126, 212], ARC-YAK [47, 212, 239], YAK [239], ARC-FE [40, 212], N-FE $[94,140]-\{45\}$

- stygium Sw. - LTV [244], LAT [3], EST [237], BEL [355], UKR: C [66], RUS-EUR: ARC [47, 150], NW [207, 212, 364, 407], NE [207, 212, 415], C [207, 345], N-UR [90, 150, 207, 212], RUSAS: ARC-WSIB [136], ARC-ESIB [96, 212], W-SIB [265], S-SIB [70, 212], E-SIB [126, 212], ARCYAK [47, 239], YAK [212, 239], ARC-FE [40], N-FE [40, 140]

- subrotundum Lindb. - RUS-EUR: ARC [47, 127, 150], NW [212, 273, 364, 407], RUS-AS: ARCWSIB [136], ARC-ESIB [164, 212, 275], E-SIB [126, 212], ARC-YAK [47, 212, 239], YAK [212, 239], ARC-FE [40, 212], N-FE [94, 140]

Cinclidotus P.Beauv. [Pottiaceae]

- aquaticus (Hedw.) Bruch et al. - UKR: K [66, 328]

- danubicus Schiffn. \& Baumgartner - LAT [3], BEL [355]

- fontinaloides (Hedw.) P.Beauv. - UKR: C [66], M [66], K [66, 328], RUS-EUR: CAUC [55, 208, 212], GEO [117], AZE [277], KAZ [157, 393], KYR [296], UZB [267]

- riparius (Host ex Brid.) Arn. - UKR: K [328], RUS-EUR: CAUC [55, 212], GEO [117], ARM [297], KAZ [156, 157, 159], UZB [347], TAD [294, 295], KYR [296, 349, 351], RUS-AS: S-SIB $[70,197]$

Cirriphyllum Grout [Brachytheciaceae], see also Brachythecium cirrosum, B. tommasinii (=C. tenuinerve, C. vaucheri), Sciuro-hypnum flotowianum (=C. velutinoides, C. reichenbachianum)

- crassinervium (Taylor) Loeske \& M.Fleisch. (Eurhynchium crassinervium (Taylor) Schimp.) - UKR: C [66], M [66], K [66, 328], RUS-EUR: CAUC [55, 208, 212, 232, 248], GEO [117], ARM [297], AZE [277], TUR [296], KYR [350]

— piliferum (Hedw.) Grout - LTV [244], LAT [3], EST [237], BEL [355], UKR: C [66], M [66], MLD [367], RUS-EUR: ARC [150], NW [207, 212, 364, 407], NE [207, 212, 415], KLN [309], C [207, 212], N-UR [150, 207, 212, 415], S-UR [150, 207, 212], CAUC [55, 212, 231, 248], GEO [117], 
ARM [297], AZE [277], KAZ [156], KYR [296], RUS-AS: W-SIB [212, 265], S-SIB [69, 70, 198, 212], E-SIB [69, 212, 275], S-FE [77, 212]

Claopodium (Lesq. \& James) Renauld \& Cardot [*Leskeaceae]

- crispifolium (Hook.) Renauld \& Cardot - excluded - $\{46\}$

— pellucinerve (Mitt.) Best - RUS-AS: S-SIB [69, 70, 212], E-SIB [212, 275], YAK [239], N-FE [140], S-FE [76, 77, 109, 212]

- rostratum (Hedw.) Ignatov, comb. nov. - Leskea rostrata Hedw., Spec. Musc. Frond. 225. tab. 55 figs. 13-18. 1801. (Anomodon rostratus (Hedw.) Schimp.) - UKR: C [66], RUS-EUR: CAUC [55, 102, 212], GEO [117] - $\{47\}$

Cleistocarpidium Ochyra \& Bednarek-Ochyra [Ditrichaceae]

— palustre (Bruch \& Schimp.) Ochyra \& Bednarek-Ochyra (Pleuridium palustre (Bruch \& Schimp.) Bruch et al., Sporledera palustris (Bruch \& Schimp.) Hampe) - UKR: C [66], M [66] + CAUCASUS [341]

Climacium F.Weber \& D.Mohr [Climaciaceae]

— dendroides (Hedw.) F.Weber \& D.Mohr - LTV [244], LAT [3], EST [237], BEL [355], UKR: C [66], M [66], K [66, 328], MLD [367], RUS-EUR: ARC [47, 127, 150], NW [207, 212, 364, 407], NE [207, 212, 415], KLN [309], C [207, 212], N-UR [150, 207, 212], S-UR [150, 207, 212], SE [207, 212], CAUC [55, 212, 231, 248], GEO [117], ARM [297], AZE [277], KAZ [156, 159, 296, 356], TAD [26, 296], KYR [296, 351], RUS-AS: ARC-WSIB [136], W-SIB [212, 265], S-SIB [69, 70, 212], E-SIB [69, 212, 275], ARC-YAK [47, 212, 239], YAK [212, 239], ARC-FE [40], N-FE [94, 140], S-FE [76, 77, 109, 212]

- japonicum Lindb. - RUS-AS: N-FE [140], S-FE [76, 212]

Cnestrum I.Hagen [Rhabdoweisiaceae]

- alpestre (Wahlenb. ex Huebener) Nyholm ex Mogensen (Cynodontium alpestre (Wahlenb. ex Huebener) Milde) - RUS-EUR: ARC [47, 127, 150], NW [273, 364, 407], N-UR [150, 415], S-UR [150], RUS-AS: ARC-WSIB [136], ARC-ESIB [164, 275], S-SIB [69, 197, 212], E-SIB [126, 212], ARCYAK [47, 239], YAK [239], ARC-FE [40, 212], N-FE [40], S-FE [212, 275, 221]

— glaucescens (Lindb. \& Arnell) Holmen ex Mogensen \& Steere (Cynodontium glaucescens (Lindb. \& Arnell) Paris - RUS-EUR: NW [145], RUS-AS: ARC-ESIB [275], S-SIB [69, 70], E-SIB [69, 212, 275], ARC-YAK [47, 239], YAK [239], ARC-FE [40], N-FE [40], S-FE [76, 212] - $\{48\}$

- schisti (F.Weber \& D.Mohr) I.Hagen - UKR: C [66], RUS-EUR: ARC [47, 150], NW [212, 364, 407], NE [207, 415], N-UR [150, 207, 212], S-UR [150, 207, 212], CAUC [231], RUS-AS: S-SIB [69, 70, 212], E-SIB [74, 126, 212], YAK [239], ARC-FE [40], N-FE [40, 94, 140], S-FE [76, 212, 221]

Codriophorus P.Beauv. [Grimmiaceae]

- acicularis (Hedw.) P.Beauv. (Racomitrium aciculare (Hedw.) Brid.) - EST [237], UKR: C [66, 81], M [66], RUS-EUR: ARC [47, 81], NW [81, 212, 273, 364, 407], N-UR [81, 150, 212], S-UR [81, 150, 207, 212], CAUC [81, 231], GEO [81, 117], ARM [297] - \{49\}

- aquaticus (Brid. ex Schrad.) Bednarek-Ochyra \& Ochyra (Racomitrium aquaticum (Brid. ex Schrad.) Brid.) - UKR: C [66, 81], RUS-EUR: NW [81, 407], S-UR [81, 207], CAUC [55, 81, 212, 231], GEO $[81,117]-\{50\}$

- brevisetus (Lindb.) Bednarek-Ochyra \& Ochyra (Racomitrium brevisetum Lindb.) - RUS-AS: N-FE [81, 140], S-FE [81, 109, 212]

- carinatus (Cardot) Bednarek-Ochyra \& Ochyra - RUS-AS: N-FE [81, 140], S-FE [116]

- corrugatus Bednarek-Ochyra - RUS-AS: ARC-YAK [81, 239], S-SIB [81], E-SIB [388], N-FE [81, 140], S-FE [67]

- fascicularis (Hedw.) Bednarek-Ochyra \& Ochyra (Racomitrium fasciculare (Hedw.) Brid.) - EST [237], RUS-EUR: ARC [47, 81, 150], NW [81, 212, 273, 364, 407], KLN [309], N-UR [81, 150, 415], RUSAS: ARC-ESIB [47, 81], ARC-FE [40, 81, 212], N-FE [81, 94, 140], S-FE [76, 77, 81, 212]

- mollis (Cardot) Bednarek-Ochyra \& Ochyra - RUS-AS: N-FE [81]

Conardia H.Rob. [Calliergonaceae]

- compacta (Drumm. ex Müll.Hal.) H.Rob. (Amblystegium compactum (Drumm. ex Müll.Hal.) Austin, Rhynchostegiella compacta (Drumm. ex Müll.Hal.) Loeske) - LAT [3], EST [237], UKR: M [66], RUS-EUR: NW [407], C [207, 345], S-UR [150, 207], CAUC [212, 231], KAZ [20, 296], TAD [294, 296], KYR [296], RUS-AS: W-SIB [266], S-SIB [69, 70, 197], E-SIB [162], YAK [239], S-FE [77] 
Conostomum Sw. ex F.Weber \& D.Mohr [Bartramiaceae]

- tetragonum (Hedw.) Lindb. - RUS-EUR: ARC [47, 127, 150], NW [212, 273, 364, 407], N-UR [150, 415], RUS-AS: ARC-WSIB [136], ARC-ESIB [164, 212, 275], S-SIB [69, 70, 212], E-SIB [69, 212], ARC-YAK [47, 212, 239], YAK [239], ARC-FE [40, 212], N-FE [94, 140], S-FE [212, 221]

Coscinodon Spreng. [Grimmiaceae] $\{51\}$

- cribrosus (Hedw.) Spruce - UKR: C [66], K [328], RUS-EUR: ARC [47, 127, 150], NW [364, 407], CAUC [102, 212, 231, 248], GEO [117], KAZ [296, 356], TAD [294, 295], KYR [296], RUS-AS: SSIB [69, 70, 212], E-SIB [74, 212], ARC-YAK [47, 239], YAK [239], ARC-FE [40], N-FE [94, 140], S-FE [79, 212]

Cratoneuron (Sull.) Spruce [Amblystegiaceae], see also Palustriella commutata, P. decipiens

- curvicaule (Jur.) G.Roth (Callialaria curvicaule (Jur.) Ochyra, Cratoneuron filicinum var. curvicaule (Jur.) Mönk.) - RUS-EUR: ARC [47], NE [415], KAZ [296, 356], TAD [296], RUS-AS: ARC-ESIB [164], E-SIB [163], W-SIB [265], ARC-YAK [47, 239], YAK [212, 239], ARC-FE [40, 212]

- filicinum (Hedw.) Spruce - LTV [244], LAT [3], EST [237], BEL [355], UKR: C [66], M [66], K [66, 328], MLD [367], RUS-EUR: ARC [47, 150], NW [207, 212, 364, 407], NE [207, 212, 415], KLN [309], C [207, 212], N-UR [150, 207, 212], S-UR [150, 207, 212], SE [207], CAUC [55, 208, 212, 231, 248], GEO [117], ARM [297], AZE [277], KAZ [156, 296, 356], TUR [267, 296], UZB [347], TAD [294, 296], KYR [296, 349, 351], RUS-AS: ARC-ESIB [93, 212], W-SIB [265], S-SIB [69, 70, 212], E-SIB [69, 212, 275], ARC-YAK [47, 212, 239], YAK [212, 239], ARC-FE [40, 212], N-FE [140], S-FE [76, 77, 109, 212]

Crossidium Jur. [Pottiaceae]

- crassinerve (De Not.) Jur. (C. chloronotos auct. non (Brid.) Limpr.) - MLD [367], AZE [277], TUR [296], TAD [294, 295]

- squamiferum (Viv.) Jur. - UKR: K [328], RUS-EUR: C [207], SE [207], CAUC [232], GEO [117], ARM [297], AZE [277], KAZ [160, 295], TUR [295, 296], UZB [296], TAD [295], KYR [296, 351], RUS-AS: S-SIB [70, 197]

- squamiferum var. pottioideum (De Not.) Mönk. (C. griseum (Jur.) Jur.) - GEO [102], TUR [267, 295, 296], UZB [295, 296], TAD [294, 295], KYR [267, 296]

Crumia W.B.Schofield [Pottiaceae]

- latifolia (Kindb.) W.B.Schofield - ARM [297]

Cryphaea D.Mohr [Cryphaeaceae]

- amurensis Ignatov - RUS-AS: S-FE [116, 203, 212, 221]

- heteromalla (Hedw.) D.Mohr - RUS-EUR: CAUC [203]

Ctenidium (Schimp.) Mitt. [Hylocomiaceae], see also Stereodon procerrimus

- ?capillifolium (Mitt.) Broth. - RUS-AS: S-FE [376] - \{52\}

- molluscum (Hedw.) Mitt. - LTV [244], LAT [3], EST [237], BEL [355], UKR: C [66], M [66], K [66, 328], RUS-EUR: NW [212, 407], KLN [309], CAUC [55, 208, 212, 231, 248], GEO [117], ARM [297], AZE [277], RUS-AS: ARC-WSIB [136], ARC-ESIB [92, 96, 212], E-SIB [163], ARCYAK [20, 47, 239], YAK [239], ARC-FE [40, 212], N-FE [?140], ?S-FE [37, 212] - $\{53\}$

Cynodontium Bruch et al. [Rhabdoweisiaceae], see also Cnestrum alpestre, C. glaucescens

— asperifolium (Lindb. \& Arnell) Paris - RUS-EUR: NW [207], NE [415], N-UR [207, 212], S-UR [150, 207, 212], KAZ [296, 356], KYR [296], RUS-AS: S-SIB [69, 70, 212], E-SIB [74, 212, 388], ARC-YAK [239], YAK [239], N-FE [140], S-FE [76, 212]

- bruntonii (Sm.) Bruch et al. (Oreoweisia bruntonii (Sm.) Milde) - RUS-EUR: N-UR [150, 415], UKR: C [66], K [328], KAZ [296, 356]

- fallax Limpr. - RUS-EUR: ?ARC [47, 150], ?NE [415], ?N-UR [150], ?S-UR [150], CAUC [55, 212, 231, 248], ?RUS-AS: S-SIB [70], ARC-YAK [47] $-\{54\}$

- gracilescens (F.Weber \& D.Mohr) Schimp. - UKR: C [66], RUS-EUR: NE [415], CAUC [102, 212, 231], GEO [117], RUS-AS: ARC-ESIB [212]

- polycarpon (Hedw.) Schimp. - UKR: C [66], M [66], RUS-EUR: NE [212, 416, 417], S-UR [150], CAUC [248], GEO [117], AZE [277], RUS-AS: S-SIB [70, 212], E-SIB [74, 212], ARC-YAK [47], ARC-FE [40], N-FE [40], S-FE [76, 212] 
- strumiferum (Hedw.) Lindb. - LAT [3], EST [237], UKR: C [66], RUS-EUR: ARC [47, 127, 150], NW [207, 212, 364, 407], NE [207, 212, 415], KLN [309], C [207], N-UR [150, 207, 212], S-UR [150, 207, 212], CAUC [55, 212, 231, 248], KAZ [296, 356], RUS-AS: ARC-WSIB [136], ARC-ESIB [164, 212, 275], S-SIB [69, 70, 212], E-SIB [69, 212, 275], ARC-YAK [47, 239], YAK [239], ARC-FE [40], N-FE [94, 140], S-FE [76, 212, 221]

- suecicum (Arnell \& C.E.O.Jensen) I.Hagen - RUS-EUR: NW [364], N-UR [150, 415]

- tenellum (Schimp.) Limpr. - LAT [3], RUS-EUR: ARC [47, 127, 150, 415], NW [212, 273, 364, 407], NE [53, 207, 212, 415], N-UR [150, 207, 212, 415], S-UR [150, 207, 212], GEO [117], KAZ [296, 356], RUS-AS: ARC-WSIB [136], ARC-ESIB [164, 212, 275], W-SIB [265], S-SIB [69, 70, 212], E-SIB [69, 212, 275], ARC-YAK [47, 239], YAK [239], ARC-FE [40], N-FE [94, 140], S-FE $[76,212,221]$

Cyrtomnium Holmen [Mniaceae]

- hymenophylloides (Huebener) T.J.Kop. - RUS-EUR: ARC [47, 150, 415], NW [364, 407], NE [207, 212, 364, 415], N-UR [150, 207, 212, 415], S-UR [150, 207, 212], GEO [13], RUS-AS: ARC-WSIB [136], ARC-ESIB [164, 212], S-SIB [69, 70, 212], E-SIB [69, 212], ARC-YAK [47, 239], YAK [212, 239], ARC-FE [40], N-FE [94], S-FE [212, 221]

- hymenophyllum (Bruch et al.) Holmen - RUS-EUR: ARC [47, 127, 150], NW [364], N-UR [150], RUS-AS: ARC-WSIB [136], ARC-ESIB [164, 212], S-SIB [69, 70, 212], E-SIB [74, 212], ARC-YAK [47], YAK [?239], ARC-FE [40, 212], N-FE [140], S-FE [67]

Desmatodon see Tortula spp., Hennediella heimii

Dichelyma Myrin [Fontinalaceae]

— capillaceum (Dicks.) Myrin - EST [237], RUS-EUR: NW [137], RUS-AS: W-SIB [137], E-SIB [167], ARC-FE [43]

- falcatum (Hedw.) Myrin - LTV [244], EST [237], BEL [355], UKR: C [66], RUS-EUR: ARC [47, 150, 415], NW [207, 212, 364, 407], NE [207, 212, 415], C [207, 212], N-UR [150, 207, 212], S-UR [150, 207, 212], KAZ [296], RUS-AS: ARC-WSIB [136], W-SIB [212, 265], S-SIB [69, 70, 212], ESIB [212, 275], YAK [239], ARC-FE [40], N-FE [40, 140]

Dichodontium Schimp. [Rhabdoweisiaceae]

- flavescens (Dicks.) Lindb. (D. pellucidum var. flavescens (Dicks.) Moore) - UKR: C [66], RUS-EUR: NW [407] - $\{55\}$

— palustre (Dicks.) M.Stech (Anisothecium palustre (Dicks.) I.Hagen, Dicranella palustris (Dicks.) Crundw., Diobelonella palustris (Dicks.) Ochyra) - UKR: C [66], RUS-EUR: NW [100, 212, 273, 364], CAUC [55, 212, 231], GEO [117], RUS-AS: ARC-FE [40], N-FE [140], S-FE [77, 212] - \{56\}

- pellucidum (Hedw.) Schimp. - LTV [244], LAT [3], EST [237], BEL [355], UKR: C [66], RUSEUR: ARC [47, 127, 150], NW [87, 207, 364, 407], NE [207, 212, 415], C [207, 212, 345], N-UR [150, 207, 212], S-UR [150, 207, 212], CAUC [55, 212, 231, 248], GEO [117], ARM [297], AZE [277], RUS-AS: ARC-ESIB [164], W-SIB [307], S-SIB [70, 212], E-SIB [126, 212], ARC-YAK [47, 239], YAK [239], ARC-FE [40, 212], N-FE [40, 140], S-FE [76, 77, 109, 212]

Dicranella (Müll.Hal.) Schimp. [Dicranaceae], see also Dichodontium palustre

- cerviculata (Hedw.) Schimp. - LTV [244], LAT [3], EST [237], BEL [355], UKR: C [66], M [66], RUS-EUR: ARC [47, 127, 150], NW [207, 212, 364, 407], NE [207, 212, 415], KLN [309], C [207, 212], N-UR [150, 207], S-UR [150, 207, 212], SE [207], RUS-AS: ARC-WSIB [136], ARC-ESIB [164, 275], W-SIB [212, 265], S-SIB [69, 70, 212], E-SIB [212, 275], ARC-YAK [47, 212, 239], YAK [239], ARC-FE [40], N-FE [94, 140], S-FE [77, 109, 212]

- crispa (Hedw.) Schimp. (Anisothecium vaginale (Dicks. ex With.) Loeske) - LTV [244], LAT [3], EST [237], BEL [355], UKR: M [66], RUS-EUR: ARC [47, 127, 150, 415], NW [207, 364, 407], NE [207, 212, 415], KLN [309], C [207, 212], N-UR [90, 150, 207, 212], S-UR [150], SE [207], RUS-AS: ARC-WSIB [136], ARC-ESIB [164, 212, 275], W-SIB [212, 265], S-SIB [69, 197], ESIB [70, 163, 212, 275], ARC-YAK [47, 239], YAK [212, 239], ARC-FE [40, 212], N-FE [40, 140], S-FE $[212,221]$

- curvipes (Lindb.) Ignatov, comb. nov. - Dicranella heteromalla var. curvipes Lindb., Contributio ad Floram Cryptogamam Asiae Boreali-Orientalis 243. 1872. - RUS-AS: S-FE [361] $-\{57\}$ 
- grevilleana (Brid.) Schimp. (Anisothecium grevilleanum (Brid.) Lindb.) - RUS-EUR: ARC [47, 150, 415], NW [364, 407], NE [207, 212, 415], C [207], N-UR [150, 207, 212], S-UR [150], GEO [117], RUS-AS: ARC-WSIB [136], ARC-ESIB [164], E-SIB [126, 212], YAK [212, 239], ARC-FE [40, 212], N-FE [94, 95]

- heteromalla (Hedw.) Schimp. (D. caucasica (Müll.Hal.) Broth.) - LTV [244], LAT [3], EST [237], BEL [355], UKR: C [66], M [66], RUS-EUR: ARC [150], NW [207, 212, 364, 407], NE [207, 212, 415], KLN [309], C [207, 212], N-UR [150, 207, 212], S-UR [150, 207, 212], SE [207, 212], CAUC [55, 212, 231], GEO [117], ARM [297], AZE [277], KAZ [156, 157], KYR [296], RUS-AS: W-SIB [265], S-SIB [69, 70, 212], E-SIB [74, 212, 247], YAK [239], N-FE [140], S-FE [76, 77, 109, 212]

- howei Renauld \& Cardot - TUR [11, 296]

- humilis R.Ruthe (Anisothecium humile (R.Ruthe) Lindb., A. rigidulum (Hedw.) C.E.O.Jensen) - EST [397], RUS-EUR: NW [207, 407], NE [207, 212, 415], C [207, 212], N-UR [90, 207], S-UR [207], RUS-AS: ARC-WSIB [136], ARC-ESIB [164, 212], W-SIB [264], E-SIB [212, 275], YAK [239], SFE [76, 77, 212]

- rufescens (Dicks.) Schimp. (Anisothecium rufescens (Dicks.) Lindb.) - LTV [244], EST [237], BEL [355], UKR: C [66], M [66], K [66, 328], RUS-EUR: NW [207, 291, 364], NE [207], KLN [309], C [207, 212], GEO [117], RUS-AS: ARC-WSIB [136], S-SIB [70, 212], S-FE [76, 77, 109]

- schreberiana (Hedw.) Hilf. ex H.A.Crum \& L.E.Anderson (Anisothecium schreberianum (Hedw.) Dixon) - LTV [244], LAT [3], EST [237], BEL [355], UKR: C [66], M [66], RUS-EUR: ARC [47, 127, 150], NW [87, 207, 364, 407], NE [207, 212, 415], KLN [309], C [207, 212], N-UR [90, 207], S-UR [150, 207, 212], SE [207], CAUC [55, 212, 248], RUS-AS: ARC-WSIB [136], ARC-ESIB [164], WSIB [265], S-SIB [69, 197, 212], E-SIB [212, 247], ARC-YAK [47, 212, 239], YAK [239], ARC-FE [40, 212], N-FE [140], S-FE [76, 212, 221]

- staphylina H.Whitehouse (Anisothecium staphylinum (H.Whitehouse) Sipman, Rubers \& Riemann) LTV [244], RUS-EUR: KLN [309]

- subsecunda Besch. - RUS-AS: S-FE [361]

- subulata (Hedw.) Schimp. - LTV [244], LAT [3], EST [237], BEL [355], UKR: C [66], RUSEUR: ARC [47, 127, 150], NW [207, 212, 364, 407], NE [207, 212, 415], C [207, 212], N-UR [150, 207, 212], S-UR [150, 207, 212], CAUC [55, 212, 231, 248], GEO [117], KAZ [159, 157], KYR [296], RUS-AS: ARC-WSIB [136], ARC-ESIB [164, 212, 275], W-SIB [265], S-SIB [69, 70, 212], E-SIB [212, 275], ARC-YAK [47, 212, 239], YAK [239], ARC-FE [40, 212], N-FE [140, 237], S-FE $[77,109,212]$

- varia (Hedw.) Schimp. (Anisothecium varium (Hedw.) Mitt.) - LTV [244], LAT [3], EST [237], BEL [355], UKR: C [66], M [66], K [66, 328], MLD [367], RUS-EUR: NW [207, 364, 407], NE [207, 212 , 415], KLN [309], C [207, 212], N-UR [90, 150, 207], S-UR [150, 207, 212], SE [207], CAUC [208, 212, 248], GEO [117], ARM [297], AZE [277], KAZ [157], TUR [11], TAD [294, 295], KYR [296], UZB [267], RUS-AS: ARC-WSIB [136], ARC-ESIB [164], W-SIB [265], S-SIB [69, 70, 212], ARCYAK [47], YAK [239], N-FE [140], S-FE [76, 212, 221]

Dicranodontium Bruch et al. [Leucobryaceae]

— asperulum (Mitt.) Broth. - BEL [355], UKR: C [66], RUS-AS: S-FE [212, 221]

- denudatum (Brid.) E.Britton - LTV [244], LAT [3], BEL [355], UKR: C [66], M [66], K [66, 328], RUS-EUR: NW [212, 407], NE [207, 415], KLN [309], C [207], N-UR [150, 207, 212], S-UR [150, 212], CAUC [55, 212, 231, 248], GEO [117], ARM [297], RUS-AS: S-SIB [69, 70, 212], E-SIB [74, 212], YAK [239], ARC-FE [40], N-FE [140], S-FE [76, 77, 109, 212]

Dicranoloma (Renauld) Renauld [Dicranaceae]

- cylindrothecium (Mitt.) Sakurai - RUS-AS: S-FE [116]

Dicranoweisia Milde [Rhabdoweisiaceae], see also Hymenoloma crispulum, H. intermedium

- cirrata (Hedw.) Lindb. - LAT [4], BEL [355], UKR: C [66], M [66], K [328], RUS-EUR: KLN [309], CAUC [232], GEO [102, 117], ARM [297], AZE [277], KAZ [156, 159, 393] - \{58\}

Dicranum Hedw. [Dicranaceae]

- acutifolium (Lindb. \& Arnell) C.E.O.Jensen - RUS-EUR: ARC [47, 127, 150, 415], NW [87, 212, 364, 407], NE [207, 415], N-UR [150, 415], S-UR [150, 207], RUS-AS: ARC-WSIB [136], ARC- 
ESIB [164, 212], S-SIB [69, 70, 212], E-SIB [74, 212], ARC-YAK [47, 212, 239], YAK [212, 239], ARC-FE [40, 212], N-FE [140], S-FE [212, 221]

- angustum Lindb. - RUS-EUR: ARC [47, 127, 150, 415], NW [212, 224, 364, 407], NE [212, 415], NUR [150, 224, 415], RUS-AS: ARC-WSIB [136, 224], ARC-ESIB [212, 275], W-SIB [224], S-SIB [212, 224], E-SIB [212, 224, 275], ARC-YAK [47, 212], YAK [212, 224, 239], N-FE [94, 224], S-FE $[212,221]$

- bonjeanii De Not. - LTV [244], LAT [3], EST [237], BEL [355], UKR: C [66], M [66], RUS-EUR: ARC [47, 127, 150], NW [207, 212, 364, 407], NE [207, 212, 415], KLN [309], C [207, 212], N-UR [150, 207, 212], S-UR [150, 207, 212], SE [207], CAUC [55, 212, 231, 248], GEO [117], AZE [277], KAZ [296, 356], TAD [295], RUS-AS: ARC-WSIB [136], ARC-ESIB [?47], W-SIB [212, 265, 275], S-SIB [69, 70, 212], E-SIB [69, 212, 275], ARC-YAK [47, 239], YAK [212, 239], ARC-FE [40, 212], N-FE [94, 140], S-FE [109, 212, 221]

- brevifolium (Lindb.) Lindb. (D. muehlenbeckii var. brevifolium Lindb.) - EST [237], RUS-EUR: ARC [47, 127, 150], NW [87, 212, 364, 407], NE [207, 212, 415], C [135, 207, 212], N-UR [150, 415], S-UR [150, 207, 212], CAUC [102, 212, 231], ARM [297], KYR [296], RUS-AS: ARC-WSIB [136], ARC-ESIB [164, 212], S-SIB [197, 212], E-SIB [212, 275], ARC-YAK [47, 212, 239], YAK [239], ARC-FE [40], N-FE [40, 140], S-FE [212, 221]

- dispersum Engelmark (D. orientale Otnyukova) - RUS-EUR: S-UR [207, 326], CAUC [212, 248, 326], RUS-AS: S-SIB [212, 326], S-FE [326] - \{59)

- drummondii Müll.Hal. (D. elatum Lindb., D. robustum Bruch et al.) - LTV [244], LAT [3], EST [237], RUS-EUR: NW [212, 273, 364, 407], NE [207, 212, 415], N-UR [150, 207, 212], S-UR [207], RUS-AS: S-SIB [69, 70, 212], YAK [239], S-FE [109]

- elongatum Schleich. ex Schwägr. (?D. tundrae Lindb. \& Arnell, ?D. atratum Geh.) - UKR: C [66], RUS-EUR: ARC [47, 127, 150], NW [212, 273, 224, 364, 407], NE [207, 212, 224, 415], N-UR [150, 212, 224], S-UR [150], CAUC [212, 248], GEO [117], KAZ [296, 356], KYR [296], RUS-AS: ARCWSIB [136, 224, 275], ARC-ESIB [164, 212, 224, 275], W-SIB [224], S-SIB [69, 70, 212, 224], ESIB [69, 212, 224, 275], ARC-YAK [47, 212, 224, 239], YAK [212, 224, 239], ARC-FE [40, 212, 224], N-FE [94, 140, 224], S-FE [212, 221, 224] - $\{60\}$

- flagellare Hedw. (Orthodicranum flagellare (Hedw.) Loeske) - LTV [244], LAT [3], EST [237], BEL [355], UKR: C [66], M [66], RUS-EUR: NW [207, 212, 407], NE [207, 212, 415], KLN [309], C [207, 212], N-UR [90, 150, 207], S-UR [150, 207, 212], SE [207], CAUC [231], GEO [117], RUSAS: W-SIB [212, 265, 275], S-SIB [69, 70, 212], E-SIB [69, 212, 275], YAK [239], N-FE [140], S-FE $[76,77,109,212]$

- flexicaule Brid. (D. congestum Brid., D. fuscescens var. congestum (Brid.) Kindb., D. fuscescens var. flexicaule (Brid.) Wilson) - LTV [244], LAT [3], EST [237], UKR: C [66], M [66], RUS-EUR: ARC [47, 127, 150], NW [207, 212, 364, 407], NE [207, 212, 415], N-UR [150, 207, 212], S-UR [150, 207, 212], CAUC [55, 212, 231], GEO [117], ARM [297], AZE [277], KAZ [296], KYR [351], RUS-AS: ARC-WSIB [136], ARC-ESIB [164, 212, 275], W-SIB [212, 265], S-SIB [69, 70, 212], E-SIB [69, 212, 275], ARC-YAK [47, 212, 239], YAK [212, 239], ARC-FE [40, 212], N-FE [94, 140], S-FE [76, $109,212]$

- fragilifolium Lindb. - RUS-EUR: ARC [47, 150], NW [87, 212, 364, 407], NE [207, 212, 415], C [207, 212, 345], N-UR [150, 207, 212], S-UR [150, 207, 212], KAZ [296, 356], RUS-AS: ARCWSIB [275], ARC-ESIB [275], W-SIB [212, 265], S-SIB [69, 70, 212], E-SIB [69, 212, 275], ARCYAK [239], YAK [212, 239], N-FE [94, 140], S-FE [76, 77, 109, 212]

- fulvum Hook. - UKR: C [66], M [66], RUS-EUR: KLN [309], CAUC [248], GEO [31], RUS-AS: SFE $[212,268]$

- fuscescens Turner - LTV [244], LAT [3], EST [237], BEL [355], UKR: C [66], M [66], RUS-EUR: ARC [47, 150], NW [207, 212, 364, 407], NE [207, 212, 415], KLN [309], C [207, 212], N-UR [150, 207, 212], S-UR [150, 207, 212], SE [207], CAUC [55, 212, 248], GEO [117], ARM [297], AZE [277], KAZ [159, 296], KYR [296], RUS-AS: ARC-WSIB [136], ARC-ESIB [47, 96, 212], W-SIB [212, 265], S-SIB [69, 70, 212], E-SIB [69, 212], ARC-YAK [47, 239], YAK [212, 239], ARC-FE [40], N-FE [40, 140], S-FE [76, 77, 109, 212] 
- groenlandicum Brid. - UKR: C [66], RUS-EUR: ARC [47, 150, 224, 415], NW [212, 224, 291, 364], N-UR [150], RUS-AS: ARC-WSIB [136, 224], ARC-ESIB [164, 212, 224], S-SIB [70, 212], E-SIB [126, 212], ARC-YAK [47, 212, 224, 239], YAK [212, 224, 239], ARC-FE [40, 224], N-FE [94, 140, 224], S-FE [109, 224]

- hamulosum Mitt. - ?KAZ [296, 356], RUS-AS: N-FE [140], S-FE [109] - \{61\}

- japonicum Mitt. - RUS-AS: N-FE [140], S-FE [109]

- laevidens R.S.Williams - RUS-EUR: ARC [224], NW [224], NE [224], RUS-AS: ARC-WSIB [224], ARC-ESIB [164, 224], E-SIB [163], ARC-YAK [224, 239], YAK [224, 239], ARC-FE [40, 224], NFE [40, 140], S-FE [224]

— leioneuron Kindb. - LTV [244], LAT [3], EST [237], RUS-EUR: NW [364], NE [207, 415], C [207], KAZ [70], RUS-AS: ARC-WSIB [136], ARC-ESIB [39], W-SIB [265], S-SIB [70], YAK [239], ARCFE [40, 212], N-FE [40, 140], S-FE [76]

- majus Turner - LTV [244], LAT [3], EST [237], BEL [355], UKR: C [66], K [66, 328], RUS-EUR: ARC [47, 127, 150], NW [207, 212, 364, 407], NE [207, 212, 415], KLN [309], C [207, 212], N-UR [150, 207, 212], S-UR [150, 207, 212], CAUC [14], GEO [117], AZE [277], RUS-AS: ARC-WSIB [136], ARC-ESIB [212, 275], W-SIB [265], S-SIB [69, 70, 212], E-SIB [212, 275], ARC-YAK [47, 239], YAK [212, 239], ARC-FE [40, 212], N-FE [40, 140], S-FE [76, 77, 109, 212]

- mayrii Broth. (Orthodicranum mayrii (Broth.) Smirnova) - RUS-AS: S-FE [76, 212]

- montanum Hedw. (Orthodicranum montanum (Hedw.) Loeske) - LTV [244], LAT [3], EST [237], BEL [355], UKR: C [66], M [66], RUS-EUR: ARC [47, 150], NW [207, 212, 364, 407], NE [207, 212, 415], KLN [309], C [207, 212], N-UR [150, 207, 212], S-UR [150, 207, 212], SE [207, 212], CAUC [55, 208, 212, 231, 248], GEO [117], ARM [297], AZE [277], KAZ [156, 159, 157],KYR [267, 296], UZB [267], TAD [267], RUS-AS: W-SIB [212, 265, 275], S-SIB [69, 70, 212], E-SIB [69, 212, 275], YAK [239], N-FE [94, 140], S-FE [76, 212, 221]

- muehlenbeckii Bruch et al. - EST [236], UKR: C [66], K [66, 328], RUS-EUR: ARC [47, 150], NW [145, 207, 212], NE [207, 212, 415], N-UR [150, 207, 415], S-UR [150, 207, 212, 224], CAUC [55, 212, 224, 231, 248], GEO [117], ARM [297], AZE [277], KAZ [296], KYR [296], RUS-AS: ARCESIB [212], W-SIB [224, 265], S-SIB [69, 70, 212, 224], E-SIB [212, 247], ARC-YAK [47, 212, ?239], YAK [224, 239], ARC-FE [40], N-FE [94, 140], S-FE [212, 268] - \{62\}

- nipponense Besch. - RUS-AS: S-FE [114, 322]

- polysetum Sw. (D. rugosum Brid., D. undulatum Ehrh. ex F.Weber \& D.Mohr, nom. illeg.) - LTV [244], LAT [3], EST [237], BEL [355], UKR: C [66], M [66], MLD [367], RUS-EUR: ARC [150], NW [207, 212, 364, 407], NE [207, 415], KLN [309], C [207, 212], N-UR [150, 207, 212], S-UR [150, 207, 212], SE [207, 212], CAUC [18, 55, 212], GEO [117], ARM [297], AZE [277], KAZ [156, 296, 356], TAD [295], RUS-AS: ARC-WSIB [136], W-SIB [212, 265, 275], S-SIB [69, 70, 212], E-SIB $[69,212,275]$, YAK $[212,239]$, N-FE [94, 140], S-FE [76, 109, 212]

- scoparium Hedw. - LTV [244], LAT [3], EST [237], BEL [355], UKR: C [66], M [66], K [66, 328], MLD [367], RUS-EUR: ARC [47, 150], NW [207, 212, 364, 407], NE [207, 212, 415], KLN [309], C [207, 212], N-UR [150, 207, 212], S-UR [150, 207, 212], SE [207, 212], CAUC [55, 208, 212, 231, 248], GEO [117], ARM [297], AZE [277], KAZ [156, 159, 296, 356], KYR [296, 350], RUS-AS: ARC-WSIB [?47], ARC-ESIB [?47, 275], W-SIB [212, 265], S-SIB [69, 70, 212], E-SIB [69, 212, 275], ?ARC-YAK [?47, 239], YAK [212, 239], ARC-FE [40], N-FE [94, 140], S-FE [76, 77, 109, 212]

- spadiceum J.E.Zetterst. (D. neglectum Jur. ex De Not., ?D. spadiceum J.E.Zetterst. var. subscabrifolium Schljakov) - RUS-EUR: ARC [47, 127, 150, 224], NW [212, 224, 273, 364, 407], NE [207, 212 , 224, 415], N-UR [150, 207, 212, 224], S-UR [150, 207, 212, 224], CAUC [55, 212, 224, 231, 248], GEO [117], ARM [297], AZE [277], KAZ [159, 296], KYR [296, 351], RUS-AS: ARC-WSIB [136, 224], ARC-ESIB [164, 212, 224], W-SIB [212, 224], S-SIB [69, 70, 212, 224, 265], E-SIB [69, 212 , 224, 275], ARC-YAK [47, 212, 224], YAK [212, 224, 239], ARC-FE [40, 212, 224], N-FE [94, 140, 224], S-FE [212, 221, 224] - \{63)

- spurium Hedw. - LTV [244], LAT [3], EST [237], BEL [355], UKR: C [66], M [66], RUS-EUR: NW [207, 212, 364, 407], NE [207, 415], KLN [309], C [207], RUS-AS: N-FE [?140] — \{64\}

- tauricum Sapjegin (D. strictum Schleich. ex D.Mohr, nom. illeg., Orthodicranum tauricum (Sapjegin) 
Smirnova) - BEL [355], UKR: C [66], M [66], K [66, 328], RUS-EUR: KLN [309], C [207, 212, 345], SE [207], CAUC [55, 212, 231, 248], GEO [117], AZE [277], RUS-AS: N-FE [140] — \{\}

- undulatum Schrad. ex Brid. (D. bergeri Blandow, D. affine Funck) - LTV [244], LAT [3], EST [237], BEL [355], UKR: C [66], RUS-EUR: ARC [47, 127, 150], NW [207, 212, 364, 407], NE [207, 212, 415], KLN [309], C [207, 212], N-UR [90, 150, 207, 212], S-UR [150, 207, 212], CAUC [212, 231, 248], ARM [297], RUS-AS: ARC-WSIB (?47), ARC-ESIB (?47, 275), W-SIB [212, 265, 275], S-SIB [69, 70, 212], E-SIB [69, 212, 275], ARC-YAK [47, 239], YAK [212, 239], ARC-FE [40], N-FE [94, 140], S-FE [109, 212, 221]

— viride (Sull. \& Lesq.) Lindb. - LTV [244], LAT [3], EST [237], BEL [355], UKR: C [66], M [66], RUS-EUR: NW [207], NE [207, 415], KLN [309], C [207, 212, 345], N-UR [207], S-UR [150, 207, 212], CAUC [55, 208, 212], GEO [117], RUS-AS: S-FE [76, 77, 109, 212] - \{65\}

Didymodon Hedw. [Pottiaceae]

- acutus (Brid.) K.Saito (Barbula acuta (Brid.) Brid.) - BEL [355], UKR: C [66], M [66], K [66, 328], MLD [367], RUS-EUR: KLN [309], C [?212, ?345], S-UR [150], CAUC [55, 212, 243], GEO [117, 243], ARM [297], AZE [243, 277], KAZ [243], UZB [243], TAD [243, 295], KYR [243], RUS-AS: ARC-FE $[40]-\{66\}$

— anserinocapitatus (X.J.Li) R.H.Zander - KAZ [243], RUS-AS: S-SIB [323, 327], YAK [239]

- asperifolius (Mitt.) H.A.Crum, Steere \& L.E.Anderson (Barbula asperifolia Mitt.) - RUS-EUR: ARC [47, 150], CAUC [243], KAZ [157, 243, 296, 356], RUS-AS: ARC-ESIB [96, 212], S-SIB [70, 212], ARC-YAK [47, 239], YAK [212, 239], ARC-FE [40, 212], N-FE [140]

- asperifolius var. gorodkovii (Abramova \& I. I. Abramov) Afonina (D. gorodkovii (Abramova \& I. I. Abramov) Schljakov) - RUS-AS: ARC-ESIB [164], E-SIB [163], ARC-YAK [239], ARC-FE [40] — \{67\}

- australasiae (Hook. \& Grev.) R.H.Zander (D. aaronis (Lorentz) J.Guerra, D. incrassatus (Lindb.) Broth., Trichostomopsis aaronis (Lorentz) S.Agnew \& C.C.Towns., T. australasiae (Hook. \& Grev.) H.Rob.) - GEO [117], KAZ [243], TUR [8, 296, 368], UZB [243] - \{68\}

- cordatus Jur. (Barbula cordata (Jur.) Loeske) - UKR: K [66, 328], MLD [367], RUS-EUR: CAUC [102, 243, 248], GEO [117], ARM [297], TAD [295], RUS-AS: S-FE [76]

- fallax (Hedw.) R.H.Zander (Barbula fallax Hedw.) - LTV [244], LAT [3], EST [237], BEL [355], UKR: C [66], M [66], K [66, 328], MLD [367], RUS-EUR: NW [149, 207, 407], NE [207, 212, 415], KLN [309], C [207, 212], N-UR [90, 150, 207, 212], S-UR [150, 207, 212], SE [207], CAUC [55, 208, 212, 231, 243], GEO [117, 243], ARM [297], AZE [277], KAZ [243, 296, 356], TUR [267, 295, 368], UZB [296], TAD [294, 295], KYR [296], RUS-AS: ARC-ESIB [164], W-SIB [265], S-SIB [69, 70, 212], E-SIB [163, 275], ARC-YAK [47, 239], YAK [239]

- ferrugineus (Schimp. ex Besch.) M.O.Hill (Barbula reflexa (Brid.) Brid.) - LTV [244], EST [237], UKR: C [66], M [66], RUS-EUR: NW [407], NE [207, 212, 415], C [207, 212], N-UR [90, 91, 207], S-UR [91, 150, 207], SE [?207], CAUC [212, 231, 243, 248], GEO [117, 243], ARM [297], AZE [277], KAZ [243], TUR [295], TAD [294, 295], KYR [267, 296], RUS-AS: ARC-ESIB [?47, 93], WSIB [307], S-SIB [69, 70], E-SIB [163], ARC-YAK [47, 239], YAK [239], ARC-FE [40, 212], N-FE [40], S-FE [76, 212, 221]

- gaochienii B.C.Tan \& Y.Jia - RUS-AS: S-SIB [212, 323], N-FE [140]

- giganteus (Funck) Jur. (Barbula gigantea Funck, Geheebia gigantea (Funck) Boulay) - RUS-AS: ARC-YAK $[47,239]$

- hedysariformis Otnyukova - RUS-AS: S-SIB [319], YAK [239]

- icmadophilus (Schimp. ex Müll.Hal.) R.H.Zander (Barbula acuta var. icmadophila (Schimp. ex Müll.Hal.) H.A.Crum) - RUS-EUR: NW [364, 407], CAUC [243], GEO [117, 243], ARM [243, 297], KAZ [296, 356], UZB [243], TAD [243, 295], KYR [296], RUS-AS: ARC-WSIB [136], ARCESIB [164, 212], W-SIB [265], S-SIB [69, 70, 212], E-SIB [126, 212], ARC-YAK [47, 239], YAK [239], ARC-FE [40, 212], N-FE [40, 140] - $\{66\}$

- insulanus (De Not.) M.O.Hill (Barbula cylindrica (Taylor) Schimp., B. vinealis var. cylindrica (Taylor) Boulay, Didymodon vinealis var. flaccidus (Bruch \& Schimp.) R.H.Zander) - LTV [244], LAT [3], EST [237], BEL [355], UKR: C [66], M [66], K [66, 328], RUS-EUR: CAUC [243], GEO [117, 243], ARM [243, 297], AZE [277], KAZ [157, 159],TUR [295, 296], TAD [294, 295], KYR [296], 
RUS-AS: S-SIB [69, 70], S-FE [76]

- johansenii (R.S.Williams) H.A.Crum (Barbula johansenii R.S.Williams) - TAD [243, 295], KYR [296], RUS-AS: S-SIB [70, 212, 323], E-SIB [74, 212], ARC-YAK [47, ?239], YAK [239], ARC-FE $[40,212,323]$

- luridus Hornsch. (Barbula lurida Hornsch., D. trifarius auct. non (Hedw.) Röhl.) - UKR: C [66], M [66], K [66, 328], GEO [117, 243], ARM [297], AZE [277, 243], TUR [295, 296], TAD [295], KYR [296]

- maximus (Syed \& Crundw.) M.O.Hill (Barbula reflexa var. robusta Braithw.) - RUS-AS: ARC-FE $[40,212]$

- murrayae Otnyukova - RUS-AS: S-SIB [212, 323]

- perobtusus Broth. - RUS-AS: S-SIB [69, 70, 212], N-FE [140]

- rigidulus Hedw. (Barbula rigidula (Hedw.) Milde) - LTV [244], LAT [3], EST [237], BEL [355], UKR: C [66], M [66], K [66, 243, 328], MLD [367], RUS-EUR: ARC [47, 150, 415], NW [207, 212, $364,407]$, NE [207, 212, 415], C [207, 212], N-UR [150, 207, 212], S-UR [150, 207, 212], SE [207, 212], CAUC [55, 212, 231, 243, 248], GEO [117, 243], ARM [243, 297], AZE [277], KAZ [156, 159, 243], TUR [11, 368], UZB [243], TAD [243, 294, 295], KYR [243, 296], RUS-AS: ARC-ESIB [164], W-SIB [265], S-SIB [69, 70, 212], E-SIB [69, 212, 275], ARC-YAK [47, 239], YAK [239], ARC-FE [40, 212], N-FE [141], S-FE [76, 212, 221]

- rigidulus var. glaucus (Ryan) Wijk \& Margad. - RUS-AS: S-FE [76]

— rigidulus var. validus (Limpr.) Düll - UKR: C [66], KYR [243]

- sinuosus (Mitt.) Delogne (Barbula sinuosa (Mitt.) Grav.) - UKR: M [66], K [66, 328], RUS-EUR: CAUC [18], GEO [102, 117], AZE [243, 277]

- spadiceus (Mitt.) Limpr. (Barbula spadicea (Mitt.) Braithw.) - LAT [3], UKR: C [66], M [66], K [66, 328], RUS-EUR: SE [366], CAUC [55, 212, 243], GEO [117, 243], TAD [295], RUS-AS: S-FE [340]

- subandreaeoides (Kindb.) R.H.Zander (D. rigidulus subsp. andreaeoides (Limpr.) Wijk \& Margad.) RUS-AS: ARC-FE [40]

- tophaceus (Brid.) Lisa (Barbula tophacea (Brid.) Mitt.) - LTV [244], LAT [3], EST [237], UKR: C [66], M [66], K [66, 243, 328], MLD [367], RUS-EUR: NE [207, 415], C [207, 212, 345], S-UR [150, 207], SE [207], CAUC [232, 243], GEO [117, 243], ARM [243, 297], AZE [277], TUR [243, 267, 295, 296], UZB [347], TAD [243, 294, 295], KYR [296, 351], RUS-AS: W-SIB [307], S-SIB [212, 324]

- tophaceus var. excurrens (Broth.) Wijk \& Margad. - TUR [296, 368]

— vinealis (Brid.) R.H.Zander (Barbula vinealis Brid.) - EST [237], BEL [355], UKR: C [66], M [66], K [66, 243, 328], RUS-EUR: NE [415], S-UR [150], SE [207, 212, 243], CAUC [212, 248], GEO [117], ARM [297], AZE [243, 277], KAZ [157, 243], TUR [243, 267, 295, 296], UZB [243, 267], TAD [243, 295], KYR [243, 267, 296], RUS-AS: ARC-YAK [47], S-FE [268] - \{69\}

Diphyscium D.Mohr [Diphysciaceae]

— foliosum (Hedw.) D.Mohr - EST [237], UKR: C [66], M [66], K [66, 328], RUS-EUR: NW [207, 364, 407], KLN [309], CAUC [55, 212, 231], GEO [117], RUS-AS: N-FE [140], S-FE [115]

Discelium Brid. [Disceliaceae]

- nudum (Dicks.) Brid. - EST [237], RUS-EUR: ARC [47, 150, 415], NW [207, 364, 262, 407], N-UR [150], RUS-AS: ARC-WSIB [136], W-SIB [265], E-SIB [275], S-FE [212, 221, 275]

Distichium Bruch et al. [Ditrichaceae]

- capillaceum (Hedw.) Bruch et al. - LTV [244], LAT [3], EST [237], BEL [355], UKR: C [66], M [66], K [66, 328], RUS-EUR: ARC [47, 127, 150], NW [207, 212, 364, 407], NE [207, 212, 415], C [207, 212, 345], N-UR [150, 207, 212], S-UR [150, 207, 212], CAUC [55, 212, 231, 248], GEO [117], ARM [297], AZE [277], KAZ [104, 295, 356], TUR [295], UZB [104], TAD [294, 295], KYR [295, 349, 351], RUS-AS: ARC-WSIB [136], ARC-ESIB [164, 212, 275], W-SIB [265], S-SIB [69, 70, 212], E-SIB [69, 212, 275], ARC-YAK [47, 212, 239], YAK [212, 239], ARC-FE [40, 212], N-FE [94, 140], S-FE [76, 77, 114, 212]

- hagenii Ryan ex H.Philib. - RUS-EUR: ARC [47], RUS-AS: ARC-WSIB [136], ARC-ESIB [212], ARC-YAK [47, 239], YAK [212, 239], ARC-FE [40]

- inclinatum (Hedw.) Bruch et al. - LAT [3], EST [237], UKR: C [66], RUS-EUR: ARC [47, 150], 
NW [212, 364, 407], NE [118, 207], N-UR [150], S-UR [207], CAUC [55, 212, 231, 248], GEO [117], ARM [297], KAZ [157, 296, 356], UZB [104], TAD [267, 294, 295], KYR [296], RUS-AS: ARC-WSIB [136], ARC-ESIB [164, 212, 275], W-SIB [265], S-SIB [69, 70, 212], E-SIB [104, 212 , 275], ARC-YAK [47, 239], YAK [212, 239], ARC-FE [40, 212], N-FE [110, 140], S-FE [37, 212]

Ditrichum Timm ex Hampe [Ditrichaceae]

- cylindricum (Hedw.) Grout (Trichodon cylindricus (Hedw.) Schimp.) - LTV [244], LAT [3], EST [237], BEL [355], UKR: C [66], M [66], RUS-EUR: ARC [47, 127, 150], NW [207, 212, 364, 407], NE [207, 212, 288, 415], KLN [309], C [207, 212], N-UR [90, 150, 207, 212], S-UR [150, 207, 212], CAUC [212, 231], KAZ [157], RUS-AS: ARC-WSIB [136], ARC-ESIB [164, 212], W-SIB [265, 275], S-SIB [69, 70, 212], E-SIB [212, 275], ARC-YAK [47, 212, 239], YAK [239], ARC-FE [40], NFE [140], S-FE [76, 77, 109, 212]

- flexicaule (Schwägr.) Hampe - LTV [244], LAT [3], EST [237], UKR: C [66], M [66], K [66, 328], MLD [367], RUS-EUR: ARC [47, 127, 150], NW [207, 212, 364, 407], NE [207, 212, 415], KLN [309], C [207, 345], N-UR [150, 207, 212], S-UR [150, 207], SE [207], CAUC [55, 212, 231, 248], GEO [117], ARM [297], AZE [277], KAZ [296, 104], TAD [295], KYR [104, 296, 351], RUS-AS: ARC-WSIB [136], ARC-ESIB [164, 212, 275], S-SIB [69, 70, 212], E-SIB [69, 212, 275], ARC-YAK [47, 212, 239], YAK [212, 239], ARC-FE [40, 212], N-FE [94, 140], S-FE [76, 212, 221]

- gracile (Mitt.) Kuntze (D. crispatissimum (Müll.Hal.) Paris, D. flexicaule var. longifolium (J.E.Zetterst.) I.Hagen, D. flexicaule var. sterile (De Not.) Limpr., D. giganteum R.S.Williams) - RUS-EUR: N-UR [91], S-UR [91], RUS-AS: ARC-WSIB [136], ARC-FE [40], N-FE [40] $-\{70\}$

- heteromallum (Hedw.) E.Britton (D. homomallum (Hedw.) Hampe) - LTV [244], BEL [355], UKR: C [66], M [66], RUS-EUR: ARC [47, 150, 415], NW [207, 212, 364, 407], NE [207, 212, 415], C [207, 212], N-UR [150, 415], S-UR [150, 207, 212], CAUC [55, 212], AZE [277], RUS-AS: W-SIB [212, 265], E-SIB [212, 247], S-SIB [69, 70], ARC-YAK [47], YAK [?239], N-FE [94, 140], S-FE [76, 77, 212, 221]

- lineare (Sw.) Lindb. - EST [237], RUS-EUR: NW [87, 364], NE [212, 417], N-UR [150], RUS-AS: YAK [?239], N-FE [140], S-FE [76, 212]

- macrorhynchum Broth. - RUS-AS: S-FE [116]

— pallidum (Hedw.) Hampe - UKR: C [66], M [66], GEO [18, 117], AZE [277], RUS-AS: S-SIB [69, 70, 212], E-SIB [69], S-FE [76]

- pusillum (Hedw.) Hampe (D. tortile (Schrad.) Brockm.) - LTV [244], LAT [3], EST [237], BEL [355], UKR: C [66], M [66], RUS-EUR: ARC [127, 150], NW [207, 364, 407], NE [207, 212, 415], KLN [309], C [207, 212], N-UR [150, 207, 212], S-UR [150, 207, 212], CAUC [55, 212, 231], GEO [117], RUS-AS: W-SIB [104, 212, 265], S-SIB [197, 212], E-SIB [69, 212, 275], YAK [239], N-FE [140], S-FE [76, 77, 212]

- cf. rhynchostegium Kindb. - RUS-AS: S-FE [78, 221]

- subulatum Hampe - UKR: C [66], GEO [117]

— zonatum (Brid.) Kindb. (D. heteromallum var. zonatum (Brid.) Podp.) - RUS-EUR: NW [364]

Dolichomitriopsis S.Okamura [Lembophyllaceae]

— diversiforme (Mitt.) Nog. - RUS-AS: S-FE [77, 212, 268]

Dolichotheca see Herzogiella

Dozya Sande Lac. [Leucodontaceae]

- japonica Sande Lac. - RUS-AS: S-FE [76, 77, 203, 212]

Drepanium C.E.O.Jensen [*Amblystegiaceae] $-\{71\}$

- recurvatum (Lindb. \& Arnell) G.Roth (Hypnum recurvatum (Lindb. \& Arnell) Kindb.) - UKR: C [66], RUS-EUR: ARC [47, 150], NW [273, 364, 407], NE [207, 212, 415], N-UR [150, 207, 212], SUR [150, 207, 212], CAUC [55, 212, 231], GEO [117], AZE [277], KAZ [296], RUS-AS: ARCWSIB [136], S-SIB [69, 70, 212], E-SIB [212, 275], ARC-YAK [47, 239], YAK [212, 239], ARC-FE $[38,212]$, S-FE [212, 221]

Drepanocladus (Müll.Hal.) G.Roth [Amblystegiaceae], see also Hamatocaulis vernicosus, etc., Loeskypnum badium, Pseudocalliergon lycopodioides, P. brevifolius, etc., Sanionia uncinata, etc., Scorpidium revolutum etc., Warnstorfia fluitans, etc.

— aduncus (Hedw.) Warnst. (D. simplicissimus Warnst., D. kneffii (Bruch et al.)Warnst., D. polycarpus 
(Blandow ex Voit.) Warnst. - LTV [244], LAT [3], EST [237], BEL [355], UKR: C [66], M [66], K [66, 328], MLD [367], RUS-EUR: ARC [47, 150], NW [207, 212, 364, 407], NE [207, 212, 415], KLN [309], C [207, 212], N-UR [150, 207, 212], S-UR [150, 207], SE [207, 212], CAUC [55, 212, 231, 248], GEO [117], ARM [297], AZE [277], KAZ [23, 159, 296, 356], TUR [296], TAD [267, 294, 296], KYR [296], RUS-AS: ARC-WSIB [136], ARC-ESIB [164, 212], W-SIB [212, 265], S-SIB $[69,70,212]$, E-SIB [69, 212, 275], ARC-YAK [47, 212, 239], YAK [212, 239], ARC-FE [40, 212], N-FE [94, 140], S-FE [76, 77, 109, 212]

— arcticus (R.S.Williams) Hedenäs (Campylium arcticum (R.S.Williams) Broth., C. zemliae C.E.O.Jensen) - RUS-EUR: ARC [47, 150], N-UR [150], RUS-AS: ARC-WSIB [136], ARC-ESIB [164, 212], ESIB [163], ARC-YAK [47, 212, 239], YAK [239], ARC-FE [40, 212], N-FE [40, 140]

- latinervis Warnst. - RUS-AS: ARC-ESIB [187b], ARC-FE [40]

- longifolius (Mitt.) Broth. ex Paris (D. capillifolius (Warnst.) Warnst.) - LTV [244], LAT [187a], EST [237], RUS-EUR: ARC [187a], NW [407], NE [415], KLN [309], S-UR [150, 207], RUS-AS: W-SIB [187a], S-SIB [187a], YAK [187a], N-FE [187a]

- polygamus (Bruch et al.) Hedenäs (Campyliadelphus polygamus (Bruch et al.) Kanda, Campylium polygamum (Bruch et al.) Lange \& C.E.O.Jensen) - LTV [244], LAT [3], EST [237], BEL [355], UKR: C [66], M [66], MLD [367], RUS-EUR: ARC [47, 150], NW [207, 212, 364, 407], NE [207, 212, 415], KLN [309], C [207, 212], N-UR [150, 207], S-UR [150, 207, 212], SE [207], CAUC [55, 212, 248], GEO [117], ARM [297], KAZ [296, 356], TAD [26, 296], RUS-AS: ARC-WSIB [136], ARC-ESIB [164, 212], W-SIB [212, 265], S-SIB [69, 70, 212], E-SIB [69, 212, 275], ARC-YAK [47, 212, 239], YAK [212, 239], ARC-FE [40, 212], N-FE [94, 140], S-FE [76, 77, 109, 212]

- sendtneri (Schimp. ex H.Müll.) Warnst. - LTV [244], LAT [3], EST [237], UKR: C [66], M [66], K [66, 328], RUS-EUR: ARC [47, 150], NW [207, 212, 364, 407], NE [207, 212, 415], KLN [309], C [207, 345], N-UR [150, 207], S-UR [150, 207], SE [207, 212], CAUC [55, 212], GEO [117], ARM [297], KAZ [23, 158], TAD [26, 296], KYR [294, 296], RUS-AS: ARC-WSIB [136], ARC-ESIB [96, 212], W-SIB [265], S-SIB [69, 70, 212], E-SIB [69, 212], ARC-YAK [47, 212, 239], YAK [212, 239], ARC-FE [40, 212], N-FE [94, 140], S-FE [76, 109, 212]

- sordidus (Müll.Hal.) Hedenäs (Drepanocladus tenuinervis T.J.Kop.) - LAT [3], EST [237], RUSEUR: NW [291, 283], NE [415], RUS-AS: ARC-ESIB [164], YAK [239]

Drummondia Hook. [Drummondiaceae]

— sinensis Müll.Hal. var. ussuriensis (Broth.) Vitt - RUS-AS: S-FE [76, 212, 221]

- turkestanica Broth. - KAZ [106, 296]

Dryptodon see Grimmia ramondii (=Dryptodon patens)

Echinophyllum O'Brian [Thuidiaceae]

- sachalinense (Lindb.) O'Brian (Helodium sachalinense (Lindb.) Broth.) - RUS-AS: N-FE [94, 140], S-FE [76, 77, 109, 212]

Encalypta Hedw. [Encalyptaceae]

- affinis R.Hedw. - RUS-EUR: ARC [47, 150], NW [193, 364, 407], NE [415], N-UR [150], GEO [117, 193], AZE [193, 277], TAD [295], RUS-AS: ARC-WSIB [136], ARC-ESIB [212, 275], W-SIB [193], E-SIB [193], ARC-YAK [47, 239], YAK [239], ARC-FE [40, 193, 212], N-FE [40, 161]

- alpina Sm. - RUS-EUR: ARC [47], NW [364], CAUC [55, 212, 231, 248], GEO [117], ARM [297], AZE [277], KAZ [156, 159, 296], TAD [294, 295], KYR [193, 296, 351], RUS-AS: ARC-WSIB [136], ARC-ESIB [164, 212, 275], S-SIB [69, 70, 193, 212], E-SIB [212, 386], ARC-YAK [47, 212, 239], YAK [212, 239], ARC-FE [40, 212], N-FE [140]

- brevicolla (Bruch et al.) Ångstr. - RUS-EUR: ARC [47, 150], NW [193, 212, 364, 407], C [193], NUR [90, 150, 415], GEO [117], KAZ [296, 356], RUS-AS: ARC-WSIB [136], W-SIB [193], S-SIB [193, 212], E-SIB [167, 193, 212], ARC-YAK [47, 193, 239], YAK [239], ARC-FE [40, 193], N-FE [40], S-FE [212, 221]

- brevipes Schljakov - RUS-EUR: NW [193, 364], RUS-AS: ARC-ESIB [164], S-SIB [193], E-SIB [126, 212], ARC-YAK [47, 193, 239], ARC-FE [40, 193, 212], N-FE [40]

- ciliata Hedw. - LAT [3], EST [237], BEL [355], UKR: C [66], M [66], RUS-EUR: ARC [47, 150], NW [212, 364, 407], C [207], N-UR [90, 150, 207, 212], S-UR [150, 207, 212], SE [207, 212], CAUC 
[55, 212, 231, 248], GEO [117], ARM [297], AZE [277], KAZ [159, 296, 356], KYR [296, 349, 351], RUS-AS: W-SIB [307], S-SIB [69, 70, 212], E-SIB [69, 212, 275], ARC-YAK [47, 239], YAK [239], ARC-FE [40], N-FE [94, 140], S-FE [76, 77, 109, 212]

- intermedia Jur. - TUR [296], KYR [296]

- longicolla Bruch - RUS-AS: ARC-ESIB [164], E-SIB [163], ARC-YAK [47, 193], ARC-FE [40, 193]

- microstoma Bals.-Criv. \& De Not. - RUS-EUR: CAUC [193, 231]

- mutica I.Hagen (E. vulgaris var. mutica Brid.) - EST [237], RUS-EUR: NW [364, 407], ARC [166], RUS-AS: ARC-WSIB [136], E-SIB [162], ARC-FE [40]

- procera Bruch - RUS-EUR: ARC [47, 150], NW [193, 364, 407], NE [415], N-UR [150, 207, 212], SUR [207, 212], AZE [277], RUS-AS: ARC-WSIB [136], ARC-ESIB [164, 212, 275], W-SIB [307], S-SIB [69, 70, 212], E-SIB [193, 212, 275], ARC-YAK [47, 212, 239], YAK [212, 239], ARC-FE [40, 193, 212], N-FE [40, 94]

— rhaptocarpa Schwägr. - EST [237], UKR: C [66], K [66, 328], RUS-EUR: ARC [47, 127, 150, 415], NW [212, 364, 407], NE [207, 212, 415], C [207, 212, 345], N-UR [150, 207, 212], S-UR [150, 207, 212], CAUC [55, 212, 231, 248], GEO [117], ARM [297], AZE [277], KAZ [156, 296, 356], TUR [296], UZB [267, 296], TAD [294, 295], KYR [296, 351], RUS-AS: ARC-WSIB [136], ARC-ESIB [164, 212, 275], W-SIB [307], S-SIB [69, 70, 212], E-SIB [69, 212, 275], ARC-YAK [47, 212, 239], YAK $[212,239]$, ARC-FE [40, 212], N-FE [94, 140] - $\{72\}$

— sibirica (Weinm.) Warnst. - KAZ [296, 59, 156, 157], RUS-AS: S-SIB [69, 193, 275]

- spathulata Müll.Hal. (E. rhaptocarpa var. spathulata (Müll.Hal.) Husn.) - UKR: M [66], K [66, 328], RUS-EUR: NW [407], NE [415], C [345], CAUC [55, 193, 212, 231], ARM [297], AZE [277], KAZ [156, 157],TUR [296], TAD [295], KYR [296] - \{72\}

- streptocarpa Hedw. - LTV [244], LAT [3], EST [237], BEL [355], UKR: C [66], M [66], K [66, 328], MLD [367], RUS-EUR: ARC [415], NW [207, 212, 364, 407], NE [207, 212, 415], KLN [309], C [207, 212], N-UR [150, 207, 415], S-UR [150, 207, 212], SE [207], CAUC [55, 212, 231, 248], GEO [117], ARM [297], AZE [277], ?TAD [295], ?UZB [267], ?KYR [296] - \{73\}

- trachymitria Ripart (E. rhaptocarpa var. leptodon (Bruch) Lindb.) - RUS-EUR: ARC [47], NW [407], CAUC [231], GEO [117], ARM [297], KAZ [156], TUR [267], KYR [349, 351], RUS-AS: ARCESIB [47], E-SIB [212, 386], ARC-YAK [239], ARC-FE [40, 212] - $\{72\}$

- vulgaris Hedw. - LTV [244], LAT [3], EST [237], BEL [355], UKR: C [66], M [66], K [66, 328], MLD [367], RUS-EUR: ARC [47, 150, 415], NW [207, 407], NE [207, 212], KLN [309], C [207, 212], N-UR [150, 207], S-UR [150, 207, 212], SE [207, 212], CAUC [55, 212, 231, 248], GEO [117], ARM [297], AZE [277], KAZ [23, 157, 296], TUR [267, 296], UZB [267, 296], TAD [267, 294, 295], KYR [296, 349, 351], RUS-AS: W-SIB [265], S-SIB [70, 212], ARC-FE [40], N-FE [140] - \{74\}

Entodon Müll.Hal. [Entodontaceae]

- challengeri (Paris) Cardot (E. compressus Müll.Hal. ex Cardot, non (Hedw.) Müll.Hal.) - RUS-EUR: CAUC [248], ARM [297], RUS-AS: S-SIB [69, 70, 212], E-SIB [275], S-FE [76, 212, 221]

- cladorrhizans (Hedw.) Müll.Hal. - excluded - $\{75\}$

- concinnus (De Not.) Paris - UKR: C [66], M [66], K [66, 328], RUS-EUR: ARC [44], N-UR [90, 207, 212], S-UR [91, 207, 212], CAUC [55, 212, 231, 248], GEO [117], ARM [297], AZE [277], KAZ [156, 159, 296], KYR [349, 351], RUS-AS: ARC-ESIB [92], S-SIB [69, 70, 212], E-SIB [69, 70, 212, 275], ARC-YAK [47, 212, 239], YAK [212, 239], ARC-FE [40, 212], N-FE [140], S-FE [76, $114,212,221]$

- diversinervis Cardot - RUS-AS: S-FE [76]

- flavescens (Hook.) A.Jaeger (E. rubicundus (Mitt.) A.Jaeger) - RUS-AS: N-FE [140], S-FE [76, 212]

- giraldii Müll.Hal. (E. sinense (Dixon) Laz.) - RUS-AS: S-FE [76] - $\{76\}$

- luridus (Griff.) A.Jaeger - RUS-AS: S-FE [76, 212]

— rufescens Laz. - RUS-AS: S-FE [268]

- scabridens Lindb. - RUS-AS: S-FE [76, 109, 212]

- schleicheri (Schimp.) Demet. - RUS-EUR: C [207, 212, 345], S-UR [150, 207, 212], CAUC [212, 231, 248], ARM [297], AZE [277], RUS-AS: S-SIB [69, 70, 212], E-SIB [212, 275], YAK [239], SFE $[76,212,221]$ 
— sullivantii (Müll.Hal.) Lindb. - RUS-AS: S-FE [76]

- transbaicalensis Bard. - RUS-AS: S-SIB [69]

Entosthodon Schwägr. [Funariaceae]

— angustifolius Jur. \& Milde (E. subpallescens Laz.) - TUR [10, 267, 296], TAD [296]

- attenuatus (Dicks.) Bryhn (Funaria attenuata (Dicks.) Lindb.) - excluded - $\{77\}$

- durieui Mont. (E. pallescens Jur.) - TUR [10, 296, 368]

- fascicularis (Hedw.) Müll.Hal. (Funaria fascicularis (Hedw.) Lindb.) - LTV [244], LAT [3], BEL [?355], UKR: C [66, 413], M [66], RUS-EUR: KLN [309], CAUC [250], KAZ [157], TAD [296] — \{78\}

- handelii (Schiffn.) Laz. - TUR [10, 267, 296, 368], TAD [294]

- hungaricus (Boros) Loeske (Funaria hungarica Boros, Physcomitrium martjanovii Broth. ex I.I.Abramov) - UKR: M [66], MLD [367], RUS-EUR: SE [207, 212, 377], KAZ [296, 356], KYR [296], RUS-AS: W-SIB [339], S-SIB [229]

- muhlenbergii (Turner) Fife (Funaria calcarea Wahlenb., F. dentata Crome, F. hibernica Hook., F. mediterranea Lindb., F. muhlenbergii Turner) - UKR: M [66], K [66, 328], RUS-EUR: C [212, 304], GEO [102, 117], ARM [297], AZE [277], KAZ [157, 296, 356], TUR [267, 296], TAD [294, 296], KYR [296, 351], UZB [267], RUS-AS: S-SIB [69, 212], S-FE [76, 212]-\{79\}

- pulchellus (H.Philib.) Brugués (Funaria pulchella H.Philib.) - RUS-EUR: N-UR [212], S-UR [177], SE [207, 212, 377], KYR [349], RUS-AS: YAK [239], S-FE [37, 212] - \{79\}

Ephemerum Hampe [Ephemeraceae]

— minutissimum Lindb. - LAT [2], UKR: M [66]

- recurvifolium (Dicks.) Boulay - UKR: M [66]

- serratum (Hedw.) Hampe - LTV [244], EST [237], BEL [355], UKR: M [66, 148], MLD [367], RUS-EUR: NW [207, 262], KLN [309], C [207, 212] — \{80\}

- sessile (Bruch) Müll.Hal. - UKR: M [66]

Epipterygium Lindb. [Mielichhoferiaceae]

— rigidum Lindb. ex Broth. - GEO [102, 117]

— tozeri (Grev.) Lindb. - GEO [117], AZE [277]

Eucladium Bruch et al. [Pottiaceae]

- verticillatum (With.) Bruch et al. - LAT [3], EST [237], UKR: C [66], M [66], K [66, 328], RUSEUR: C [207], ?S-UR [150], SE [207], CAUC [55, 208, 212, 232, 248], GEO [117], AZE [277], KAZ [296], TUR [296], UZB [347], TAD [294, 295], KYR [267, 296], RUS-AS: S-FE [76, 212] — \{81\}

Eurhynchiadelphus Ignatov \& Huttunen [Brachytheciaceae]

- eustegius (Besch.) Ignatov \& Huttunen (Eurhynchium eustegium (Besch.) Dixon, Brachythecium eustegium Besch.) - RUS-AS: N-FE [140], S-FE [76, 77, 109, 212]

Eurhynchiastrum Ignatov \& Huttunen [Brachytheciaceae]

- pulchellum (Hedw.) Ignatov \& Huttunen (Eurhynchium pulchellum (Hedw.) Jenn., incl. var praecox (Hedw.) Dixon and var. diversifolium (Bruch et al.) C.E.O.Jensen) - LTV [244], LAT [3], EST [237], BEL [355], UKR: C [66], M [66], K [66, 328], MLD [367], RUS-EUR: ARC [47, 127, 150], NW [207, 212, 364, 407], NE [207, 212, 415], KLN [309], C [207, 212], N-UR [150, 207], S-UR [150, 207, 212], SE [207], CAUC [55, 208, 212, 231, 248], GEO [117], ARM [297], ARE [277], KAZ [156, 159, 296, 356], TAD [294, 296], KYR [296, 351], RUS-AS: ARC-WSIB [136], ARC-ESIB [164, 212], W-SIB [212, 265], S-SIB [69, 70, 198, 212], E-SIB [69, 212, 275], ARC-YAK [47, 212, 239], YAK [212, 239], ARC-FE [40, 212], N-FE [94, 140], S-FE [76, 109, 212, 221]

Eurhynchium Bruch et al. [Brachytheciaceae], see also Eurhynchiadelphus eustegius, Eurhynchiastrum pulchellum, Kindbergia praelonga, Oxyrrhynchium hians, etc., Plasteurhynchium striatulum, Scorpiurium circinatum

- angustirete (Broth.) T.J.Kop. (E. striatum subsp. zetterstedtii (P.Størmer) Podp., E. striatum var. pachycladum G.Roth) - LTV [244], LAT [3], EST [237], BEL [355], UKR: C [66], M [66], K [66, 328], RUS-EUR: NW [207, 407], KLN [309], C [207, 212], S-UR [207], CAUC [55, 208, 212, 231, 248], GEO [117], ARM [297], AZE [277], KAZ* (sub striatum) [296], KYR* (sub striatum) [296], RUSAS: S-SIB [70, 198, 212]

— striatum (Hedw.) Schimp. - LTV [244], LAT [3], EST [397], BEL [355], UKR: C [66], M [66], K 
[66, 328], RUS-EUR: KLN [?309], CAUC [208, 248], GEO [117], ARM [297], AZE [277], KAZ [159] $-\{82\}$

Eurohypnum Ando [Hypnaceae]

— leptothallum (Müll.Hal.) Ando - RUS-AS: S-SIB [69, 70, 212], E-SIB [69, 212], S-FE [76, 212, 221]

Fabronia Raddi [Fabroniaceae]

- ciliaris (Brid.) Brid. - RUS-EUR: S-UR [150, 207], CAUC [102, 248, 250], GEO [102, 117], ARM [297], AZE [277], KAZ [296, 356], KYR [296], RUS-AS: S-SIB [69, 70, 212], YAK [239], S-FE [76, $212,221]$

- matsumurae Besch. - excluded - $\{83\}$

— pusilla Raddi - RUS-EUR: SE [207], CAUC [55, 212, 231], GEO [117], ARM [297], TUR [296], TAD [294, 296], KYR [296, 349, 351] - \{84\}

Fauriella Besch. [*Pylaisiadelphaceae]

— tenuis (Mitt.) Cardot - RUS-AS: S-FE [76, 77, 109, 212]

Fissidens Hedw. [Fissidentaceae]

— adianthoides Hedw. - LTV [244], LAT [3], EST [237], BEL [355], UKR: C [66], M [66], K [66, 328], RUS-EUR: ARC [150], NW [207, 212, 364, 407], NE [207, 212, 415], KLN [309], C [207, 212], N-UR [150, 207, 212], S-UR [150, 207, 212], SE [207], CAUC [55, 212, 231, 248], GEO [117], ARM [297], AZE [277], RUS-AS: ARC-ESIB [93], W-SIB [265], S-SIB [69, 70, 212], E-SIB [69, 212], ARC-YAK [47, 239], YAK [239], ARC-FE [40, 212], N-FE [140, 109]

— arcticus Bryhn - RUS-AS: ARC-WSIB [136, 134], ARC-ESIB [134], ARC-YAK [49] — \{85)

— arnoldii R.Ruthe - LTV [244], LAT [3], EST [237], UKR: C [66], M [66], TUR [296]

- bryoides Hedw. - LTV [244], LAT [3], EST [237], BEL [355], UKR: C [66], M [66], K [66, 328], MLD [367], RUS-EUR: ARC [47, 127, 150], NW [207, 364, 407], NE [207, 212, 415], KLN [309], C [207, 212], N-UR [150, 207, 212], S-UR [150, 207, 212], SE [207], CAUC [55, 208, 212, 231, 248], GEO [117], ARM [297], AZE [277], KAZ [156, 296, 356], TUR [296], TAD [294, 295], KYR [296], RUSAS: ARC-WSIB [136], ARC-ESIB [93, 163], W-SIB [212, 265], S-SIB [69, 70, 212], E-SIB [69, 212], ARC-YAK [47, 239], YAK [212, 239], ARC-FE [40], N-FE [40, 140], S-FE [76, 212, 221]

- bryoides var. gymnandrus (Büse) R.Ruthe (F. gymnandrus Büse) - LAT [1], RUS-EUR: NW [84, 407], C [207], S-UR [91], TAD [295] - \{86\}

- crassipes Wilson ex Bruch et al. (F. mildeanus Schimp.) - LAT [3], UKR: M [66], K [328], RUSEUR: S-UR [150], GEO [117], AZE [277], TUR [296]

- crispus Mont. (F. limbatus Sull., F. bambergeri auct. non Milde) - UKR: K [328], TUR [296]

- curvatus Hornsch. (F. strictulus Müll.Hal.) - RUS-AS: S-FE [221]

- dubius P.Beauv. (F. cristatus Wilson ex Mitt., F. decipiens De Not.) - LTV [244], LAT [3], EST [237], UKR: C [66], M [66], K [66, 328], MLD [367], RUS-EUR: NW [207], KLN [309], C [207], CAUC [55, 208, 212, 231, 248], GEO [117], ARM [297], AZE [277], RUS-AS: N-FE [140], S-FE [76, 77, $109,212]$

— exiguus Sull. - UKR: M [66], RUS-EUR: C [207, 212, 345], S-UR [88, 207], TUR [296], TAD [295]

- exilis Hedw. - LTV [244], EST [237], BEL [355], UKR: C [66], M [66]), MLD [367], RUS-EUR: NW [207], NE [207], KLN [309], C [207, 212, 345], S-UR [89, 207, 275], CAUC [248], GEO [117], TAD [294, 295], RUS-AS: S-SIB [427]

- fontanus (Bach.Pyl.) Steud. (Octodiceras fontanum (Bach.Pyl.) Lindb.) - LAT [3], EST [237], UKR: M [66, 402], RUS-EUR: NW [262]

— gracilifolius Brugg.-Nann. \& Nyholm (F. viridulus var. tenuifolius (Boulay) A.J.E.Sm., F. minutulus auct. non Wils.) - EST [237], MLD [367], UKR: C [66], M [66]), RUS-EUR: NW [63], NE [415], C [207, 212, 345], S-UR [150, 207], SE [207], CAUC [55, 208, 212, 232, 248], GEO [13], RUS-AS: ?ARC-YAK [47], ?S-FE [76] - $\{87\}$

- grandifrons Brid. (Pachyfissidens grandifrons (Brid.) Limpr.) - ARM [297], KAZ [157, 296], KYR [267], UZB [296, 347], TAD [294, 295]

- gymnogynus Besch. - RUS-AS: S-FE [174, 178]

— incurvus Starke ex Röhl. (F. viridulus var. incurvus (Starke ex Röhl.) Waldh.) - UKR: M [66], GEO [117] 
— karataviensis N.Samsel - KAZ [295], UZB [296], KYR [296] $-\{88\}$

- marginatulus Meln. - UKR: C [66], M [66], K [328], RUS-EUR: NE [415]

- nobilis Griff. (F. japonicus Dozy \& Molk.) - RUS-AS: S-FE [77]

— osmundoides Hedw. - LTV [244], LAT [3], EST [237], BEL [355], UKR [392], RUS-EUR: ARC [47, 150], NW [207, 212, 364, 407], NE [207, 212, 415], KLN [309], C [207, 212], N-UR [90, 150, 207, 212], S-UR [150, 207, 212], CAUC [55, 212, 231], AZE [277], KAZ [296], KYR [296], RUSAS: ARC-WSIB [136], ARC-ESIB [164, 212], W-SIB [265], S-SIB [69, 70, 212], E-SIB [212, 275], ARC-YAK [47, 239], YAK [239], ARC-FE [40, 212], N-FE [40, 140], S-FE [221] - \{89\}

- persicus R.Ruthe - TUR [296, 368]

- pusillus (Wilson) Milde - LAT [3], EST [237], UKR: C [66], M [66], RUS-EUR: NW [285], NE [415], KLN [309], ?C [212, 345], S-UR [150], CAUC [18], GEO [117], ARM [297] - \{90\}

- rivularis (Spruce) Bruch et al. - UKR: K [328], RUS-EUR: CAUC [55, 212], GEO [117]

- rufulus Bruch et al. - UKR: C [66], RUS-EUR: NE [415]

— taxifolius Hedw. - LTV [244], LAT [3], EST [237], BEL [355], UKR: C [66], M [66], K [66, 328], MLD [367], RUS-EUR: NW [207], NE [207], KLN [309], C [207, 212], S-UR [150, 207, 212], SE [207], CAUC [55, 208, 212, 231, 248], GEO [117], ARM [297], AZE [277], RUS-AS: S-SIB [70, 212], S-FE [76, 77, 212]

- teysmannianus Dozy \& Molk. (F. adelphinus Besch.) - RUS-AS: S-FE [77]

— viridulus (Sw.) Wahlenb. - LTV [244], UKR: C [66], M [66], MLD [367], UKR: C [66], M [66]), RUS-EUR: NW [212, 364, 407], NE [212, 415], S-UR [91], GEO [117], AZE [277], RUS-AS: ARCWSIB [136], ARC-ESIB [93, 164, 212], W-SIB [265], S-SIB [212, 336], E-SIB [163], ARC-YAK [47, 239], YAK [239], ARC-FE [40, 212], N-FE [40, 140]

Fontinalis Hedw. [Fontinalaceae]

— antipyretica Hedw. - LTV [244], LAT [3], EST [237], BEL [355], UKR: C [66], M [66], K [66, 328], RUS-EUR: ARC [47, 150], NW [207, 212, 364, 407], NE [207, 212, 415], KLN [309], C [207, 212], N-UR [150, 207, 212], S-UR [150, 207, 212], SE [207, 212], CAUC [55, 208, 212, 231, 248], GEO [117], ARM [297], AZE [277], KAZ [156, 296, 356], TAD [296], KYR [296, 351], RUS-AS: ARCESIB [164], W-SIB [212, 265], S-SIB [69, 70, 212], E-SIB [69, 212, 275], ARC-YAK [47, 239], YAK [212, 239], ARC-FE [40], N-FE [94, 140], S-FE [76, 212]

- antipyretica var. gracilis (Lindb.) Schimp. - LTV [244], LAT [1], EST [237], BEL [355], RUS-EUR: NW [85, 212, 251, 364, 407], NE [414], KLN [309], C [207], N-UR [150], S-UR [150, 212], RUS-AS: ARC-ESIB [164], S-SIB [197], E-SIB [275], YAK [239], N-FE [140], S-FE [79, 212]

- dalecarlica Bruch et al. - LTV [244], LAT [3], EST [237], RUS-EUR: ARC [150], NW [207, 212, 364, 407], NE [207, 212, 415], C [207, 212], N-UR [90, 150, 207, 212, 415], S-UR [150, 207, 212] - \{91\}

- hypnoides Hartm. - LTV [244], LAT [3], EST [237], BEL [355], UKR: C [66], M [66], RUS-EUR: ARC [47, 150], NW [207, 407], NE [207, 212, 415], C [207, 212], N-UR [150, 207, 415], S-UR [150, 207], ARM [297], AZE [277], KAZ [296, 356], RUS-AS: ARC-ESIB [164, 212], W-SIB [212, 265], S-SIB [69, 70, 212], E-SIB [212, 275], ARC-YAK [47, 239], YAK [212, 239], N-FE [140], S-FE [114]

- hypnoides var. duriaei (Schimp.) Kindb. - RUS-EUR: NE [414], N-UR [150], KAZ [23], KYR [267, 296], RUS-AS: S-SIB [69]

- squamosa Hedw. - LTV [244], EST [237], RUS-EUR: NW [212, 364, 407], NE [414], N-UR [150, 415], S-UR [150], RUS-AS: S-SIB [197], E-SIB [212, 247]

Forsstroemia Lindb. [Neckeraceae]

- cryphaeoides Cardot - RUS-AS: S-FE [76, 203, 212]

- japonica (Besch.) Paris - RUS-AS: S-FE [76, 77, 109, 203, 212]

- noguchii L.R.Stark - RUS-AS: S-SIB [203, 374]

- stricta Laz. - RUS-AS: S-FE [203, 212, 268]

- trichomitria (Hedw.) Lindb. - RUS-AS: S-FE [76, 203, 212]

Funaria Hedw. [Funariaceae], see also Entosthodon muhlenbergii, E. pulchellus

- aequidens Lindb. ex Broth. - RUS-EUR: CAUC [25, 102], GEO [25, 117], KAZ [25, 157], TAD [25, 296]

- arctica (Berggr.) Kindb. - RUS-AS: ARC-WSIB [136], ARC-ESIB [164], ARC-YAK [47, 212, 239], ?ARC-FE [35] 
- hygrometrica Hedw. - LTV [244], LAT [3], EST [237], BEL [355], UKR: C [66], M [66], K [66, 328], MLD [367], RUS-EUR: ARC [47, 127, 150], NW [207, 212, 364, 407], NE [207, 212, 415], KLN [309], C [207, 212], N-UR [90, 150, 207], S-UR [150, 207, 212], SE [207], CAUC [55, 208, 212 , 231, 248], GEO [117], ARM [297], AZE [277], KAZ [156, 296, 356], TUR [267, 296], TAD [294, 296], KYR [267, 296, 351], RUS-AS: ARC-WSIB [136], ARC-ESIB [164, 212], W-SIB [212, 265], S-SIB [69, 70, 212], E-SIB [69, 212, 275], ARC-YAK [47, 239], YAK [212, 239], ARC-FE [40, 212], N-FE [94, 140], S-FE [76, 77, 109, 212]

— microstoma Bruch ex Schimp. - UKR: M [66], RUS-EUR: ?NE [415], TAD [294, 296], KYR [296] $-\{92\}$

— polaris Bryhn - RUS-AS: ARC-FE [40, 212]

Glyphomitrium Brid. [Ptychomitriaceae]

- humillimum (Mitt.) Cardot - RUS-AS: S-FE [76, 77, 114, 212]

Gollania Broth. [Pylaisiaceae]

- ruginosa (Mitt.) Broth. - RUS-AS: S-FE [76, 212, 221]

— turgens (Müll.Hal.) Ando - RUS-AS: S-SIB [69, 70, 212]

Grimmia Hedw. [Grimmiaceae], see also Schistidium apocarpum, S. alpicola, etc.

- alpestris (F.Weber \& D.Mohr) Schleich. - UKR: C [66, 306], RUS-EUR: S-UR [150, 234], CAUC [55, 231, 234, 248], GEO [117, 306], ARM [297, 306], AZE [277], KAZ [159, 296, 306, 356], UZB [306], TAD [294, 296, 306], KYR [296], RUS-AS: S-SIB [234], N-FE [140, 234], S-FE [221]

— anodon Bruch et al. - UKR: M [66], K [66, 328], RUS-EUR: NW [234, 407], C [234], S-UR [234], SE [234], CAUC [55, 231, 234, 248], GEO [117, 306], ARM [297, 306], AZE [277], KAZ [159, 296, 306], TUR [267, 296], TAD [294, 296], KYR [267, 296, 306], UZB [267], RUS-AS: ARC-ESIB [164, 234], S-SIB [70, 234], E-SIB [234], ARC-YAK [234], YAK [234, 239], ARC-FE [40, 234]

- anomala Hampe ex Schimp. - UKR: C [66], RUS-EUR: NW [234, 87], S-UR [234], CAUC [55, 231, 234], GEO [117, 306], RUS-AS: S-SIB [234], N-FE [140]

- atrata Miel. ex Hornsch. (Streptocolea atrata (Miel. ex Hornsch.) Ochyra \& Żarnowiec) - excluded [66]

- caespiticia (Brid.) Jur. - RUS-EUR: CAUC [55, 231, 234], GEO [117, 306], ARM [297, 306], KYR [296], UZB [267], RUS-AS: S-SIB [234]

- capillata De Not. (G. mesopotamica Schiffn.) - TUR [306], TAD [295, 296], RUS-AS: S-SIB [234] $-\{93\}$

— crinita Brid. - EST [237], GEO [117, 306], ARM [297], AZE [277, 306], TUR [296, 306], KYR [296]

— decipiens (Schultz) Lindb. - UKR: C [66], K [66, 328], ARM [297, 306]

- donniana Sm. - UKR: C [66, 306], RUS-EUR: NW [234, 273, 364, 407], N-UR [234, 415], CAUC [234, 248], GEO [117, 306], ARM [297], AZE [277], TAD [296], KYR [296, 351], RUS-AS: S-SIB [234], E-SIB [234], YAK [234, 239], ARC-FE [40, 234], N-FE [140, 234], S-FE [221]

- elatior Bruch ex Bals.-Criv. \& De Not. - UKR: C [66], RUS-EUR: NW [234, 364, 407], NE [234], C [207], N-UR [90, 150, 234], S-UR [150, 234], CAUC [55, 231, 234], GEO [117, 248, 306], ARM [297, 306], AZE [277], KAZ [23, 159, 296, 306, 356], TAD [296], KYR [296, 351, 306], RUS-AS: ARC-ESIB [164, 234], S-SIB [69, 234], E-SIB [234], ARC-FE [40, 234], S-FE [221, 234]

- elongata Kaulf. - UKR: C [66], K [328], RUS-EUR: N-UR [234], GEO [117, 306], ARM [297], KAZ [159, 296, 356], TAD [296], KYR [296], RUS-AS: N-FE [141]

- exquisita J.Muñoz - TUR [305], TAD [305]

- funalis (Schwägr.) Bruch et al. - UKR: C [66, 306], RUS-EUR: NW [234, 364], N-UR [234], CAUC [55, 231, 234, 248], GEO [117, 306], ARM [297, 306], AZE [277, 306], KAZ [296, 356], TUR [296], TAD [296], RUS-AS: ARC-ESIB [164, 234], S-SIB [234], E-SIB [234], YAK [234, 239], ARCFE [40, 234], S-FE [221, 234]

- fuscolutea Hook. (G. apiculata Hornsch.) - UKR: C [66], RUS-EUR: CAUC [231, 234], RUS-AS: S-SIB [234, 306], N-FE [141]

- hartmanii Schimp. (G. brotheri Lindb. ex Broth.) - LAT [3], EST [237], UKR: C [66], M [66], K [328], RUS-EUR: NW [234, 407], S-UR [234], KLN [309], CAUC [55, 231, 234, 248], GEO [117, 306], ARM [297, 306], RUS-AS: N-FE [140, 234], S-FE [77, 109, 234]

— incurva Schwägr. - UKR: C [66, 306], RUS-EUR: NW [234, 273, 364, 407], NE [234], N-UR [150, 
234], S-UR [150, 234], CAUC [55, 231, 234, 248], GEO [117, 306], ARM [297], RUS-AS: ARCESIB [164, 234], S-SIB [70, 234], YAK [234, 239], ARC-FE [40, 234], N-FE [94, 140, 234], S-FE [221, 234]

- jacutica Ignatova, Bednarek-Ochyra, Afonina \& J.Muñoz - RUS-AS: ARC-ESIB [164, 225, 234], SSIB [225, 234], E-SIB [225, 234], ARK-YAK [239], YAK [225, 234, 239], ARC-FE [225, 234], S-FE $[225,234]$

- laevigata (Brid.) Brid. - BEL [355], UKR: C [66], M [66], K [66, 328], RUS-EUR: NW [234], C [234, 345], S-UR [150, 234], SE [234], CAUC [231, 232, 234, 248], GEO [117, 306], ARM [297, 306], AZE [277], KAZ [267, 296, 306, 356], TUR [296], UZB [267, 306], TAD [294, 296, 306], KYR [296, 306, 351], RUS-AS: S-SIB [69, 70, 234]

- longirostris Hook. (G. affinis Hornsch., G. cavifolia Lindb. \& Arnell) - UKR: C [66], M [66], RUSEUR: ARC [127, 150], NW [87, 234, 364, 407], NE [234], N-UR [90, 150, 234], S-UR [150, 234], CAUC [55, 231, 234, 248], GEO [117, 306], KAZ [306], TUR [306], KYR [351], RUS-AS: ARCWSIB [136], ARC-ESIB [164], W-SIB [234], S-SIB [69, 70, 234], E-SIB [69, 234], ARC-YAK [239], YAK [234, 239], ARC-FE [40, 234], N-FE [94, 140, 234], S-FE [221, 234]

- mollis Bruch et al. (Hydrogrimmia mollis (Bruch et al.) Loeske) - RUS-EUR: NW [87, 234, 364], NUR [150, 234], CAUC [231, 234], GEO [117], RUS-AS: S-SIB [197, 234], YAK [234, 239], ARC-FE [40, 234], N-FE [94, 140, 234], S-FE [221, 234]

- montana Bruch et al. - UKR: C [66], RUS-EUR: NW [234, 364, 407], CAUC [231, 234], GEO [117], ARM [297], AZE [277], KAZ [296, 356], TAD [296], KYR [296, 351] - \{94\}

- muehlenbeckii Schimp. - LAT [306], BEL [355], UKR: C [66], M [66], K [66, 328], RUS-EUR: ARC [150], NW [234, 364, 407], NE [234, 288], KLN [309], C [234, 345], N-UR [150, 234], S-UR [150, 234], CAUC [231, 234, 248], GEO [306], AZE [277], RUS-AS: W-SIB [234], S-SIB [69, 234], E-SIB [234, 275], YAK [234, 239], S-FE [234] - \{95\}

- orbicularis Bruch ex Wilson - UKR: M [66], K [66, 328], RUS-EUR: CAUC [234], GEO [117, 306], ARM [297, 306], AZE [306], KAZ [296], TUR [267, 296, 306], UZB [296, 306], TAD [296], KYR [296]

- ovalis (Hedw.) Lindb. - LTV [244], LAT [3], EST [237], BEL [355], UKR: C [66], M [66], K [66, 328], RUS-EUR: NW [234, 407], C [234], S-UR [150, 234], SE [234], CAUC [231, 234, 248], GEO [306], ARM [297, 306], AZE [277], KAZ [23, 296, 306, 356], TUR [296], UZB [267, 306], TAD [294, 296, 306], KYR [296, 306, 351], RUS-AS: S-SIB [70, 234], N-FE [140], S-FE [221, 234] - \{96\}

— pilifera P.Beauv. - RUS-AS: S-SIB [234], ARC-FE [40, 234], S-FE [221, 234]

- plagiopodia Hedw. - UKR: M [66, 306], RUS-EUR: C [423], S-UR [150, 234], SE [234], CAUC [250], GEO [117, 306], ARM [297, 306], AZE [277], TAD [294, 296], KYR [296, 349], RUS-AS: SSIB [70, 234]

— poecilostoma Cardot \& Sebille (G. tergestina var. poecilostoma (Cardot \& Sebille) Loeske) - UKR: K [66, 306, 328], RUS-EUR S-UR [52], (CAUC [234, 248], GEO [306], ARM [297, 306], AZE [306], KAZ [306], TUR [306], TAD [296], KYR [267, 296, 306], UZB [267], RUS-AS: S-SIB [234], YAK $[234,239]$

- pulvinata (Hedw.) Sm. (G. pulvinata var. africana (Hedw.) Hook.f. \& Wilson) - LTV [244], LAT [3], EST [237], BEL [355], UKR: C [66], M [66], K [66, 328], MLD [367], RUS-EUR: NW [234, 407], KLN [309], C [234], SE [234], CAUC [55, 208, 231, 232, 234, 248], GEO [117, 306], ARM [297, 306], AZE [277], KAZ [159, 296, 356], TUR [296, 306], UZB [267, 296, 306], TAD [294, 296, 306], KYR [296, 351]

- ramondii (Lam. \& DC.) Margad. (Racomitrium patens (Hedw.) Huebener, Dryptodon patens (Hedw.) Brid., G. curvata (Brid.) De Sloover) - LAT [3], UKR: C [66], RUS-EUR: NW [234, 407], C [234], GEO [117], ARM [297], AZE [277]

- reflexidens Müll.Hal. (G. sessitana De Not., G. subsulcata Limpr., G. laevidens Broth.) - RUS-EUR: NW [234], N-UR [150, 234, 415], CAUC [208, 231, 234, 248], GEO [117, 306], KAZ [306], KYR [296], UZB [267], RUS-AS: S-SIB [234], E-SIB [163, 234], YAK [234, 239], ARC-FE [40, 234], NFE [140, 234], S-FE [234] - \{97\}

- teretinervis Limpr. - RUS-EUR: N-UR [234], S-UR [234], CAUC [234], RUS-AS: E-SIB [234], ARC-YAK [234, 239], YAK [234, 239] 
— tergestina Tomm. ex Bruch et al. (G. tergestinoides Culm., G. crassifolia Lindb. ex Broth.) - UKR: M [66], K [328], RUS-EUR: N-UR [?234], S-UR [?234], CAUC [231, 232, 234, 248], GEO [117, 306], ARM [297, 306], KAZ [296, 356], TUR [267, 296, 306], UZB [385], TAD [294, 296], KYR [296], RUS-AS: S-SIB [234], YAK [234, 239], S-FE [234] - $\{98\}$

- torquata Drumm. - RUS-EUR: ARC [150], NW [234, 273, 364, 407], N-UR [234], CAUC [231, 234], RUS-AS: YAK [234, 239], ARC-ESIB [50], ARC-FE [40, 234], N-FE [141, 234], S-FE [234]

- trichophylla Grev. - LTV [244], LAT [3], EST [237], BEL [355], UKR: C [66], M [66], K [66, 328], RUS-EUR: KLN [309], GEO [117], ARM [297] — \{99\}

— triformis Carestia \& De Not. - RUS-AS: S-SIB [234, 306], N-FE [141]

- unicolor Hook. - RUS-EUR: NW [234, 407], CAUC [231, 234], GEO [117], AZE [277], KAZ [159, 296, 306, 356], TUR [296], TAD [296], KYR [296], RUS-AS: S-SIB [69, 70, 234], S-FE [221, 234] — \{100\}

Gymnostomum Nees \& Hornsch. [Pottiaceae], see also Hymenostylium recurvirostrum

— aeruginosum Sm. - LAT [3], EST [237], UKR: C [66], M [66], K [328], RUS-EUR: NW [212, 364, 407], NE [207, 212, 415], C [207, 345], N-UR [90, 150, 207, 415], S-UR [150, 207, 212], SE [366], CAUC [55, 208, 212, 231, 248], GEO [117], ARM [297], AZE [277], KAZ [156, 157], UZB [347], TAD [294, 295], KYR [296, 351], RUS-AS: S-SIB [69, 70, 212], E-SIB [162, 212, 386], ARC-YAK [47, 239], YAK [239], ARC-FE [40], S-FE [76, 77, 212] - \{101\}

— boreale Nyholm \& Hedenäs - RUS-EUR: NW [291, 314], RUS-AS: E-SIB [163] - $\{102\}$

- calcareum Nees \& Hornsch. - LAT [3], EST [237], UKR: M [66], K [66, 328], RUS-EUR: NW [407], CAUC [208, 212, 248], GEO [117], ARM [297], AZE [277], UZB [347], TAD [294, 295], KYR [296, 349], RUS-AS: S-SIB [197, 212], ARC-YAK [239], YAK [239] - $\{101\}$

- calcareum var. viridulum Brid. - RUS-EUR: CAUC [102], GEO [102], TUR [296, 368]

Gyroweisia Schimp. [Pottiaceae]

- tenuis (Hedw.) Schimp. - LTV [244], LAT [3], EST [237], UKR: C [66], M [66], K [328], RUSEUR: NW [207], C [207, 212, 345], S-UR [89, 207], SE [207], CAUC [250], RUS-AS: S-SIB [69, 71] Habrodon Schimp. [Habrodontaceae], see also Iwatsukiella leucotricha

- perpusillus (De Not.) Lindb. - RUS-EUR: CAUC [228, 232]

Hamatocaulis Hedenäs [Scorpidiaceae]

- lapponicus (Norrl.) Hedenäs (Drepanocladus lapponicus (Norrl.) Smirnova) - LAT [3], RUS-EUR: ARC [47, 150], NW [86, 212, 407], NE [415], C [430], RUS-AS: ARC-WSIB [136], ARC-ESIB [212], W-SIB [265], E-SIB [126, 212], ARC-YAK [47, 239], YAK [239], ARC-FE [40], N-FE [40, 94]

- vernicosus (Mitt.) Hedenäs (Drepanocladus vernicosus (Mitt.) Warnst.) - LTV [244], LAT [3], EST [237], BEL [355], UKR: C [66], M [66], RUS-EUR: ARC [47, 150], NW [207, 212, 364, 407], NE [207, 212, 415], KLN [309], C [207, 212, 345], N-UR [150, 207], S-UR [150, 207, 212], CAUC [55, 212, 231, 248], GEO [117], ARM [297], KAZ [158, 296, 3568], KYR [296], RUS-AS: ARC-WSIB [136], ARC-ESIB [164, 212, 275], W-SIB [212, 265], S-SIB [69, 70, 212], E-SIB [69, 212, 275], ARC-YAK [47, 212, 239], YAK [212, 239], ARC-FE [40], N-FE [40], S-FE [76]

Haplocladium (Müll.Hal.) Müll.Hal. [Thuidiaceae]

- angustifolium (Hampe \& Müll.Hal.) Broth. (Bryohaplocladium angustifolium (Hampe \& Müll.Hal.) R.Watan. \& Z.Iwats.) - RUS-EUR: ?C: [212, 304], RUS-AS: S-SIB [197, 212], E-SIB [126, 212], SFE $[76,212,221]-\{103\}$

- microphyllum (Hedw.) Broth. (Bryohaplocladium microphyllum (Hedw.) R.Watan. \& Z.Iwats.) - BEL [355], UKR: M [66], RUS-EUR: C [207, 212, 345], S-UR [91, 150, 207, 212], RUS-AS: W-SIB [265], S-SIB [69, 70, 212], E-SIB [212, 275], S-FE [76, 77, 109, 212]

- strictulum (Cardot) Reimers - RUS-AS: S-FE [76, 212]

— virginianum (Brid.) Broth. (Bryohaplocladium virginianum (Brid.) R.Watan. \& Z.Iwats.) - GEO [102, 117], ARM [297]

Haplohymenium Dozy \& Molk. [Anomodontaceae]

- flagelliforme L.I.Savicz - RUS-AS: S-FE [76, 212]

- longinerve (Broth.) Broth. - RUS-AS: S-SIB [69, 72], S-FE [75, 79, 212]

- triste (Ces.) Kindb. (Anomodon tristis (Ces.) Sull. \& Lesq. - GEO [28], RUS-AS: S-SIB [69, 70], YAK [239], S-FE [76, 212, 221] 
Hedwigia P.Beauv. [Hedwigiaceae]

- ciliata (Hedw.) P.Beauv. - LTV [244], LAT [3], EST [237], BEL [355], UKR: C [66], M [66], K [66, 328], RUS-EUR: ARC [47, 150, 415], NW [207, 212, 364, 407], NE [53, 207, 415], KLN [309], C [207, 212, 345], N-UR [150, 207, 212], S-UR [150, 207, 212], SE [207], CAUC [55, 212, 231, 248], GEO [117], ARM [297], AZE [277], KAZ [156, 296, 356], TAD [296], KYR [296], RUS-AS: S-SIB [69, 70, 212], E-SIB [69, 162, 212], YAK [212, 239], ARC-FE [40], N-FE [40, 161], S-FE [76, 109, 212]

- stellata Hedenäs - RUS-AS: S-FE [221]

Helodium Warnst. [Thuidiaceae], see also Echinophyllum sachalinense

- blandowii (F.Weber \& D.Mohr) Warnst. - LTV [244], LAT [3], EST [237], BEL [355], UKR: M [66], RUS-EUR: ARC [47, 127, 150], NW [207, 212, 364, 407], NE [207, 212, 415], KLN [309], C [207, 212], N-UR [150, 207, 212], S-UR [150, 207, 212], SE [207], CAUC [248], TAD [26, 296], KYR [296], RUS-AS: ARC-WSIB [136], ARC-ESIB [212], W-SIB [212, 265], S-SIB [69, 70, 212], E-SIB [69, 212, 275], ARC-YAK [239], YAK [212, 239], ARC-FE [40], N-FE [94, 140], S-FE $[109,212]$

— paludosum (Austin) Broth. - RUS-AS: S-SIB [69, 212], E-SIB [212, 247], S-FE [76, 77, 212]

Hennediella Paris [Pottiaceae]

- heimii (Hedw.) R.H.Zander (Desmatodon heimii (Hedw.) Mitt., Pottia heimii (Hedw.) Hampe) - LAT [3], EST [237], UKR: M [66], RUS-EUR: ARC [47], NW [364], C [207, 345], AZE [277], KAZ [157, 296], TAD [295], KYR [296], RUS-AS: ARC-WSIB [136], ARC-ESIB [212, 275], S-SIB [70, 197], ARC-YAK [47, 212, 239], ARC-FE [40, 212]

- heimii var. arctica (Lindb.) R.H.Zander - RUS-EUR: ARC [47, 150], TAD [295], RUS-AS: ARCESIB [164], E-SIB [163], ARC-YAK [47, 239], ARC-FE [40, 212], N-FE [140]

Herpetineuron (Müll.Hal.) Cardot [Anomodontaceae]

— toccoae (Sull. \& Lesq.) Cardot - RUS-AS: S-SIB [69], S-FE [76, 212]

Herzogiella Broth. [Plagiotheciaceae]

— adscendens (Lindb.) Z.Iwats. \& W.B.Schofield - RUS-AS: ARC-FE [40], N-FE [94, 140], S-FE [76, $77,109,212,274]$

- seligeri (Brid.) Z.Iwats. - LTV [244], LAT [3], EST [237], BEL [355], UKR: C [66], M [66], K [66, 328], MLD [367], RUS-EUR: NW [207, 407], NE [207, 212], KLN [309], C [207, 212], S-UR [150, 207, 212], SE [207], CAUC [55, 208, 212, 231, 248], GEO [117], ARM [297], AZE [277], KAZ (156, 159), TAD [296], KYR [296]

- striatella (Brid.) Z.Iwats. - LAT [4], EST [237], UKR: C [66], RUS-EUR: ARC [47, 150], NW [364, 407], N-UR [?47], RUS-AS: S-SIB [336], S-FE [76, 212]

- turfacea (Lindb.) Z.Iwats. - RUS-EUR: NW [207, 212, 364, 407], NE [207, 212, 415], C [207, 212], N-UR [150, 207, 415], S-UR [150, 212], RUS-AS: W-SIB [212, 265], S-SIB [69, 70, 212], E-SIB $[212,275]$, ?ARC-YAK [47], YAK [239], S-FE [76, 77, 109, 212] - $\{104\}$

Heterocladium Bruch et al. [Heterocladiaceae], see also Pseudoleskeella papillosa

- dimorphum (Brid.) Bruch et al. - UKR: C [66], RUS-EUR: ARC [47, 150], NW [212, 273, 364, 407], NE [207, 288, 416], N-UR [150, 207], S-UR [150], CAUC [55, 212, 231], GEO [117], AZE [277], RUS-AS: W-SIB [265], S-SIB [69, 212], N-FE [140], S-FE [110]

- heteropterum (Brid.) Bruch et al. - UKR: C [66], RUS-EUR: CAUC [55, 212, 231], GEO [117], AZE [277]

— procurrens (Mitt.) A.Jaeger - RUS-AS: ARC-FE [40]

Heterophyllium (Schimp.) Kindb. [Pylaisiadelphaceae], see also Callicladium haldanianum

- affine (Hook.) M.Fleisch. - UKR: C [66], RUS-EUR: CAUC [208, 212], GEO [102], RUS-AS: SSIB [69, 70, 212], E-SIB [212, 247], S-FE [76, 212, 221]

Hilpertia R.H.Zander [Pottiaceae]

— velenovskyi (Schiffn.) R.H.Zander - RUS-EUR: CAUC [384, 250], RUS-AS: E-SIB [7]

Homalia Brid. [Neckeraceae], see also Neckera besseri

- trichomanoides (Hedw.) Bruch et al. (Homalia japonica Besch.) - LTV [244], LAT [3], EST [237], BEL [355], UKR: C [66], M [66], K [66, 328], MLD [367], RUS-EUR: NW [207, 364, 407], NE [207, 212, 415], KLN [309], C [207, 212], N-UR [150, 207, 212], S-UR [150, 207, 212], SE [207], 
CAUC [55, 212, 231, 248], GEO [117], ARM [297], AZE [277], RUS-AS: W-SIB [265], S-SIB [69, 70, 212], E-SIB [212, 275], YAK [239], S-FE [76, 77, 212] - \{105\}

- webbiana (Mont.) Schimp. (H. woronowii Thér.) - GEO $[382,181]-\{106\}$

Homaliadelphus Dixon \& P.de la Varde [*Neckeraceae]

- targionianus (Mitt.) Dixon \& P.de la Varde var. laevidentatus (S.Okamura) Nog. (Homaliadelphus laevidentatus (S.Okamura) Z.Iwats.) - RUS-AS: S-FE [76]

Homalothecium Bruch et al. [Brachytheciaceae], see also Tomentypnum nitens

— aureum (Spruce) H. Rob. - UKR: K [328], KAZ [296], TUR [9, 296]

- laevisetum Sande Lac. - RUS-AS: S-FE [76, 77, 109, 212]

- lutescens (Hedw.) H.Rob. (Camptothecium lutescens (Hedw.) Bruch et al.) - LTV [244], LAT [3], EST [237], UKR: C [66], M [66], K [66, 328], MLD [367], RUS-EUR: NW [207, 291], KLN [309], C [207, 212, 345], S-UR [207], CAUC [55, 212, 232, 248], GEO [117], ARM [297], AZE [277], KAZ [156, 159], TUR [296, 9], KYR [296]

- philippeanum (Spruce) Bruch et al. (H. aristatum Laz.) - UKR: C [66], M [66], K [66, 328], MLD [367], RUS-EUR: KLN [309], SE [207], CAUC [55, 212, 231, 248], GEO [117], ARM [297], AZE [277], KAZ [156, 159, 393], TUR [296], UZB [267], TAD [293, 296], KYR [296, 349, 351], RUSAS: S-SIB [198]

- sericeum (Hedw.) Bruch et al. - LTV [244], LAT [3], EST [237], BEL [355], UKR: C [66], M [66], K [66, 328], MLD [367], RUS-EUR: NW [207, 212, 364, 407], NE [53], KLN [309], C [207, 212, 345], N-UR [150, 207, 415], S-UR [150, 207], SE [207], CAUC [55, 208, 212, 231, 248], GEO [117], ARM [297], AZE [277], KAZ [159, 296, 356], TUR [296], TAD [296], KYR [296, 351]

Homomallium (Schimp.) Loeske [Pylaisiaceae]

- connexum (Cardot) Broth. - RUS-AS: S-SIB [200, 212], S-FE [76, 212, 221]

- incurvatum (Schrad. ex Brid.) Loeske - LTV [244], LAT [3], EST [237], BEL [355], UKR: C [66], M [66], K [66, 328], MLD [367], RUS-EUR: NW [207, 407], C [207, 212, 345], S-UR [150, 207, 212], CAUC [55, 212, 231, 248], GEO [117], ARM [297], AZE [277], KAZ [296], KYR [296], RUS-AS: S-SIB [69, 70, 212], E-SIB [167, 212, 247], S-FE [76, 212]

- japonico-adnatum (Broth.) Broth. - RUS-AS: S-FE [178]

— plagiangium (Müll.Hal.) Broth. - RUS-AS: S-FE [212, 268]

Hondaella Dixon \& Sakurai [*Hypnaceae]

- caperata (Mitt.) Ando, B.C.Tan \& Z.Iwats. (H. brachytheciella (Broth. \& Paris) Ando) - RUS-AS: SFE [76, 212]

Hookeria Sm. [Hookeriaceae]

— acutifolia Hook. \& Grev. - GEO [22, 117]

— lucens (Hedw.) Sm. - UKR: C [66, 413], RUS-EUR: CAUC [55], GEO [117], AZE [277]

Hydrogonium (Müll.Hal.) A.Jaeger [Pottiaceae]

— arcuatum (Griff.) Wijk \& Margad. - TUR [8, 296, 368]

- ehrenbergii (Lorenz) A.Jaeger - KAZ [296, 356], TUR [16, 295, 296], UZB [16], TAD [295], KYR [296]

- mamatkulovii Laz. - TAD [295]

Hydrogrimmia see Grimmia mollis

Hygroamblystegium Loeske [Amblystegiaceae]

- fluviatile (Hedw.) Loeske (Amblystegium fluviatile (Hedw.) Bruch et al.) - LTV [244], LAT [3], EST [237], BEL [355], UKR: C [66], M [66], RUS-EUR: NW [207, 212, 407], KLN [309], C [207], S-UR [150], KAZ [157, 296]

- humile (P.Beauv.) Vanderp., Goffinet \& Hedenäs (Amblystegium humile (P.Beauv.) Crundw., Leptodictyum humile (P.Beauv.) Ochyra, L. kochii (Bruch et al.) Warnst.) - LTV [244], LAT [3], EST [237], BEL [355], UKR: C [66], M [66], RUS-EUR: ARC [47, 150], NW [207, 407], NE [207, 212, 415], KLN [309], C [207, 212, 345], N-UR [150, 207], S-UR [150, 207, 212], SE [207], CAUC [231, 248], GEO [117], ARM [297], AZE [277], TAD [294, 296], KYR [296], RUS-AS: ARC-ESIB [?47], WSIB [265], S-SIB [69, 70, 212], E-SIB [126, 212], ARC-YAK [47, 239], YAK [239], N-FE [94, 140], S-FE [76, 109, 212] 
- tenax (Hedw.) Jenn. (Amblystegium tenax (Hedw.) C.E.O.Jensen) - LTV [244], LAT [3], EST [237], BEL [355], UKR: C [66], M [66], K [66, 328], RUS-EUR: NE [90, 212, 416], KLN [309], C [207, 212, 345], S-UR [150, 207, 212], CAUC [55, 212], GEO [117], ARM [297], AZE [277], KAZ [156, 159, 160, 296], TAD [294, 296], KYR [296, 351], RUS-AS: W-SIB [265], S-SIB [69, 70, 212], YAK [239], S-FE [76, 77, 212]

- varium (Hedw.) Mönk. (Amblystegium varium (Hedw.) Lindb., Orthotheciella varia (Hedw.) Ochyra) LTV [244], LAT [3], EST [237], BEL [355], UKR: C [66], M [66], K [66, 328], MLD [367], RUSEUR: ARC [47, 150], NW [207, 212, 407], NE [207, 415], KLN [309], C [207, 212], N-UR [150, 207], S-UR [150, 207, 212], SE [207, 212], CAUC [208, 212, 231, 248], GEO [117], ARM [297], AZE [277], KAZ [160, 296, 356], TAD [294, 296], KYR [296], RUS-AS: W-SIB [265], S-SIB [69, 70, 212], E-SIB [212, 275], ARC-YAK [239], YAK [239], ARC-FE [38, 40, 212], N-FE [94, 95], S-FE [76]

Hygrohypnella Ignatov \& Ignatova [Scorpidiaceae], see also Ochyraea duriuscula

- bestii (Renauld \& Bryhn) Ignatov \& Ignatova, comb. nov. - Limnobium molle subsp. bestii Renauld \& Bryhn, Bull. Acad. Int. Géogr. Bot. 10: 7. 1901. (Hygrohypnum bestii (Renauld \& Bryhn) Broth.) RUS-AS: N-FE [138, 140], S-FE [67]

- ochracea (Turner ex Wilson) Ignatov \& Ignatova (Hygrohypnum ochraceum (Turner ex Wilson) Loeske) - UKR: C [66], RUS-EUR: ARC [47, 127, 150], NW [138, 273, 364, 407], NE [138, 415], C [138], N-UR [138, 150], S-UR [138, 150], CAUC [55, 138, 231], GEO [117], KAZ [296, 356], TAD [294, 296], RUS-AS: ARC-WSIB [136, 138, 275], W-SIB [138, 265], S-SIB [69, 70, 138], E-SIB [138, 275], ARC-YAK [47, 138, 239], YAK [138, 239], ARC-FE [40, 138], N-FE [138, 140], S-FE [76, 77, $109,138]$

- polare (Lindb.) Ignatov \& Ignatova (Hygrohypnum polare (Lindb.) Loeske, H. polare var. falcatum Broth., H. ehlei (Arnell) Broth.) - RUS-EUR: ARC [47, 138, 150], NW [138, 364], N-UR [150, 415], KAZ [159, 296, 356], TAD [294, 296], KYR [296], RUS-AS: ARC-ESIB [138, 164, 275], W-SIB [138], S-SIB [69, 70, 138], E-SIB [69, 138, 275], ARC-YAK [47, 138, 239], YAK [138, 239], ARC-FE [40, 138], N-FE [94, 138, 141], S-FE [138, 221]

Hygrohypnum Lindb. [Amblystegiaceae], see also Hygrohypnella, Ochyraea, Pseudohygrohypnum

— luridum (Hedw.) Jenn. - LTV [244], LAT [3], EST [237], BEL [355], UKR: C [66], M [66], K [328], MLD [367], RUS-EUR: ARC [47, 127, 138, 150], NW [138, 364, 407], NE [138, 415], KLN [309], C [138, 207, 345], N-UR [138, 150], S-UR [138, 150], CAUC [55, 138, 208, 231, 248], GEO [117], ARM [297], AZE [277], KAZ [156, 296, 356], UZB [347], TAD [296], KYR [296, 349, 351], RUSAS: ARC-WSIB [136], ARC-ESIB [164, 275], W-SIB [265], S-SIB [69, 70, 138], E-SIB [138, 275], ARC-YAK [47, 138, 239], YAK [138, 239], ARC-FE [40, 138], N-FE [138, 140], S-FE [138]

Hylocomiastrum Broth. [Hylocomiaceae]

- pyrenaicum (Spruce) M.Fleisch. (Hylocomium pyrenaicum (Spruce) Lindb.) - UKR: C [66], RUSEUR: ARC [47, 127, 150], NW [212, 273, 364, 407], NE [207, 212, 415], C [207], N-UR [150, 207, 212], S-UR [150, 207, 212], CAUC [55, 212, 231, 248], GEO [117], AZE [277], KAZ [296], RUSAS: ARC-WSIB [136], W-SIB [265], S-SIB [69, 70, 212], E-SIB [212, 275], YAK [239], ARC-FE [40], N-FE [94, 140], S-FE [76, 77, 109, 212]

- umbratum (Hedw.) M.Fleisch. (Hylocomium umbratum (Hedw.) Bruch et al.) - LAT [3], EST [237], UKR: C [66], RUS-EUR: NW [207, 273, 364, 407], NE [207, 212], C [207, 212], N-UR [150, 207, 212, 415], S-UR [150, 207, 212], CAUC [55, 212, 231], GEO [117], AZE [277], KAZ [356], RUSAS: S-SIB [70, 212], S-FE [109]

Hylocomiopsis Cardot [Hylocomiaceae]

— ovicarpa (Besch.) Cardot - RUS-AS: S-FE [76, 109]

Hylocomium Bruch et al. [Hylocomiaceae], see also Hylocomiastrum, Loeskeobryum brevirostre, in older publications Rhytidiadelphus, Pleurozium

- splendens (Hedw.) Bruch et al. - LTV [244], LAT [3], EST [237], BEL [355], UKR: C [66], M [66], K [66, 328], MLD [367], RUS-EUR: ARC [47, 127, 150], NW [207, 212, 364, 407], NE [207, 212, 415], KLN [309], C [207, 212], N-UR [150, 207, 212], S-UR [150, 207, 212], SE [207, 212], CAUC [55, 212, 231, 248], GEO [117], ARM [297], AZE [277], KAZ [156, 296, 356], KYR [296, 351], RUS-AS: ARC-WSIB [136], ARC-ESIB [212], W-SIB [212, 265], S-SIB [69, 70, 212], E-SIB [69, 
212, 275], ARC-YAK [47, 212, 239], YAK [212, 239], ARC-FE [40], N-FE [94, 140], S-FE [76, 77, $109,212]$

- splendens var. obtusifolium (Geh.) Paris - RUS-EUR: ARC [47, 150], N-UR [414], RUS-AS: ARCWSIB [136], ARC-ESIB [164, 212], S-SIB [69, 70, 197, 212], E-SIB [69, 212], ARC-YAK [47, 212, 239], YAK [212, 239], ARC-FE [38, 212], N-FE [140]

Hymenoloma Ochyra [Rhabdoweisiaceae]

- crispulum (Hedw.) Ochyra (Dicranoweisia crispula (Hedw.) Milde) - UKR: C [66], M [66]), RUSEUR: ARC [47, 127, 150], NW [207, 212, 364, 407], NE [207, 212, 415], N-UR [150, 207, 212, 415], S-UR [150, 212], CAUC [55, 102, 212, 231, 248], GEO [117], ARM [297], AZE [277], KAZ [296, 356], TAD [295], KYR [296], RUS-AS: ARC-WSIB [136], ARC-ESIB [164, 212], S-SIB [69, 70, 212], E-SIB [69, 212], ARC-YAK [47, 212, 239], YAK [239], ARC-FE [40, 212], N-FE [94, 140], S$\mathrm{FE}[212,221]-\{107\}$

— intermedium (J.J.Amann) Ochyra (Dicranoweisia intermedia J.J.Amann) - ARM [27, 297], KAZ [27, 296, 356], TAD [294, 295], KYR [27, 296, 351], RUS-AS: ARC-ESIB [164], S-SIB [197, 212], ARC-FE [40, 212], N-FE [40, 140]

Hymenostomum see Weissia

Hymenostylium Brid. [Pottiaceae]

- recurvirostrum (Hedw.) Dixon - LAT [3], EST [237], UKR: C [66], RUS-EUR: ARC [47, 150], NW [207, 364, 407], NE [207, 212, 415], C [381], N-UR [150, 212, 415], S-UR [207], SE [207], CAUC [55, 212, 248], GEO [117], ARM [297], TAD [294, 295], UZB [267], KYR [267, 296, 351], RUSAS: ARC-ESIB [93, 164], S-SIB [69, 70, 212], E-SIB [212, 275], ARC-YAK [47, 239], YAK [239], ARC-FE [40], N-FE [40, 141], S-FE [76, 77, 212]

Hyocomium armoricum (Brid.) Wijk \& Margad. - excluded $-\{108\}$

Hyophila Brid. [Pottiaceae]

— involuta (Hook.) A.Jaeger - RUS-AS: S-SIB [69], S-FE [76]

Hypnum Hedw. [Hypnaceae], see also Stereodon spp., Drepanium recurvatum, Calliergonella lindbergii, Breidleria pratensis

— andoi A.J.E.Sm. (H. mamillatum (Brid.) Loeske) - LTV [244], UKR: C [66], M [66]

- cupressiforme Hedw. - LTV [244], LAT [3], EST [237], BEL [355], UKR: C [66], M [66], K [66, 328], MLD [367], RUS-EUR: ARC [47, 127, 150], NW [207, 212, 364, 407], NE [53, 207, 212, 415], KLN [309], C [207, 212], N-UR [150, 207, 212], S-UR [150, 207, 212], SE [207], CAUC [55, 208, 212, 231, 248], GEO [117], ARM [297], AZE [277], KAZ [156, 296, 356], TUR [296], TAD [296], KYR [296, 351], RUS-AS: ARC-WSIB [136], ARC-ESIB [93, 164, 212], W-SIB [265], S-SIB [69, 212], E-SIB [69, 70, 212, 275], ARC-YAK [47, 212, 239], YAK [212, 239], ARC-FE [40, 212], N-FE [94, 140], S-FE [76, 77, 109, 212]

- cupressiforme var. lacunosum Brid. - LAT [1], EST [237], UKR: 66, K [328], RUS-EUR: KLN [309]

- cupressiforme var. subjulaceum Molendo - UKR [66, 328], MLD [367], RUS-EUR: NW [407], NUR [207], AZE [277], TAD [296], RUS-AS: S-SIB [212, 200], E-SIB [275], ARC-YAK [239], YAK [239], S-FE [212, 221]

- densirameum Ando - excluded - $\{109\}$

— imponens Hedw. - LAT [3], ?BEL [355], UKR: C [66], M [66, 405], K [66, 328], GEO [117], AZE [277], ?KAZ [296], RUS-EUR: CAUC [425], RUS-AS: ?ARC-YAK [47], ?N-FE [94, 140], ?S-FE $[76]-\{110\}$

— jutlandicum Holmen \& E.Warncke - LAT [3], UKR: M [66], RUS-EUR: KLN [309] — \{111\}

- saitoi Ando - RUS-AS: E-SIB [388], YAK [239], N-FE [46], S-FE [212, 221]

Hypopterygium Brid. [Hypopterygiaceae]

— flavolimbatum Müll. Hal. (H. japonicum Mitt.) - RUS-AS: S-FE [76, 77, 212] - $\{112\}$

Indusiella Broth. \& Müll.Hal. [Grimmiaceae]

— thianschanica Broth. \& Müll.Hal. - RUS-EUR: CAUC [12], TAD [295], KYR [103, 296], RUS-AS: S-SIB [69, 70, 212], YAK [239]

Isopterygiopsis Z.Iwats. [Plagiotheciaceae]

— alpicola (Lindb. \& Arnell) Hedenäs (Isopterygium alpicola (Lindb. \& Arnell) Nyholm) - RUS-EUR: 
NW [364], NE [414], RUS-AS: S-SIB [200, 212], E-SIB [163, 275, 388], ARC-YAK [47, 239], YAK [239], ARC-FE [40], N-FE [40, 140], S-FE [212, 221]

- muelleriana (Schimp.) Z.Iwats. (Isopterygium muellerianum (Schimp.) A.Jaeger) - UKR: C [66], RUS-EUR: ARC [47, 127, 150], N-UR [?47], GEO [117], RUS-AS: ARC-WSIB [136], ARC-ESIB [164, 212], S-SIB [69, 70, 212], E-SIB [212, 386], ARC-YAK [239], YAK [239], ARC-FE [40], N-FE $[40,140]$, S-FE $[76,77,212]$

- pulchella (Hedw.) Z.Iwats. (Isopterygium pulchellum (Hedw.) A.Jaeger) - LAT [3], EST [237], UKR: C [66], M [66], RUS-EUR: ARC [47, 127, 150], NW [207, 212, 364, 407], NE [207, 212, 415], C [207], N-UR [150, 207, 212], S-UR [150, 207, 212], CAUC [55, 208, 212, 231, 248], GEO [117], ARM [297], AZE [277], KAZ [159, 296, 356], TAD [296], KYR [296], RUS-AS: ARC-WSIB [136], ARC-ESIB [164, 212], W-SIB [212, 265], S-SIB [69, 70, 212], E-SIB [69, 212, 275], ARC-YAK [47, 212, 239], YAK [212, 239], ARC-FE [40, 212], N-FE [94, 140], S-FE [212, 221]

Isopterygium see Isopterygiopsis, Pseudotaxiphyllum

Isothecium Brid. [Lembophyllaceae], see also Plasteurhynchium striatulum

- alopecuroides (Lam. ex Dubois) Isov. (I. myurum Brid.) - LTV [244], LAT [3], EST [237], BEL [355], UKR: C [66], M [66], K [66, 328], MLD [367], RUS-EUR: NW [207, 212, 364, 407], NE [288], KLN [309], C [207, 212, 345], CAUC [55, 208, 212, 231, 248], GEO [117], ARM [297], AZE [277]

- hakkodense Besch. - RUS-AS: S-FE [77]

- myosuroides Brid. - LAT [3], EST [237], UKR: C [66], RUS-EUR: NW [207, 212, 364, 407], NE [288], KLN [309], CAUC [55, 208, 212, 232], GEO [117]

Iwatsukiella W.R.Buck \& H.A.Crum [*Leskeaceae]

- leucotricha (Mitt.) W.R.Buck \& H.A.Crum - RUS-EUR: N-UR [150, 207, 212], S-UR [150, 207, 212], GEO [372], RUS-AS: S-SIB [69, 70, 212], E-SIB [69, 212], YAK [239], N-FE [40, 94, 140], SFE [76, 77, 109, 212]

Jaffueliobryum Thér. [Grimmiaceae]

— latifolium (Lindb. \& Arnell) Thér. - RUS-EUR: CAUC [249, 250], RUS-AS: W-SIB [339], S-SIB $[69,70,212,275]$, YAK $[239]-\{113\}$

Kiaeria I.Hagen [Rhabdoweisiaceae]

- blyttii (Bruch et al.) Broth. - LAT [3], EST [237], UKR: C [66], RUS-EUR: ARC [47, 150], NW [212, 273, 364, 407], N-UR [150, 207, 415], S-UR [150, 207], CAUC [231], RUS-AS: ARC-ESIB [164], S-SIB [212, 336], E-SIB [163], YAK [239], ARC-FE [40], N-FE [94, 140]

- falcata (Hedw.) I.Hagen - UKR: C [66], RUS-EUR: ARC [47, 150], NW [84, 86], N-UR [150, 415], CAUC [55, 212], GEO [117], AZE [277], RUS-AS: S-SIB [197, 212], N-FE [140], S-FE [67]

- glacialis (Berggr.) I.Hagen - RUS-EUR: ARC [47, 150], NW [212, 273, 364], N-UR [150, 415], RUS-AS: ARC-WSIB [136], ARC-ESIB [212], S-SIB [70], E-SIB [163], ARC-YAK [47, 239], YAK [239], ARC-FE [40, 212], N-FE [94, 140], S-FE [67]

- riparia (H.Lindb.) M.F.V.Corley (Dicranella riparia (H.Lindb.) Mårtensson \& Nyholm) - RUS-EUR: NW [133], RUS-AS: ARC-WSIB [133, 136]

- starkei (F.Weber \& D.Mohr) I.Hagen - UKR: C [66], RUS-EUR: ARC [47, 127, 150, 415], NW [87, 273, 364], N-UR [150, 207, 212, 415], S-UR [150, 207], CAUC [55, 212, 231, 248], GEO [102, 117], AZE [277], RUS-AS: ARC-WSIB [136], S-SIB [70, 212], E-SIB [74, 212], YAK [239], ARC-FE [40, 212], N-FE [140], S-FE [212, 221]

Kindbergia Ochyra [Brachytheciaceae]

- praelonga (Hedw.) Ochyra (Eurhynchium praelongum (Hedw.) Bruch et al., E. praelongum var. stokesii (Turner) Dixon, E. stokesii (Turner) Bruch et al., Oxyrrhynchium praelongum (Hedw.) Warnst.) - LTV [244], LAT [3], EST [237], BEL [355], UKR: C [66], M [66]), RUS-EUR: KLN [309], CAUC [208, 212], GEO [117], AZE [277] - $\{114\}$

Leptobarbula Schimp. [Pottiaceae]

- berica (De Not.) Schimp. - GEO [117, 153], AZE [277]

Leptobryum (Bruch et al.) Wilson [Meesiaceae]

- pyriforme (Hedw.) Wilson - LTV [244], LAT [3], EST [237], BEL [355], UKR: C [66], M [66], MLD [367], RUS-EUR: ARC [47, 127, 150], NW [207, 212, 364, 407], NE [207, 212, 415], KLN 
[309], C [207, 212], N-UR [150, 207, 212], S-UR [150, 207, 212], SE [207, 212], CAUC [208, 212, 248], GEO [117], ARM [297], AZE [277], KAZ [296, 356], TAD [294, 296], KYR [296, 351], UZB [267], RUS-AS: ARC-WSIB [136], ARC-ESIB [164, 212, 275], W-SIB [212, 265], S-SIB [69, 70, 212], E-SIB [69, 212, 275], ARC-YAK [47, 212, 239], YAK [212, 239], ARC-FE [40, 212], N-FE [94, 140], S-FE [77, 109, 212]

Leptodictyum (Schimp.) Warnst. [Amblystegiaceae], see also Hygroamblystegium humile

- riparium (Hedw.) Warnst. (Amblystegium riparium (Hedw.) Bruch et al.) - LTV [244], LAT [3], EST [237], BEL [355], UKR: C [66], M [66], K [328], MLD [367], RUS-EUR: ARC [47, 150], NW [207, 212, 364, 407], NE [207, 212, 415], KLN [309], C [207, 212], N-UR [150, 207, 212], S-UR [150, 207, 212], SE [207, 212], CAUC [55, 212, 232, 248], GEO [117], ARM [297], AZE [277], TUR [296], TAD [296], KYR [296], RUS-AS: ARC-WSIB [136], ARC-ESIB [212], W-SIB [212, 265], S-SIB $[69,70,212]$, E-SIB [212, 275], ARC-YAK [47, 239], YAK [212, 239], ARC-FE [40], N-FE [140], S$\mathrm{FE}[76,109,212]$

Leptodon D.Mohr [Neckeraceae]

— smithii (Hedw.) F.Weber \& D.Mohr - UKR: K [66, 328], RUS-EUR: CAUC [55, 203, 208, 212, 232], GEO [117], AZE [277]

Leptodontium (Müll.Hal.) Lindb. [Pottiaceae]

- flexifolium (Dicks.) Hampe (incl. L. styriacum (Jur.) Limpr.) - RUS-EUR: CAUC [201, 249], RUSAS: S-SIB [69, 70], S-FE [76, 178, 212] - $\{115\}$

Leptopterigynandrum Müll.Hal. [*Hypnaceae] $-\{116\}$

— austro-alpinum Müll.Hal. - RUS-AS: S-SIB [32, 182, 212], ARC-FE [32, 40, 182] - $\{116\}$

- subintegrum (Mitt.) Broth. - RUS-AS: S-SIB [182]

- tenellum Broth. - RUS-AS: S-SIB [182]

Lescuraea Bruch et al. [Pseudoleskeaceae]

- incurvata (Hedw.) E.Lawton (Pseudoleskea incurvata (Hedw.) Loeske) - UKR: C [66], M [66], K [66, 328], RUS-EUR: ARC [47, 127, 150], NW [87, 212, 364], NE [212, 415], N-UR [150, 207, 212, 415], S-UR [150, 207, 212], CAUC [55, 212, 231], GEO [117], ARM [297], AZE [277], KAZ [296, 356], TAD [296], RUS-AS: W-SIB [265], S-SIB [69, 70, 212], ARC-FE [40], N-FE [140], SFE [212, 221]

- incurvata var. gigantea E.Lawton - RUS-EUR: CAUC [231], RUS-AS: ARC-FE [40]

- mutabilis (Brid.) Lindb. - UKR: C [66], RUS-EUR: ?N-UR [415], ?S-UR [150], CAUC [55, 212, 231], GEO [117], ARM [297], AZE [277], KAZ [156, 296, 356], TUR [296], KYR [296], RUS-AS: S-SIB $[212,336]$, ?N-FE $[94,140]-\{117\}$

— patens Lindb. (Pseudoleskea patens (Lindb.) Kindb.) - UKR: C [66], RUS-EUR: NW [364], NE [415], N-UR [150, 415], CAUC [212, 231], AZE [277], KAZ [296], KYR [296], RUS-AS: S-SIB [212, 336], N-FE [140]

- plicata (Schleich. ex F.Weber \& D.Mohr) Broth. (Ptychodium plicatum (Schleich. ex F.Weber \& D.Mohr) Schimp.) - UKR: C [66, 413], RUS-EUR: CAUC [55, 212, 231, 248], GEO [117], ARM [297], AZE [277]

- radicosa (Mitt.) Mönk. (Pseudoleskea radicosa (Mitt.) Macoun \& Kindb.) - UKR: C [66], RUSEUR: ARC [47, 127, 150], NW [87, 273, 364, 407], N-UR [150, 207, 212, 415], S-UR [150, 207, 212], CAUC [55, 212, 231], ARM [297], KAZ [156, 296, 356], RUS-AS: S-SIB [70, 212], E-SIB [212, 247], ?ARC-YAK [47], ARC-FE [40], N-FE [94, 140]

- saviana (De Not.) E.Lawton (Pseudoleskea saviana (De Not.) Latzel) - UKR: C [66], K [66, 328], RUS-EUR: CAUC [55, 231], GEO [117], AZE [277]

— saxicola (Bruch et al.) Molendo - UKR: C [66], RUS-EUR: ARC [47, 127], NW [212, 273, 364, 407], NE [101], N-UR [150, 90, 207, 212], S-UR [150, 207, 212], CAUC [55, 212, 231, 248], GEO [117], AZE [277], KAZ [156, 296, 356], KYR [296], RUS-AS: S-SIB [69, 70, 212], E-SIB [275], YAK [239], ARC-FE [40], N-FE [141], S-FE [76, 109, 212]

- secunda Arnell - RUS-EUR: N-UR [150, 207, 212], RUS-AS: S-SIB [70, 197], N-FE [140]

Leskea Hedw. [Leskeaceae]

— polycarpa Hedw. - LTV [244], LAT [3], EST [237], BEL [355], UKR: C [66], M [66], K [66, 328], 
MLD [367], RUS-EUR: ARC [47, 150], NW [207, 364, 407], NE [207, 212, 415], KLN [309], C [207, 212], N-UR [90, 150, 207], S-UR [150, 207, 212], SE [207], CAUC [55, 212, 232, 248], GEO [117], ARM [297], AZE [277], KAZ [296, 356], RUS-AS: ARC-WSIB [136], ARC-ESIB [?47], W-SIB [212, 265], S-SIB [69, 70, 212], E-SIB [212, 275], YAK [239], N-FE [40, 140], S-FE [76, 77, 109, 212]

Leskeella see Pseudoleskeella

Leucobryum Hampe [Leucobryaceae]

- glaucum (Hedw.) Ångstr. - LTV [244], LAT [3], EST [237], BEL [355], UKR: C [66], M [66], K [328], RUS-EUR: NW [262], KLN [309], C [207, 212, 345], CAUC [55], GEO [117], AZE [277], RUS-AS: S-FE [76, 77, 109, 212]

- juniperoideum (Brid.) Müll.Hal. - UKR [315], RUS-EUR: CAUC [55, 208, 212], GEO [117], RUSAS: S-FE [76, 115, 212]

Leucodon Schwägr. [Leucodontaceae]

- coreensis Cardot - RUS-AS: S-FE [203, 212]

- flagellaris Lindb. ex Broth. - RUS-EUR: CAUC [55, 203, 212, 231], GEO [117]

- immersus Lindb. - RUS-EUR: CAUC [55, 203, 208, 212, 231, 248], GEO [117], ARM [297], AZE [277], TUR [296]

— pendulus Lindb. - RUS-EUR: C [207], RUS-AS: YAK [239], S-FE [76, 114, 203, 212]

- sciuroides (Hedw.) Schwägr. - LTV [244], LAT [3], EST [237], BEL [355], UKR: C [66], M [66], K [66, 328], MLD [367], RUS-EUR: ARC [47, 150], NW [203, 207, 212, 364, 407], KLN [309], C [203, 207, 212], N-UR [150, 203, 207, 212, 415], S-UR [150, 203, 207, 212], SE [207], CAUC [55, 203, 208, 212, 231, 248], GEO [117], ARM [297], AZE [277], KAZ [296, 348, 356], KYR [296], RUS-AS: S-SIB [69, 70, 203, 212], E-SIB [203, 212], YAK [239], S-FE [77, 114, 203, 212]

Limprichtia see Scorpidium

Lindbergia Kindb. [Leskeaceae]

- brachyptera (Mitt.) Kindb. - GEO [117], ARM [297], RUS-AS: S-SIB [20, 69, 212], S-FE [178] $-\{118\}$

— duthiei (Broth.) Broth. - RUS-AS: S-FE [76]

- grandiretis (Lindb. ex Broth.) Ignatov \& Ignatova, comb. nov. - Leskea grandiretis Lindb. ex Broth., Acta Soc. Sci. Fenn. 19(12): 97. 1892 (L. brachyptera auct. Fl. Cauc. non (Mitt.) Kindb.) - RUS-EUR: CAUC [102, 250], GEO [117], ARM [297] - \{118\}

- japonica Cardot - RUS-AS: S-FE [268]

Loeskeobryum M.Fleisch. ex Broth. [Hylocomiaceae]

- brevirostre (Brid.) M.Fleisch. (Hylocomium brevirostre (Brid.) Bruch et al., Loeskeobryum cavifolium M.Fleisch.) - UKR: C [66], K [66, 328], RUS-EUR: KLN [309], C [207], CAUC [14, 232], GEO [117], ARM [297], AZE [277], RUS-AS: N-FE [140], S-FE [109]

Loeskypnum H.K.G.Paul [Calliergonaceae]

- badium (Hartm.) H.K.G.Paul (Drepanocladus badius (Hartm.) G.Roth) - LAT [4], EST [237], RUSEUR: ARC [47, 127, 150], NW [212, 273, 364, 407], NE [207, 212, 416], N-UR [150], RUS-AS: ARC-WSIB [136], ARC-ESIB [164, 275], W-SIB [265], S-SIB [69, 70, 212], E-SIB [212, 275], ARCYAK [47, 212, 239], YAK [212, 239], ARC-FE [40, 212], N-FE [94, 140], S-FE [114, 212, 221]

- wickesii (Grout) Tuom. - RUS-AS: ARC-FE [40]

Lyellia R.Br. [Polytrichaceae]

— aspera (I.Hagen \& C.E.O.Jensen) Frye - RUS-AS: ARC-ESIB [47], E-SIB [212, 398], ARC-YAK [47, 212, 239], YAK [239], ARC-FE [40, 212], N-FE [40, 94], S-FE [212, 221]

Macromitrium Brid. [Orthotrichaceae]

- hymenostomum Mont. - RUS-AS: S-FE [76, 212]

— japonicum Dozy \& Molk. - RUS-AS: S-FE [76, 77, 114, 212]

Mamillariella Laz. [Leskeaceae]

— geniculata Laz. - RUS-AS: S-FE [76, 212]

Meesia Hedw. [Meesiaceae]

— hexasticha (Funck) Bruch - LAT [3], BEL [355], RUS-AS: YAK [239], ARC-FE [40] 
- longiseta Hedw. - LTV [244], LAT [3], EST [237], BEL [355], UKR: M [66], RUS-EUR: NW [207, 212, 364, 407], NE [207, 415], KLN [309], C [207], N-UR [150, 207], S-UR [150, 207], GEO [117], RUS-AS: ARC-WSIB [136], ARC-ESIB [93, 212], W-SIB [212, 265], S-SIB [69, 70], E-SIB $[69,212,275]$, ARC-YAK [47, 212, 239], YAK [212, 239], ARC-FE [40], N-FE [40, 140], S-FE $[76]-\{119\}$

— triquetra (Jolycl.) Ångstr. - LTV [244], LAT [3], EST [237], BEL [355], UKR: C [66], M [66], RUS-EUR: ARC [47, 150], NW [207, 212, 364, 407], NE [207, 212, 415], KLN [309], C [207, 212], N-UR [150, 207], S-UR [150, 207, 212], CAUC [54], GEO [117], ARM [297], AZE [277], RUS-AS: ARC-WSIB [136], ARC-ESIB [164, 212], W-SIB [212, 265], S-SIB [69, 70, 212], E-SIB $[69,212,275]$, ARC-YAK [47, 212, 239], YAK [212, 239], ARC-FE [40, 212], N-FE [94, 140], SFE $[76,212]$

- uliginosa Hedw. - LTV [244], LAT [3], EST [237], BEL [355], UKR: C [66], M [66], RUSEUR: ARC [47, 150], NW [207, 212, 364, 407], NE [207, 212, 415], KLN [309], C [207], N-UR [150, 207, 212], S-UR [150, 207, 212], CAUC [55, 212, 231], GEO [117], ARM [297], AZE [277], KYR [296], RUS-AS: ARC-WSIB [136], ARC-ESIB [164, 212, 275], W-SIB [265], S-SIB [69, 70, 212], E-SIB [212, 275], ARC-YAK [47, 212, 239], YAK [212, 239], ARC-FE [40, 212], N-FE [94, $140], \mathrm{S}-\mathrm{FE}[76,114]$

Metaneckera see Neckera menziesii

Meteorium (Brid.) Dozy \& Molk. [Meteoriaceae]

— buchananii (Brid.) Broth. - RUS-AS: S-FE [278]

Microbryum Schimp. [Pottiaceae] $-\{120\}$

- curvicollum (Hedw.) R.H.Zander (Phascum curvicollum Hedw.) - UKR: M [66], RUS-EUR: KLN [309], SE [207, 212, 377], TUR [11, 296]

- davallianum (Sm.) R.H.Zander (Pottia davalliana (Sm.) C.E.O.Jensen, Tortula angustifolia Lindb. ex Broth.) - LTV [244], LAT [3], EST [237], BEL [355], UKR: C [66], M [66], RUS-EUR: C [207, 345], GEO [117]

— floerkeanum (F.Weber \& D.Mohr) Schimp. (Phascum floerkeanum F.Weber \& D.Mohr) - LTV [244]

- rectum (With.) R.H.Zander (Pottia recta (With.) Mitt.) - CAUCASUS [341, 361]

- starckeanum (Hedw.) R.H.Zander (Pottia starckeana (Hedw.) Müll.Hal.) - UKR: K [66, 328], RUSEUR: KLN [408], TUR [296], TAD [295]

— vlassovii (Laz.) R.H.Zander - ARM [297], AZE [277], KAZ [295], TUR [267, 295, 296], UZB [267, 295, 296], TAD [294, 295], KYR [295, 296, 349, 351]

Mielichhoferia Nees \& Hornsch. [Mielichhoferiaceae], see also Bryum caucasicum (=M. himalayana)

- elongata (Hoppe \& Hornsch. ex Hook.) Hornsch. (M. mielichhoferiana var. elongata (Hoppe \& Hornsch. ex Hook.) Wijk \& Margad.) - TAD [296], RUS-AS: E-SIB [74, 212]

— japonica Besch. (M. mielichhoferiana var. japonica (Besch.) Wijk \& Margad.) - RUS-AS: S-FE [77]

- macrocarpa (Hook.) Bruch \& Schimp. - TAD [296], RUS-AS: S-SIB [69, 70], YAK [239]

- mielichhoferiana (Funck) Loeske - RUS-EUR: CAUC [55, 212, 231], GEO [117], ARM [297], AZE [277], TAD [294, 296], RUS-AS: S-SIB [69, 70], ARC-FE [40], N-FE [141], S-FE [111, 114, 212]

Miyabea Broth. [*Leskeaceae]

- fruticella (Mitt.) Broth. - RUS-AS: S-FE [76]

- rotundifolia Cardot - RUS-AS: S-FE [76]

Mniobryum see Pohlia

Mnium Hedw. [Mniaceae], see also Cyrtomnium, Plagiomnium, Pseudobryum, Rhizomnium, Trachycystis

- blyttii Bruch et al. - RUS-EUR: ARC [47, 150], NW [212, 273, 364], N-UR [150, 415], ?KAZ [296, 356], RUS-AS: ARC-WSIB [136], ARC-ESIB [164, 212], S-SIB [70], E-SIB [126, 212], ARC-YAK [47, 212, 239], YAK [212, 239], ARC-FE [40, 212], N-FE [40]

- heterophyllum (Hook.) Schwägr. - RUS-EUR: C [207, 345], CAUC [248], GEO [117], KAZ [296, 356], KYR [296], RUS-AS: E-SIB [212, 247], S-FE [76, 77, 212]

- hornum Hedw. - LTV [244], LAT [3], EST [237], BEL [355], UKR: C [66], M [66], K [66, 328], RUS-EUR: NW [87, 207, 364, 407], KLN [309], CAUC [55, 212], GEO [31], AZE [277], KAZ [159, 296, 356], KYR [296], RUS-AS: S-FE [77, 109] — \{121\} 
- laevinerve Cardot - TAD [296], KYR [296], RUS-AS: E-SIB [212, 247], N-FE [140], S-FE [76, 77, $212]-\{122\}$

— lycopodioides Schwägr. (M. ambiguum H.Müll., M. magnirete (Lindb. \& Arnell) Kindb.) - LTV [244], BEL [355], UKR: C [66], M [66], RUS-EUR: ARC [47, 127, 150], NW [207, 212, 364, 407], NE [207, 212, 415], KLN [309], C [207, 381], N-UR [150, 207, 212], S-UR [150, 207, 212], CAUC [212, 231, 248], GEO [117], ARM [297], AZE [277], KAZ [296, 356], TAD [296], KYR [296, 351], UZB [267], RUS-AS: ARC-WSIB [136], ARC-ESIB [164, 212], W-SIB [212, 336], S-SIB [69, 70, 212], ESIB [74, 212, 247], ARC-YAK [47, 239], YAK [212, 239], ARC-FE [40, 212], N-FE [94, 140], S-FE $[76,77,109,212]-\{122\}$

- marginatum (Dicks.) P.Beauv. - LTV [244], LAT [3], EST [237], BEL [355], UKR: C [66], M [66], K [66, 328], RUS-EUR: ARC [47, 150], NW [207, 273, 364, 407], NE [207, 212, 415], C [207, 212], N-UR [150, 207, 212], S-UR [150, 207, 212], SE [207], CAUC [55, 212, 231, 248], GEO [117], ARM [297], AZE [277], KAZ [156, 159, 296, 356], TAD [294, 296], KYR [296, 351], RUS-AS: ARCESIB [212], W-SIB [212, 265], S-SIB [69, 70, 212], E-SIB [212, 247], ARC-YAK [47, 239], YAK [212, 239], ARC-FE [40], N-FE [94, 140], S-FE [76, 109, 212]

- spinosum (Voit) Schwägr. - UKR: C [66], K [66, 328], RUS-EUR: ARC [47, 127, 150], NW [87, 273, 364, 407], NE [207, 212, 364, 415], C [207], N-UR [150, 207, 212], S-UR [150, 207, 212], CAUC [55, 212, 231, 248], GEO [117], ARM [297], AZE [277], KAZ [156, 159, 296, 356], TAD [296], KYR [296, 351], RUS-AS: ARC-WSIB [136], W-SIB [212, 265], S-SIB [69, 70, 212], E-SIB [69, 212], ARC-YAK [36, 47, ?239], YAK [239], ARC-FE [40, 212], N-FE [140], S-FE [76, 212, 221]

- spinulosum Bruch et al. - UKR: C [66], RUS-EUR: NE [207, 212, 415], N-UR [150, 207, 212], S-UR [150, 207, 212], CAUC [55, 212, 231, 248], GEO [117], KAZ [296, 356], KYR [296], RUS-AS: WSIB [265], S-SIB [70, 212], E-SIB [212, 247], YAK [239], S-FE [76, 212, 221]

- stellare Hedw. - LTV [244], LAT [3], EST [237], BEL [355], UKR: C [66], M [66], K [66, 328], MLD [367], RUS-EUR: ARC [127, 150], NW [207, 212, 364, 407], NE [207, 212, 415], KLN [309], C [207, 212], N-UR [150, 207, 212], S-UR [150, 207, 212], SE [207], CAUC [55, 208, 212, 231, 248], GEO [117], ARM [297], AZE [277], KAZ [159, 296, 356], KYR [296], RUS-AS: ARC-ESIB [93, 212], W-SIB [212, 265], S-SIB [69, 70, 212], E-SIB [212, 247], ARC-YAK [239], YAK [239], N-FE [140], S-FE [76, 77, 212]

- thomsonii Schimp. - UKR: C [66], K [66, 328], RUS-EUR: ARC [47, 127, 150, 415], NW [364, 407], NE [207, 212, 415], N-UR [150, 207, 212, 415], S-UR [91], CAUC [55, 212, 231, 248], GEO [117], ARM [297], AZE [277], KAZ [156, 159, 296, 356], UZB [347], TAD [294, 296], KYR [296, 349, 351], RUS-AS: ARC-WSIB [136], ARC-ESIB [212], W-SIB [264], S-SIB [69, 70, 212], E-SIB $[69,212]$, ARC-YAK [47, 239], YAK [212, 239], ARC-FE [40, 212], N-FE [94, 141], S-FE [76, 77, 212]

Molendoa Lindb. [Pottiaceae]

- hornschuchiana (Hook.) Lindb. ex Limpr. - UKR: C [66, 413]

- schliephackei (Limpr. ex Schlieph.) R.H.Zander (Anoectangium schliephackei (Limpr. ex Schlieph.) Paris, Pleuroweisia schliephackei Limpr. ex Schlieph.) - RUS-EUR: CAUC [102, 250], GEO [117], TAD [295]

- sendtneriana (Bruch et al.) Limpr. - UKR: C [66], RUS-EUR: ARC [47, 150], C [207, 345], N-UR [150, 207, 212, 415], S-UR [150, 207], CAUC [55, 102, 212], AZE [277], TAD [295], KYR [296], RUS-AS: ARC-ESIB [164], S-SIB [69, 70, 212], E-SIB [74, 212], ARC-YAK [47, 239], YAK [239], ARC-FE [40, 212], N-FE [40], S-FE [76, 212, 221]

- sendtneriana var. transcaspica Györffy - KAZ [105], TUR [296], TAD [295], RUS-AS: S-SIB [69]

- seravschanica Broth. ex Györffy - TAD [295], RUS-AS: ARC-WSIB [136]

- tenuinervis Limpr. - KAZ [296, 356], RUS-AS: ARC-WSIB [136], ARC-ESIB [39], E-SIB [50, 162], ARC-YAK [36, 239], YAK [239], ARC-FE [40], N-FE [40]

Myrinia Schimp. [*Amblystegiaceae]

- pulvinata (Wahlenb.) Schimp. (Pseudoleskea korjakorum Laz.) - LAT [3], EST [237], RUS-EUR: ARC [47, 150], NW [207, 364, 407], NE [207, 212, 415], C [207, 212, 345], N-UR [90, 150, 207, 
212], S-UR [150, 207, 212], RUS-AS: ARC-ESIB [275], W-SIB [212, 265], S-SIB [197, 212], E-SIB [212, 275], ARC-YAK [47, 239], YAK [212, 239], ARC-FE [40], N-FE [40], S-FE [114] - \{123\}

- rotundifolia (Arnell) Broth. (Myuroclada rotundifolia (Arnell) Abramova \& I.I.Abramov) - RUS-AS: E-SIB [162], ARC-YAK [47, 239], YAK [239]

Myurella Bruch et al. [Plagiotheciaceae]

— acuminata Lindb. \& Arnell - RUS-AS: E-SIB [162, 218, 275, 388], YAK [212, 239]

- julacea (Schwägr.) Bruch et al. - LAT [3], EST [237], UKR: C [66], K [66, 328], RUS-EUR: ARC [47, 150], NW [212, 364, 407], NE [207, 212, 415], C [207], N-UR [150, 207, 212], S-UR [150, 207, 212], SE [207], CAUC [55, 212, 231, 248], GEO [117], ARM [297], AZE [277], KAZ [156, 296, 356], TAD [294, 296], KYR [296], RUS-AS: ARC-WSIB [136], ARC-ESIB [164, 212, 275], W-SIB [265], S-SIB [69, 70, 212], E-SIB [69, 212, 275], ARC-YAK [47, 212, 239], YAK [212, 239], ARC-FE $[40,212]$, N-FE [94, 140], S-FE [114, 212, 221]

- sibirica (Müll.Hal.) Reimers - RUS-EUR: ARC [47, 150], N-UR [150, 207, 415], S-UR [150], CAUC [249], RUS-AS: W-SIB [265], S-SIB [69, 70, 212], E-SIB [69, 162, 212, 275], ARC-YAK [47, 239], YAK [212, 239], ARC-FE [40], N-FE [140], S-FE [76, 212, 221]

- tenerrima (Brid.) Lindb. - UKR: C [66], RUS-EUR: ARC [47, 150], NW [87, 212, 364, 407], N-UR [150, 207, 212, 415], CAUC [14], GEO [13], AZE [277], KAZ [296, 356], TUR [296], RUS-AS: ARC-WSIB [136], ARC-ESIB [164, 212, 275], S-SIB [69, 70, 212], E-SIB [212, 275], ARC-YAK $[47,239]$, YAK [212, 239], ARC-FE [40, 212], N-FE [94, 140] - $\{124\}$

Myuroclada Besch. [Brachytheciaceae], see also Myrinia rotundifolia

- maximowiczii (G.G.Borshch.) Steere \& W.B.Schofield - RUS-EUR: SE [207, 404], RUS-AS: W-SIB [265], S-SIB [69, 70, 198, 212], E-SIB [69, 212, 275], YAK [212, 239], N-FE [94, 140], S-FE [76, 77, $109,212]-\{125\}$

Neckera Hedw. [Neckeraceae]

- besseri (Lobarz.) Jur. (Homalia besseri Lobarz.) - UKR: C [66], M [66, 148], K [66, 328], MLD [367], RUS-EUR: NW [407], N-UR [150, 207, 415], S-UR [150, 207, 212], SE [207], CAUC [55, 208, 212, 231], GEO [117], ARM [297], AZE [277], KAZ [296, 356], RUS-AS: S-SIB [70, 212]

- borealis Nog. - RUS-AS: S-SIB [69, 70, 212], E-SIB [74, 212, 247], S-FE [76, 77, 109, 212]

- complanata (Hedw.) Huebener - LTV [244], LAT [3], EST [237], BEL [355], UKR: C [66], M [66], K [66, 328], RUS-EUR: NW [207, 364, 407], NE [53], KLN [309], C [207], N-UR [150, 207, 212, 415], S-UR [150, 207, 212], CAUC [55, 208, 212, 231, 248], GEO [117], ARM [297], AZE [277], ?KAZ [156, 159, 296]

- crispa Hedw. - LTV [244], LAT [3], EST [237], BEL [?355], UKR: C [66], M [66], K [66, 328], RUS-EUR: NW [407], KLN [309], C [?207], CAUC [55, 208, 212, 231, 248], GEO [117], ARM [297], AZE [277]

- menziesii Drumm. (Metaneckera menziesii (Drumm.) Steere, Neckera turgida Jur.) - UKR: K [66, 328, 341], GEO [28, 117]

— pennata Hedw. - LTV [244], LAT [3], EST [237], BEL [355], UKR: C [66], M [66], K [66, 328], MLD [367], RUS-EUR: ARC [47, 150], NW [207, 212, 364, 407], NE [207, 212, 415], KLN [309], C [207, 212, 345], N-UR [150, 207, 212], S-UR [150, 207, 212], SE [207], CAUC [55, 212], GEO [117], ARM [297], AZE [277], KAZ [156, 296, 356], KYR [296], RUS-AS: ARC-WSIB [136], ARC-ESIB [164, 212], W-SIB [265], S-SIB [69, 70, 212], E-SIB [69, 212, 275], ARC-YAK [47], YAK [212, 239], ARC-FE [40], N-FE [140], S-FE [76, 77, 212]

- pennata var. tenera Müll.Hal. (N. oligocarpa Bruch) - RUS-EUR: ARC [127, 150], NW [85, 212, 364, 407], NE [415], N-UR [150, 415], S-UR [150, 212], RUS-AS: S-SIB [69, 70, 212], E-SIB [212, 275], ARC-YAK [239], YAK [239], N-FE [94], S-FE [113, 212]

— pumila Hedw. - UKR: C [66], RUS-EUR: CAUC [55, 208, 212], GEO [117], ARM [297]

- yezoana Besch. - RUS-AS: S-FE [76, 77, 109, 212]

?Neckeropsis nitidula (Mitt.) M.Fleisch. - RUS-AS: S-FE [376] $-\{52\}$

Niphotrichum (Bednarek-Ochyra) Bednarek-Ochyra \& Ochyra [Grimmiaceae]

— barbuloides (Cardot) Bednarek-Ochyra \& Ochyra (Racomitrium barbuloides Cardot) - RUS-AS: NFE [140] 
- canescens (Hedw.) Bednarek-Ochyra \& Ochyra (Racomitrium canescens (Hedw.) Brid.) - LTV [244], LAT [3], EST [237], BEL [355], UKR: C [66], M [66], K [66, 328], RUS-EUR: ARC [47, 127, 150], NW [207, 212, 364, 407], NE [207, 212, 415], KLN [309], C [207, 212], N-UR [150, 207, 212], S-UR [150, 207, 212], SE [207], CAUC [55, 212, 231, 248], GEO [117], ARM [297], AZE [277], KAZ [296, 356], KYR [296], RUS-AS: ARC-WSIB [136], ARC-ESIB [96, 212], S-SIB [69, 70, 202, 212], E-SIB [69, 212, 275], ARC-YAK [47, 212, 239], YAK [212, 239], ARC-FE [40, 212], N-FE [94, 140], S-FE [76, 77, 212]

- elongatum (Frisvoll) Bednarek-Ochyra \& Ochyra (Racomitrium elongatum Ehrh. ex Frisvoll) - EST [237], UKR: C [402], K [328, 402], RUS-EUR: KLN [309]

- ericoides (Brid.) Bednarek-Ochyra \& Ochyra (Racomitrium ericoides (F.Weber ex Brid.) Brid.) - LAT [3], EST [237], UKR [66], RUS-EUR: ARC [47, 150], NW [364, 407], KLN [309], CAUC [102], GEO [117], RUS-AS: ARC-ESIB [50], ARC-YAK [47, 239], YAK [239], ARC-FE [40, 212], N-FE $[40,140]$

— japonicum (Dozy \& Molk.) Bednarek-Ochyra \& Ochyra (Racomitrium japonicum Dozy \& Molk.) RUS-AS: S-FE [80, 114]

- muticum (Kindb.) Bednarek-Ochyra \& Ochyra (Racomitrium muticum (Kindb.) Frisvoll) - RUS-AS: N-FE [140], S-FE [67]

— panschii (Müll.Hal.) Bednarek-Ochyra \& Ochyra (Racomitrium panschii (Müll.Hal.) Kindb.) - RUSEUR: ARC [47], RUS-AS: ARC-ESIB [164], S-SIB [202, 212], E-SIB [163], ARC-YAK [47, 239], YAK [117, 239], ARC-FE [40, 212], N-FE [140], S-FE [212, 221]

Nyholmiella see Orthotrichum

Ochyraea Váňa [*Amblystegiaceae]

- alpestris (Hedw.) Ignatov \& Ignatova (Hygrohypnum alpestre (Hedw.) Loeske) - RUS-EUR: ARC [47, 138, 150], NW [138, 273, 364, 407], NE [207, 415], N-UR [150, 138, 415], S-UR [138, 150], CAUC [138], KAZ [296, 159], RUS-AS: ARC-WSIB [136], ARC-ESIB [138, 164, 275], W-SIB [138], S-SIB [138], E-SIB [138], ARC-YAK [47, 138, 239], YAK [138, 239], ARC-FE [40, 138], N-FE [40]

- cochlearifolia (Venturi) Ignatov \& Ignatova (Hygrohypnum cochlearifolium (Venturi) Broth.) - RUSEUR: NW [138, 364], N-UR [138, 150, 207], CAUC [138], RUS-AS: ARC-WSIB [136], W-SIB [138], S-SIB [138], E-SIB [138], ARC-FE [40, 138], N-FE [138, 140], S-FE [138, 221]

- duriuscula (De Not.) Ignatov \& Ignatova, comb. nov. - Limnobium duriusculum De Not., Erbario Crittogamico Italiano ser. 2, 204. 1869. (Hygrohypnum duriusculum (De Not.) D.W.Jamieson) - UKR: C [66], RUS-EUR: ARC [47, 127, 150], NW [138, 273, 364, 407], NE [138], N-UR [90, 138, 150, 415], S-UR [138, 150], CAUC [55, 138, 231, 248], GEO [117], KAZ [156, 159], KYR [296], RUSAS: ARC-WSIB [136], S-SIB [69, 70, 138], E-SIB [138, 167, 275], YAK [138, 239], ARC-FE [40, 138], N-FE [138, 140], S-FE [76, 114, 138]

- mollis (Hedw.) Ignatov (Hygrohypnum molle (Hedw.) Loeske) - LTV [244], UKR: C [66], RUS-EUR: NW [87, 138, 364], ARM [297], RUS-AS: YAK [138, 239], ARC-FE [40, 138], N-FE [138, 221]

- norvegica (Bruch et al.) Ignatov \& Ignatova (Hygrohypnum norvegicum (Bruch et al.) J.J.Amann) RUS-EUR: NW [138, 364], N-UR [150, 415], RUS-AS: ARC-ESIB, S-SIB [138], E-SIB [138, 275], N-FE [138], S-FE [138, 221]

- smithii (Sw.) Ignatov \& Ignatova (Hygrohypnum smithii (Sw.) Broth.) - RUS-EUR: NW [87, 100, 138, 364], CAUC [138], GEO [15], ARM [297], TAD [296], RUS-AS: S-FE [67]

Oedipodium Schwägr. [Oedipodiaceae]

— griffithianum (Dicks.) Schwägr. - RUS-AS: S-FE [209]

Okamuraea Broth. [Brachytheciaceae]

- brachydictyon (Cardot) Nog. - RUS-AS: S-FE [76, 77, 212]

- hakoniensis (Mitt.) Broth. - RUS-AS: S-FE [76, 109, 212]

Oligotrichum DC. [Polytrichaceae]

- aligerum Mitt. - RUS-AS: N-FE [140], S-FE [76, 77, 114]

- falcatum Steere - RUS-AS: S-SIB [238], ARC-YAK [47, 212, 238, 239], YAK [238, 239], ARC-FE [40, 238], N-FE [238], S-FE [212, 238] 
- hercynicum (Hedw.) Lam. \& DC. - UKR: C [66], RUS-EUR: ARC [47, 127, 150, 238], NW [212, 238, 273, 291, 364], NE [417a], C [207, 238], N-UR [150, 207, 212, 238], S-UR [150], CAUC [55, 212, 231, 238, 248], GEO [117], RUS-AS: ARC-ESIB [47], S-SIB [212, 238], E-SIB [126, 212], ARC-FE [40], N-FE [140, 238], S-FE [77, 212]

— parallelum (Mitt.) Kindb. - RUS-AS: N-FE [94, 140], S-FE [76, 114, 212]

Oncophorus (Brid.) Brid. [Rhabdoweisiaceae]

- compactus (Bruch et al.) Kindb. (O. wahlenbergii var. compactus (Bruch et al.) Braithw.) - RUSEUR: ARC [47, 150], NW [212, 273, 364, 407], N-UR [150], RUS-AS: ARC-WSIB [136], ARCESIB [39, 164], E-SIB [126, 212, 386], ARC-YAK [47], YAK [239], ARC-FE [40, 212], N-FE [40, 140]

- crispifolius (Mitt.) Lindb. - ?KAZ [296, 356], RUS-AS: N-FE [140], S-FE [76, 109, 212] - \{61\}

- virens (Hedw.) Brid. - UKR: C [66], RUS-EUR: ARC [47, 127, 150], NW [212, 273, 364, 407], NE [207, 212, 415], N-UR [150, 207, 212], S-UR [150, 207], CAUC [55, 212, 231, 248], GEO [117], ARM [297], AZE [277], KAZ [156],TAD [294, 295], KYR [296, 351], RUS-AS: ARC-WSIB [136], ARC-ESIB [164, 212, 275], W-SIB [265], S-SIB [69, 70, 212], E-SIB [69, 212, 275], ARC-YAK [47, 239], YAK [239], ARC-FE [40, 212], N-FE [94, 140], S-FE [76, 109, 212]

- wahlenbergii Brid. - LAT [3], EST [237], UKR: C [66], RUS-EUR: ARC [47, 127, 150], NW [207, 212, 364, 407], NE [207, 212, 415], C [207], N-UR [90, 150, 207, 212], S-UR [150, 207], ARM [297], KAZ [156, 296, 356], KYR [296, 351], RUS-AS: ARC-WSIB [136], ARC-ESIB [164, 212, 275], WSIB [212, 265], S-SIB [69, 70, 212], E-SIB [69, 212, 275], ARC-YAK [47, 212, 239], YAK [212, 239], ARC-FE [40, 212], N-FE [94, 140], S-FE [76, 77, 109, 212]

Oreas Brid. [Rhabdoweisiaceae]

- martiana (Hoppe \& Hornsch.) Brid. - RUS-EUR: CAUC [102, 212, 248], GEO [102], RUS-AS: SSIB [69, 70], ARC-FE [40]

Orthodicranum see Dicranum

Orthodontium Schwägr. [Orthodontiaceae]

- lineare Schwägr. (O. australe Hook.f. \& Wilson) - RUS-EUR: KLN [309]

Orthodontopsis Ignatov \& B.C.Tan [Orthodontiaceae]

- bardunovii Ignatov \& B.C.Tan - RUS-AS: S-SIB [212, 220, 334]

Orthothecium Bruch et al. [Plagiotheciaceae]

— chryseon (Schwägr.) Bruch et al. - RUS-EUR: ARC [47, 150], NW [364, 407], N-UR [150, 415], ARM [297], RUS-AS: ARC-WSIB [136], ARC-ESIB [164, 212], W-SIB [47], S-SIB [69, 70, 212], ESIB [74, 212], ARC-YAK [47, 212, 239], YAK [239], ARC-FE [40, 212], N-FE [94, 140], S-FE [114, 212, 221]

- intricatum (Hartm.) Bruch et al. - UKR: C [66], K [66, 328], RUS-EUR: ARC [47], NE [212, 415], N-UR [150, 207], S-UR [150, 207], CAUC [55, 208, 212, 231], GEO [117], ARM [297], AZE [277], KAZ [159],TAD [296], KYR [296], RUS-AS: ARC-WSIB [136], S-SIB [70, 212], YAK [239]

- rufescens (Dicks. ex Brid.) Bruch et al. - UKR: C [66], RUS-EUR: NW [407], ?NE [415], ?N-UR [415], CAUC [55, 212] - $\{126\}$

- strictum Lorentz - RUS-EUR: ARC [47], NW [364, 407], NE [207, 212, 415], N-UR [150, 207, 212 , 415], S-UR [91], RUS-AS: ARC-WSIB [136], ARC-ESIB [164, 212], S-SIB [69, 70, 212], E-SIB [212, 275], ARC-YAK [47, 239], YAK [212, 239], ARC-FE [40, 212], N-FE [94], S-FE [114]

Orthotrichum Hedw. [Orthotrichaceae]

— affine Brid. (O. fastigiatum Bruch ex Brid.) - LTV [244], LAT [3], EST [237], BEL [355], UKR: C [66], M [66], K [66, 328], MLD [367], RUS-EUR: NW [85, 207, 212], KLN [309], C [207, 212, 345], S-UR [150, 207], SE [207], CAUC [208, 212, 232, 248], GEO [117], ARM [297], AZE [277], KAZ [156, 348], TUR [296], TAD [296], RUS-AS: ?N-FE [140] - \{127\}

- alpestre Hornsch. ex Bruch et al. - UKR: C [66], RUS-EUR: NW [212, 364, 407], S-UR [150, 207, 212], CAUC [231, 248], GEO [117], ARM [297], KAZ [157, 296, 356], TAD [294, 296], KYR [267, 296], RUS-AS: ARC-WSIB [136], S-SIB [70, 212, 214], E-SIB [275]

— anomalum Hedw. - LTV [244], LAT [3], EST [237], BEL [355], UKR: C [66], M [66], K [66, 328], MLD [367], RUS-EUR: ARC [47, 150, 415], NW [207, 364, 407], NE [207, 212, 415], KLN [309], C 
[207, 212], N-UR [90, 150, 207, 212], S-UR [150, 207, 212], SE [207], CAUC [55, 208, 212, 231, 248], GEO [117], ARM [297], AZE [277], KAZ [159, 296, 356], TUR [296], TAD [294, 296], KYR [267, 296, 351], UZB [267], RUS-AS: ARC-WSIB [136], S-SIB [69, 70, 212, 214], E-SIB [162], ARC-YAK [47, 239], YAK [212, 239], ARC-FE [40], N-FE [40], S-FE [114, 212, 268]

- callistomum Fisch.-Oost. ex Bruch et al. - RUS-EUR: CAUC [57, 212, 248]

- consobrinum Cardot - RUS-AS: S-FE [76, 212]

- cupulatum Brid. (O. limprichtii I.Hagen) - LAT [3], EST [237], BEL [355], UKR: C [66], M [66], K [66, 328], MLD [367], RUS-EUR: NW [407], C [207, 212, 345], N-UR [90, 150, 207, 212], S-UR [150], SE [207], CAUC [212, 231], GEO [117], ARM [297], AZE [277], TUR [267, 296], TAD [294, 296], KYR [296, 351], RUS-AS: S-SIB [212, 214]

- cupulatum var. riparium Huebener (O. cupulatum var. nudum (Dicks.) Braithw., O. nudum Dicks.) UKR: M [66], K [66, 328], RUS-EUR: KLN [309], TUR [296]

- dasymitrium Lewinsky - RUS-AS: S-SIB [214]

- diaphanum Brid. - LTV [244], LAT [3], EST [237], BEL [355], UKR: C [66], M [66], K [66, 328], MLD [367], RUS-EUR: NW [261], KLN [309], SE [207], CAUC [212, 232, 248], GEO [117], ARM [297], AZE [277], KYR [350],

- furcatum Otnyukova - RUS-AS: S-SIB [212, 321]

- gymnostomum Bruch ex Brid. - LAT [3], EST [237], BEL [355], UKR: C [66], M [66, 402], K [66, 328], MLD [367], RUS-EUR: NW [207, 407], NE [207], C [207, 212], CAUC [55, 212, 231], AZE [277], KAZ [296, 356], RUS-AS: S-SIB [212, 395], YAK [239]

- hallii Sull. \& Lesq. - KAZ [272]

- holmenii Lewinsky-Haapasaari - KAZ [272]

- iwatsukii Ignatov (O. laevigatum J.E.Zetterst. var. japonicum (Z.Iwats.) Lewinsky) - KAZ [155], RUSAS: ARC-ESIB [164, 212], S-SIB [212, 214], E-SIB [163], YAK [239] - $\{128\}$

- laevigatum J.E.Zetterst. - RUS-EUR: NE [415], KAZ [155, 160, 296, 356], TAD [294, 296], RUSAS: ARC-ESIB [212], ARC-YAK [239], YAK [239], ARC-FE [40], N-FE [40] - $\{128\}$

- lyellii Hook. \& Taylor - LTV [244], LAT [3], EST [396], BEL [355], UKR: C [66], M [66], MLD [367], RUS-EUR: KLN [309], CAUC [102, 208, 212, 231], GEO [117], AZE [277], KAZ [296, 356], TAD [296]

- microcarpum De Not. - GEO [117]

- obtusifolium Brid. - LTV [244], LAT [3], EST [237], BEL [355], UKR: C [66], M [66], K [66, 328], MLD [367], RUS-EUR: NW [207, 212, 364, 407], NE [207, 212, 415], KLN [309], C [207, 212], NUR [150, 207, 212], S-UR [150, 207, 212], SE [207, 212], CAUC [55, 212, 231, 248], GEO [117], ARM [297], AZE [277], KAZ [160, 348], TUR [296], TAD [293, 296], KYR [296], RUS-AS: WSIB [212, 265, 275], S-SIB [212, 69, 70, 214], E-SIB [69, 212, 275], YAK [212, 239], ARC-FE [40], N-FE [140], S-FE [76, 77, 114, 212]

— pallens Bruch ex Brid. (O. sibiricum Gronvall) - LAT [3], EST [237], BEL [355], UKR: C [66], M [66], K [66, 328], MLD [367], RUS-EUR: NW [207, 391, 407], KLN [309], C [207, 212, 345], S-UR [91, 207, 212], SE [207], CAUC [55, 212, 231, 248], GEO [117], ARM [297], AZE [277], KAZ [356], TUR [296], TAD [294], UZB [267], RUS-AS: ARC-ESIB [164, 212, 275], S-SIB [212, 214, 275], E-SIB [212, 275], YAK [239], N-FE [40] - \{129\}

— patens Bruch ex Brid. - LAT [3], BEL [355], UKR: C [66], M [66], MLD [367], RUS-EUR: KLN [309], CAUC [208, 212], ARM [297], AZE [277], KAZ [348], TAD [294, 296], KYR [296]

- pellucidum Lindb. - RUS-AS: S-SIB [212, 214], N-FE [40]

- pumilum Sw. ex anon. (O. fallax Bruch ex Brid., O. schimperi Hammar, O. philibertii Venturi) - LTV [244], LAT [3], EST [237], BEL [355], UKR: C [66], M [66], K [66, 328], MLD [367], RUS-EUR: NW [207], KLN [309], C [207, 212, 345], S-UR [150, 207], SE [207, 212], CAUC [55, 208, 212, 231, 248], GEO [117], ARM [297], AZE [277], KAZ [156, 159, 296, 348, 356], TUR [267, 296], TAD [293, 294, 296], KYR [267, 296, 351], RUS-AS: S-SIB [70, 212, 214]

- pylaisii Brid. - RUS-EUR: ARC [47], NW [85, 212, 261, 287, 364], RUS-AS: N-FE [140]

- rogeri Brid. - GEO [102], AZE [277], RUS-AS: S-SIB [212, 214]

- rupestre Schleich. ex Schwägr. - LAT [3], EST [237], UKR: C [66], M [66], K [66, 328], RUS-EUR: 
NW [364, 407], S-UR [150, 207], CAUC [55, 212, 231, 248], GEO [117], ARM [297], AZE [277], KAZ [156, 159, 296, 356], TUR [296], TAD [294, 296], KYR [296, 351], RUS-AS: S-SIB [69, 70, 212, 214], S-FE [76]

— rupestre var. sturmii (Hornsch.) Jur. - TAD [296], KYR [267, 296]

- scanicum Gronvall - UKR: M [66], RUS-EUR: CAUC [231], RUS-AS: E-SIB [212, 247]

- sordidum Sull. \& Lesq. (O. caucasicum Venturi) - RUS-EUR: CAUC [55, 57, 102, 212, 231, 248], ARM [297], KYR [296], RUS-AS: S-SIB [212, 214], E-SIB [212, 247], YAK [239], ARC-FE [40, 212], N-FE [140], S-FE [76, 77, 212]

— speciosum Nees (O. killiasii Müll.Hal., O. elegans Schwägr. ex Hook. \& Grev.) - LTV [244], LAT [3], EST [237], BEL [355], UKR: C [66], M [66], K [66, 328], MLD [367], RUS-EUR: ARC [47, 127, 150], NW [207, 212, 364, 407], NE [207, 212, 415], KLN [309], C [207, 212], N-UR [150, 207, 212], S-UR [150, 207, 212], SE [207, 212], CAUC [55, 212, 231, 248], GEO [117], ARM [297], AZE [277], KAZ [296, 356, 348], TUR [296], TAD [294, 296], KYR [296, 351], RUS-AS: ARC-WSIB [136], ARC-ESIB [212], W-SIB [212, 265], S-SIB [69, 70, 212, 214], E-SIB [69, 212], ARC-YAK [47, 239], YAK [212, 239], ARC-FE [40, 212], N-FE [94, 140], S-FE [212, 268, 221]

- sprucei Mont. - KAZ [175]

- stellatum Brid. - excluded - $\{130\}$

- stramineum Hornsch. ex Brid. - EST [237], BEL [355], UKR: C [66], M [66], K [66, 328], RUSEUR: KLN [309], CAUC [55, 208, 212, 231], GEO [117], ARM [297], AZE [277], KAZ [157]

- striatum Hedw. - LTV [244], LAT [3], BEL [355], UKR: C [66], M [66], K [66, 328], MLD [367], RUS-EUR: KLN [309], CAUC [55, 208, 212, 231, 248], GEO [117], ARM [297], AZE [277], KAZ [348], TUR [296], KYR [296], RUS-AS: S-SIB [212, 214], S-FE [110, 212, 221]

- tenellum Bruch ex Brid. (O. australe Jur.) - BEL [355], UKR: C [66], M [66], K [66, 328], ARM [297], KAZ [348, 393], TAD [296], KYR [296], UZB [267]

- urnaceum Müll.Hal. - ARM [102, 297], AZE [277]

- urnigerum Myrin - RUS-EUR: NW [407], CAUC [102, 231], AZE [277], KYR [267], RUS-AS: ARC-FE [40]

- vicarium Laz. - KYR [267, 271, 296]

— vladikavkanum Venturi - RUS-EUR: CAUC [55, 57, 212, 248], RUS-AS: S-SIB [212, 214]

Oxyrrhynchium (Bruch et al.) Warnst. [Brachytheciaceae]

- hians (Hedw.) Loeske (Eurhynchium hians (Hedw.) Sande Lac.) - LTV [244], LAT [3], EST [237], BEL [355], UKR: C [66], M [66], K [66, 328], MLD [367], RUS-EUR: NW [207, 291], NE [207, 416], KLN [309], C [207, 212], N-UR [207], S-UR [150, 207, 212], SE [207, 212], CAUC [55, 208, 212, 231, 248], GEO [117], ARM [297], AZE [277], KAZ [156], TAD [296], KYR [267, 296], RUSAS: W-SIB [265], S-SIB [69, 70, 198, 212], E-SIB [212, 275], ARC-YAK [47], YAK [239], N-FE [140], S-FE [76, 77, 109, 212] - $\{131\}$

- pumilum (Wilson) Loeske (Eurhynchium pumilum (Wilson) Schimp., Rhynchostegiella pumila (Wilson) E.F.Warb.) - RUS-EUR: CAUC [208], AZE [277]

- savatieri (Schimp. ex Besch.) Broth. - RUS-AS: S-FE [178]

- schleicheri (R.Hedw.) Röll (Eurhynchium schleicheri (R.Hedw.) Milde) - LTV [244], LAT [3], UKR: K [66, 328], MLD [21], RUS-EUR: KLN [309], CAUC [208, 212], ARM [297], AZE [277], ?KAZ [70, 296], ?RUS-AS: S-FE [76, 268] - $\{132\}$

- speciosum (Brid.) Warnst. (Eurhynchium speciosum (Brid.) Jur.) - BEL [355], UKR: C [66], M [66], K [66, 328], RUS-EUR: KLN [309], S-UR [207], GEO [117], ARM [297], AZE [277], TUR [9, 296], UZB [347], KYR [296] - \{133\}

Oxystegus (Lindb. ex Limpr.) Hilp. [Pottiaceae]

- tenuirostris (Hook. \& Taylor) A.J.E.Sm. (Trichostomum tenuirostre (Hook. \& Taylor) Lindb.) LAT [3], EST [397], BEL [355], UKR: C [66], M [66], RUS-EUR: NW [207, 212, 364, 407], C [207], N-UR [150, 207, 212], S-UR [150, 207, 212], CAUC [55, 208, 212, 231, 248], GEO [117], ARM [297], AZE [277], KAZ [157, 296], TAD [294, 295], KYR [296], RUS-AS: ARC-ESIB [96, 212], S-SIB [69, 70, 212], E-SIB [212, 386], YAK [239], ARC-FE [40], N-FE [94, 140], S-FE [76, 77, 109, 212] 
Palamocladium Müll.Hal. [Brachytheciaceae]

— euchloron (Müll.Hal.) Wijk \& Margad. - UKR: K [66, 328], RUS-EUR: ?SE [207], CAUC [55, 208, 212, 232, 250], GEO [117], ARM [297], AZE [277], TUR [9, 296]

Paludella Brid. [Meesiaceae]

- squarrosa (Hedw.) Brid. - LTV [244], LAT [3], EST [237], BEL [355], UKR: M [66], RUS-EUR: ARC [47, 150], NW [207, 212, 364, 407], NE [207, 212, 415], KLN [309], C [207], N-UR [150, 207, 212], S-UR [150, 207, 212], CAUC [212, 231, 248], GEO [117], ARM [297], AZE [277], KAZ [296, 303, 356], RUS-AS: ARC-WSIB [136], ARC-ESIB [212], W-SIB [212, 265], S-SIB [69, 70, 212], E-SIB [69, 212 , 275], ARC-YAK [47, 212, 239], YAK [212, 239], ARC-FE [40], N-FE [94, 140], S-FE [67, 212]

Palustriella Ochyra [Amblystegiaceae]

- commutata (Hedw.) Ochyra (Cratoneuron commutatum (Hedw.) G.Roth) - LTV [244], LAT [3], EST [237], BEL [355], UKR: C [66], M [66], K [66, 328], RUS-EUR: ARC [47, 150], NW [207, 212, 364, 407], NE [366a], KLN [309], C [207, 212, 345], N-UR [150, 207, 212, 415], S-UR [150, 207, 212], CAUC [55, 212, 231, 248], GEO [117], ARM [297], AZE [277], KAZ [156, 296, 356], TUR [267, 296], UZB [267], TAD [294, 296], KYR [267, 296, 351], RUS-AS: W-SIB [265], S-SIB [69, 70, 212], E-SIB [212, 247], YAK [212], N-FE [40, 140], S-FE [37, 212]

- decipiens (De Not.) Ochyra (Cratoneuron decipiens (De Not.) Loeske) - LAT [5], EST [237], BEL [355], UKR: C [66], RUS-EUR: NW [207, 212, 364, 407], NE [207, 212, 415], C [207], N-UR [150, 415], S-UR [150, 207, 212], CAUC [55, 212, 231, 248], GEO [117], ARM [297], AZE [277], KAZ [156, 296, 356], TAD [294, 296], KYR [296, 351], RUS-AS: S-SIB [69, 70, 212], E-SIB [212, 247], YAK [212], S-FE [114]

- falcata (Brid.) Hedenäs (Cratoneuron falcatum (Brid.) G.Roth, P. commutata var.falcata (Brid.) Ochyra) - LAT [1], EST [237], UKR: [66], C [269], RUS-EUR: NW [87, 212, 364, 407], NE [414], AZE [277], TUR [296], TAD [296], KYR [296], RUS-AS: S-SIB [70] $-\{134\}$

Paraleucobryum (Limpr.) Loeske [Dicranaceae]

- enerve (Thed.) Loeske - UKR: C [66], RUS-EUR: ARC [47, 150], NW [87, 212, 364], N-UR [150, 415], CAUC [55, 212, 231, 248], GEO [117], AZE [277], KAZ [296], RUS-AS: S-SIB [69, 70, 212], E-SIB [212, 247], ARC-FE [40], N-FE [140], S-FE [76, 212]

- longifolium (Hedw.) Loeske - LTV [244], LAT [3], EST [237], BEL [355], UKR: C [66], M [66]), RUS-EUR: ARC [47, 150], NW [207, 212, 364, 407], NE [212, 415], KLN [309], C [207, 212, 345], N-UR [150, 207, 212], S-UR [150, 207, 212], SE [207], CAUC [55, 212, 231, 248], GEO [117], AZE [277], KAZ [296], RUS-AS: S-SIB [69, 70, 212], E-SIB [212, 275], YAK [239], ARC-FE [40], N-FE $[94,140]$, S-FE [76, 77, 109, 212]

- sauteri (Bruch et al.) Loeske (P. longifolium var. sauteri (Bruch et al.) C.E.O.Jensen) - UKR: C [66], RUS-EUR: NW [407], CAUC [231], GEO [102, 117]

Pelekium Mitt. [Thuidiaceae]

- minutulum (Hedw.) Touw (Cyrto-hypnum minutulum (Hedw.) W.R.Buck \& H.A.Crum, Microthuidium minutulum (Hedw.) Warnst.) - LTV [244], EST [237], BEL [355], UKR: M [66, 148], RUS-EUR: NW [207], NE [207], C [207, 345], S-UR [91, 150, 207], GEO [117]

- pygmaeum (Bruch et al.) Touw (Cyrto-hypnum pygmaeum (Bruch et al.) W.R.Buck \& H.A.Crum) RUS-AS: S-FE [76, 77, 212]

— versicolor (Müll.Hal.) Touw (Thuidium bipinnatulum Mitt., Cyrto-hypnum sparsifolium (Mitt.) W.R.Buck \& H.A.Crum) - RUS-AS: S-FE [76, 212]

Phascum see Tortula, Microbryum

Philonotis Brid. [Bartramiaceae]

— arnellii Husn. (P. capillaris Lindb.) - LAT [3], UKR: C [66], RUS-EUR: ARC [47, 150, 415], NW [251, 407], NE [415], N-UR [150], RUS-AS: YAK [239], ARC-FE [40]

- caespitosa Jur. - LTV [244], LAT [3], EST [237], BEL [355], UKR: C [66], M [66], RUS-EUR: ARC [47, 150], NW [207, 273, 364, 407], NE [207, 212, 415], KLN [309], C [207], N-UR [90, 150], S-UR [152, 207], SE [207], CAUC [55, 212, 231, 248], GEO [117], ARM [297], AZE [277], KAZ [23], TAD [296], KYR [294, 296, 351], RUS-AS: ARC-ESIB [47], S-SIB [212, 336], E-SIB [212, 386], ARC-YAK [47, 239], YAK [212, 239], ARC-FE [40, 212], N-FE [140] 
- calcarea (Bruch et al.) Schimp. - LTV [244], LAT [3], EST [237], UKR: C [66], M [66], RUS-EUR: NW [207, 364, 407], NE [207, 415], KLN [309], C [207], N-UR [150], S-UR [212, 422], GEO [117], ARM [297], AZE [277], KAZ [156, 157, 296, 356], TUR [296], TAD [294, 296], KYR [267, 296], UZB [267], RUS-AS: S-SIB [197, 212]

— falcata (Hook.) Mitt. - KYR [253], RUS-AS: S-SIB [426]- $\{135\}$

- fontana (Hedw.) Brid. - LTV [244], LAT [3], EST [237], BEL [355], UKR: C [66], M [66], RUS-EUR: ARC [47, 127, 150], NW [207, 212, 364, 407], NE [207, 212, 415], KLN [309], C [207, 212], N-UR [150, 207, 212], S-UR [150, 207, 212], CAUC [55, 212, 231, 248], GEO [117], ARM [297], AZE [277], KAZ [156, 296, 356], TAD [26, 296], KYR [296], UZB [267], RUSAS: ARC-WSIB [136], ARC-ESIB [164, 212, 275], W-SIB [265], S-SIB [69, 70, 212], E-SIB [69, 212, 275], ARC-YAK [47, 212, 239], YAK [212, 239], ARC-FE [40, 212], N-FE [94, 140], S-FE $[76,77,109,212]$

- marchica (Hedw.) Brid. - LTV [244], LAT [3], BEL [355], UKR: C [66], M [66], RUS-EUR: NE [415], KLN [309], C [207, 212], CAUC [231], GEO [117], ARM [297], AZE [277], KAZ [296, 356, 393], UZB [347], TAD [294, 296], KYR [296, 349, 351], RUS-AS: S-FE [268]

- mollis (Dozy \& Molk.) Mitt. - excluded - $\{135\}$

- rigida Brid. - GEO [14, 117]

— seriata Mitt. - LAT [3], UKR: C [66], RUS-EUR: ARC [47, 150], NW [212, 273, 364, 407], NE [415], N-UR [150, 207], S-UR [150, 207, 212], CAUC [55, 212, 231, 248], GEO [117], ARM [297], AZE [277], KAZ [157, 296, 356], UZB [347], TAD [294, 296], KYR [296], RUS-AS: S-SIB [70, 212], E-SIB $[212,275]-\{136\}$

- tomentella Molendo ( $P$. fontana var. pumila (Turner) Brid.) - LAT [3], UKR: C [66], RUS-EUR: ARC [47, 127, 150], NW [212, 273, 364, 407], NE [212, 415], N-UR [150], S-UR [150], CAUC [55, 212, 231, 248], GEO [117], ARM [297], KAZ [157], UZB [347], TAD [294, 296], KYR [296, 351], RUS-AS: ARC-WSIB [136], ARC-ESIB [164, 212], W-SIB [147, 212], S-SIB [69, 70, 212], E-SIB $[69,212]$, ARC-YAK [47, 212, 239], YAK [212, 239], ARC-FE [40, 212], N-FE [94, 140]

- yezoana Besch. \& Cardot - RUS-AS: N-FE [129, 140], S-FE [67, 114] - $\{136\}$

Physcomitrella Bruch et al. [Funariaceae]

- patens (Hedw.) Bruch et al. (Aphanorrhegma patens (Hedw.) Lindb., Physcomitrium patens (Hedw.) Mitt.) - LTV [244], LAT [3], EST [237], BEL [355], UKR: C [66], M [66], MLD [367], RUS-EUR: NW [207, 262], NE [207], C [207, 212, 345], S-UR [91, 150, 207, 212], SE [207], CAUC [232], RUSAS: W-SIB [265], S-SIB [197], E-SIB [275], YAK [239]

Physcomitrium (Brid.) Brid. [Funariaceae]

— arenicola Laz. - UKR: M [66], RUS-EUR: C [207, 345], SE [207], CAUC [226]

- eurystomum Sendtn. - LTV [244], EST [237], BEL [355], UKR: M [66], RUS-EUR: NW [262], ?SUR [150], AZE [277], KAZ [156, 157], UZB [347], KYR [296, 351], RUS-AS: W-SIB [265], S-SIB [69], E-SIB [275], S-FE [76, 212]

- eurystomum subsp. acuminatum (Bruch et al.) Giacom. (P. acuminatum Bruch et al.) - UKR: C [66], M [66], RUS-EUR: CAUC [18, 55], GEO [117], AZE [277], RUS-AS: S-FE [76]

- pyriforme (Hedw.) Hampe - LTV [244], LAT [3], EST [237], BEL [355], UKR: C [66], M [66], MLD [367], RUS-EUR: NW [207, 283], KLN [309], C [207, 212], S-UR [150, 207], SE [207], CAUC [231], GEO [117], ARM [297], AZE [277], UZB [347], TAD [296], KYR [296], RUS-AS: S-SIB $[69,70]$

— sphaericum (C.F.Ludw. ex Schkuhr) Brid. - BEL [355], UKR: M [66], RUS-EUR: NW [407, 262], C [207, 212], GEO [117], AZE [277], KYR [296], RUS-AS: W-SIB [265], S-SIB [70], S-FE [275]

Plagiobryum Lindb. [Bryaceae], see also Bryum - $\{23\}$

- demissum (Hook.) Lindb. (Bryum demissum Hook.) - RUS-EUR: NW [364], CAUC [55], GEO [13, 117], KAZ [157], RUS-AS: ARC-ESIB [212], S-SIB [69, 70, 197], ARC-YAK [47, 239], ARC-FE [40, 212], N-FE [40]

- hultenii (Ochi \& Perss.) Hedd. (P. demissum subsp. hultenii Ochi \& Perss.)- RUS-AS: S-FE [77]

- japonicum Nog. - RUS-AS: S-FE [212, 221]

— zierii (Hedw.) Lindb. (Bryum zieri Hedw.) - UKR: C [66], RUS-EUR: ARC [47], NW [364, 407], 
CAUC [55, 212, 231], GEO [117], KAZ [156, 157],TAD [296], KYR [296], RUS-AS: S-SIB [69, 70, 212]

Plagiomnium T.J.Kop. [Mniaceae]

— acutum (Lindb.) T.J.Kop. - RUS-AS: S-SIB [69, 70], YAK [239], N-FE [140], S-FE [76, 77, 109, 212]

- affine (Blandow ex Funck) T.J.Kop. - LTV [244], LAT [3, 252], EST [237, 252], BEL [355], UKR: C [66, 252], M [66, 252], K [66, 252, 328], MLD [252, 367], RUS-EUR: ARC [47, 150, 415], NW [207, 252, 407], NE [207, 212, 415], KLN [309], C [207, 212, 252], N-UR [150, 207, 415], S-UR [150, 207, 212, 252], SE [207], CAUC [55, 208, 212, 231, 248, 252], GEO [117, 252], ARM [297], AZE [252, 277], KAZ [156, 159, 296, 356], KYR [296] - \{137\}

- confertidens (Lindb. \& Arnell) T.J.Kop. - RUS-EUR: ARC [150], NW [47], NE [212, 415], C [211], N-UR [90, 150, 207, 212], S-UR [150, 207, 212], KAZ [296, 356], RUS-AS: W-SIB [212, 265], SSIB [69, 70, 212], E-SIB [69, 212], YAK [239], S-FE [76, 114, 212, 221]

- curvatulum (Lindb.) Schljakov (P. medium subsp. curvatulum (Lindb.) T.J.Kop.) - RUS-EUR: ARC [47, 127, 150], NW [85, 212, 291, 364], NE [366a], N-UR [150, 212, 417], RUS-AS: ARC-WSIB [136], ARC-ESIB [164, 212], W-SIB [265], S-SIB [252], E-SIB [126, 212], ARC-YAK [47, 212, 239], YAK [239, 252], ARC-FE [40, 212], N-FE [94, 140], S-FE [252]

- cuspidatum (Hedw.) T.J.Kop. - LTV [244], LAT [3], EST [237], BEL [355], UKR: C [66], M [66], K [66, 328], MLD [367], RUS-EUR: ARC [47, 150, 415], NW [207, 212, 364, 407], NE [207, 212, 415], KLN [309], C [207, 212], N-UR [150, 207, 212], S-UR [150, 207, 212], SE [207, 212], CAUC [55, 212, 231, 248], GEO [117], ARM [297], AZE [277], KAZ [156, 159, 296, 356], KYR [296], RUS-AS: ARC-WSIB [?47]; ARC-ESIB [?47]; W-SIB [212, 265], S-SIB [69, 70, 212], E-SIB [69, 212], ARC-YAK [47], YAK [239], ARC-FE [40], N-FE [94, 140], S-FE [76, $77,109,212]$

- drummondii (Bruch \& Schimp.) T.J.Kop. - LAT [3], RUS-EUR: NW [207, 212, 407], NE [207, 212, 415], C [207, 212], N-UR [150, 207], S-UR [150, 207], RUS-AS: W-SIB [265], S-SIB [69, 70, 212], E-SIB [212, 275], YAK [239], N-FE [140], S-FE [76, 114, 212, 221]

- elatum (Bruch et al.) T.J.Kop. - LTV [244], LAT [3, 252], EST [237, 252], BEL [252, 355], UKR: C [66], M [66], K [328], RUS-EUR: ARC [47, 150], NW [207, 212, 252, 364, 407], NE [207, 212, 252, 415], KLN [309], C [207, 212, 252], N-UR [150], S-UR [150, 207, 212], SE [207], CAUC [55, 212, 231], GEO [117], ARM [297], AZE [277], KAZ [296] - \{137\}

- ellipticum (Brid.) T.J.Kop. - LTV [244], LAT [3], EST [237], BEL [355], UKR: C [66], M [66], K [328], MLD [367], RUS-EUR: ARC [47, 127, 150], NW [207, 212, 364, 407], NE [207, 212, 415], KLN [309], C [207, 212], N-UR [150, 207, 212], S-UR [150, 207, 212], SE [207, 212], CAUC [55, 212, 231, 248], GEO [117], ARM [297], AZE [277], KAZ [159, 296, 356], TAD [294, 296], KYR [296, 351], RUS-AS: ARC-WSIB [136], ARC-ESIB [164, 212], W-SIB [212, 265], S-SIB [69, 70, 212], E-SIB [69, 212], ARC-YAK [47, 212], YAK [212, 239], ARC-FE [40, 212], N-FE [94, 140], SFE $[76,77,109,212]$

- japonicum (Lindb.) T.J.Kop. - RUS-AS: S-FE [37, 75, 212]

- maximowiczii (Lindb.) T.J.Kop. - RUS-AS: E-SIB [212, 247], S-FE [76, 212, 221]

- medium (Bruch et al.) T.J.Kop. - LTV [244], LAT [3], EST [237], BEL [355], UKR: C [66], M [66], K [66, 328], RUS-EUR: ARC [47, 150], NW [207, 212, 364, 407], NE [207, 212, 415], KLN [309], C [207, 212], N-UR [150, 207, 212], S-UR [150, 207, 212], SE [207, 212], CAUC [55, 212, 231, 248], GEO [117], ARM [297], AZE [277], KAZ [156, 159, 160, 296], UZB [347], TAD [296], KYR [296, 351], RUS-AS: ARC-ESIB [212], W-SIB [265], S-SIB [69, 70, 212], E-SIB [69, 212], ARC-YAK [47, 239], YAK [239], ARC-FE [40], N-FE [94, 140], S-FE [76, 77, 109, 212]

— rostratum (Schrad.) T.J.Kop. - LTV [244], LAT [3], EST [237], BEL [355], UKR: C [66], M [66], K [66, 328], MLD [367], RUS-EUR: ARC [47, 150], NW [207, 407], NE [207, 212, 415], KLN [309], C [207, 212], N-UR [150, 207, 212], S-UR [150, 207, 212], CAUC [55, 208, 212, 231, 248], GEO [117], ARM [297], AZE [277], KAZ [156, 159, 160], TAD [296], KYR [296, 351], RUS-AS: ARCWSIB [136], W-SIB [265], S-SIB [69, 70, 212], E-SIB [212, 247], ARC-YAK [47, 239], YAK [239], N-FE [140], S-FE [76, 109, 212] $-\{138\}$

— undulatum (Hedw.) T.J.Kop. - LTV [244], LAT [3], EST [237], BEL [355], UKR: C [66], M [66], K 
[66, 328], MLD [367], RUS-EUR: NW [207], KLN [309], C [207, 212], CAUC [55, 208, 212, 231, 248], GEO [117], ARM [297], AZE [277] - \{139\}

— vesicatum (Besch.) T.J.Kop. - RUS-AS: E-SIB [212, 247], S-FE [76, 77, 212]

Plagiopus Brid. [Bartramiaceae]

- oederianus (Sw.) H.A.Crum \& L.E.Anderson (P. oederi (Brid.) Limpr.) - LAT [3], EST [237], UKR: C [66], M [66], K [66, 328], RUS-EUR: ARC [47, 150], NW [212, 364, 407], NE [207, 212, 415], C [207], N-UR [150, 207, 212], S-UR [150, 207, 212], CAUC [55, 212, 231, 248], GEO [117], ARM [297], AZE [277], KAZ [156], TAD [296], KYR [296, 349, 351], RUS-AS: ARC-ESIB [164], W-SIB [338], S-SIB [69, 70, 212], E-SIB [69, 212, 275], ARC-YAK [47, 239], YAK [212, 239], ARC-FE [40, 212], N-FE [140], S-FE [76, 77, 212]

Plagiothecium Bruch et al. [Plagiotheciaceae]

- berggrenianum Frisvoll - RUS-EUR: ARC [47, 42], RUS-AS: ARC-WSIB [136], ARC-ESIB [39, 164, 212], E-SIB [163], ARC-YAK [47, 212, 239], YAK [239], ARC-FE [40, 212], N-FE [40] $\{140\}$

- cavifolium (Brid.) Z.Iwats. (P. roeseanum Bruch et al.) - LTV [244], LAT [3], EST [237], BEL [355], UKR: C [66], M [66], MLD [367], RUS-EUR: ARC [47, 150], NW [207, 212, 364, 407], NE [207, 212, 415], KLN [309], C [207, 212], N-UR [150, 207, 212], S-UR [150, 207, 212], SE [207, 212], CAUC [55, 212, 231, 248], GEO [117], AZE [277], KAZ [296], RUS-AS: ARC-WSIB [136], ARCESIB [164, 212], W-SIB [147, 212], S-SIB [69, 70, 212], E-SIB [69, 212], ARC-YAK [47, 212, 239], YAK [239], ARC-FE [40, 212], N-FE [94, 140], S-FE [76, 77, 109, 212]

— ?cordifolium Laz. - RUS-AS: S-FE $[268,76]-\{141\}$

- curvifolium Schlieph. ex Limpr. (P. laetum var. curvifolium (Limpr.) Mastracci \& M.Sauer) - LTV [244], LAT [3], EST [237], UKR: C [66], M [66], K [328], RUS-EUR: NW [207, 364, 407], NE [212, 415], C [207, 212, 345], N-UR [415], S-UR [91], RUS-AS: S-FE [76, 77, 212] - $\{142\}$

- denticulatum (Hedw.) Bruch et al. - LTV [244], LAT [3], EST [237], BEL [355], UKR: C [66], M [66], RUS-EUR: ARC [47, 127, 150], NW [207, 212, 364, 407], NE [207, 212, 415], KLN [309], C [207, 212], N-UR [150, 207, 212], S-UR [150, 207, 212], SE [207, 212], CAUC [55, 212, 231, 248], GEO [117], ARM [297], AZE [277], KAZ [159, 296, 356], TAD [296], KYR [296, 351], RUSAS: ARC-WSIB [136], ARC-ESIB [164, 212], W-SIB [212, 265], S-SIB [69, 70, 212], E-SIB [69, 212, 275], ARC-YAK [47, 239], YAK [239], ARC-FE [40, 212], N-FE [94, 140], S-FE [76, 109, 212]

— denticulatum var. obtusifolium (Turner) Moore - UKR [66], RUS-EUR: NW [364]

- denticulatum var. undulatum R.Ruthe ex Geh. (P. denticulatum var. ruthei (Limpr.) Riehm., P. ruthei Limpr.) - LTV [244], LAT [3], EST [237], UKR [66], RUS-EUR: NE [415], C [345], KLN [309], C [207, 212]

- euryphyllum (Cardot \& Thér.) Z.Iwats. - RUS-AS: N-FE [140], S-FE [114]

— laetum Bruch et al. - LTV [244], LAT [3], EST [237], BEL [355], UKR: C [66], M [66], RUS-EUR: ARC [47, 127, 150], NW [207, 212, 364, 407], NE [207, 212, 415], KLN [309], C [207, 212], N-UR [150, 207, 212], S-UR [150, 207, 212], SE [207, 212], CAUC [55, 212, 231, 248], GEO [117], AZE [277], KAZ [156, 159, 296, 356], KYR [296, 351], RUS-AS: ARC-WSIB [136], ARC-ESIB [164, 212], W-SIB [212, 265], S-SIB [69, 70, 212], E-SIB [69, 212, 275], ARC-YAK [47, 239], YAK [239], ARC-FE [40, 212], N-FE [94, 140], S-FE [76, 109, 212]

- latebricola Bruch et al. - LTV [244], LAT [3], EST [237], BEL [355], UKR: C [66], M [66, 405], RUS-EUR: NW [207, 212, 407], NE [90, 207, 212], KLN [309], C [207, 212, 345], N-UR [150, 207, 212], S-UR [150, 207, 212], SE [207], GEO [117], KYR [296], RUS-AS: W-SIB [265], S-SIB [200, 212], N-FE [140], S-FE [76, 77, 109, 212]

- neckeroideum Bruch et al. - UKR: C [66], RUS-AS: S-SIB [70] - $\{143\}$

- nemorale (Mitt.) A.Jaeger - LTV [244], LAT [3], EST [237], BEL [355], UKR: C [66], M [66], K [328], RUS-EUR: ?ARC [150], NW [212, 291, 292], NE [212, 415], KLN [309], C [207, 212, 345], N-UR [150], S-UR [150], SE [207], CAUC [55, 208, 212, 231, 248], GEO [117], ARM [297], AZE [277], RUS-AS: N-FE [140], S-FE [76, 77, 109, 212]

- obtusissimum Broth. - RUS-AS: S-FE [76, 77, 109, 212] 
— piliferum (Sw.) Bruch et al. - UKR: C [66], RUS-EUR: ARC [150], NW [207, 212, 364, 407], N-UR [150, 415], RUS-AS: S-SIB [69], E-SIB [69], YAK [389], S-FE [76, 109] $-\{144\}$

- ?platycladum (Cardot) Broth. - RUS-AS: S-FE [268] - $\{141\}$

— platyphyllum Mönk. - UKR: M [400], GEO [117], RUS-EUR: NW [407], ?RUS-AS: N-FE [140]

- ?succulentum (Wilson) Lindb. - LTV [244], LAT [3], EST [237], UKR: C [66], M [66], RUS-EUR: NW [62, 364], NE [212, 415], C [207, 212], N-UR [212, 415]; S-UR [207, 212], GEO [117], RUSAS: S-SIB [69, 70], E-SIB [212, 247], S-FE [76, 212] - \{145\}

- svalbardense Frisvoll - RUS-AS: ARC-FE [40]

- undulatum (Hedw.) Bruch et al. - LTV [244], LAT [3], EST [237], BEL [355], UKR: C [66], RUSEUR: KLN [309], AZE [277], RUS-AS: ARC-FE [40]

Plasteurhynchium M.Fleisch. ex Broth. [Brachytheciaceae]

- meridionale (Bruch et al.) M.Fleisch. (Eurhynchium meridionale (Bruch et al.) De Not.) - UKR: M, K [328], GEO [117]

- striatulum (Spruce) M.Fleisch. (Eurhynchium striatulum (Spruce) Bruch et al., Isothecium filescens (Brid.) Mönk.) - UKR: C [66], M [402], K [66, 328], RUS-EUR: NW [407], CAUC [208, 212, 248]

Platydictya Berk. [Plagiotheciaceae], see also Bardunovia, Serpoleskea subtilis, S. confervoides

- jungermannioides (Brid.) H.A.Crum (Amblystegium jungermannioides (Brid.) A.J.E.Sm.) - LAT [3], EST [237], UKR: C [66], RUS-EUR: ARC [47, 127, 150], NW [212, 364, 407], NE [207, 212, 415], C [207], N-UR [150, 207, 212], S-UR [150, 212], SE [207, 212], CAUC [55, 102, 212, 231], GEO [117], KAZ [393], TAD [296], KYR [296, 351], RUS-AS: ARC-WSIB [136], ARC-ESIB [164, 212], W-SIB [265], S-SIB [69, 70, 212, 275], E-SIB [74, 212], ARC-YAK [47, 239], YAK [212, 239], ARCFE [40, 212], N-FE [140], S-FE [76, 77, 212]

Platygyrium Bruch et al. [Pylaisiadelphaceae]

- repens (Brid.) Bruch et al. - LTV [244], LAT [3], EST [237], BEL [355], UKR: C [66], M [66], MLD [367], RUS-EUR: ARC [47, 150, 415], NW [207, 212, 407], NE [207], KLN [309], C [207, 212], N-UR [150, 207], S-UR [150, 207, 212], SE [207, 212], CAUC [208, 212, 248], GEO [117], ARM [297], AZE [277], KAZ [156, 296, 356], RUS-AS: W-SIB [265], S-SIB [69, 70, 212], E-SIB [74, 212], YAK [212, 239], N-FE [140], S-FE [76, 109, 212]

Platyhypnidium see Rhynchostegium

Pleuridium Rabenh. [Ditrichaceae], see also Cleistocarpidium palustre

— acuminatum Lindb. - BEL [355], UKR: C [66], M [66], K [66, 328] - \{146\}

- subulatum (Hedw.) Rabenh. (P. alternifolium auct. non (Dicks. ex Hedw.) Rabenh.) - LTV [244], LAT [3], EST [237], BEL [355], UKR: C [66], M [66]), MLD [367], RUS-EUR: NW [207, 407], NE [207], KLN [309], C [207, 212, 345], S-UR [150, 207], SE [207], CAUC [232], GEO [117], AZE [277], RUS-AS: W-SIB [339], N-FE [140], S-FE [268]

Pleurochaete Lindb. [Pottiaceae]

- squarrosa (Brid.) Lindb. - UKR: K [66, 328], RUS-EUR: CAUC [232, 6], GEO [117], ARM [297], AZE [277], TAD [295], TUR [267], KYR [296]

Pleuroweisia see Molendoa

Pleuroziopsis Kindb. ex E.Britton [Climaciaceae]

— ruthenica (Weinm.) Kindb. ex E.Britton - RUS-AS: N-FE [140], S-FE [76, 77, 109, 212]

Pleurozium Mitt. [Hylocomiaceae]

- schreberi (Brid.) Mitt. - LTV [244], LAT [3], EST [237], BEL [355], UKR: C [66], M [66], K [66, 328], MLD [367], RUS-EUR: ARC [47, 127, 150], NW [207, 212, 364, 407], NE [207, 212, 415], KLN [309], C [207, 212], N-UR [150, 207, 212], S-UR [150, 207, 212], SE [207, 212], CAUC [55, 212, 231, 248], GEO [117], ARM [297], AZE [277], KAZ [159, 296, 356], TAD [26, 296], KYR [296], RUS-AS: ARC-WSIB [136], ARC-ESIB [212], W-SIB [212, 265], S-SIB [69, 70, 212], E-SIB [69, 212, 275], ARC-YAK [47, 212, 239], YAK [212, 239], ARC-FE [40], N-FE [94, 140], S-FE [76, $77,109,212]$

Podperaea Z.Iwats. \& Glime [*Amblystegiaceae]

- krylovii (Podp.) Z.Iwats. \& Glime - RUS-AS: S-SIB [69, 197, 212], S-FE [76, 110, 212, 221]

Pogonatum P.Beauv. [Polytrichaceae], see also Polytrichastrum alpinum 
- aloides (Hedw.) P.Beauv. - LTV [244], LAT [3], EST [237], BEL [355], UKR: C [66], M [66], K [328], RUS-EUR: KLN [309], CAUC [55, 212, 231, 248], GEO [117], AZE [277]

- contortum (Brid.) Lesq. (P. laterale (Brid). Brid.) - RUS-AS: N-FE [140], S-FE [76, 77, 109, 212]

- dentatum (Brid.) Brid. (P. capillare (Michx.) Brid.) - LAT [3], EST [237], RUS-EUR: ARC [47, 127, 150], NW [207, 212, 364, 407], NE [207, 212, 415], C [207], N-UR [150, 207, 212], S-UR [150, 207], RUS-AS: ARC-WSIB [136], ARC-ESIB [164, 212], W-SIB [265], S-SIB [69, 212, 219], E-SIB [69, 212], ARC-YAK [47, 212, 239], YAK [212, 239], ARC-FE [40, 212], N-FE [94, 140], S-FE [76, 77, $109,212]-\{147\}$

— inflexum (Lindb.) Sande Lac. - RUS-AS: S-FE [76, 77] - $\{148\}$

- japonicum Sull. \& Lesq. - RUS-AS: N-FE [140], S-FE [76, 77, 109, 212]

- nanum (Hedw.) P.Beauv. - LTV [244], LAT [3], EST [237], BEL [355], UKR: C [66, 256], M [66], K [66, 328], RUS-EUR: NW [207, 262], KLN [309], C [207], GEO [117]

— neesii (Müll.Hal.) Dozy - RUS-EUR: CAUC [55, 56], GEO [56]

- spinulosum Mitt. - RUS-AS: S-FE [76, 77, 212]

- urnigerum (Hedw.) P.Beauv. - LTV [244], LAT [3], EST [237], BEL [355], UKR: C [66], M [66], K [66, 328], MLD [367], RUS-EUR: ARC [47, 127, 150], NW [207, 212, 364, 407], NE [207, 212, 415], KLN [309], C [207, 212], N-UR [150, 207, 212], S-UR [150, 207, 212], SE [207], CAUC [55, 212, 231, 248], GEO [117], ARM [297], AZE [277], KAZ [296, 356], RUSAS: ARC-WSIB [136], ARC-ESIB [164, 212], W-SIB [212, 265], S-SIB [69, 70, 212, 219], ESIB [69, 212], ARC-YAK [47, 212, 239], YAK [212, 239], ARC-FE [40, 212], N-FE [94, 140], SFE [76, 77, 109, 212]

Pohlia Hedw. [Mielichhoferiaceae]

— alba Lindb. \& Arnell - RUS-AS: W-SIB [?265], E-SIB [275]

— andalusica (Höhn.) Broth. - LAT [132], UKR: M [66, 132], RUS-EUR: NW [100, 132, 207, 212 , 291], NE [132, 207, 212], C [132, 207, 212], N-UR [90, 150, 207, 212], S-UR [150, 207, 212], CAUC [231, 248], RUS-AS: ARC-WSIB [?47], W-SIB [132, 212], S-SIB [132], ARC-FE [40, 132], N-FE $[94,132,140]$

- andrewsii A.J.Shaw - RUS-EUR: ARC [47, 127, 150], NW [132], NE [132, 207, 212, 416], N-UR [90, 150, 207, 212], S-UR [132, 207, 212, 150], CAUC [212, 248], RUS-AS: ARC-WSIB [132, 136], ARC-ESIB [132, 164, 212,], S-SIB [132, 212], E-SIB [132], ARC-YAK [47, 132, 239], YAK [132, 239], ARC-FE [40, 212, 132], N-FE [132, 140], S-FE [212, 221]

- annotina (Hedw.) Lindb. - LTV [244], BEL [355], UKR: M [66], RUS-EUR: ARC [150], NW [207, 273, 364], NE [132, 207, 212, 415], KLN [309], C [132, 207, 212], N-UR [150], S-UR [150, 207, 212], ARM [297], RUS-AS: S-SIB [132, 212], E-SIB [212, 247], ARC-YAK [239], YAK [239], N-FE $[94,132,140]$

- atropurpurea (Wahlenb.) H.Lindb. - BEL [355], RUS-EUR: ARC [47], NW [207, 364], NE [207], RUS-AS: ARC-WSIB [136], S-SIB [197, 212], E-SIB [126, 212]

- beringiensis A.J.Shaw - RUS-EUR: ARC [42], RUS-AS: ARC-WSIB [136], ARC-ESIB [39, 47, 132], S-SIB [132], E-SIB [132], ARC-FE [40, 132, 212], N-FE [40, 132]

- brevinervis Lindb. \& Arnell - RUS-AS: E-SIB [275]

- bulbifera (Warnst.) Warnst. - LTV [244], LAT [3], EST [237], BEL [355], UKR: M [66], RUSEUR: ARC [47, 127, 150], NW [132, 207, 212, 364, 407], NE [132, 207, 212], C [132, 207, 212], NUR [90, 207], S-UR [150, 207, 212], RUS-AS: ARC-WSIB [132, 136], W-SIB [132, 212, 265], S-SIB [132, 212], E-SIB [132, 212], ARC-YAK [47, 212, 239], YAK [132, 212, 239], ARC-FE [40, 132, 212], N-FE [132, 140], S-FE [132]

- camptotrachela (Renauld \& Cardot) Broth. - LTV [244], LAT [3], EST [237], UKR: C [401], M [132], RUS-EUR: NW [132, 207, 212, 407], C [132, 207, 212]

- cardotii (Renauld \& Cardot) Broth. - RUS-AS: N-FE [130, 140]

- cruda (Hedw.) Lindb. - LTV [244], LAT [3], EST [237], BEL [355], UKR: C [66], M [66], K [66, 328], RUS-EUR: ARC [47, 127, 150], NW [207, 212, 364, 407], NE [207, 212, 415], KLN [309], C [207, 212], N-UR [150, 207, 212], S-UR [150, 207, 212], SE [207, 212], CAUC [55, 212, 231, 248], 
GEO [117], ARM [297], AZE [277], KAZ [156, 159, 296, 356], TUR [296], TAD [294, 296], KYR [296, 351], UZB [267], RUS-AS: ARC-WSIB [136], ARC-ESIB [164, 212, 275], W-SIB [212, 265], S-SIB [69, 70, 212], E-SIB [69, 212, 275], ARC-YAK [47, 212, 239], YAK [212, 239], ARC-FE [40, 212], N-FE [94, 140], S-FE [76, 77, 114, 212]

- crudoides (Sull. \& Lesq.) Broth. - RUS-EUR: ARC [47, 127, 150, 415], NW [87, 364], N-UR [207], S-UR [150, 207, 212], RUS-AS: ARC-WSIB [136], ARC-ESIB [164, 212], S-SIB [69, 70, 212], ESIB [69, 212], ARC-YAK [47, 212, 239], YAK [212, 239], ARC-FE [40, 212], N-FE [94, 140], S-FE $[212,221]$

- drummondii (Müll.Hal.) A.L.Andrews - UKR: C [66], RUS-EUR: ARC [47, 127, 132, 150], NW [132, 212, 273, 364, 283], NE [366a, 417a], N-UR [150, 207, 212, 415], CAUC [132, 231], GEO [117], RUS-AS: ARC-WSIB [132, 136], ARC-ESIB [132, 164, 212,], W-SIB [132, 212], S-SIB [69, 70, 132, 212], E-SIB [126, 212], ARC-YAK [47, 132, 239], YAK [212, 239], ARC-FE [40, 132, 212], N-FE [94, 132, 140], S-FE [132, 212, 221]

- elongata Hedw. - LAT [3], EST [237], UKR: C [66], M [66], RUS-EUR: ARC [47, 150], C [207, 345], N-UR [150, 207, 212, 415], S-UR [150, 207, 212], CAUC [55, 212, 231, 248], GEO [117], ARM [297], KAZ [296, 356], TAD [296], KYR [296], RUS-AS: ARC-ESIB [?47]; S-SIB [69, 70, 212], E-SIB [74, 212, 247], ARC-YAK [239], YAK [239], ARC-FE [40], S-FE [76, 212, 221]

- elongata var. greenii (Brid.) A.J.Shaw (P. ambigua (Limpr.) Broth., P. minor auct.) - UKR: C [66], RUS-EUR: ARC [47, 150], NW [364], NE [414], N-UR [150, 414], CAUC [212, 231, 248], GEO [117], ARM [297], AZE [277], KAZ [159, 296, 356], TAD [296], KYR [296], RUS-AS: ARC-WSIB [136], S-SIB [69, 70, 212], E-SIB [69], ARC-FE [40], S-FE [76, 212, 221]

- filum (Schimp.) Mårtensson - LTV [244], LAT [3], BEL [355], UKR: C [66], RUS-EUR: ARC [47, 127, 132, 150, 364], NW [132, 207, 273, 286, 364], NE [207, 212, 415], ?C [132], N-UR [150, 212, 415], CAUC [132, 212, 231, 248], GEO [117, ARM [297], ?TAD [296], RUS-AS: ARC-WSIB [132, 136], ARC-ESIB [132], W-SIB [132, 212], S-SIB [70, 212], E-SIB [132, 212], ARC-YAK [47, 239], YAK [132, 239], ARC-FE [40, 132, 212], N-FE [94, 132, 140]

- lescuriana (Sull.) Ochi (P. pulchella (Hedw.) Lindb.) - LTV [244], LAT [3], EST [237], UKR: C [66], M [66], RUS-EUR: ARC [47, 127, 150, 415], NW [207, 212, 364, 407], NE [415], KLN [309], C [207, 212], N-UR [90, 150, 207, 415], RUS-AS: ARC-ESIB [275], W-SIB [265], E-SIB [275], ARC-YAK [47, ?239], YAK [?239], ARC-FE [40], N-FE [40]

- leucostoma (Bosch \& Sande Lac.) M.Fleisch. (P. gracillima (Cardot) Horik. \& Ochi) -?RUS-AS: ESIB $[212,247]-\{149\}$

- longicollis (Hedw.) Lindb. - UKR: C [66], RUS-EUR: ARC [47, 150], NW [364], NE [212, 415], NUR [150, 207, 212], S-UR [150, 207, 212], CAUC [55, 212, 231, 248], GEO [117], AZE [277], KAZ [156, 159, 356], KYR [296], RUS-AS: S-SIB [69, 70, 212], E-SIB [212, 247], ARC-YAK [47, 239], YAK [239], ARC-FE [40], N-FE [94, 140], S-FE [76, 212, 221]

- ludwigii (Spreng. ex Schwägr.) Broth. - UKR: C [66, 413], RUS-EUR: ARC [47], NW [87, 364], NUR [150, 207, 415], S-UR [150], CAUC [212, 231], GEO [117]

- melanodon (Brid.) A.J.Shaw (Mniobryum delicatulum (Hedw.) Dixon) - LTV [244], LAT [3], EST [237], BEL [355], UKR: C [66], M [66], K [66, 328], MLD [367], RUS-EUR: NW [207], NE [207, 415], KLN [309], C [207, 212], N-UR [207], S-UR [150, 207], SE [207], GEO [117], ARM [297], AZE [277], TUR [296], UZB [347], TAD [296], KYR [296], RUS-AS: W-SIB [265], S-SIB [70, 212], S-FE [77, 109]

- nutans (Hedw.) Lindb. - LTV [244], LAT [3], EST [237], BEL [355], UKR: C [66], M [66], K [66, 328], RUS-EUR: ARC [47, 127, 150], NW [207, 212, 364, 407], NE [207, 212, 415], KLN [309], C [207, 212], N-UR [150, 207, 212], S-UR [150, 207, 212], SE [207, 212], CAUC [55, 212, 231, 248], GEO [117], ARM [297], AZE [277], KAZ [156, 159, 296], TAD [294, 296], KYR [296, 351], RUS-AS: ARC-WSIB [136], ARC-ESIB [164, 212], W-SIB [212, 265], S-SIB [69, 70, 212], E-SIB $[69,212]$, ARC-YAK [47, 212, 239], YAK [212, 239], ARC-FE [40, 212], N-FE [94, 140], S-FE $[76,109,212]$

- nutans subsp. schimperi (Müll.Hal.) Nyholm - RUS-EUR: ARC [47, 127], NW [364], RUS-AS: ARC-ESIB [50], ARC-FE [40] 
- obtusifolia (Vill. ex Brid.) L.F.Koch - UKR: C [66], RUS-EUR: ARC [47, 150], NW [87, 212, 364, 407], NE [415], N-UR [150, 415], CAUC [212, 231, 248], GEO [117], RUS-AS: ARC-ESIB [?47], SSIB [70, 212], ARC-YAK [47], ARC-FE [40]

- proligera (Kindb.) Lindb. ex Broth. - LTV [244], LAT [3], EST [237], BEL [355], RUS-EUR: ARC [127, 132, 150], NW [132, 207, 212, 364, 407], NE [132, 207, 212, 415], C [132, 207, 212], N-UR [132, 150, 207, 212], S-UR [132, 150, 207], CAUC [55, 212, 231, 248], GEO [117], RUS-AS: ARCWSIB [132, 136], ARC-ESIB [132, 164, 212], W-SIB [132, 212, 265], S-SIB [132, 212], E-SIB [132, 212], ARC-YAK [132, 212, 239], YAK [132, 239], ARC-FE [40, 132, 212], N-FE [94, 132, 140], S-FE $[114,132,212,221]$

— saprophila (Müll.Hal.) Broth. - RUS-EUR: ?NE [415], KAZ [157, 296, 356], KYR [296]

- sphagnicola (Bruch et al.) Broth. - LTV [244], LAT [3], EST [237], BEL [355], RUS-EUR: ARC [47, 127, 150], NE [415], KLN [309], N-UR [150, 415], S-UR [150], KAZ [296, 356], RUS-AS: ARC-WSIB [136], ARC-ESIB [?47, 275], W-SIB [212, 265], S-SIB [212, 336], E-SIB [126, 212], ARC-FE [40], S-FE [109, 212]

— torrentium (I.Hagen) Broth. - RUS-EUR: NW [364], RUS-AS: ARC-FE [40], N-FE [140] — $\{150\}$

- tundrae A.J.Shaw - RUS-AS: ARC-WSIB [144], N-FE [140, 144]

— vexans (Limpr.) H.Lindb. - RUS-EUR: NE [207], RUS-AS: S-SIB [197, 212], ARC-FE [40]

— viridis Lindb. \& Arnell - RUS-AS: E-SIB [275]

- wahlenbergii (F.Weber \& D.Mohr) A.L.Andrews (Mniobryum wahlenbergii (F.Weber \& D.Mohr) Jenn., M. wahlenbergii var. glacialis (Brid.) Wijk \& Margad., Pohlia wahlenbergii var. glacialis (Brid.) E.F.Warb.) - LTV [244], LAT [3], EST [237], BEL [355], UKR: C [66], M [66], K [66, 328], MLD [367], RUS-EUR: ARC [47, 150], NW [207, 212, 364, 407], NE [207, 212, 415], KLN [309], C [207, 212], N-UR [150, 207, 212], S-UR [150, 207, 212], SE [207], CAUC [55, 208, 212, 231, 248], GEO [117], ARM [297], AZE [277], KAZ [296, 356], TUR [296], TAD [294, 296], KYR [296], UZB [267], RUS-AS: ARC-WSIB [136], ARC-ESIB [212, 263], W-SIB [212, 265], SSIB [69, 70, 212], E-SIB [69, 212], ARC-YAK [47, ?239], YAK [212, 239], ARC-FE [40, 212], NFE [94, 140], S-FE [212, 221]

Polytrichastrum G.L.Sm. [Polytrichaceae]

- alpinum (Hedw.) G.L.Sm. (Polytrichum alpinum Hedw.) - UKR: C [66], RUS-EUR: ARC [47, 127, 150], NW [212, 273, 364, 407], NE [207, 212, 415], N-UR [150, 207, 212], S-UR [150, 207, 212], CAUC [55, 212, 231, 248], GEO [117], ARM [297], AZE [277], KAZ [296, 356], TAD [295], KYR [351], RUS-AS: ARC-WSIB [136], ARC-ESIB [164, 212], W-SIB [147, 212], S-SIB [69, 70, 212, 219], E-SIB [69, 212], ARC-YAK [47, 212, 239], YAK [212, 239], ARC-FE [40, 212], N-FE [94, 140], S-FE [76, 77, 109, 212]

- alpinum var. fragile (Bryhn) D.G.Long - RUS-EUR: ARC [47, 150, 414], NW [291, 364], NE [414], KAZ [159, 356], RUS-AS: ARC-WSIB [136], ARC-ESIB [212], E-SIB [126, 212, 386], ARC-YAK [47, 212, 239], YAK [239], ARC-FE [40, 212], N-FE [40, 94]

- alpinum var. septentrionale (Sw.) G.L.Sm. (P. norwegicum (Hedw.) Schljakov) - RUS-EUR: ARC [47, 150], NW [273, 364], NE [414], N-UR [150], S-UR [150], RUS-AS: ARC-WSIB [136], ARC-ESIB [?47], S-SIB [70, 219, 324], ARC-YAK [47], YAK [239], ARC-FE [40], N-FE [94, 140]

— altaicum Ignatov \& G.L.Sm. - RUS-AS: S-SIB [219]

- formosum (Hedw.) G.L.Sm. (Polytrichum formosum Hedw.) - LTV [244], LAT [3], EST [237], BEL [355], UKR: C [66], M [66], K [66, 328], MLD [367], RUS-EUR: NW [207, 212, 364, 407], NE [207, 212], KLN [309], C [207, 212], N-UR [150, 207, 212, 415], S-UR [150, 207, 212], SE [207], CAUC [55, 212, 231, 248], GEO [117], ARM [297], AZE [277], KYR [351], RUS-AS: WSIB [265], S-SIB [70, 212, 219], E-SIB [212, 247], YAK [239], ARC-FE [40], N-FE [140], S-FE $[212,77,109]$

- longisetum (Sw. ex Brid.) G.L.Sm. (Polytrichum longisetum Sw. ex Brid., P. longisetum var. anomalum (Milde) Ignatov \& G.L.Sm.) - LTV [244], LAT [3], EST [237], BEL [355], UKR: C [66], M [66], RUS-EUR: ARC [47, 127, 150], NW [207, 212, 364, 407], NE [207, 212, 415], KLN [309], C [207, 212], N-UR [90, 150, 207, 212], S-UR [150, 207, 212], SE [207], CAUC [55, 212, 231, 248], GEO [117], ARM [297], AZE [277], KAZ [296], RUS-AS: ARC-WSIB [136], ARC-ESIB [?47], W- 
SIB [212, 265], S-SIB [69, 70, 212, 219], E-SIB [74, 212, 247], ARC-YAK [47, 212, 239], YAK [239], ARC-FE [40], N-FE [94, 140], S-FE [76, 77, 109, 212]

- pallidisetum (Funck) G.L.Sm. (Polytrichum formosum var. decipiens (Limpr.) Loeske, Polytrichum pallidisetum Funck, Polytrichum ochioense auct. non Renauld \& Cardot) - LAT [3], EST [237], UKR: C [66], M [66], RUS-EUR: NW [207, 212, 262, 407], NE [207, 212], KLN [309], C [207, 212], N-UR [150, 207, 212, 415], S-UR [150, 207, 212], GEO [117], RUS-AS: S-SIB [70, 212, 219], E-SIB [212, 247], YAK [?239], S-FE [76, 77, 109, 212]

- sexangulare (Flörke ex Brid.) G.L.Sm. (Polytrichum sexangulare Flörke ex Brid.) - UKR: C [66], RUS-EUR: ARC [47, 127, 150], NW [87, 273, 364], N-UR [150, 207, 212], S-UR [150], CAUC [55, 212, 231, 248], GEO [117], ARM [297], RUS-AS: ARC-ESIB [212, 263], S-SIB [69, 70, 212, 219], YAK [239], ARC-FE [40, 212], N-FE [94, 140], S-FE [212, 221]

- sphaerothecium (Besch.) J.-P.Frahm (Polytrichum sphaerothecium (Besch.) Müll.Hal.) - RUS-AS: ARC-FE [43], N-FE [140], S-FE [77, 110, 415]

Polytrichum Hedw. [Polytrichaceae], see also Polytrichastrum

- commune Hedw. - LTV [244], LAT [3], EST [237], BEL [355], UKR: C [66], M [66], RUS-EUR: ARC [47, 127, 150], NW [207, 212, 364, 407], NE [207, 212, 415], KLN [309], C [207, 212], N-UR [150, 207, 212], S-UR [150, 207, 212], SE [207, 212], CAUC [55, 212, 231, 248], GEO [117], ARM [297], AZE [277], KAZ [296, 356], TAD [295], KYR [296], RUS-AS: ARC-WSIB [136], ARCESIB [?47], W-SIB [212, 265], S-SIB [69, 70, 212, 219], E-SIB [69, 212], ARC-YAK [47, 239], YAK $[212,239]$, ARC-FE [40], N-FE [94, 140], S-FE [76, 77, 109, 212] - $\{151\}$

- hyperboreum R.Br. - RUS-EUR: ARC [47, 127, 150], NW [87, 212, 262, 364, 407], NE [415], NUR [150, 212, 415], S-UR [150], RUS-AS: ARC-WSIB [136], ARC-ESIB [164, 212], S-SIB [69, 70, 212], E-SIB [212, 386], ARC-YAK [47, 212, 239], YAK [212, 239], ARC-FE [40, 212], N-FE $[94,140]$

- jensenii I.Hagen - RUS-EUR: ARC [47, 127, 150], NW [212, 262, 364, 407], NE [414], N-UR [150], RUS-AS: ARC-WSIB [136], ARC-ESIB [164, 212], W-SIB [212, 265], S-SIB [69, 70, 212], E-SIB [69, 212], ARC-YAK [47, 212, 239], YAK [212, 239], ARC-FE [40, 212], N-FE [94, 140], SFE [77, 212]

- juniperinum Hedw. - LTV [244], LAT [3], EST [237], BEL [355], UKR: C [66], M [66], K [66, 328], MLD [367], RUS-EUR: ARC [47, 127, 150], NW [207, 212, 364, 407], NE [207, 212, 415], KLN [309], C [207, 212], N-UR [150, 207, 212], S-UR [150, 207, 212], SE [207], CAUC [55, 212, 231, 248], GEO [117], ARM [297], AZE [277], KAZ [156, 296, 356], UZB [267], TAD [295], KYR [296, 349, 351], RUS-AS: ARC-WSIB [136], ARC-ESIB [164, 212], W-SIB [212, 265], S-SIB [69, 70, 212, 219], E-SIB [69, 212], ARC-YAK [47, 212, 239], YAK [212, 239], ARC-FE [40, 212], N-FE $[94,140]$, S-FE [76, 77, 109, 212]

— piliferum Hedw. - LTV [244], LAT [3], EST [237], BEL [355], UKR: C [66], M [66], K [66, 328], MLD [367], RUS-EUR: ARC [47, 127, 150], NW [207, 212, 364, 407], NE [207, 212, 415], KLN [309], C [207, 212], N-UR [150, 207, 212], S-UR [150, 207, 212], SE [207, 212], CAUC [55, 212, 231, 248], GEO [117], ARM [297], AZE [277], KAZ [296, 356], UZB [296], KYR [296], RUSAS: ARC-WSIB [136], ARC-ESIB [164, 212], W-SIB [212, 265], S-SIB [69, 70, 212, 219], E-SIB $[69,212]$, ARC-YAK $[47,212,239]$, YAK [212, 239], ARC-FE [40, 212], N-FE [94, 140], S-FE $[76,109,212]$

- strictum Brid. (P. affine Funck, P. alpestre Hoppe) - LTV [244], LAT [3], EST [237], BEL [355], UKR: C [66], M [66], RUS-EUR: ARC [47, 150], NW [207, 212, 364, 407], NE [207, 212, 415], KLN [309], C [207, 212], N-UR [150, 207, 212], S-UR [150, 207, 212], SE [207], CAUC [55, 212, 231, 248], AZE [277], KAZ [296, 356], TAD [295], RUS-AS: ARC-WSIB [136], ARC-ESIB [164, 212], W-SIB [212, 265], S-SIB [69, 70, 212, 219], E-SIB [69, 212], ARC-YAK [47, 212, 239], YAK [212, 239], ARC-FE [40, 212], N-FE [94, 140], S-FE [76, 77, 109, 212]

- swartzii Hartm. (P. commune var. swartzii (Hartm.) Nyholm) - LAT [1], BEL [355], RUS-EUR: ARC [47, 150], NW [207, 212, 262, 364, 407], NE [53, 101, 207], C [207, 212, 345], N-UR [150], S-UR [150], CAUC [248], RUS-AS: ARC-ESIB [212, 263], W-SIB [265], ARC-YAK [47, 239], YAK [239], ARC-FE [40], N-FE [40, 140], S-FE [109, 212] 
Pottia see Tortula, Microbryum, Hennediella heimii

Pseudephemerum (Lindb.) I.Hagen [Ditrichaceae]

- nitidum (Hedw.) Loeske - LTV [244], LAT [5], EST [237], BEL [355], UKR: C [66], M [66]), RUSEUR: NW [207, 407], KLN [309], C [207, 212, 345], RUS-AS: W-SIB [146], S-SIB [336]

Pseudobryum (Kindb.) T.J.Kop. [Mniaceae]

- cinclidioides (Huebener) T.J.Kop. - LTV [244], LAT [3], EST [237], BEL [355], UKR: C [66], M [66], RUS-EUR: ARC [47, 127, 150], NW [207, 212, 364, 407], NE [207, 212, 415], KLN [309], C [207, 212, 345], N-UR [150, 207, 212], S-UR [150, 207, 212], CAUC [231, 248], GEO [117], KYR [296], RUS-AS: ARC-WSIB [136], ARC-ESIB [164, 212], W-SIB [212, 265], S-SIB [69, 70, 212], ESIB [69, 212], ARC-YAK [47, 239], YAK [212, 239], ARC-FE [40, 212], N-FE [94, 140], S-FE [77, $109,212]$

Pseudocalliergon (Limpr.) Loeske [Amblystegiaceae]

— angustifolium Hedenäs - RUS-EUR: NW [391], RUS-AS: YAK [239], N-FE [40]

- brevifolium (Lindb.) Hedenäs (Drepanocladus brevifolius (Lindb.) Warnst., D. latifolius (Lindb. \& Arnell) Warnst.) - RUS-EUR: ARC [47, 150], RUS-AS: ARC-WSIB [136], ARC-ESIB [164, 212, 275], E-SIB [126, 212], ARC-YAK [47, 212, 239], YAK [212, 239], ARC-FE [40, 212], N-FE [40]

- lycopodioides (Brid.) Hedenäs (Drepanocladus lycopodioides (Brid.) Warnst.) - LTV [244], LAT [3], EST [237], BEL [355], UKR: C [66], M [66], RUS-EUR: NW [364, 407], NE [101], KLN [309], C [207], N-UR [150, 415], CAUC [55, 212], GEO [117], RUS-AS: W-SIB [338]

- trifarium (F.Weber \& D.Mohr) Loeske (Calliergon trifarium (F.Weber \& D.Mohr) Kindb.) - LTV [244], LAT [3], EST [237], BEL [355], UKR: M [66], RUS-EUR: NW [207, 212, 364, 407], NE [207, 212, 415], KLN [309], C [207], N-UR [90], S-UR [151], ARM [297], KAZ [296, 356], RUSAS: ARC-WSIB [136], ARC-ESIB [212, 275], W-SIB [265], S-SIB [69, 70, 212], E-SIB [163], ARCYAK [47, 239], YAK [212, 239], ARC-FE [40, 212], N-FE [40, 94, 275]

- turgescens (T.Jensen) Loeske (Scorpidium turgescens (T.Jensen) Loeske) - LAT [4], EST [237], RUSEUR: ARC [47, 150], NW [85, 212], KLN [309], TAD [296], KYR [296], RUS-AS: ARC-WSIB [136], ARC-ESIB [164, 212, 275], S-SIB [69, 70, 197], E-SIB [126, 212], ARC-YAK [47, 212, 239], YAK [212, 239], ARC-FE [40, 212], N-FE [40, 140]

Pseudocrossidium R.S.Williams [Pottiaceae]

- hornschuchianum (Schultz) R.H.Zander (Barbula hornschuchiana Schultz) - LTV [244], EST [237], UKR: C [66], M [66], K [328], MLD [367], RUS-EUR: KLN [309], SE [165, 207, 212], GEO [117], ARM [297], AZE [277], TUR [296], TAD [294, 295], KYR [296] - \{152\}

- obtusulum (Lindb.) H.A.Crum \& L.E.Anderson - RUS-EUR: S-UR [165], SE [165, 366], RUS-AS: E-SIB [165], YAK [165]

- revolutum (Brid.) R.H.Zander (Barbula revoluta Brid.) - EST [397], UKR: M [66], K [66, 328], MLD [367], GEO [117], TUR [296], TAD [294, 295] — \{152\}

Pseudohygrohypnum Kanda [Pylaisiaceae]

- eugyrium (Bruch et al.) Kanda (Hygrohypnum eugyrium (Bruch et al.) Broth.) - RUS-AS: S-FE [138, 76]

- subeugyrium (Renauld \& Cardot) Ignatov \& Ignatova (Hygrohypnum subeugyrium (Renauld \& Cardot) Broth.) - RUS-EUR: S-UR [138], RUS-AS: ARC-ESIB [164], S-SIB [138], E-SIB [138], YAK $[138,239], \mathrm{N}-\mathrm{FE}$ [138], S-FE [138, 221]

Pseudoleskea see Lescuraea

Pseudoleskeella Kindb. [Pseudoleskeellaceae]

- catenulata (Brid. ex Schrad.) Kindb. - LTV [244], LAT [5], EST [237], UKR: C [66], M [66], K [66, 328], RUS-EUR: ARC [47, 150], NW [364], C [207, 212, 345], N-UR [150, 207, 415], S-UR [150, 207, 212], SE [207], CAUC [55, 212, 231, 248], GEO [117], ARM [297], KAZ [156, 296, 356], TAD [294, 296], KYR [296, 267, 349, 351], RUS-AS: ARC-ESIB [164, 212], S-SIB [69, 70, 212], E-SIB [212, 247], ARC-YAK [47], YAK [212, 239], ARC-FE [40, 212], N-FE [40], S-FE [76, 109, 212]

- nervosa (Brid.) Nyholm (Leskeella nervosa (Brid.) Loeske, L incrassata (Lindb. ex Broth.) Broth.) LTV [244], LAT [3], EST [237], BEL [355], UKR: C [66], M [66], K [66, 328], MLD [367], RUSEUR: ARC [47, 150], NW [207, 212, 364, 407], NE [207, 212, 415], KLN [309], C [207, 212], N-UR [150, 207, 212], S-UR [150, 207, 212], SE [207, 212], CAUC [55, 212, 231, 248], GEO [117], ARM 
[297], AZE [277], KAZ [156, 296, 356], KYR [296, 351], RUS-AS: ARC-WSIB [136], W-SIB [265], S-SIB [69, 70, 212], E-SIB [69, 212], ARC-YAK [47, 239], YAK [212, 239], ARC-FE [40, 212], N-FE [94, 140], S-FE [76, 77, 109, 212] - $\{153\}$

- papillosa (Lindb.) Kindb. (Heterocladium papillosum (Lindb.) Lindb.) - RUS-EUR: NW [85, 212, 364, 407], N-UR [150, 207, 212], S-UR [150, 207], KYR [267, 296], RUS-AS: ARC-WSIB [136], ARC-ESIB [164, 212], S-SIB [69, 212], E-SIB [70, 212, 275], ARC-YAK [47, 239], YAK [239], ARC-FE [40], N-FE [94, 140], S-FE [212, 221]

- rupestris (Berggr.) Hedenäs \& L.Söderstr. (P. nervosa var. rupestris (Berggr.) Nyholm, P. sibirica (Arnell) P.S.Wilson \& D.H.Norris) - UKR [66], RUS-EUR: N-UR [432], S-UR [207], KAZ [156], KYR [351], RUS-AS: ARC-ESIB [164], S-SIB [70, 212], E-SIB [275, 388], ARC-YAK [47, 239], YAK [239]

- tectorum (Funck ex Brid.) Kindb. ex Broth. - UKR: C [66], M [66], K [66, 328], RUS-EUR: ARC [150, 415], NW [212, 273, 364, 407], C [207, 212], N-UR [150, 415], S-UR [150, 207, 212], SE [207], CAUC [55, 212, 231, 248], GEO [117], ARM [297], AZE [277], KAZ [23, 156, 296, 356], TUR [296], TAD [294, 296], KYR [267, 296, 349, 351], RUS-AS: ARC-WSIB [136], ARC-ESIB [164, 212], W-SIB [265], S-SIB [69, 70, 212], ARC-YAK [47, 239], YAK [212, 239], ARC-FE [40, 212], NFE [94, 140], S-FE [76, 212, 221, 268]

Pseudoleskeopsis Broth. [Leskeaceae]

- zippelii (Dozy \& Molk.) Broth. - RUS-AS: S-FE [268]

Pseudoscleropodium (Limpr.) M.Fleisch. [Brachytheciaceae]

- purum (Hedw.) M.Fleisch. ex Broth. (Scleropodium purum (Hedw.) Limpr.) - LTV [244], LAT [3], EST [237], BEL [355], UKR: C [66], M [66], K [66, 328], RUS-EUR: ?ARC [415], NW [207], KLN [309], C [207, 212], SE [207], CAUC [208, 212, 248], GEO [117], ARM [297], AZE [277], ?KAZ [296]

Pseudotaxiphyllum Z.Iwats. [*Plagiotheciaceae]

- elegans (Brid.) Z.Iwats. (Isopterygium elegans (Brid.) Lindb.) - UKR: C [66], M [66], RUS-EUR: NW [407], CAUC [55, 212], GEO [117], RUS-AS: ARC-FE [40, 212], N-FE [94, 140], S-FE [111, 212]

Psilopilum Brid. [Polytrichaceae]

- cavifolium (Wilson) I.Hagen - RUS-EUR: ARC [47, 150, 415], NW [364], NE [415], N-UR [150, 415], RUS-AS: ARC-WSIB [136], ARC-ESIB [164, 212], E-SIB [163], ARC-YAK [47, 212, 239], YAK [239], ARC-FE [40, 212], N-FE [94]

- laevigatum (Wahlenb.) Lindb. - RUS-EUR: ARC [47, 127, 150, 415], NW [364], N-UR [150], RUSAS: ARC-WSIB [136], ARC-ESIB [164, 212], E-SIB [74, 212], ARC-YAK [47, 212, 239], YAK [239], ARC-FE [40], N-FE [140] - \{154\}

Pterigynandrum Hedw. [Pterigynandraceae]

- filiforme Hedw. - LTV [244], LAT [3], EST [237], BEL [355], UKR: C [66], M [66], K [66, 328], RUS-EUR: ARC [47, 150], NW [207, 212, 364, 407], KLN [309], C [207, 212, 345], N-UR [90, 150, 207, 212, 415], S-UR [150, 207, 212], SE [207], CAUC [55, 208, 212, 231, 248], GEO [117], ARM [297], AZE [277], KAZ [156, 159, 296, 356], KYR [296], RUS-AS: ARC-ESIB [164, 212], S-SIB [69, 70, 212], E-SIB [68, 212], ARC-YAK [47, 239], YAK [239], ARC-FE [40, 212], N-FE [94, 140], S-FE [76, 77, 212]

Pterogonium Sw. [Pterogoniaceae]

— gracile (Hedw.) Sm. - EST [237], UKR: K [66, 328], ARM [297], AZE [277]

Pterygoneurum Jur. [Pottiaceae]

— kozlovii Laz. - UKR: M [66], MLD [367], RUS-EUR: SE [207], KAZ [337], RUS-AS: W-SIB [337], YAK $[239,337]-\{155\}$

- lamellatum (Lindb.) Jur. - UKR: M [66], RUS-EUR: SE [207, 212, 377], GEO [102, 117], AZE [277], TUR [267, 296], UZB [267, 295], TAD [295], RUS-AS: ARC-ESIB [47], E-SIB [163], ARCFE $[40]-\{156\}$

- ovatum (Hedw.) Dixon - LTV [244], LAT [3], BEL [355], UKR: C [66], M [66], K [66, 328], MLD [367], RUS-EUR: NW [207], C [207, 212, 345], S-UR [150, 207], SE [207, 212], CAUC [232, 248], GEO [117], ARM [297], AZE [277], KAZ [157, 296, 349], TUR [11, 295, 296], UZB [267, 295, 296], TAD [294, 295], KYR [296, 351], RUS-AS: ARC-ESIB [39, 50], W-SIB [337], S-SIB [197], ESIB [162], YAK [239], ARC-FE [40, 212] 
- subsessile (Brid.) Jur. - LTV [244], UKR: C [66], M [66], K [328], MLD [367], RUS-EUR: KLN [309], C [207, 345], S-UR [150, 207], SE [207, 212], CAUC [421], GEO [117], AZE [277], KAZ [296], TUR [295, 296], UZB [295], TAD [267, 295], KYR [296, 349, 351], RUS-AS: W-SIB [265], S-SIB [69, 70, 212], E-SIB [126, 212], YAK [239]

Ptilium De Not. [Pylaisiaceae]

- crista-castrensis (Hedw.) De Not. - LTV [244], LAT [3], EST [237], BEL [355], UKR: C [66], M [66], RUS-EUR: ARC [47, 127, 150], NW [207, 212, 364, 407], NE [207, 212, 415], KLN [309], C [207, 212], N-UR [150, 207, 212], S-UR [150, 207, 212], SE [207, 212], CAUC [55, 212, 231, 248], GEO [117], ARM [297], AZE [277], KAZ [156, 296, 356], TAD [26], KYR [296], RUS-AS: ARCWSIB [136], ARC-ESIB [212], W-SIB [212, 265], S-SIB [69, 70, 212], E-SIB [69, 212, 275], ARCYAK [47, 239], YAK [212, 239], ARC-FE [40], N-FE [94, 140], S-FE [76, 77, 109, 212]

Ptychodium see Lescuraea

Ptychomitrium Fürnr. [Ptychomitriaceae]

— incurvum (Schwägr.) Spruce - GEO [117]

— sinense (Mitt.) A.Jaeger - RUS-AS: S-SIB [69, 70, 197], S-FE [76, 212]

Pylaisia Bruch et al. [Pylaisiaceae]

— brotheri Besch. - RUS-AS: S-FE [76, 77, 212]

- curviramea Dixon - RUS-AS: S-FE [64] - $\{157\}$

- obtusa Lindb. - RUS-AS: S-FE [109]

- polyantha (Hedw.) Bruch et al. (Pylaisiella polyantha (Hedw.) Grout) - LTV [244], LAT [3], EST [237], BEL [355], UKR: C [66], M [66], K [66, 328], MLD [367], RUS-EUR: ARC [47, 150], NW [207, 212, 364, 407], NE [207, 212, 415], KLN [309], C [207, 212], N-UR [150, 207, 212], S-UR [150, 207, 212], SE [207, 212], CAUC [55, 208, 212, 231, 248], GEO [117], ARM [297], AZE [277], KAZ [159, 296, 356], RUS-AS: W-SIB [212, 265], S-SIB [69, 70, 212], E-SIB $[69,162,212,275]$, ARC-YAK [47, 239], YAK [212, 239], ARC-FE [40], N-FE [94, 140], S-FE $[76,77,109,212]$

- selwynii Kindb. (Pylaisiella selwynii (Kindb.) H.A.Crum, Steere \& L.E.Anderson) - RUS-EUR: NW [207, 407], NE [90, 207, 212, 366a], C [207, 212], S-UR [150, 207, 212], RUS-AS: W-SIB [212, 265], S-SIB [69, 70, 212], E-SIB [212, 247], YAK [239], N-FE [94, 140], S-FE [76, 77, 109, 212]

— steerei (Ando \& Higuchi) Ignatov - RUS-AS: E-SIB [387], YAK [239]

- stereodontoides Broth. \& M.Yasuda ex Iishiba (P. intricata auct. non (Hedw.) Bruch et al.) - RUS-AS: S-FE $[76,178,212]-\{158\}$

- subcircinata Cardot - RUS-AS: N-FE [140], S-FE [76, 77, 109, 212]

Pylaisiadelpha Cardot [Pylaisiadelphaceae]

— tenuirostris (Bruch \& Schimp. ex Sull.) W.R.Buck - RUS-AS: S-SIB [69], E-SIB [212, 247], S-FE $[76,114,212,221]-\{159\}$

Pylaisiella see Pylaisia

Pyramidula Brid. [Funariaceae]

— tetragona (Brid.) Brid. - UKR: M [66], RUS-EUR: C [207, 345], S-UR [150, 207], AZE [277], RUSAS: S-SIB [69]

Racomitrium Brid. [Grimmiaceae], see also Bucklandiella heterosticha, etc., Niphotrichum canescens, etc., Codriophorus fascicularis, etc.

- lanuginosum (Hedw.) Brid. - LAT [3], EST [237], UKR: C [66], RUS-EUR: ARC [47, 127, 150], NW [207, 212, 364, 407], KLN [309], N-UR [150, 207, 212, 415], S-UR [150], CAUC [212, 248], AZE [277], RUS-AS: ARC-WSIB [136], ARC-ESIB [164, 212], S-SIB [69, 70, 202, 212], E-SIB [69, 212], ARC-YAK [47, 212, 239], YAK [239], ARC-FE [40, 212], N-FE [94, 140], S-FE [76, 77, 109, 212]

Rauiella Reimers [Thuidiaceae]

- fujisana (Paris) Reimers - RUS-AS: N-FE [140], S-FE [76, 77, 109, 212]

Rhabdoweisia Bruch et al. [Rhabdoweisiaceae]

- crispata (Dicks. ex With.) Lindb. (R. kusenevae Broth.) - LAT [3], UKR: C [66], RUS-EUR: N-UR [150, 207, 212], S-UR [150, 207, 212], RUS-AS: S-SIB [69, 70, 212], E-SIB [69, 163, 212], YAK [239], ARC-FE [40], N-FE [94, 140], S-FE [76, 77, 109, 212] 
- $\operatorname{fugax}$ (Hedw.) Bruch et al. - LAT [3], UKR: C [66], M [66], K [66, 328], RUS-EUR: ARC [47, 150], NW [87, 364, 407], N-UR [150], CAUC [55, 102, 212, 231], GEO [18, 117]

Rhizomnium (Broth.) T.J.Kop. [Mniaceae]

- andrewsianum (Steere) T.J.Kop. - RUS-EUR: ARC [47, 127, 150], NW [364], N-UR [150, 370, 415], KAZ [296, 356], RUS-AS: ARC-WSIB [136], ARC-ESIB [164, 212], S-SIB [69, 70, 197, 212], E-SIB [69, 212], ARC-YAK [47, 212, 239], YAK [239], ARC-FE [40, 212], N-FE [94, 140], S-FE $[212,221]$

- gracile T.J.Kop. - RUS-AS: ARC-FE [40], N-FE [40, 140]

- magnifolium (Horik.) T.J.Kop. (R. punctatum var. elatum (Schimp.) T.J.Kop.) - EST [396], UKR: C [401], RUS-EUR: ARC [47, 127, 150], NW [207, 212, 364, 407], NE [207, 212, 415], C [207, 212], N-UR [150, 207], S-UR [150, 207, 212], GEO [117], RUS-AS: S-SIB [70, 212], E-SIB [212, 247], YAK [239], ARC-FE [40], N-FE [94, 140], S-FE [77, 109, 212]

— nudum (E.Britton \& R.S.Williams) T.J.Kop. - RUS-AS: E-SIB [212, 247], N-FE [94, 110, 140], S-FE [67]

— parvulum (Mitt.) T.J.Kop. - RUS-AS: S-FE [76, 212]

— pseudopunctatum (Bruch \& Schimp.) T.J.Kop. - LTV [244], LAT [3], EST [237], UKR: C [66], RUS-EUR: ARC [47, 127, 150], NW [207, 212, 364, 407], NE [207, 212, 415], KLN [309], C [207, 212], N-UR [150, 207, 212], S-UR [150, 207, 212], SE [207], CAUC [55, 212, 231, 248], GEO [117], KAZ [159, 296], KYR [296], RUS-AS: ARC-WSIB [136], ARC-ESIB [96, 212], W-SIB [212, 265], S-SIB [69, 70, 212], E-SIB [69, 212], ARC-YAK [47, 212, 239], YAK [239], ARC-FE [40, 212], N-FE [94, 140], S-FE [109, 212, 221]

— punctatum (Hedw.) T.J.Kop. - LTV [244], LAT [3], EST [237], BEL [355], UKR: C [66], M [66], K [66, 328], MLD [367], RUS-EUR: ARC [47, 150], NW [207, 212, 364, 407], NE [207, 212, 415], KLN [309], C [207, 212], N-UR [150, 207, 212], S-UR [150, 207, 212], SE [207, 212], CAUC [55, 208, 212, 231, 248], GEO [117], ARM [297], AZE [277], KAZ [356], RUS-AS: ARC-ESIB [212], W-SIB [212, 265], S-SIB [69, 70, 212], E-SIB [69, 212], ?ARC-YAK [47, 212], ?N-FE [140], ?S-FE $[109,212]-\{160\}$

— striatulum (Mitt.) T.J.Kop. - RUS-AS: N-FE [140], S-FE [76, 77, 212]

- tuomikoskii T.J.Kop. - RUS-AS: N-FE [254]

Rhodobryum (Schimp.) Limpr. [Bryaceae]

— ontariense (Kindb.) Kindb. - LAT [3], EST [237], UKR: C [329], M [329], K [328], RUS-EUR: N-UR [91], S-UR [91, 207], CAUC [212, 248], ARM [297], RUS-AS: S-SIB [197, 212], S-FE [79, 212]

- roseum (Hedw.) Limpr. - LTV [244], LAT [3], EST [237], BEL [355], UKR: C [66], M [66], K [66, 328], MLD [367], RUS-EUR: ARC [47, 150], NW [207, 212, 364, 407], NE [207, 212, 415], KLN [309], C [207], N-UR [150, 207, 212], S-UR [150, 207, 212], SE [207], CAUC [55, 212, 231, 248], GEO [117], ARM [297], AZE [277], KAZ [156, 159, 296], KYR [296], RUS-AS: WSIB [212, 265], S-SIB [69, 70, 212], E-SIB [69, 212], YAK [239], N-FE [94, 140], S-FE [109, $221]-\{161\}$

Rhynchostegiella (Schimp.) Limpr. [Brachytheciaceae], see also Oxyrrynchium pumilum (=Rhynchostegiella pallidirostris)

- curviseta (Brid.) Limpr. - RUS-EUR: CAUC [232], TAD [294, 296]

- tenella (Dicks.) Limpr. - UKR: M [66], K [66, 328], RUS-EUR: CAUC [208, 212, 232, 248], GEO [117], ARM [297], AZE [277], KAZ [296, 393]

- teneriffae (Mont.) Dirkse \& Bouman (R. jacquinii (Garov.) Limpr., R. teesdalei (Bruch et al.) Limpr.) - RUS-EUR: CAUC [208, 212, 232, 248], GEO [31, 102], ARM [297], AZE [277]

Rhynchostegium Bruch et al. [Brachytheciaceae]

- arcticum (I.Hagen) Ignatov \& Huttunen (R. murale var. arcticum I.Hagen, Scleropodiopsis laxiretis Ignatov) - RUS-EUR: NE [415], C [207], N-UR [207], S-UR [207, 212], RUS-AS: S-SIB [198]

- confertum (Dicks.) Bruch et al. - BEL [355], RUS-EUR: CAUC [55, 212, 232], GEO [117], AZE [277], RUS-AS: ?S-FE [268] - $\{162\}$

- megapolitanum (Blandow ex F.Weber \& D.Mohr) Bruch et al. - UKR: C [66], K [66, 328], MLD [367], RUS-EUR: KLN [309], CAUC [6, 232], GEO [117], ARM [297], AZE [277], TUR [296, 9]

- murale (Hedw.) Bruch et al. - LTV [244], LAT [3], EST [237], BEL [355], UKR: C [66], M [66], K 
[66, 328], MLD [367], RUS-EUR: NW [63], KLN [309], C [207, 212, 345], NE [415], CAUC [55, 212, 232, 248], AZE [277]

— pallidifolium (Mitt.) A.Jaeger - RUS-AS: S-FE [178]

- riparioides (Hedw.) Cardot (Platyhypnidium riparioides (Hedw.) Dixon) - LTV [244], LAT [3], EST [237], BEL [355], UKR: C [66], M [66], K [66, 328], RUS-EUR: NW [207, 364, 407], NE [207, 212 , 366a], KLN [309], C [207, 345], N-UR [207, 416], S-UR [207, 212], SE [207], CAUC [55, 208, 212, 231, 248], GEO [117], ARM [297], AZE [277], TUR [267, 296], KAZ [156, 159], UZB [347], TAD [294, 296], KYR [296, 351], RUS-AS: S-SIB [69, 70, 198, 212], E-SIB [69], YAK [239], S-FE [76, $77,109,212]-\{163\}$

- rotundifolium (Scop. ex Brid.) Bruch et al. - UKR: C [66], RUS-EUR: S-UR [207, 212], SE [207], CAUC [55, 212, 232, 248], GEO [117], AZE [277], RUS-AS: S-FE [109]

Rhytidiastrum see Rhytidiadelphus

Rhytidiadelphus (Limpr.) Warnst. [Hylocomiaceae] $-\{164\}$

- japonicus (Reimers) T.J.Kop. - RUS-AS: S-FE [76, 77, 114, 212]

- loreus (Hedw.) Warnst. - LTV [244], EST [397], UKR: C [66, 413], RUS-EUR: KLN [309]

- squarrosus (Hedw.) Warnst. (Rhytidiastrum squarrosum (Hedw.) Ignatov \& Ignatova) - LTV [244], LAT [3], EST [237], BEL [355], UKR: C [66], M [66], RUS-EUR: ARC [47], NW [207, 212, 364, 407], NE [207, 212, 415], KLN [309], C [207, 212], N-UR [150, 207], S-UR [150, 207, 212], CAUC [55, 212, 248], GEO [117], ARM [297], AZE [277], KAZ [?296], RUS-AS: S-SIB [70, 212], ARCFE [40], N-FE [140], S-FE [109]

- subpinnatus (Lindb.) T.J.Kop. (R. squarrosus var. calvescens (Kindb.) Warnst., Rhytidiastrum subpinnatum (Lindb.) Ignatov \& Ignatova) - LTV [244], LAT [3], EST [237], BEL [?355], UKR: C [66], RUS-EUR: ARC [47, 127, 150], NW [207, 212, 364, 407], NE [207, 212, 415], C [207, 212], N-UR [150, 207, 212], S-UR [150, 207, 212], CAUC [248], ARM [297], RUS-AS: ARC-WSIB [?47], ARCESIB [?47], W-SIB [212, 265], S-SIB [69, 70, 212], E-SIB [212, 275], YAK [239], ARC-FE [40], NFE [94, 140], S-FE [77, 109, 212]

— triquetrus (Hedw.) Warnst. - LTV [244], LAT [3], EST [237], BEL [355], UKR: C [66], M [66], K [66, 328], MLD [367], RUS-EUR: ARC [47, 127, 150], NW [207, 212, 364, 407], NE [207, 212, 415], KLN [309], C [207, 212], N-UR [150, 207, 212], S-UR [150, 207, 212], SE [207, 212], CAUC [55, 212, 231, 248], GEO [117], ARM [297], AZE [277], KAZ [156, 296, 356], KYR [296, 351], RUS-AS: ARCWSIB [136], ARC-ESIB [275], W-SIB [212, 265], S-SIB [69, 70, 212], E-SIB [69, 212, 275], ARCYAK [47, 239], YAK [212, 239], ARC-FE [40], N-FE [94, 140], S-FE [76, 77, 109, 212]

Rhytidium (Sull.) Kindb. [Rhytidiaceae]

- rugosum (Hedw.) Kindb. - EST [237], UKR: C [66], M [66], K [66, 328], RUS-EUR: ARC [47, 127, 150], NW [87, 212, 364, 407], NE [207, 212, 415], C [207, 212, 345], N-UR [150, 207, 212], S-UR [150, 207, 212], CAUC [55, 212, 231, 248], GEO [117], ARM [297], AZE [277], KAZ [156, 159, 296, 356], KYR [351], RUS-AS: ARC-WSIB [136], ARC-ESIB [164, 212], W-SIB [265], S-SIB [69, 70, 212], E-SIB [69, 212, 275], ARC-YAK [47, 212, 239], YAK [212, 239], ARC-FE [40, 212], N-FE [94, 140], S-FE [76, 77, 109, 212]

Rigodiadelphus Dixon [Pseudoleskeaceae]

— robustus (Lindb.) Nog. - RUS-AS: N-FE [140], S-FE [76, 77, 109, 212]

Saelania Lindb. [Ditrichaceae]

- glaucescens (Hedw.) Broth. - LAT [3], EST [237], UKR: C [66], K [66, 328], RUS-EUR: ARC [47, 150], NW [207, 212, 364, 407], NE [207, 212, 415], C [207], N-UR [150, 207, 212], S-UR [150, 207, 212], CAUC [55, 102, 212, 231, 248], GEO [117], AZE [277], KAZ [104, 159], KYR [104, 296, 349, 351], RUS-AS: ARC-WSIB [136], ARC-ESIB [164, 212], W-SIB [212, 265], S-SIB [69, 70, 212], E-SIB [69, 104, 212, 275], ARC-YAK [47, 239], YAK [239], ARC-FE [40, 212], N-FE [94, 140], S-FE [76, 77, 109, 212]

Sanionia Loeske [Scorpidiaceae]

— georgicouncinata (Müll.Hal.) Ochyra \& Hedenäs (Sanionia nivalis Hedenäs) - RUS-EUR NW [184], RUS-AS: ARC-ESIB [50, 39], ARC-YAK [47, 239]

— orthothecioides (Lindb.) Loeske - RUS-EUR: NW [84, 291], RUS-AS: ARC-ESIB [212, 263], ARCFE [40] 
- uncinata (Hedw.) Loeske (Drepanocladus uncinatus (Hedw.) Warnst.) - LTV [244], LAT [3], EST [237], BEL [355], UKR: C [66], M [66], K [66, 328], MLD [367], RUS-EUR: ARC [47, 127, 150], NW [207, 212, 364, 407], NE [207, 212, 415], KLN [309], C [207, 212], N-UR [150, 207, 212], S-UR [150, 207, 212], SE [207, 212], CAUC [55, 212, 231, 248], GEO [117], ARM [297], AZE [277], KAZ [156, 296, 356], TAD [26, 296], KYR [296, 351], RUS-AS: ARC-WSIB [136], ARC-ESIB [164, 212], W-SIB [212, 265], S-SIB [69, 70, 212], E-SIB [69, 212, 275], ARC-YAK [47, 212, 239], YAK [212, 239], ARC-FE [40, 212], N-FE [94, 140], S-FE [76, 77, 109, 212]

Sarmentypnum see Warnstorfia $-\{165\}$

Sasaokaea Broth. [Amblystegiaceae]

- aomoriensis (Paris) Kanda - RUS-AS: S-FE [178]

Schistidium Bruch et al. [Grimmiaceae]

— agassizii Sull. \& Lesq. - LAT [3], EST [237], RUS-EUR: ARC [150, 415], NW [212, 273, 364, 407], NE [207, 212, 415], N-UR [150, 207, 415], S-UR [150, 207], CAUC [55, 212, 231, 248], KAZ [159, 296, 356], TAD [296], KYR [296], RUS-AS: ARC-ESIB [164, 212], S-SIB [70, 197, 202, 212], E-SIB [74, 212], ARC-YAK [47, 239], YAK [212, 239], ARC-FE [40], N-FE [94, 140], S-FE [76, 212, 221]

- andreaeopsis (Müll.Hal.) Laz. - RUS-EUR: ARC [99], RUS-AS: ARC-ESIB [47, 212], ARC-YAK $[47,239]$, ARC-FE [40, 212]

- apocarpum (Hedw.) Bruch et al. - LTV [97, 244], LAT [3, 97], EST [97, 237], BEL [355], UKR: C [66], M [66, 97], K [66], RUS-EUR: ARC [150], NW [97, 207, 273, 364, 407], NE [97, 207, 415], KLN [309], C [97, 207], N-UR [150, 207], S-UR [150, 207], SE [207], CAUC [55, 208, 231, 232, 248], GEO [97, 117], ARM [97, 297], AZE [97, 277], KAZ [356], TAD [294], KYR [351], RUS-AS: ARC-WSIB [136], W-SIB [265], S-SIB [70, 97, 202], N-FE [94, 140], S-FE [76, 77] — \{166\}

- apocarpum subsp. canadense (Dupret) H.H.Blom ex B.H. Allen \& Pursell - RUS-AS: S-SIB [99], ESIB [99], S-FE [99]

- boreale Poelt - RUS-EUR: NW [97, 282], C [207], N-UR [207], S-UR [97, 207], RUS-AS: S-SIB [97], YAK [239], ARC-FE [99]

- brunnescens Limpr. - UKR: M [66, 97], K [66], MLD [97], GEO [97, 117], TUR [267, 296], KYR [296], UZB [267], RUS-AS: S-FE [76]

- brunnescens subsp. griseum (Nees \& Hornsch.) H.H.Blom - RUS-EUR: SE [366]

- confertum (Funck) Bruch et al. (S. apocarpum var. confertum (Funck) H.Möller) - EST [237], UKR: C [66], M [66], K [66], MLD [367], GEO [117, 97], ARM [97], AZE [277], RUS-AS: ?NW [364], ?S-SIB [70], ?YAK [239], ?N-FE [94], ?S-FE [76] - \{167\}

- confusum H.H.Blom - LAT [3, 97], EST [237, 97], RUS-EUR: NW [99, 233]

- crassipilum H.H.Blom - LAT [3, 97], EST [97, 396], UKR: M, K [97], RUS-EUR: KLN [309], S-UR [207, 212], SE [366], CAUC [231, 232], GEO [97], ARM [97]

- crenatum H.H.Blom - RUS-EUR: NW [98, 233], RUS-AS: S-SIB [98]

- cryptocarpum Mogensen \& H.H.Blom - RUS-AS: ARC-FE [40, 51], N-FE [40, 141]

- dupretii (Thér.) W.A.Weber - RUS-EUR: NW [97, 233], NE [414], C [207, 97], N-UR [207], S-UR [212, 422], SE [99, 366], CAUC [231, 248], GEO [97], KAZ [97], RUS-AS: S-SIB [212, 336], YAK [239], N-FE [140]

- elegantulum H.H.Blom - EST [396], RUS-EUR: NW [233], SE [207], CAUC [212, 231, 248, 208], GEO [97], AZE [97], RUS-AS: S-SIB [97], S-FE [99]

- flaccidum (De Not.) Ochyra (S. pulvinatum var. flaccidum (De Not.) De Not.) - UKR: M [66], K [328], RUS-EUR: NW [97, 407], S-UR [99], SE [366], CAUC [231, 248, 102], GEO [117, 97], ARM [297, 97], AZE [97], TAD [294], KYR [349, 351], UZB [267], RUS-AS: S-SIB [99]

- flexipile (Lindb. ex Broth.) G.Roth - RUS-EUR: ARC [97], NW [97, 233], GEO [97, 102], RUS-AS: ARC-YAK [239], ?ARC-FE [40]

- frigidum H.H.Blom - RUS-EUR: NW [97, 233, 282], N-UR [97, 99], RUS-AS: ARC-ESIB [164, 212], S-SIB [99], E-SIB [50, 99], ARC-YAK [239], ARC-FE [40, 97], N-FE [40, 140]

- frisvollianum H.H.Blom - RUS-EUR: NW [97, 233], RUS-AS: ARC-ESIB [39, 164], E-SIB [163], ARC-YAK [97, 239], YAK [239], ARC-FE [40] 
— grandirete H.H.Blom - RUS-AS: ARC-ESIB [50, 97], E-SIB [163], ARC-YAK [239], ARC-FE [40, 97]

- helveticum (Schkuhr) Deguchi (Schistidium singarense (Schiffn.) Laz.) - RUS-EUR: SE [366], GEO [97], KAZ [157], TUR [296], KYR [267, 296]

- holmenianum Steere \& Brassard - RUS-AS: ARC-ESIB [50], ARC-YAK [239], ARC-FE [40]

— lancifolium (Kindb.) H.H.Blom - RUS-EUR: NW [97, 233], C [97], S-UR [212, 422], CAUC [231], GEO [97], RUS-AS: S-SIB [99], N-FE [141], S-FE [97]

- liliputanum(Müll.Hal.) Deguchi - RUS-AS: S-SIB [202, 212], S-FE [212, 221]

- maritimum (Sm. ex R.Scott) Bruch et al. - EST [237], RUS-EUR: ARC [42, 47], NW [233, 364], KLN [309], RUS-AS: N-FE [140], S-FE [77, 114]

— maritimum subsp. piliferum (I.Hagen) B.Bremer - EST [396], RUS-EUR: NW [98, 233)]

— papillosum Culm. (S. apocarpum subsp. papillosum (Culm.) Poelt) - LAT [97], EST [397], UKR: C [66], M [66], RUS-EUR: ARC [97], NW [97, 233, 282], N-UR [207], S-UR [207], CAUC [231, 248], GEO [97], KAZ [97], RUS-AS: ARC-ESIB [39, 164], S-SIB [97], E-SIB [163], ARC-YAK [239], YAK [239], ARC-FE [40, 97], N-FE [140], S-FE [97, 114]

- platyphyllum (Mitt.) Perss. (S. alpicola auct. non (Hedw.) Limpr., S. rivulare subsp. latifolium (J.E.Zetterst.) B.Bremer, S. rivulare var. latifolium (J.E.Zetterst.) H.A.Crum \& L.E.Anderson) - RUSEUR: ARC [47, 127], NW [233], NE [?47], RUS-AS: ARC-WSIB [136], ARC-ESIB [39, 164], S-SIB [98], ARC-YAK [47, 239], YAK [239], ARC-FE [40], N-FE [140], S-FE [67]

— platyphyllum subsp. abrupticostatum (Bryhn) H.H.Blom - RUS-EUR: ARC [99], RUS-AS: ARCESIB [50, 98], E-SIB [388]

- pruinosum (Wilson ex Schimp.) G.Roth - RUS-EUR: S-UR [212, 422], CAUC [231, 248], GEO [97], ARM [97]

— pulchrum H.H.Blom - UKR: M [97], RUS-EUR: ARC [99], NW [97, 233], S-UR [212, 422], RUSAS: ARC-ESIB [164, 212], S-SIB [97], E-SIB [97, 388], ARC-YAK [97, 239], YAK [239], ARC-FE [99], N-FE [140], S-FE [97]

- recurvum H.H.Blom - RUS-EUR [233, 282], KAZ [97]

- rivulare (Brid.) Podp. - EST [237], UKR: C [66], M [66], K [66, 328], RUS-EUR: ARC [47, 127, 150], NW [87, 207, 364, 407], NE [207], C [207], N-UR [90, 150, 207], S-UR [150, 207], CAUC [55, 231, 248], GEO [117], KAZ [159, 296, 356], UZB [347], TAD [294, 296], KYR [296, 351], RUSAS: ARC-WSIB [136], ARC-ESIB [164], S-SIB [70, 202, 362], ARC-YAK [47, 239], YAK [239], ARC-FE [40], N-FE [94, 140], S-FE [76, 77, 221]

— robustum (Nees \& Hornsch.) H.H.Blom - LAT [3, 97], EST [97], UKR: M [97], RUS-EUR: NW [97, 233], CAUC [99], GEO [97]

— scandicum H.H.Blom - RUS-EUR: S-UR [99]

— sinensiapocarpum (Müll. Hal.) Ochyra - RUS-EUR: CAUC [99], RUS-AS: S-SIB [99], YAK [99]

— sordidum I.Hagen - RUS-EUR: NW [233], RUS-AS: ARC-ESIB [162], ARC-FE [40]

- strictum (Turner) Loeske ex Mårtensson - excluded - $\{168\}$

- subflaccidum (Kindb.) H.H.Blom - CAUC [99, 231]

— subjulaceum H.H.Blom - RUS-EUR: NW [233], KAZ [97], KYR [97], RUS-AS: S-SIB [97]

- submuticum Broth. ex H.H.Blom - LAT [3], RUS-EUR: NW [233], NE [207], C [97, 207], N-UR [207], S-UR [97, 207], SE [207], RUS-AS: ARC-ESIB [164], S-SIB [99], E-SIB [99], YAK [239]

- submuticum subsp. arcticum H.H.Blom - RUS-AS: ARC-ESIB [164], S-SIB [99], ARC-YAK [97, 239], YAK [97, 239], ARC-FE [99]

- tenerum (J.E.Zetterst.) Nyholm - RUS-EUR: NW [233], RUS-AS: E-SIB [74, 212], ARC-YAK [47, 239], YAK [239], ARC-FE [40, 97, 212], N-FE [40]

— trichodon (Brid.) Poelt - EST [237], GEO [97], ARM [97], KYR [97]

— trichodon var. nutans H.H.Blom - UKR: M [97], RUS-EUR: NW [233], CAUC [212, 231, 248], ARM [297], RUS-AS: S-SIB [97], N-FE [97, 140], S-FE [99]

— umbrosum (J.E. Zetterst.) H.H.Blom - RUS-EUR: NW [233]

— venetum H.H.Blom - RUS-EUR: NW [97, 233], RUS-AS: E-SIB [163], ARC-FE [99]

Schistostega D.Mohr [Schistostegaceae]

— pennata (Hedw.) F.Weber \& D.Mohr - LTV [244], LAT [3], EST [237], BEL [355], UKR: C [66], M 
[66], RUS-EUR: NW [207, 212, 407], NE [207, 212, 415], C [207, 212], N-UR [150, 207, 212], S-UR [150, 207, 212], RUS-AS: W-SIB [265], S-SIB [69, 70, 212], E-SIB [74, 212, 247], YAK [239], N-FE $[94,110,140]$, S-FE [76, 77, 114, 212]

Schwetschkeopsis Broth. [*Hypnaceae]

- fabronia (Schwägr.) Broth. - RUS-AS: S-FE [76, 212]

Sciuro-hypnum (Hampe) Hampe [Brachytheciaceae]

- altaicum (Ignatov) Ignatov, comb. nov. - Eurhynchium altaicum Ignatov, Arctoa 7: 141. 1998. - RUSAS: S-SIB [198] - \{169\}

- brotheri (Paris) Ignatov \& Huttunen - RUS-AS: S-FE [109, 114]

- flotowianum (Sendtn.) Ignatov \& Huttunen (Eurhynchium flotowianum (Sendtn.) Kartt. Cirriphyllum reichenbachianum (Huebener) Wijk \& Margad.) - UKR: C [66], K [66, 328], RUS-EUR: KLN [309], CAUC [208, 212, 232, 248], GEO [117], ARM [297], AZE [277]

- glaciale (Bruch et al.) Ignatov \& Huttunen (Brachythecium glaciale Bruch et al.) - RUS-EUR: ARC [47, 127, 150], NW [87, 364], NE [415], N-UR [150, 207], CAUC [55, 212, 231], GEO [117], TAD [296], KYR [296], RUS-AS: ARC-ESIB [212, 263], S-SIB [70, 198, 212], N-FE [140]

- glaciale var. dovrense (Limpr.) Ochyra (Brachythecium dovrense (Limpr.) J.J.Amann, B. glaciale var. dovrense Limpr.) - RUS-EUR: NW [364], CAUC [231], RUS-AS: S-SIB [198, 212], ARC-FE [40], N-FE [140]

- latifolium (Kindb.) Ignatov \& Huttunen (Brachythecium latifolium Kindb.) - RUS-EUR: ARC [47, 150], NW [212, 273, 364], NE [212, 415], N-UR [150], S-UR [150], RUS-AS: W-SIB [265], S-SIB [70, 198, 212], E-SIB [275], ARC-YAK [47, 239], YAK [239], ARC-FE [40], N-FE [40, 140], S-FE $[212,221]-\{170\}$

- oedipodium (Mitt.) Ignatov \& Huttunen (Brachythecium curtum (Lindb.) Limpr., B. oedipodium (Mitt.) A.Jaeger, B. starkei var. curtum (Lindb.) Warnst.) - LTV [244], LAT [3], EST [237], BEL [355], UKR: C [66], M [66], RUS-EUR: ARC [47, 127, 150], NW [207, 212, 364, 407], NE [207, 212, 415], KLN [309], C [207, 212], N-UR [150, 207, 212], S-UR [150, 207, 212], SE [207, 212], RUS-AS: ARC-WSIB [136], W-SIB [147, 212], S-SIB [70, 198, 212, 265], E-SIB [275], YAK [239], ARC-FE [40], N-FE [40, 140], S-FE [114, 212, 221]

- ornellanum (Molendo) Ignatov \& Huttunen (Brachythecium ornellanum (Molendo) Venturi \& Bott., Scleropodium apiculigerum (Lindb. \& Arnell) J.-P.Frahm, S. ornellanum (Molendo) Lorentz) - RUSEUR: ARC [47, 127, 150], NW [273, 364], NE [212, 415], N-UR [150, 207, 212], S-UR [150, 207], CAUC [231], GEO [15], KAZ [296, 356], KYR [296, 350, 351], RUS-AS: ARC-ESIB [212], W-SIB [147, 212], S-SIB [70, 198, 212], E-SIB [212, 275], ARC-YAK [47, 239], ARC-FE [40], N-FE [140]

- plumosum (Hedw.) Ignatov \& Huttunen (Brachythecium plumosum (Hedw.) Bruch et al.) - LTV [244], LAT [5], EST [237], BEL [355], UKR: C [66], M [66], RUS-EUR: NW [207, 212, 364, 407], NE [53, 207, 212, 415], KLN [309], C [207, 212], N-UR [150, 207, 212], S-UR [150, 207, 212], SE [207], CAUC [212, 231], GEO [117], ARM [297], AZE [277], TAD [296], KYR [296], RUS-AS: S-SIB $[69,198,212]$, E-SIB [212, 275], ARC-YAK [239], YAK [239], ARC-FE [40, 212], N-FE [140], S-FE $[76,77,109,212]$

- populeum (Hedw.) Ignatov \& Huttunen (Brachythecium populeum (Hedw.) Bruch et al.) - LTV [244], LAT [3], EST [237], BEL [355], UKR: C [66], M [66], K [66, 328], RUS-EUR: ARC [47, 150], NW [207, 273, 212, 364, 407], NE [207, 212, 415], KLN [309], C [207, 212], N-UR [150, 207, 212], S-UR [150, 207, 212], SE [207], CAUC [55, 208, 212, 231, 248], GEO [117], ARM [297], AZE [277], KAZ [296, 356], TAD [296], KYR [296], RUS-AS: W-SIB [265], S-SIB [69, 70, 198, 212], E-SIB [212, 275], YAK [239], ARC-FE [40], N-FE [140], S-FE [76, 77, 109, 212]

- reflexum (Starke) Ignatov \& Huttunen (Brachythecium reflexum (Starke) Bruch et al.) - LTV [244], LAT [3], EST [237], BEL [355], UKR: C [66], RUS-EUR: ARC [47, 127, 150], NW [207, 212, 364, 407], NE [207, 212, 415], KLN [309], C [207, 212], N-UR [150, 207, 212], S-UR [150, 207, 212], SE [207, 212], CAUC [55, 231], GEO [117], ARM [297], AZE [277], KAZ [156, 159, 296, 356], TAD [294, 296], KYR [296], RUS-AS: ARC-WSIB [136], W-SIB [212, 265], S-SIB [69, 70, 198, 212], ESIB [212, 275], ARC-YAK [47, 239], YAK [212, 239], ARC-FE [40], N-FE [94, 140], S-FE [76, 77, $109,212]$ 
- starkei (Brid.) Ignatov \& Huttunen (Brachythecium starkei (Brid.) Bruch et al.) - LTV [244], LAT [3], EST [237], BEL [355], UKR: C [66], M [66], RUS-EUR: ARC [47, 150], NW [207, 212, 364, 407], NE [207, 212, 415], KLN [309], C [207], N-UR [150, 207, 212], S-UR [150, 207, 212], CAUC [55, 212, 231, 248], GEO [117], ARM [297], AZE [277], RUS-AS: ARC-WSIB [136], W-SIB [212, 265], S-SIB [69, 70, 198, 212, 265], E-SIB [212, 275], ARC-FE [40], N-FE [40, 140], S-FE [76, 77, 109]

- uncinifolium (Broth. \& Paris) Ochyra \& Żarnowiec (Brachythecium uncinifolium Broth.) - RUS-AS: N-FE [140, 143], S-FE [67, 112, 114]

Scleropodiopsis see Rhynchostegium arcticum

Scleropodium see Pseudoscleropodium purum, Sciuro-hypnum ornellanum

Scopelophila (Mitt.) Lindb. [Pottiaceae]

- ligulata (Spruce) Spruce (Merceya ligulata (Spruce) Schimp., M. ligulata var. acutiuscula (Lindb. ex Broth.) P.C.Chen, M. acutiuscula Lindb. ex Broth.) - GEO [117], RUS-AS: S-FE [76]

Scorpidium (Schimp.) Limpr. [Scorpidiaceae]

- cossonii (Schimp.) Hedenäs (Limprichtia cossonii (Schimp.) L.E.Anderson , L. intermedia (Lindb.) Loeske, Drepanocladus cossonii (Schimp.) Loeske, D. intermedius (Lindb.) Warnst.) - LTV [244], LAT [3], EST [237], BEL [355], UKR: M [66], RUS-EUR: ARC [47, 150], NW [207, 212, 364, 407], NE [207, 212], KLN [309], C [207], N-UR [90, 150, 207], S-UR [150, 207], CAUC [231, 248], GEO [117], ARM [297], KAZ [158], KYR [296], RUS-AS: ARC-WSIB [136], ARC-ESIB [164, 212, 275], W-SIB [265], S-SIB [69, 70, 212], E-SIB [69, 212, 275], ARC-YAK [47, 212, 239], YAK [212, 239], ARC-FE [40, 212], N-FE [94, 140]

- revolvens (Sw. ex anon.) Rubers (Limprichtia revolvens (Sw. ex anon.) Loeske, Drepanocladus revolvens (Sw. ex anon.) Warnst.) - LTV [244], LAT [3], EST [237], BEL [355], UKR: C [66], M [66], RUS-EUR: ARC [47, 127, 150], NW [207, 212, 364, 407], NE [207, 212, 415], KLN [309], N-UR [150, 212], S-UR [150], CAUC [212, 231, 248], GEO [117], ARM [297], KAZ [296, 356], TAD [267, 294, 296], KYR [351], RUS-AS: ARC-WSIB [136], ARC-ESIB [164, 212, 275], W-SIB [147, 212], S-SIB [69, 70, 212], E-SIB [212, 275], ARC-YAK [47, 212, 239], YAK [212, 239], ARC-FE [40, 212], N-FE [94, 140], S-FE [67, 212]

- scorpioides (Hedw.) Limpr. - LTV [244], LAT [3], EST [237], BEL [355], UKR: M [66], RUSEUR: ARC [47, 150], NW [207, 212, 364, 407], NE [207, 212, 415], KLN [309], C [207, 345], N-UR [150, 212], S-UR [150, 207], CAUC [212, 248], RUS-AS: ARC-WSIB [136], ARC-ESIB [164, 212], W-SIB [265], S-SIB [69, 70, 212], E-SIB [69, 212], ARC-YAK [47, 212, 239], YAK [212, 239], ARCFE [40, 212], N-FE [94, 140], S-FE [67]

Scorpiurium Schimp. [Brachytheciaceae]

- circinatum (Brid.) M.Fleisch. \& Loeske - UKR: K [66, 328], RUS-EUR: CAUC [208, 212, 232], GEO [205a], AZE [277]

Scouleria Hook. [Scouleriaceae]

— aquatica Hook. (S. rschewinii Lindb. \& Arnell) - RUS-AS: ARC-ESIB [164, 212, 275], S-SIB [69, 73], E-SIB [212, 260, 275], ARC-YAK [47, 239], YAK [212, 239], N-FE [40, 94], S-FE [212, 221] - \{171\}

- aquatica var. pulcherrima (Broth.) Kurbatova-RUS-AS: ARC-FE [260], ARK-YAK [36], YAK [239, 260], ARC-FE [40, 260], N-FE [40, 260], S-FE [260] - \{171\}

Seligeria Bruch et al. [Seligeriaceae]

- acutifolia Lindb. - GEO [102]

- brevifolia (Lindb.) Lindb. - UKR [401], RUS-EUR: NW [407], N-UR [90, 207, 212], S-UR [91], RUS-AS: S-SIB [69, 212, 275], E-SIB [68, 212]

- calcarea (Hedw.) Bruch et al. - EST [237], UKR: C [66], M [66], RUS-EUR: C [207, 212, 345], SE [207]

- campylopoda Kindb. - LAT [3], EST [237], UKR: C [66], RUS-EUR: NW [207, 281], NE [207, 212, 415], C [207, 212, 345], N-UR [90, 207, 302], S-UR [177], RUS-AS: W-SIB [307], S-SIB [212], ESIB [162], ARC-YAK [239], YAK [239]

- diversifolia Lindb. - RUS-EUR: NW [207, 364, 407], RUS-AS: S-SIB [69, 70, 212] - $\{172\}$

— donniana (Sm.) Müll.Hal. - EST [237], UKR: C [66], M [66], RUS-EUR: NW [407], NE [415], C [207], S-UR [88, 207, 302], GEO [13], TUR [267, 296], KYR [267, 296], RUS-AS: S-SIB [69, 70, $275]-\{173\}$ 
— galinae Mogensen \& I.Goldberg - RUS-EUR: C [313], N-UR [90, 207, 212, 302], S-UR [91] $\{173\}$

- oelandica C.E.O.Jensen \& Medelius - RUS-AS: ARC-FE [40]

— patula (Lindb.) I.Hagen (S. alpestris T.Schauer, S. patula var. alpestris (T.Schauer) Gos \& Ochyra, $S$. tristichoides var. patula (Lindb.) Broth.) - EST [237]

- polaris Berggr. - RUS-EUR: ARC [47], RUS-AS: ARC-ESIB [39, 212, 263], ?S-SIB [69, 70], E-SIB [163], ARC-YAK [47, 239], ARC-FE [40, 212] - \{174\}

- pusilla (Hedw.) Bruch et al. - LAT [3], EST [237], UKR: C [66], M [66], K [66, 328], RUS-EUR: C [207, 212, 345], NE [415], S-UR [207, 212, 302], SE [207, 366], CAUC [55, 212, 248], AZE [277], TUR [267, 296], TAD [295], KYR [267, 296], RUS-AS: S-SIB [212, 336]

- recurvata (Hedw.) Bruch et al. - LAT [3], EST [237], UKR: C [66], M [66], K [66, 328], RUS-EUR: NW [407], CAUC [232], GEO [102, 117], AZE [277], RUS-AS: S-FE [76]

- subimmersa Lindb. - RUS-EUR: NW [407]

- trifaria (Brid.) Lindb. - RUS-EUR: NE [207, 415], CAUC [55, 212], GEO [102], RUS-AS: ?S-SIB [70], ?E-SIB [275] - $\{175\}$

— tristichoides Kindb. - RUS-EUR: NW [364, 407], N-UR [90, 207, 212, 302], RUS-AS: ARC-ESIB [164, 212], S-SIB [212], E-SIB [163], YAK [239]

Semibarbula see Tortula

Serpoleskea (Limpr.) Loeske [Amblystegiaceae]

- confervoides (Brid.) Loeske (Amblystegium confervoides (Brid.) Bruch et al., Amblystegiella confervoides (Brid.) Loeske, Platydictya confervoides (Brid.) H.A.Crum) - LAT [3], EST [237], BEL [355], UKR: C [66], M [66], RUS-EUR: NW [407], NE [207, 416], C [207, 345], S-UR [91, 150, 207, 212], CAUC [248], GEO [117], ARM [297], AZE [277], KAZ [156, 296], TAD [296], KYR [296], RUSAS: S-SIB [212, 336]

- subtilis (Hedw.) Loeske (Amblystegium subtile (Hedw.) Bruch et al., Amblystegiella subtilis (Hedw.) Loeske, Platydictya subtilis (Hedw.) H.A.Crum) - LTV [244], LAT [3], EST [237], BEL [355], UKR: C [66], M [66], K [66, 328], MLD [367], RUS-EUR: ARC [47, 127, 150], NW [207, 407], NE [207, 212, 415], KLN [309], C [207, 212], N-UR [90, 150, 207], S-UR [150, 207, 212], SE [207, 212], CAUC [55, 212, 231, 248), GEO [117], ARM [297], AZE [277], TAD [296], KYR [267, 296], RUSAS: ARC-WSIB [136], W-SIB [265], S-SIB [70, 212], YAK [?239], S-FE [76, 212]

Sphagnum L. [Sphagnaceae]

— affine Renauld \& Cardot (S. affine var. flagellare (Schlieph. ex Röll) L.Söderstr. \& Hedenäs, S. imbricatum subsp. affine (Renauld \& Cardot) Flatberg) - RUS-EUR: NW [290], ARC [?47], RUS-AS: ARC-WSIB [?47], ARC-YAK [?239]

- angustifolium (C.E.O.Jensen ex Russow) C.E.O.Jensen - LTV [244], LAT [3], EST [237], BEL [333], UKR: C [412], M [412], RUS-EUR: ARC [47, 127, 150], NW [207, 212, 364, 407], NE [207, 212, 415], KLN [310], C [207, 212], N-UR [150, 207], S-UR [150, 207, 212], SE [207, 212], CAUC [55, 212], GEO [117], KAZ [356], RUS-AS: ARC-WSIB [136], ARC-ESIB [164], W-SIB [212, 265], S-SIB [70, 212], E-SIB [74, 212], ARC-YAK [47, 239], YAK [212, 239], ARC-FE [40], N-FE [94, 140], S-FE [76, 109, 212]

— annulatum H.Lindb. ex Warnst. - RUS-EUR: NW [212, 262, 282], ?NE [212, 417], ?RUS-AS: E-SIB $[212,308]-\{176\}$

- aongstroemii Hartm. - EST [237], RUS-EUR: ARC [47, 127, 150], NW [207, 212, 364, 262, 407], NE [207, 212, 415], C [207], N-UR [150, 207, 212], S-UR [150, 212], RUS-AS: ARC-WSIB [136], ARC-ESIB [164, 212], W-SIB [212, 265], S-SIB [70, 212], E-SIB [74, 212], ARC-YAK [47, 212, 239], YAK [212, 239], ARC-FE [40, 212], N-FE [94], S-FE [212, 221]

— arcticum Flatberg \& Frisvoll - RUS-AS: ARC-YAK [284], ARC-FE [40, 212]

— auriculatum Schimp. (S. denticulatum Brid.) - LTV [244], LAT [3], EST [237], BEL [333], UKR: M [412], RUS-EUR: KLN [309], NW [212, 364, 407], C [207, 212], CAUC [231], GEO [117], RUSAS: ARC-ESIB [164], S-FE [30]

— austinii Sull. (S. imbricatum subsp. austinii (Sull.) Flatberg) - LAT [4], EST [237], GEO [168] — $\{177\}$ 
- balticum (Russow) C.E.O.Jensen - LTV [244], LAT [3], EST [237], BEL [333], UKR: C [413], RUS-EUR: ARC [47, 150], NW [207, 212, 364, 407], NE [207, 212, 415], KLN [310], C [207, 212, 345], N-UR [150, 207, 212], S-UR [150, 207, 212], SE [207], CAUC [55, 212], RUS-AS: ARC-WSIB [136], ARC-ESIB [164, 212], W-SIB [212, 265], S-SIB [70, 212], E-SIB [74, 212], ARC-YAK [47, 212, 239], YAK [212, 239], ARC-FE [40, 212], N-FE [94, 140], S-FE [109, 212, 221]

- capillifolium (Ehrh.) Hedw. (S. nemoreum Scop., S. subtile (Russow) Warnst.) - LTV [244], LAT [3], EST [237], BEL [333], UKR: C [412], M [412], RUS-EUR: ARC [47, 127, 150], NW [207, 212, 364, 407], NE [207, 212, 415], KLN [310], C [207, 212], N-UR [150, 207, 212], S-UR [150, 207, 212], SE [207], CAUC [55, 212, 231, 248], GEO [117], KAZ [26, 296], RUS-AS: ARC-WSIB [136], ARCESIB [164, 212], W-SIB [212, 265], S-SIB [70, 212], E-SIB [212, 247], ARC-YAK [47, 212, 239], YAK [212, 239], ARC-FE [40], N-FE [94, 140], S-FE [76, 77, 109, 212]

- centrale C.E.O.Jensen - LTV [244], LAT [3], EST [237], BEL [333], UKR: C [412], M [412], RUSEUR: ARC [47, 150], NW [207, 212, 364, 407], NE [207, 212, 415], KLN [310], C [207, 212], N-UR [150, 207, 212], S-UR [150, 207, 212], SE [207], CAUC [55, 212, 231, 248], GEO [117], AZE [277], KAZ [26, 296], RUS-AS: ARC-WSIB [136], W-SIB [212, 265], S-SIB [70, 212], E-SIB [74, 212, 247], YAK [239], ARC-FE [40], N-FE [140], S-FE [76, 109, 212]

- compactum Lam. \& DC. - LTV [244], LAT [3], EST [237], BEL [333], UKR: C [412], M [412], RUS-EUR: ARC [47, 150], NW [207, 212, 364, 407], NE [207, 212, 415], KLN [310], C [207, 212], N-UR [150, 207, 212], S-UR [150, 207], SE [207], CAUC [55, 212], GEO [117], RUS-AS: ARCWSIB [136], ARC-ESIB [212], W-SIB [212, 265], S-SIB [70, 212], E-SIB [74, 212], ARC-YAK [47, 212, 239], YAK [212, 239], ARC-FE [40, 212], N-FE [94, 140], S-FE [212, 221]

- contortum Schultz - LTV [244], LAT [3], EST [237], BEL [333], UKR: C [412], M [412], RUS-EUR: ARC [47, 150], NW [207, 212, 262, 364, 407], NE [207, 212, 415], KLN [310], C [207, 212, 345], NUR [150, 212, 207], S-UR [150, 207], SE [207], CAUC [55, 212, 231], GEO [117], KAZ [26, 296], RUS-AS: ARC-WSIB [136], ARC-ESIB [164, 212], W-SIB [265], S-SIB [212, 336], E-SIB [212, 247], ARC-YAK [47, 212, 239], YAK [212, 239], ARC-FE [40, 212], N-FE [94, 140], S-FE [76, 212]

- cuspidatum Ehrh. ex Hoffm. - LTV [244], LAT [3], EST [237], BEL [333], UKR: M [412], RUSEUR: ARC [47, 150], NW [207, 212, 364, 407], NE [207, 212, 415], KLN [310], C [207, 212, 345], N-UR [150, 207], S-UR [150], SE [207], CAUC [55, 212], GEO [117], RUS-AS: W-SIB [212, 265], S-SIB [70], YAK [212, 239], N-FE [94, 109, 140]

— ?elenkinii B.S.Semenov - RUS-AS: S-SIB [365] - $\{178\}$

- fallax (H.Klinggr.) H.Klinggr. - LTV [244], LAT [3], EST [237], BEL [333], UKR: C [412], M [412], RUS-EUR: ARC [47, 150], NW [207, 212, 364, 407], NE [207, 212, 415], KLN [310], C [207, 212], N-UR [150, 207, 212], S-UR [150, 207, 212], SE [207], CAUC [55, 212], GEO [117], RUS-AS: ARC-WSIB [47], W-SIB [212, 265], S-SIB [70, 212], E-SIB [74, 212, 247], ARC-YAK [47, 239], YAK [212, 239], ARC-FE [40], N-FE [140], S-FE [77, 109, 212]

- fimbriatum Wilson - LTV [244], LAT [3], EST [237], BEL [333], UKR: C [412], M [412], RUSEUR: ARC [47, 150], NW [207, 212, 364, 407], NE [207, 212, 415], KLN [310], C [207, 212], N-UR [150, 212, 207], S-UR [150, 207], SE [207, 212], GEO [117], ARM [297], RUS-AS: ARC-WSIB [136], ARC-ESIB [164, 212], W-SIB [212, 265], S-SIB [212], E-SIB [126, 212], ARC-YAK [47, 212, 239], YAK [239], ARC-FE [40, 212], N-FE [94, 140], S-FE [76, 77, 109, 212]

- flexuosum Dozy \& Molk. - LTV [244], LAT [3], EST [237], BEL [333], UKR: C [412], M [412], RUS-EUR: ARC [47, 150], NW [207, 212, 364, 407], NE [207, 212, 415], KLN [310], C [207, 212], N-UR [150, 207, 212], S-UR [150, 207, 212], SE [207], CAUC [55, 212], GEO [117], KAZ [26, 296, 356], RUS-AS: ARC-WSIB [136], ARC-ESIB [93], W-SIB [212, 265], S-SIB [70, 212], ARC-YAK [47, 212, 239], YAK [239], ARC-FE [40], N-FE [94, 140], S-FE [76, 212, 221]

- fuscum (Schimp.) H.Klinggr. - LTV [244], LAT [3], EST [237], BEL [333], UKR: C [412], M [412], RUS-EUR: ARC [47, 150], NW [207, 212, 364, 407], NE [207, 212, 415], KLN [310], C [207, 212, 345], N-UR [150, 207, 212], S-UR [150, 207, 212], SE [207], GEO [117], ARM [297], KAZ [26, 295, 296, 356], TAD [26, 295], RUS-AS: ARC-WSIB [136], ARC-ESIB [212], W-SIB [212, 265], SSIB [70, 212], E-SIB [74, 212, 247], ARC-YAK [47], YAK [212, 239], ARC-FE [40], N-FE [94, 140], S-FE [76, 109, 212] 
- girgensohnii Russow - LTV [244], LAT [3], EST [237], BEL [333], UKR: C [412], M [412], RUSEUR: ARC [47, 127, 150], NW [207, 212, 364, 407], NE [207, 212, 415], KLN [310], C [207, 212], N-UR [150, 207, 212], S-UR [150, 207, 212], SE [207, 212], CAUC [55, 212, 231, 248], GEO [117], ARM [297], KAZ [26, 296, 356], RUS-AS: ARC-WSIB [136], ARC-ESIB [164, 212], W-SIB [212, 265], S-SIB [70, 212], E-SIB [74, 212, 247], ARC-YAK [47, 212, 239], YAK [212, 239], ARC-FE [40, 212], N-FE [94, 140], S-FE [76, 77, 109, 212]

— ?gordjaginii B.S.Semenov - RUS-AS: S-SIB [365] - $\{178\}$

— imbricatum Hornsch. ex Russow s. str. - RUS-AS: YAK [168, 239], N-FE [168], S-FE [30, 76, 168] $-\{179\}$

- inexpectatum Flatberg - RUS-AS: ARC-FE [170]

- inundatum Russow (S. denticulatum var. inundatum (Russow) Kartt., S. subsecundum subsp. inundatum (Russow) Meyl., S. subsecundum var. inundatum (Russow) C.E.O.Jensen) - LTV [244], LAT [1], EST [237], BEL [333], UKR: C [406], M [412], RUS-EUR: ARC [47, 150], NW [207, 407], NE [207, 415], KLN [310], C [207, 212], CAUC [55, 212], GEO [117], RUS-AS: S-FE [109]

- jensenii H. Lindb. - LTV [244], LAT [3], EST [237], BEL [333], RUS-EUR: ARC [47, 150], NW [207, 212, 364, 407], NE [207, 212, 415], C [207, 212], N-UR [150, 207, 212], S-UR [150, 207, 212], RUS-AS: ARC-WSIB [136], ARC-ESIB [212], W-SIB [212, 265], S-SIB [212], E-SIB [212, 247], YAK [239], N-FE [140], S-FE [109, 212, 221] - $\{176\}$

- ?krylovii B.S.Semenov - RUS-AS: S-SIB [365] $-\{178\}$

- lenense H.Lindb. ex L.I.Savicz - RUS-EUR: ARC [47, 127, 150, 415], N-UR [150, 415], RUS-AS: ARC-WSIB [136], ARC-ESIB [164, 212], W-SIB [265], E-SIB [74, 212], ARC-YAK [47, 212, 239], YAK [212, 239], ARC-FE [40, 212], N-FE [94], S-FE [109, 212, 221]

- lindbergii Schimp. - LAT [3], EST [237], BEL [333], RUS-EUR: ARC [47, 127, 150], NW [207, 212, 364, 407], NE [207, 212, 415], C [207, 212], N-UR [150, 207, 212], S-UR [150, 207, 212], RUSAS: ARC-WSIB [136], ARC-ESIB [?47], W-SIB [212, 265], S-SIB [197, 212], E-SIB [74, 212], ARC-YAK [47, 239], YAK [239], ARC-FE [40], N-FE [94, 140], S-FE [109, 212, 221]

- magellanicum Brid. - LTV [244], LAT [3], EST [237], BEL [333], UKR: C [412], M [412], RUSEUR: ARC [47, 150], NW [207, 212, 364, 407], NE [207, 212, 415], KLN [310], C [207, 212], NUR [150, 207, 212], S-UR [150, 207, 212], SE [207, 212], CAUC [55, 212, 231], GEO [117], KAZ [26, 296], RUS-AS: ARC-WSIB [136], ARC-ESIB [212], W-SIB [212, 265], S-SIB [70, 212], ESIB [74, 212, 247], ARC-YAK [47, 239], YAK [212, 239], ARC-FE [40], N-FE [94, 140], S-FE [76, $77,109,212]$

- majus (Russow) C.E.O.Jensen - LTV [244], LAT [3], EST [237], BEL [333], UKR: M [412], RUSEUR: ARC [47, 150], NW [207, 212, 364, 407], NE [207, 212, 415], KLN [310], C [207, 212], N-UR [150, 207, 212], S-UR [150, 207], SE [207], RUS-AS: W-SIB [212, 265], S-SIB [212, 336], ARCYAK [47, 239], YAK [239], ARC-FE [40], N-FE [140]

- molle Sull. - LTV [244], LAT [3], EST [237], BEL [333], UKR: M [412], RUS-EUR: NW [280, 407], KLN [310]

- obtusum Warnst. - LTV [244], LAT [3], EST [237], BEL [333], UKR: C [412], M [412], RUS-EUR: ARC [47, 150], NW [207, 212, 407], NE [207, 212, 415], KLN [310], C [207, 212], N-UR [90, 150, 207], S-UR [150, 207], SE [207], GEO [117], RUS-AS: ARC-WSIB [136], ARC-ESIB [164, 212], W-SIB [212, 265], S-SIB [212, 336], E-SIB [74, 212, 247], ARC-YAK [47, 212, 239], YAK [212, 239], ARC-FE [40], N-FE [94, 140], S-FE [76, 109, 212]

- orientale L.I.Savicz - RUS-AS: ARC-WSIB [136], ARC-ESIB [164, 212], E-SIB [126, 212], ARCYAK [47, 212, 239], YAK [212, 239], ARC-FE [40, 212], N-FE [40, 94], S-FE [76, 212]

- palustre L. - LTV [244], LAT [3], EST [237], BEL [333], UKR: C [412], M [412], RUS-EUR: NW [207, 407], NE [207, 212, 415], KLN [310], C [207, 212, 345], N-UR [207], S-UR [150, 207, 212], SE [207], CAUC [212, 248], GEO [117], KAZ [26, 296], RUS-AS: W-SIB [265], S-SIB [70, 212], E-SIB [212, 247], S-FE [76, 77, 109, 212]

- papillosum Lindb. - LTV [244], LAT [3], EST [237], BEL [333], UKR: C [412], M [412], RUSEUR: NW [207, 212, 364, 407], NE [207, 212, 415], KLN [310], C [207, 212, 345], N-UR [150, 207, 212], S-UR [150, 207, 212], SE [207], GEO [117], RUS-AS: W-SIB [212, 265], S-SIB [212, 336], E- 
SIB [212, 308], ARC-FE [40], N-FE [140], S-FE [109]

- perfoliatum L.I.Savicz - RUS-AS: ARC-WSIB [136], WSIB [428], ARC-YAK [47, 239], ARC-FE [40], N-FE [94, 95]

- platyphyllum (Lindb. ex Braithw.) Warnst. - LTV [244], LAT [3], EST [237], BEL [333], UKR: C [412], M [412], RUS-EUR: ARC [47, 150], NW [207, 212, 364, 407], NE [53, 207, 212, 415], KLN [310], C [207, 212, 345], N-UR [90, 150, 207], S-UR [150, 207], SE [207], CAUC [55, 212, 231], GEO [117], KAZ [26, 296], RUS-AS: ARC-WSIB [136], ARC-ESIB [164, 212], W-SIB [212, 265], S-SIB [197, 212], E-SIB [74, 212, 247], ARC-YAK [47, 212, 239], YAK [239], ARC-FE [40, 212], NFE [140], S-FE [212, 109]

- pulchrum (Lindb. ex Braithw.) Warnst. - LTV [244], LAT [3], BEL [333], RUS-EUR: NW [207, 212, 407], NE [207, 415], KLN [310], C [207], N-UR [150, 415], RUS-AS: S-SIB [212, 336], ARCYAK [47, 239], N-FE [140], S-FE [77]

- quinquefarium (Lindb. ex Braithw.) Warnst. - LTV [244], LAT [3], EST [237], BEL [333], UKR: C [412], RUS-EUR: NW [212, 364, 407], NE [288, 207, 212, 415], KLN [310], C [207, 212], N-UR [150, 207], S-UR [150], CAUC [212, 248], GEO [117], RUS-AS: W-SIB [265], S-SIB [70, 212], ESIB [197, 212], S-FE [67]

— riparium Ångstr. - LTV [244], LAT [3], EST [237], BEL [333], UKR: C [412], M [412], RUS-EUR: ARC [47, 127, 150], NW [207, 212, 364, 407], NE [207, 212, 415], KLN [310], C [207, 212], N-UR [150, 207, 212], S-UR [150, 207, 212], SE [207], ARM [297], RUS-AS: ARC-WSIB [136], ARCESIB [?47, 93], W-SIB [212, 265], S-SIB [70, 212], E-SIB [74, 212, 247], ARC-YAK [47, 239], YAK [239], ARC-FE [40], N-FE [94, 140], S-FE [109, 212, 221]

- rubellum Wilson - LTV [244], LAT [3], EST [237], BEL [333], UKR: C [412], M [412], RUS-EUR: ARC [47, 127, 150], NW [207, 212, 407], NE [207, 212, 415], KLN [310], C [207, 212], N-UR [150, 207, 212], S-UR [150, 207], SE [207], CAUC [55, 212], GEO [117], RUS-AS: ARC-WSIB [136], ARC-ESIB [96, 212], W-SIB [265], S-SIB [70, 212], E-SIB [74, 212, 247], ARC-YAK [47, 212, 239], YAK [212, 239], ARC-FE [40, 212], N-FE [140], S-FE [212, 109]

- russowii Warnst. - LTV [244], LAT [3], EST [237], BEL [333], UKR: C [412], M [412], RUSEUR: ARC [47, 127, 150], NW [207, 212, 364, 407], NE [207, 212, 415], KLN [310], C [207, 212], N-UR [150, 207, 212], S-UR [150, 207, 212], SE [207], CAUC [55, 212, 248], GEO [117], KAZ [26, 296], RUS-AS: ARC-WSIB [136], ARC-ESIB [93, 164], W-SIB [212, 265], S-SIB [70, 212], E-SIB [74, 212, 247], ARC-YAK [47, 212, 239], YAK [212, 239], ARC-FE [40, 212], N-FE [94, 140], S-FE [109, 212, 221]

- squarrosum Crome - LTV [244], LAT [3], EST [237], BEL [333], UKR: C [412], M [412], RUSEUR: ARC [47, 127, 150], NW [207, 212, 364, 407], NE [207, 212, 415], KLN [310], C [207, 212], N-UR [150, 207, 212], S-UR [150, 207, 212], SE [207, 212], CAUC [55, 212, 231, 248], GEO [117], ARM [297], KAZ [26, 296, 356], RUS-AS: ARC-WSIB [136], ARC-ESIB [164, 212], W-SIB [212, 265], S-SIB [70, 212], E-SIB [74, 212, 247], ARC-YAK [47, 212, 239], YAK [212, 239], ARC-FE [40, 212], N-FE [94, 140], S-FE [76, 77, 109, 212]

- steerei R.E.Andrus (S. imbricatum var. arcticum Flatberg) - RUS-AS: ARC-YAK [?239], YAK [239], ARC-FE [168]

— subfulvum Sjörs - LAT [3], EST [237], RUS-EUR: NW [212, 273, 364], NE [207], N-UR [207], RUS-AS: ARC-YAK [239], ARC-FE [40], N-FE [40]

- subnitens Russow \& Warnst. - LTV [244], LAT [3], EST [237], BEL [333], UKR: M [412], RUSEUR: NW [212, 273, 364, 407], NE [207], KLN [310], S-UR [150, 207], KAZ [26, 296], RUS-AS: W-SIB [265], S-SIB [212, 336]

- subsecundum Nees - LTV [244], LAT [3], EST [237], BEL [333], UKR: C [412], M [412], RUSEUR: ARC [47, 150], NW [207, 212, 364, 407], NE [207, 212, 415], KLN [310], C [207, 212], N-UR [150, 207, 212], S-UR [150, 207], SE [207], CAUC [55, 212, 231, 248], GEO [117], AZE [277], KAZ [26, 296, 356], RUS-AS: ARC-WSIB [136], ARC-ESIB [164, 212], W-SIB [212, 265], S-SIB [212, 336], E-SIB [163], ARC-YAK [47, 212, 239], YAK [212, 239], ARC-FE [40, 212], N-FE [140], S-FE [76, 109, 212]

— tenellum (Brid.) Pers. ex Brid. - LTV [244], LAT [3], EST [237], BEL [333], UKR: M [412], RUS- 
EUR: NW [207, 212, 364, 407], NE [207], KLN [310], C [207], CAUC [55, 212], RUS-AS: S-SIB [212, 336], E-SIB [212, 308], N-FE [140]

- teres (Schimp.) Ångstr. - LTV [244], LAT [3], EST [237], BEL [333], UKR: C [412], M [412], RUSEUR: ARC [47, 127, 150], NW [207, 212, 364, 407], NE [207, 212, 415], KLN [310], C [207, 212], N-UR [150, 207, 212], S-UR [150, 207], SE [207, 212], CAUC [55, 212, 231, 248], GEO [117], KAZ [26, 295, 296, 356], TAD [26, 295], RUS-AS: ARC-WSIB [136], ARC-ESIB [164, 212, 275], W-SIB $[212,265]$, S-SIB [70, 212], E-SIB [74, 212, 247], ARC-YAK [47, 212, 239], YAK [212, 239], ARCFE [40, 212], N-FE [94, 140], S-FE [212, 77, 109]

- tundrae Flatberg - RUS-AS: ARC-FE [40]

- ?vereschaginii B.S.Semenov - RUS-AS: S-SIB [365] - $\{178\}$

- warnstorfii Russow - LTV [244], LAT [3], EST [237], BEL [333], UKR: M [412], RUS-EUR: ARC $[47,127,150]$, NW [207, 212, 364, 407], NE [207, 212, 415], KLN [310], C [207, 212], N-UR [150, 207, 212], S-UR [150, 207, 212], SE [207, 212], CAUC [55, 212, 231, 248], GEO [117], KAZ [26], RUS-AS: ARC-WSIB [136], ARC-ESIB [164, 212], W-SIB [212, 265], S-SIB [70, 212], E-SIB [74, 212, 247], ARC-YAK [47, 212, 239], YAK [239], ARC-FE [40, 212], N-FE [94, 140], S-FE [109, 212, 221]

- wulfianum Girg. - LTV [244], LAT [3], EST [237], BEL [333], UKR: M [412], RUS-EUR: ARC [47, 150], NW [207, 212, 364, 407], NE [207, 212, 415], C [207, 212], N-UR [150, 207, 212], S-UR [150, 207, 212], SE [207], KAZ [296], RUS-AS: W-SIB [212, 265], S-SIB [70, 212], E-SIB [74, 212, 247], ARC-YAK [239], YAK [212, 239], S-FE [212, 109]

Splachnum Hedw. [Splachnaceae]

- ampullaceum Hedw. - LTV [244], LAT [3], EST [237], BEL [355], UKR: C [66], M [66], RUSEUR: NW [207, 212, 364, 407], NE [207, 415], KLN [309], C [207, 212], N-UR [150, 207], S-UR [150, 207, 212], TAD [296], RUS-AS: W-SIB [265], S-SIB [69, 70, 212], E-SIB [69, 163], YAK [239], N-FE [94]

- luteum Hedw. - RUS-EUR: ARC [47, 150], NW [207, 212, 364, 407], NE [207, 212, 415], C [207, 212], N-UR [150, 207, 212], S-UR [207], RUS-AS: W-SIB [212, 265], S-SIB [69, 70, 212], E-SIB [69, 162, 275], ARC-YAK [47, 239], YAK [239], ARC-FE [40, 212], N-FE [94, 140]

- melanocaulon (Wahlenb.) Schwägr. - RUS-EUR: NW [207, 262], NE [414], RUS-AS: E-SIB [275], N-FE [140, 275]

- pensylvanicum (Brid.) Grout ex H.A.Crum - LAT [3]

- rubrum Hedw. - LAT [3], EST [237], RUS-EUR: ARC [47, 150], NW [207, 212, 364, 407], NE [207, 212, 415], C [207, 212], N-UR [150, 207, 415], RUS-AS: W-SIB [212, 265], S-SIB [69, 212], ESIB [69, 275], ARC-YAK [47, 239], YAK [239], ARC-FE [38, 212], N-FE [94, 140], S-FE [212, 221]

- sphaericum Hedw. (S. ovatum Hedw.) - LAT [3], EST [237], UKR: C [66], RUS-EUR: ARC [47, 127, 150, 415], NW [207, 212, 364, 407], NE [207, 415], N-UR [150], KAZ [296, 356], RUS-AS: ARC-WSIB [136], ARC-ESIB [164, 212, 275], W-SIB [147, 212], S-SIB [69, 70, 212], E-SIB [69, 212, 275], ARC-YAK [47, 239], YAK [239], ARC-FE [40], N-FE [40, 140], S-FE [212, 221]

- vasculosum Hedw. - EST [237], RUS-EUR: ARC [47, 150], NW [87, 212, 364, 407], N-UR [150], KAZ [157], KYR [296], RUS-AS: ARC-WSIB [136], ARC-ESIB [164, 212, 275], W-SIB [212, 265], E-SIB [163], ARC-YAK [47, 239], YAK [239], ARC-FE [40, 212], N-FE [40]

Stegonia Venturi [Pottiaceae]

- latifolia (Schwägr.) Venturi ex Broth. - RUS-EUR: ARC [47, 150, 415], CAUC [55, 212, 231], GEO [18], ARM [297], KAZ [157, 296], TAD [267, 295], KYR [296, 349, 351], RUS-AS: ARC-WSIB [136], ARC-ESIB [164, 212, 275], S-SIB [70, 212], E-SIB [126, 212], ARC-YAK [47, 239], YAK [239], ARC-FE [40, 212], N-FE [40]

- pilifera (Brid.) H.A.Crum \& L.E.Anderson - GEO [102], TAD [295], KYR [296, 349], RUS-AS: ARC-WSIB [136], S-SIB [70, 212], ARC-FE [40, 212], N-FE [40]

Stereodon (Brid.) Mitt. [Pylaisiaceae] $-\{180\}$

- bambergeri (Schimp.) Lindb. (Hypnum bambergeri Schimp.) - UKR: C [66], RUS-EUR: ARC [47, 150, 415], NW [364], NE [207, 212], N-UR [150], KAZ [159, 296, 356], KYR [296], RUS-AS: ARCWSIB [136], ARC-ESIB [164, 212], S-SIB [69, 70, 212], E-SIB [126, 212, 247], ARC-YAK [47, 212, 239], YAK [212, 239], ARC-FE [40, 212], N-FE [94, 140], S-FE [76] 
- callichrous (Brid.) Braithw. (Hypnum callichroum Brid.) - UKR: C [66], RUS-EUR: NW [212, 273, 364, 407], CAUC [212, 231], GEO [117], ARM [297], RUS-AS: S-SIB [70], N-FE [140], S-FE [268] $-\{181\}$

- fauriei (Cardot) Ignatov \& Ignatova - Hypnum fauriei Cardot, Beih. Bot. Centralbl. 17: 41, f. 26.1904. - RUS-AS: S-SIB [200, 212], YAK [239], S-FE [212, 221] - \{182\}

- fertilis (Sendtn.) Lindb. (Hypnum fertile Sendtn.) - LAT [3], BEL [355], UKR: C [66], M [66], RUSEUR: NW [48], C [207], S-UR [150], CAUC [212, 248], GEO [117], AZE [277], TAD [296], KYR [296] $-\{182\}$

- hamulosus (Bruch et al.) Lindb. (Hypnum hamulosum Bruch et al.) - UKR: C [66], RUS-EUR: ARC [150], NW [41, 87, 364, 407], NE [415], N-UR [41, 150, 415], AZE [277], KAZ [159, 296], RUS-AS: ARC-WSIB [?47], ARC-ESIB [41, 164], S-SIB [41, 70, 212], E-SIB [163], ARC-YAK [41, 49, 239], YAK [239], ARC-FE [40, 41], N-FE [140]

- holmenii (Ando) Ignatov \& Ignatova (Hypnum holmenii Ando) - RUS-EUR: ARC [41], NW [41, 207], NE [41, 212], N-UR [41, 90], RUS-AS: ARC-WSIB [41, 136], ARC-ESIB [41, 164], S-SIB [41, 212], E-SIB [41], ARC-YAK [41, 212, 239], YAK [41, 239], ARC-FE [40, 41], N-FE [41, 140], S-FE $[41]-\{183\}$

— pallescens (Hedw.) Mitt. (Hypnum pallescens (Hedw.) P.Beauv.) - LTV [244], LAT [3], EST [237], BEL [355], UKR: C [66], M [66], K [66, 328], MLD [367], RUS-EUR: NW [207, 212, 407], NE [207, 212, 416], KLN [309], C [207, 212], N-UR [150, 207, 212], S-UR [150, 207, 212], SE [207, 212], CAUC [55, 212, 231], GEO [117], ARM [297], AZE [277], KAZ [296], RUS-AS: W-SIB [212, 265], S-SIB [70, 212], E-SIB [212, 275, 388], N-FE [140], S-FE [77, 109, 212] - \{184\}

- plicatulus Lindb. (Hypnum plicatulum (Lindb.) A.Jaeger) - RUS-EUR: ARC [41, 47, 150, 127], NW [41, 212, 273], NE [41, 212], N-UR [41, 150, 207, 212, 415], S-UR [41, 207], RUS-AS: ARC-WSIB [41, 136], ARC-ESIB [41, 212], W-SIB [41, 212, 265], S-SIB [41, 212], E-SIB [41, 212, 275], ARCYAK [41, 47, 212, 239], YAK [41, 212, 239], ARC-FE [40, 41, 212], N-FE [41, 90, 140], S-FE [41, 76, $77,109,212]$

— plumaeformis (Wilson) Mitt. (Hypnum plumaeforme Wilson) - GEO [29], RUS-AS: S-FE [29, 76, 77, 212]

- procerrimus (Molendo) Bauer - RUS-EUR: ARC [47, 150], CAUC [55, 212, 231, 248], GEO [117], ARM [297], KAZ [156, 159], TAD [296], KYR [296], RUS-AS: ARC-WSIB [136], ARCESIB [164, 212], S-SIB [70, 212], E-SIB [163, 388], ARC-YAK [47, 239], YAK [239], ARC-FE $[40,212]-\{185\}$

- revolutus Mitt. (Hypnum revolutum (Mitt.) Lindb.) - UKR: C [66], K [66, 328], RUS-EUR: ARC [47, 150], NW [364], NE [415], N-UR [150], S-UR [150], CAUC [55, 212, 231, 248], GEO [117], ARM [297], AZE [277], KAZ [156, 159, 267, 296, 356], TUR [296], UZB [267], TAD [294], KYR [267, 296, 351], RUS-AS: ARC-WSIB [136], ARC-ESIB [164, 212], S-SIB [70, 212], E-SIB [126, 212], ARC-YAK [47, 212, 239], YAK [239], ARC-FE [40, 212], N-FE [94, 140]

- subimponens (Lesq.) Broth. (Hypnum subimponens Lesq.) - BEL [?355], RUS-EUR: NW [41], NE [41], RUS-AS: ARC-WSIB [41, 136], ARC-ESIB [41, 164, 212], S-SIB [41], E-SIB [41, 212], ARCYAK [41, 239], YAK [14, 17, 212, 239], ARC-FE [40, 41, 212], N-FE [41, 94, 140], S-FE [41, 212]

- subimponens var. ulophyllum (Müll. Hal.) Afonina, comb. nov. - Cupressina ulophylla Müll. Hal., Nuov. Giorn. Bot. Ital. n. ser. 3: 122. 1896. - RUS-AS: YAK [41], ARC-FE [41], S-FE [41]

- tristo-viridis Broth. (Hypnum tristo-viride (Broth.) Paris) - excluded - $\{186\}$

— vaucheri (Lesq.) Lindb. ex Broth. (Hypnum vaucheri Lesq.) - UKR: C [66], M [66], K [66, 328], RUS-EUR: ARC [47, 150], NW [212, 364, 407], NE [212, 415], C [207, 212, 345], N-UR [150, 207, 212], S-UR [150, 207, 212], SE [207], CAUC [55, 212, 231, 248], GEO [117], ARM [297], AZE [277], KAZ [156, 159, 267, 296, 356], TUR [267, 296], TAD [294, 296], KYR [267, 296, 351], RUSAS: ARC-WSIB [136], ARC-ESIB [164, 212], S-SIB [69, 70, 212], E-SIB [212, 275], ARC-YAK [47, 239], YAK [212, 239], ARC-FE [40, 212], N-FE [94, 140], S-FE [76, 212]

Straminergon Hedenäs [Calliergonaceae]

- stramineum (Dicks. ex Brid.) Hedenäs (Calliergon stramineum (Dicks. ex Brid.) Kindb.) - LTV [244], LAT [3], EST [237], BEL [355], UKR: C [66], M [66], RUS-EUR: ARC [47, 127, 150], 
NW [207, 212, 364, 407], NE [207, 212, 415], KLN [309], C [207, 212], N-UR [150, 207, 212], SUR [150, 207, 212], SE [207], CAUC [212, 231], GEO [117], AZE [277], KAZ [296, 303, 356], RUS-AS: ARC-WSIB [136], ARC-ESIB [164, 212], W-SIB [212, 265], S-SIB [69, 70, 212], E-SIB $[69,212,275]$, ARC-YAK [47, 212, 239], YAK [239], ARC-FE [40, 212], N-FE [94, 140], S-FE $[77,109,212]$

Struckia Müll.Hal. [Plagiotheciaceae]

- enervis (Broth.) Ignatov, T. J. Kop. \& D.G.Long (Cephalocladium zerovii Laz., Struckia argentata Müll.Hal. subsp. zerovii (Laz.) Tan et al., S. zerovii (Laz.) Hedenäs) - RUS-AS: S-SIB [69, 70, 212] — $\{187\}$

Syntrichia Brid. [Pottiaceae], see also Tortula mucronifolia, T. subulata, etc.

- calcicola J.J.Amann (S. densa (Velen.) J.-P.Frahm, Tortula calcicolens W.A.Kramer, T. densa (Velen.) J.-P.Frahm, T. ruralis var. calcicola (J.J.Amann) Barkman, T. ruralis var. densa Velen.) - EST [237], UKR: C [66], M [66], RUS-EUR: C [212, 345], GEO [117], ARM [297], KAZ [296, 356], TAD [294, 295], KYR [296] - $\{188\}$

- caninervis Mitt. (Tortula caninervis (Mitt.) Broth.) - UKR: M [66], K [66, 328], RUS-EUR: C [207, 212, 345], S-UR [150, 207], SE [207, 212], GEO [117], ARM [297], AZE [277], KAZ [23, 295, 296], TUR [295, 296], UZB [295, 296], TAD [294, 295, 296], KYR [295, 296, 351], RUS-AS: S-SIB [70, 197], E-SIB [162]

- caninervis var. astrakhanica Ignatov, Ignatova \& Suragina - RUS-EUR: SE [207, 212, 213]

- ferganensis (Laz.) Laz. - KAZ [296, 356], TAD [295], KYR [267, 295, 296]

- handelii (Schiffn.) S.Agnew \& Vondr. (S. montana subsp. handelii (Schiffn.) Podp., Tortula handelii Schiffn., T. intermedia subsp. handelii (Schiffn.) Wijk \& Margad.) - UKR: M [66], KAZ [296, 348, 356], TUR [11, 296], TAD [295], KYR [296]

- handelii var. ferganensis (Laz.) Ochyra - UZB [257]

- laevipila Brid. (S. pagorum (Milde) J.J.Amann, Tortula pagorum (Milde) De Not., T. laevipila (Brid.) Schwägr.) - RUS-EUR: KLN [309], CAUC [232, 250], GEO [117], ARM [297], KAZ [296, 356], TAD [295], KYR [267, 296], RUS-AS: S-SIB [70, 212], E-SIB [162, 212, 386], YAK [239], S-FE [76, $212]-\{189\}$

- latifolia (Bruch ex Hartm.) Huebener (Tortula latifolia Bruch ex Hartm.) - LAT [3], ?BEL [355], UKR: C [66, 413], RUS-EUR: KLN [309], RUS-AS: YAK [239]

- montana Nees (S. intermedia Brid.) - UKR: C [66], M [66], K [66, 328], RUS-EUR: S-UR [207, 212], SE [207], CAUC [55, 212, 232], GEO [102, 117], ARM [297], AZE [277], TAD [294, 295]

- norvegica F.Weber (Tortula norvegica (F.Weber) Lindb.) - EST [237], UKR: C [66], M [66], K [66, 328], RUS-EUR: ARC [47, 150], NW [87, 273, 364, 407], NE [207, 212, 415], N-UR [90, 150, 207], S-UR [150, 207], CAUC [55, 212, 231, 248], GEO [117], ARM [297], KAZ [296, 356], TAD [294, 295], UZB [267], KYR [267, 296, 351], RUS-AS: ARC-WSIB [136], ARC-ESIB [164, 212, 275], SSIB [69, 70, 212], E-SIB [212, 275], ARC-YAK [47], YAK [239], ARC-FE [40], N-FE [140], S-FE $[67,212]$

- papillosa (Wilson) Jur. (Tortula papillosa Wilson) - LTV [244], UKR: C [66], M [66], RUS-EUR: KLN [309], CAUC [226], GEO [117], AZE [277]

- papillosissima (Copp.) Loeske (S. ruralis var. hirsuta (Venturi) Podp., Tortula hirsuta (Venturi) Laz., T. papillosissima (Copp.) Broth., T. ruralis subsp. hirsuta (Venturi) W.A.Kramer, T. ruralis var. hirsuta (Venturi) Paris) - GEO [117], AZE [277], KAZ [295, 296, 356, 348], TUR [295, 296], TAD [294, 295], KYR [296, 349, 351]

— princeps (De Not.) Mitt. (Tortula princeps De Not.) - GEO [117], ARM [297], TUR [257, 267, 295, 296], UZB [296], TAD [294, 295], KYR [296]

- princeps var. parnassica (Schiffn.) Podp. - TUR [257]

— pseudohandelii (J.Fröhl.) S.Agnew \& Vondr. - TUR [257], UZB [257], TAD [257]

- ruralis (Hedw.) F.Weber \& D.Mohr (Tortula ruralis (Hedw.) P.Gaertn., B.Mey. \& Scherb.) - LTV [244], LAT [3], EST [237], BEL [355], UKR: C [66], M [66], K [66, 328], MLD [367], RUS-EUR: ARC [47, 127, 150], NW [207, 212, 364, 407], NE [207, 212, 415], KLN [309], C [207, 212], N-UR [150, 207, 212], S-UR [150, 207, 212], SE [207, 212], CAUC [55, 212, 231, 248], GEO [117], ARM 
[297], AZE [277], KAZ [156, 295, 296, 356], TUR [295, 296], UZB [295, 296], TAD [294, 295, 296], KYR [295, 296, 351], RUS-AS: ARC-WSIB [136], ARC-ESIB [164, 212, 275], S-SIB [69, 70, 212], E-SIB [69, 212, 275], ARC-YAK [47, 212, 239], YAK [212, 239], ARC-FE [40, 212], N-FE [94, 140], S-FE [67]

- ruralis var. ruraliformis (Besch.) Delogne (S. ruraliformis (Besch.) Cardot, S. ruralis var. arenicola J.J.Amann, nom. illeg., Tortula ruraliformis (Besch.) Ingham) - LAT [1], EST [237], BEL [355], UKR: C [66], M [66], K [66, 328], RUS-EUR: C [345], KLN [309], GEO [117, 257], ARM [297], AZE [277], KAZ [159], TUR [368], TAD [257, 294, 295], KYR [257, 296, 351]

- ruralis var. subpapillosissima (Bizot \& R.B.Pierrot) R.H.Zander - GEO [257],

- sinensis (Müll.Hal.) Ochyra (Tortula sinensis (Müll.Hal.) Broth.) - UKR: C [66, 413], K [328], RUSEUR: CAUC [212, 231, 248], GEO [117, 207], ARM [297], AZE [277], KAZ [393], TUR [296], TAD [294, 295], KYR [267, 296], RUS-AS: S-SIB [69, 70, 212], E-SIB [212, 386], S-FE [76, 212]

- submontana (Broth.) Ochyra - TAD [295], KYR [257, 296]

- virescens (De Not.) Ochyra (Tortula virescens (De Not.) De Not., T. pulvinata (Jur.) Limpr.) - LTV [244], LAT [3], EST [237], BEL [355], UKR: C [66], M [66], K [66, 328], RUS-EUR: KLN [309], C [207, 345], S-UR [207, 212], SE [207], CAUC [232], GEO [117], ARM [297], AZE [277], KAZ [393], TUR [296], TAD [295], KYR [296, 351]

Taxiphyllum M.Fleisch. [*Hypnaceae]

— alternans (Cardot) Z.Iwats. - RUS-AS: S-FE [76]

- aomoriense (Besch.) Z.Iwats. - RUS-AS: S-FE [76, 77, 109, 212]

— densifolium (Lindb. ex Broth.) Reimers - RUS-EUR: CAUC [208, 212, 232], GEO [117], AZE [277]

- taxirameum (Mitt.) M.Fleisch. - RUS-AS: S-FE [76, 212]

— wissgrillii (Garov.) Wijk \& Margad. - LAT [3], EST [237], UKR: C [66], M [66], K [66, 328], MLD [367], RUS-EUR: NW [207, 407], KLN [309], C [207, 212, 345], N-UR [90, 150, 207, 212], S-UR [150, 207, 212], SE [207], CAUC [55, 212, 231, 248], GEO [117], ARM [297], AZE [277], RUS-AS: S-SIB [70, 212], YAK [239]

Tayloria Hook. [Splachnaceae]

- acuminata Hornsch. - RUS-EUR: ARC [47], NE [415], CAUC [248], KAZ [157, 159], TAD [294, 296], KYR [296], RUS-AS: ARC-ESIB [39, 212], S-SIB [69, 70, 212], ARC-YAK [47, 239], YAK [239], ARC-FE [40]

- froelichiana (Hedw.) Mitt. ex Broth. - GEO [102], KAZ [157, 159, 356], RUS-AS: S-SIB [69, 70, 212], ARC-FE [40], N-FE [40]

- hornschuchii (Grev. \& Arn.) Broth. - RUS-AS: ARC-FE [40, 212], KAZ [157]

- lingulata (Dicks.) Lindb. - UKR: C [66], RUS-EUR: ARC [47, 127, 150], NW [212, 273, 364, 407], N-UR [90, 150, 212], CAUC [55, 212], TAD [295], RUS-AS: ARC-WSIB [136], ARC-WSIB [47, 136], ARC-ESIB [275], S-SIB [69, 70, 212], E-SIB [212, 275], ARC-YAK [47, 239], YAK [239], ARC-FE [40, 212], N-FE [40, 140]

- serrata (Hedw.) Bruch et al. - UKR: C [66], RUS-EUR: NW [207], S-UR [150, 207, 212], CAUC [212, 231, 248], KAZ [157, 296, 356], TAD [294, 296], KYR [296], RUS-AS: N-FE [40]

- splachnoides (Schleich. ex Schwägr.) Hook. - RUS-EUR: NW [364, 407], S-UR [150, 207, 212], CAUC [248], GEO [31], RUS-AS: ARC-YAK [47, 239], N-FE [140] + MID-AS [296]

- tenuis (Dicks. ex With.) Schimp. (T. serrata var. tenuis (Dicks.) Bruch et al.) - LTV [244], LAT [3], EST [237], UKR: C [66], RUS-EUR: ARC [150, 415], NW [207, 212, 364, 407], C [207], NE [415], N-UR [207], S-UR [207], KAZ [157, 296, 356], KYR [296, 351], RUS-AS: S-SIB [69, 70], E-SIB [69], ARC-YAK [47, 212, 239], ARC-FE [40]

Tetraphis Hedw. [Tetraphidaceae]

- geniculata Girg. ex Milde - RUS-AS: S-FE [76, 77, 109, 212, 275]

— pellucida Hedw. - LTV [244], LAT [3], EST [237], BEL [355], UKR: C [66], M [66], RUS-EUR: ARC [47, 127, 150], NW [207, 212, 364, 407], NE [207, 212, 415], KLN [309], C [207, 212], N-UR [150, 207, 212], S-UR [150, 207, 212], SE [207, 212], CAUC [55, 208, 212, 231], GEO [117], ARM [297], AZE [277], KAZ [296, 356], TAD [295], RUS-AS: W-SIB [212, 265], S-SIB [69, 70, 212], ESIB [69, 212], YAK [212, 239], N-FE [40, 94, 140], S-FE [76, 77, 109, 212] 
Tetraplodon Bruch et al. [Splachnaceae]

- angustatus (Hedw.) Bruch et al. - LAT [3], UKR: C [66], RUS-EUR: ARC [47, 150], NW [207, 212 , 364, 407], NE [212, 288, 415], C [207, 212], N-UR [90, 150, 415], S-UR [150, 207, 212], CAUC [55, 212], KAZ [296, 356], RUS-AS: ARC-WSIB [136], W-SIB [212, 265], S-SIB [69, 70, 212], E-SIB $[69,212,275]$, YAK [212, 239], ARC-FE [40], N-FE [140, 237], S-FE [109, 212, 221]

- mnioides (Hedw.) Bruch et al. - EST [237], UKR: C [66], RUS-EUR: ARC [47, 127, 150], NW [207, 212, 364, 407], NE [207, 212, 415], N-UR [150, 207, 212], S-UR [150, 207, 212], KAZ [296, 356], KYR [296], RUS-AS: ARC-WSIB [136], ARC-ESIB [164, 212, 275], W-SIB [265], S-SIB [69, 70, 212], E-SIB [69, 212, 275], ARC-YAK [47, 212, 239], YAK [212, 239], ARC-FE [40, 212], N-FE [94, 140], S-FE [76, 212, 221]

— pallidus I.Hagen - RUS-EUR: ARC [150], RUS-AS: ARC-ESIB [212], E-SIB [126, 212], ARC-FE [40], N-FE [40]

- paradoxus (R.Br.) I.Hagen - RUS-EUR: ARC [47, 150, 415], N-UR [150], RUS-AS: ARC-WSIB [136], ARC-ESIB [212], S-SIB [70, 212], E-SIB [126, 212], ARC-YAK [47, 212, 239], YAK [239], ARC-FE [40], N-FE [40]

- urceolatus (Hedw.) Bruch et al. - RUS-EUR: ?NE [415], CAUC [231], GEO [102, 117], KAZ [159], RUS-AS: ARC-WSIB [136], ARC-ESIB [212], S-SIB [70, 212], ARC-YAK [47, 239], YAK [239], ARC-FE [40, 212], N-FE [40, 140] + MID-AS [296]

Tetrodontium Schwägr. [Tetraphidaceae]

— brownianum (Dicks.) Schwägr. - UKR: C [66], RUS-AS: S-SIB [69, 70, 212], E-SIB [69], YAK [239]

— ovatum (Funck) Schwägr. - UKR: C [413] - \{190\}

— repandum (Funck) Schwägr. - RUS-EUR: NW [82], GEO [14, 117], RUS-AS: S-FE [115, 212, 221]

Thamnium see Thamnobryum

Thamnobryum Nieuwl. [Neckeraceae]

- alopecurum (Hedw.) Gang. - LAT [3], EST [237], BEL [355], UKR: C [66], M [66], K [66, 328], RUS-EUR: NW [207], KLN [309], CAUC [55, 208, 212, 232, 248], GEO [117], ARM [297], AZE [277], RUS-AS: N-FE [140]

- coreanum (Cardot) Nog. \& Z.Iwats. - RUS-AS: S-FE [78, 312]

- neckeroides (Hook.) E.Lawton - RUS-AS: S-SIB [70, 212], E-SIB [69, 212, 275], S-FE [76, 212]

— plicatulum (Sande Lac.) Z.Iwats. - RUS-AS: S-FE [76, 109, 212]

- subseriatum (Mitt. ex Sande Lac.) B.C.Tan (T. sandei (Besch.) Z.Iwats.) - RUS-AS: S-FE [76, 77, 212]

- vorobjovii (Laz.) Ochyra - RUS-AS: S-FE [76, 212]

Thuidium Bruch et al. [Thuidiaceae], see also Abietinella abietina, Bryonoguchia molkenboeri, Pelekium minutulum, $P$. pygmaeum, $P$. versicolor, Rauiella fujisana $-\{191\}$

- assimile (Mitt.) A.Jaeger (T. philibertii Limpr.) - LTV [244], LAT [3], EST [237], BEL [355], UKR: C [66], M [66], K [66, 328], MLD [367], RUS-EUR: ARC [150, 415], NW [207, 407], NE [207, 212], KLN [309], C [207, 212], N-UR [90, 150, 207, 212], S-UR [150, 207, 212], SE [207], CAUC [55, 212, 231, 248], GEO [117], ARM [297], AZE [277], KAZ [159, 296, 356], KYR [296], RUS-AS: ARCWSIB [136], ARC-ESIB [164, 212], W-SIB [265], S-SIB [69, 70, 212], E-SIB [69, 212], ARC-YAK [47, 212, 239], YAK [212, 239], ARC-FE [40, 212], N-FE [94, 140], S-FE [76, 77, 109, 212] - \{192\}

- cymbifolium (Dozy \& Molk.) Dozy \& Molk. - RUS-AS: S-FE [76, 212]

- delicatulum (Hedw.) Bruch et al. - LTV [244], LAT [3], EST [237], BEL [355], UKR: C [66], M [66], MLD [367], RUS-EUR: NW [207, 407], NE [207, 415], KLN [309], C [207, 212, 345], S-UR [150, 212], SE [366], CAUC [55, 212, 231, 248], GEO [117], ARM [297], AZE [277], RUS-AS: SSIB [69, 70, 212], E-SIB [212, 275], YAK [239], S-FE [76, 77, 212]

- kanedae Sakurai - RUS-AS: S-FE [76, 77]

— pristocalyx (Müll.Hal.) A.Jaeger (T. glaucinum (Mitt.) Bosch \& Sande Lac.) - RUS-AS: S-FE [76, 212]

- recognitum (Hedw.) Lindb. - LTV [244], LAT [3], EST [237], BEL [355], UKR: C [66], M [66], K [66, 328], MLD [367], RUS-EUR: ARC [47, 150], NW [207, 212, 364, 407], NE [207, 212, 415], KLN [309], C [207, 212], N-UR [90, 150, 207, 212], S-UR [150, 207, 212], SE [207], CAUC [55, 212, 232, 
248], GEO [117], ARM [297], AZE [277], KAZ [159], KYR [296], RUS-AS: W-SIB [265], S-SIB [69, 70, 212], E-SIB [212, 275], ARC-YAK [47, 239], YAK [212, 239], ARC-FE [40], N-FE [40, 140]

- subglaucinum Cardot - RUS-AS: S-FE [116]

- submicropteris Cardot - RUS-AS: S-FE [76, 212]

- tamariscinum (Hedw.) Bruch et al. - LTV [244], LAT [3], EST [237], BEL [355], UKR: C [66], M [66], K [328], RUS-EUR: NW [207], KLN [309], C [207], ?S-UR [150], CAUC [55, 208, 212], GEO [117], ARM [297], AZE [277], RUS-AS: S-FE [77, 109, 212] - \{193\}

- thermophilum Czernyadjeva - RUS-AS: N-FE [142]

Timmia Hedw. [Timmiaceae]

- austriaca Hedw. (T. austriaca var. arctica (Lindb.) Arnell) - UKR: C [66], RUS-EUR: ARC [47, 127, 150], NW [212, 364, 407], NE [207, 212, 415], N-UR [150, 212], S-UR [150, 207], CAUC [22], GEO [117], ARM [297], RUS-AS: ARC-WSIB [136], ARC-ESIB [164, 212], S-SIB [69, 197], E-SIB [212, 275], ARC-YAK [47, 212, 239], YAK [212, 239], ARC-FE [40, 212], N-FE [94, 140]

- bavarica Hessl. - EST [237], BEL [355], UKR: C [66], M [66], K [66, 328], MLD [367], RUS-EUR: NW [364, 407], NE [207, 212, 415], C [207, 212, 345], N-UR [150, 207, 212], S-UR [150, 207, 212], CAUC [55, 212, 231, 248], GEO [117], ARM [297], AZE [277], KAZ [156, 296, 356], TUR [296], TAD [267, 294, 296], KYR [267, 296, 349], UZB [267], RUS-AS: ARC-WSIB [136], W-SIB [212, 265], S-SIB [69, 70, 212], E-SIB [69, 212], ARC-YAK [47, 239], YAK [212, 239], ARC-FE [40, 212], N-FE [40], S-FE [37, 114, 212]

- comata Lindb. \& Arnell - RUS-EUR: ARC [47, 150], NW [364, 407], NE [212, 415], N-UR [150, 207, 212, 415], S-UR [177], CAUC [22], GEO [117], RUS-AS: ARC-ESIB [164, 212, 275], W-SIB [307], S-SIB [69, 70, 212, 275], E-SIB [212, 275], ARC-YAK [47, 212, 239], YAK [212, 239], ARCFE [40, 212], N-FE [140]

- megapolitana Hedw. - EST [237], BEL [355], UKR: C [66], M [66], K [328], RUS-EUR: NW [207], NE [207, 212, 415], C [207, 212, 345], N-UR [90, 207], S-UR [150, 207], SE [207], GEO [117], ARM [297], TUR [11], RUS-AS: ARC-ESIB [212], W-SIB [212, 265], S-SIB [69, 70, 212], E-SIB $[212,275]$, ARC-YAK [47, 239], YAK [212, 239], N-FE [140], S-FE [76, 212, 221] - \{194\}

- norvegica J.E.Zetterst. - RUS-EUR: ARC [47, 150], NE [415], CAUC [55, 212], GEO [117], KAZ [159, 296, 356], RUS-AS: ARC-ESIB [164, 212], S-SIB [69, 70, 212], E-SIB [212, 386], ARC-YAK [47, 212, 239], YAK [239], ARC-FE [40, 212], S-FE [77, 212, 221]

- sibirica Lindb. \& Arnell - RUS-AS: ARC-ESIB [39, 164, 212], E-SIB [212, 275], YAK [239], ARCFE [40, 212]

Timmiella (De Not.) Limpr. [Pottiaceae]

- anomala (Bruch \& Schimp.) Limpr. - GEO [28, 31], RUS-EUR: NE [416], TAD [294, 295], RUSAS: S-SIB [69, 70, 212], S-FE [30, 76]

- barbuloides (Brid.) Mönk. (?T. corniculata (Wahlenb.) Broth.) - TUR [267, 368], RUS-AS: N-FE $[140]-\{195\}$

Tomentypnum Loeske [*Amblystegiaceae]

- falcifolium (Renauld ex Nichols) Tuom. - RUS-AS: S-SIB [255], E-SIB [212, 386], YAK [212, 239], ARC-FE [40, 212]

- nitens (Hedw.) Loeske - LTV [244], LAT [3], EST [237], BEL [355], UKR: C [66], M [66], RUSEUR: ARC [47, 127, 150], NW [207, 212, 364, 407], NE [207, 212, 415], KLN [309], C [207, 212], N-UR [150, 207, 212], S-UR [150, 207, 212], CAUC [248], GEO [117], ARM [297], KAZ [296], RUS-AS: ARC-WSIB [136], ARC-ESIB [164, 212, 275], W-SIB [212, 265], S-SIB [69, 70, 212], ESIB [69, 212, 275], ARC-YAK [47, 212, 239], YAK [212, 239], ARC-FE [40, 212], N-FE [94, 140], SFE [37, 212]

Tortella (Müll.Hal.) Limpr. [Pottiaceae]

— alpicola Dixon - RUS-EUR: NE [166], N-UR [325], S-UR [325], CAUC [231], UZB [325], KYR [325], RUS-AS: ARC-ESIB [164, 325], S-SIB [325], E-SIB [163], YAK [239, 325], ARC-FE [325], N-FE [161], S-FE [114]

- $\operatorname{arctica}$ (Arnell) Crundw. \& Nyholm - RUS-AS: ARC-ESIB [164, 212], E-SIB [126, 212], ARC-YAK [47, 239], YAK [212, 239], ARC-ESIB [50], ARC-FE [40, 212], N-FE [140] 
- bambergeri (Schimp.) Broth. - RUS-EUR: CAUC [226]

- ?brotheri (Lindb. ex Broth.) Broth. - GEO [102] - \{196\}

- flavovirens (Bruch) Broth. - RUS-EUR: CAUC [232]

- fragilis (Hook. \& Wilson) Limpr. - LTV [244], LAT [3], EST [237], UKR: K [328], RUS-EUR: ARC [47, 150, 415], NW [212, 273, 364, 407], NE [207, 212], C [212, 304], N-UR [150, 207, 212], S-UR [150, 207, 212], CAUC [212, 231, 248], GEO [117], ARM [297], AZE [277], KAZ [156, 159, 296, 356], TUR [296], TAD [294, 295], KYR [267, 296, 349, 351], RUS-AS: ARC-WSIB [136], ARCESIB [164, 212, 275], W-SIB [265], S-SIB [69, 70, 212], E-SIB [69, 212], ARC-YAK [47, 212, 239], YAK $[212,239]$, ARC-FE [40, 212], N-FE [94, 140], S-FE [77, 109, 212] - \{197\}

- humilis (Hedw.) Jenn. - UKR: C [66, 413], M [66], K [66, 328], RUS-EUR: S-UR [150], GEO [102]

- inclinata (R.Hedw.) Limpr. - LTV [244], LAT [3], EST [237], UKR: C [66], M [66], K [66, 328], RUS-EUR: NE [415], S-UR [91], CAUC [226], GEO [117], AZE [277], TAD [295], RUS-AS: S-SIB $[69,70]$, ARC-YAK $[47,36]$, YAK [239]

- nitida (Lindb.) Broth. - KAZ [59]

— rigens Alberts. - EST [237]

- tortuosa (Hedw.) Limpr. - LTV [244], LAT [3], EST [237], BEL [355], UKR: C [66], M [66], K [66, 328], MLD [367], RUS-EUR: ARC [47, 127, 150], NW [212, 273, 364, 407], NE [207, 212, 415], C [207, 212, 345], N-UR [150, 207, 212], S-UR [150, 207, 212], CAUC [55, 208, 212, 231, 248], GEO [117], ARM [297], AZE [277], KAZ [156, 267, 296, 356], TUR [295], TAD [294, 295], KYR [267, 296, 349, 351], RUS-AS: ARC-WSIB [136], ARC-ESIB [164, 212], S-SIB [69, 70, 212], E-SIB [212, 275], ARC-YAK [47, 212, 239], YAK [212, 239], ARC-FE [40, 212], N-FE [94, 140], S-FE [212, 221]

Tortula Hedw. [Pottiaceae], see also Syntrichia laevipila, S. ruralis, S. virescens, etc., Hilpertia velenovskyi

— acaulon (With.) R.H.Zander (Phascum cuspidatum Hedw., P. piliferum Hedw.) - LTV [244], LAT [3], EST [237], BEL [355], UKR: C [66], M [66], K [66, 328], MLD [367], RUS-EUR: NW [207], KLN [309], C [207, 212, 345], N-UR [150], S-UR [150, 207], SE [207, 212], CAUC [212, 231, 248], GEO [117], ARM [297], AZE [277], TUR [267, 296], UZB [296], TAD [294, 295], KYR [296, 349, 351], RUS-AS: W-SIB [265], S-SIB [69], YAK [239], ARC-FE [40], N-FE [40]

- afanassievii Laz. - TAD [295]

— altaica Sakauova \& Mamatkulov, nom. nud. - KAZ [296]

— altipes (Broth.) R.H.Zander - TAD [295], KYR [295]

- atrovirens (Sm.) Lindb. - RUS-EUR: CAUC [102, 212, 231, 248, 250], GEO [117], ARM [297], TUR [11, 296], UZB [295], TAD [295], KYR [296]

- brevissima Schiffn. - TUR [11, 296], KYR [351], TAD [295]

- canescens Mont. - UKR: C [66, 413], M [66], K [328], ARM [342], TUR [267, 296]

- caucasica Lindb. ex Broth. (Pottia caucasica (Lindb. ex Broth.) Paris) - GEO [102]

- cernua (Huebener) Lindb. (Desmatodon cernuus (Huebener) Bruch et al.) - LAT [3], UKR: M [66], RUS-EUR: NW [85, 212, 364], C [207], KAZ [295], TAD [295], KYR [296, 351], RUS-AS: S-SIB [69, 70, 212], E-SIB [163], ARC-YAK [47, 239], YAK [239], ARC-FE [40], N-FE [77, 140]

- cuneifolia (Dicks.) Turner - BEL [355], UKR: M [66], KAZ [157], UZB [295], TAD [294, 295], KYR [296]

- fiori (Venturi) G.Roth - TUR [11] - $\{197\}$

— grandiretis Broth. - TUR [296], UZB [267, 296, 385], TAD [296], KYR [296]

- hoppeana (Schultz) Ochyra (Desmatodon latifolius (Hedw.) Brid., Tortula eurhyphylla R.H.Zander UKR: C [66], M [66], RUS-EUR: ARC [47, 127, 150, 415], NW [212, 273, 364, 407], C [207, 345], N-UR [150, 207, 212, 415], S-UR [150, 207], CAUC [55, 212, 231, 248], GEO [117], ARM [297], AZE [277], KAZ [296, 356], TAD [294, 295], UZB [267], KYR [296, 349, 351], RUS-AS: ARCWSIB [136], ARC-ESIB [39, 164], S-SIB [69, 70, 212], E-SIB [69], ARC-YAK [47, 239], YAK [239], ARC-FE [40, 212], N-FE [94, 140], S-FE [114, 212, 221]

- inermis (Brid.) Mont. (Syntrichia inermis (Brid.) Bruch) - UKR: K [328], RUS-EUR: S-UR [150], CAUC [231, 232, 250], GEO [117], ARM [297], AZE [277], KAZ [393], TUR [267, 295, 296], UZB [267, 296], TAD [294, 295], KYR [267, 296, 349, 351] 
- lanceola R.H.Zander (Pottia lanceolata (Hedw.) Müll.Hal.) - LTV [244], EST [237], BEL [355], UKR: C [66, 413], M [66], K [66, 328], MLD [367], RUS-EUR: KLN [309], C [207, 345], SE [207, 212, 377], CAUC [232], GEO [117], ARM [297], AZE [277], KAZ [157], TUR [296]

- laureri (Schultz) Lindb. (Desmatodon laureri (Schultz) Bruch et al.) - KAZ [157, 296, 356], TAD [295], KYR [296], RUS-AS: ARC-ESIB [246], S-SIB [69, 70, 212], E-SIB [163], ARC-YAK [47, 239], ARC-FE [40, 212]

— lazarenkoi L.I.Savicz - TUR [296], TAD [295], KYR [267, 295, 296]

- leucostoma (R.Br.) Hook. \& Grev. (Desmatodon leucostoma (R.Br.) Berggr., D. suberectus (Drumm.) Limpr.) - RUS-EUR: ARC [47, 150, 415], KAZ [296, 356], TAD [294, 295], KYR [296], RUS-AS: ARC-ESIB [164, 212], S-SIB [70, 212], E-SIB [163], ARC-YAK [47, 239], YAK [239], ARC-FE [40, 212], N-FE [40]

- lindbergii Kindb. (Pottia lindbergii (Kindb.) Warnst.) - GEO [102]

— lingulata Lindb. - LAT [3], EST [237], UKR: M [66], RUS-EUR: NW [207], C [207], CAUC [248], GEO [117], TAD [294]

- marginata (Bruch et al.) Spruce (Desmatodon meridionalis Luisier) - TAD [34, 295]

- modica R.H.Zander (Pottia intermedia (Turner) Fürnr.)- LTV [244], LAT [3], EST [237], BEL [355], UKR: C [66], M [66], K [66, 328], RUS-EUR: NW [207], KLN [309], C [207, 345], S-UR [150, 207], SE [207], GEO [117], RUS-AS: W-SIB [265], S-SIB [424]

- mucronifolia Schwägr. - UKR: M [66], K [66, 328], RUS-EUR: ARC [47, 150], NW [364, 407], NE [207, 212, 415], C [207, 212, 345], N-UR [90, 150, 207], S-UR [150, 207], SE [207, 212], CAUC [55, 212, 248], GEO [117], ARM [297], AZE [277], KAZ [156, 159, 296], TUR [296], TAD [294, 295], KYR [267, 296, 351], RUS-AS: ARC-WSIB [136], ARC-ESIB [164, 212, 275], W-SIB [212, 265], SSIB [69, 70, 212], E-SIB [212, 275], ARC-YAK [47, 212, 239], YAK [239], ARC-FE [40, 212], N-FE [94, 140], S-FE [76]

- muralis Hedw. - LTV [244], LAT [3], EST [237], BEL [355], UKR: C [66], M [66], K [66, 328], MLD [367], RUS-EUR: NW [207], NE [207, 212], KLN [309], C [207, 212], S-UR [150, 207], SE [207, 212], CAUC [208, 212, 231, 248], GEO [117], ARM [297], AZE [277], KAZ [159, 295, 296], TUR [295, 296], TAD [294, 295], KYR [267, 296, 351], RUS-AS: S-SIB [69, 70], E-SIB [162], YAK [239]

- muralis var. aestiva Hedw. - EST [237], BEL [355], UKR: C [66], M [66], RUS-EUR: C [207, 212], N-UR [207], S-UR [207, 212], CAUC [231], GEO [117], ARM [297], AZE [277], TUR [295, 296], TAD [295], KYR [296], RUS-AS: YAK [239]

- obtusifolia (Schwägr.) Mathieu - LTV [244], RUS-EUR: C [207, 212], S-UR [150, 207], CAUC [102], GEO [117], ARM [297], AZE [277], KAZ [157], UZB [267, 295, 296], TAD [294, 295], KYR [267, 296], RUS-AS: W-SIB [265], S-SIB [70, 212], E-SIB [126, 212], YAK [239], N-FE [40, 140]

- paulsenii Broth. - TAD [295]

— protobryoides R.H.Zander (Pottia bryoides (Dicks.) Mitt., Protobryum bryoides (Dicks.) J.Guerra \& M.J.Cano) - LTV [244], LAT [3], EST [237], UKR: C [66], M [66], MLD [367], RUS-EUR: NW [207], NE [415], KLN [309], C [207, 345], S-UR [150, 207], SE [207, 212], ARM [297], AZE [277], TUR [296], TAD [294, 295], KYR [349, 351]

- raddei Broth. - TUR [295], TAD [295]

- randii (Kenn.) R.H.Zander (Desmatodon oxneri Laz., D. randii (Kenn.) Laz.) - LAT [3], EST [237], UKR: M [66]

— revolutifolia Laz. - TUR [267, 295, 296], UZB [267, 295], TAD [295], KYR [267, 296]

- revolvens (Schimp.) G.Roth - TUR [296], TAD [295]

- subulata Hedw. - LTV [244], LAT [3], EST [237], BEL [355], UKR: C [66], M [66], K [66, 328], MLD [367], RUS-EUR: KLN [309], C [207, 212, 345], SE [207], CAUC [55, 212, 231, 248], GEO [117], ARM [297], AZE [277], KAZ [157, 159, 296, 356], TUR [295, 296], UZB [295, 296], TAD [294, 295, 296], KYR [267, 296]

- systylia (Schimp.) Lindb. (Desmatodon systylius Schimp.) - RUS-EUR: CAUC [212, 231], GEO [117], KAZ [157], TAD [295], KYR [296, 351], RUS-AS: S-SIB [69, 70, 197, 212], ARC-YAK [47, 239], YAK [239], ARC-FE [40, 212], N-FE [140] 
— thianschanica Broth. - KAZ [59], TUR [296], UZB [385], TAD [294, 295], KYR [267, 295, 296]

- trachyphylla Broth. (Semibarbula trachyphylla (Broth.) Laz. ex Mamatkulov) - TUR [267, 296], KAZ [59], UZB [296], TAD [294, 295, 296], KYR [296] - \{198\}

- transcaspica Broth. - TUR [295, 296], UZB [385], TAD [295]

- truncata (Hedw.) Mitt. (Pottia truncata (Hedw.) Bruch et al.) - LTV [244], LAT [3], EST [237], BEL [355], UKR: C [66], M [66], K [66, 328], MLD [367], RUS-EUR: NW [207, 407], NE [207, 415], KLN [309], C [207, 212], N-UR [150, 207], S-UR [150, 207], SE [207], CAUC [55, 212, 232], GEO [117], AZE [277], RUS-AS: ARC-ESIB [164], W-SIB [265, 275], S-SIB [431], S-FE [76, 212]

- ucrainica (Laz.) R.H.Zander (Desmatodon ucrainicus Laz.) - UKR: M [66], RUS-AS: ?S-FE [76]

- vahliana (Schultz) Mont. - AZE [429]

Trachycystis Lindb. [Mniaceae]

- flagellaris (Sull. \& Lesq.) Lindb. - RUS-AS: N-FE [94, 140], S-FE [76, 77, 109, 212]

- microphyllus (Dozy \& Molk.) Lindb. - RUS-AS: S-FE [76, 212]

- ussuriensis (Maack \& Regel) T.J.Kop. - RUS-EUR: CAUC [55, 212, 248], GEO [117], ARM [297], AZE [277], RUS-AS: S-SIB [69, 197, 212], E-SIB [70, 212], YAK [239], ARC-FE [40], N-FE [140], S-FE [76, 109, 212]

Trematodon Michx. [Bruchiaceae]

- ambiguus (Hedw.) Hornsch. - LTV [244], LAT [3], EST [237], BEL [355], UKR: M [66], RUSEUR: NW [207, 273, 364, 407], NE [90, 207, 212, 416], KLN [309], C [207, 212, 345], CAUC [231], GEO [117], RUS-AS: ARC-FE [40], N-FE [40, 140], S-FE [76, 77, 110]

- brevicollis Hornsch. - RUS-EUR: NW [364], KAZ [104], KYR [104, 296], RUS-AS: S-SIB [69, 70]

- longicollis Michx. - RUS-AS: N-FE [235], S-FE [212, 221]

Trichodon see Ditrichum cylindricum

Trichostomopsis see Didymodon

Trichostomum Bruch [Pottiaceae], see also Oxystegus tenuirostris

- arcticum Kaal. - RUS-AS: ARC-ESIB [164, 212], S-SIB [69, 70, 212], E-SIB [69, 212], ARC-YAK [47, 239], YAK [212, 239], ARC-FE [40], N-FE [40, 94]

- brachydontium Bruch - EST [396], UKR: C [66], K [66, 328], RUS-EUR: CAUC [208, 212, 232, 248], GEO [117], RUS-AS: S-FE [340] - \{199\}

- connivens (Lindb. ex Broth.) Paris - RUS-EUR: CAUC [102], GEO [117]

- crispulum Bruch (T. brevifolium Sendtn. ex Müll.Hall., T. viridulum Bruch) - LTV [244], LAT [3], EST [237], UKR: C [66], K [66, 328], RUS-EUR: NW [207], NE [207, 212, 415], C [207, 212, 345], N-UR [90, 150, 207, 212], S-UR [207, 212], SE [207], CAUC [55, 212, 231, 248], GEO [117], ARM [297], AZE [277], TAD [294, 295], RUS-AS: ARC-WSIB [136], ARC-ESIB [164, 212, 275], E-SIB $[163,275]$, YAK [239], ARC-FE [40], N-FE [40], S-FE [76, 212]

- triumphans De Not. (Weissia triumphans (De Not.) M.O.Hill) - TUR [11, 296]

— viridulum Bruch - UKR: C [66], K [66, 328], AZE [277]

Tuerckheimia svihlae (E.B.Bartram) R.H.Zander - excluded $-\{200\}$

Ulota D.Mohr [Orthotrichaceae]

- bruchii Hornsch. ex Brid. - LAT [3], EST [237], BEL [355], UKR: C [66], RUS-EUR: KLN [309]

- coarctata (P.Beauv.) Hammar - LAT [3], EST [237], BEL [355], UKR: C [66, 413], M [66, 148], RUS-EUR: KLN [309], CAUC [55, 212]

- crispa (Hedw.) Brid. (U. crispula Bruch ex Brid.) - LTV [244], LAT [3], EST [237], BEL [355], UKR: C [66], M [66], K [66, 328], RUS-EUR: NW [100, 207, 212], KLN [309], C [207, 212], CAUC [55, 208, 212, 231, 248], GEO [117], ARM [297], KAZ [296, 356], RUS-AS: S-SIB [70, 212, 217], E-SIB [74, 212, 247], N-FE [140], S-FE [76, 77, 109, 212]

- curvifolia (Wahlenb.) Lilj. - EST [237], RUS-EUR: ARC [47, 150], NW [212, 273, 364, 407], N-UR [150, 212, 416], S-UR [150, 207], CAUC [248], ARM [297], KAZ [296, 356], RUS-AS: S-SIB [69, 70, 212, 217], E-SIB [69, 212], YAK [239], ARC-FE [40, 212], N-FE [40, 94, 95], S-FE [212, 221]

- drummondii (Hook. \& Grev.) Brid. - LAT [4], EST [237], UKR: C [66, 413], RUS-AS: N-FE [140], S-FE [76, 77, 109]

- hutchinsiae (Sm.) Hammar (U. americana (P. Beauv.) Limpr.) - EST [396], UKR: C [66], RUS-EUR: 
NW [407], CAUC [55, 212], AZE [277], RUS-AS: S-SIB [70, 212, 217]

- japonica (Sull. \& Lesq.) Mitt. - RUS-AS: S-FE [77, 109]

- phyllantha Brid. - RUS-EUR: NW [364]

— rehmannii Jur. - UKR: C [66, 413], GEO [117], RUS-AS: S-SIB [197, 212, 217]

- reptans Mitt. - RUS-AS: S-FE [212, 221]

Vesicularia (Müll.Hal.) Müll.Hal. [*Hypnaceae]

— flaccida (Sull. \& Lesq.) Z.Iwats. - RUS-AS: S-FE [76, 212]

Voitia Hornsch. [Splachnaceae]

- hyperborea Grev. \& Arn. - RUS-EUR: ARC [47, 341], RUS-AS: ARC-FE [40]

- nivalis Hornsch. - TAD [296], KYR [296, 351], RUS-AS: S-SIB [69, 70, 212]

Warnstorfia Loeske [Calliergonaceae] $-\{165\}$

- exannulata (Bruch et al.) Loeske (Drepanocladus exannulatus (Bruch et al.) Warnst., Sarmentypnum exannulatum (Bruch et al.) Hedenäs) - LTV [244], LAT [3], EST [237], BEL [355], UKR: C [66], M [66], RUS-EUR: ARC [47, 127, 150], NW [207, 212, 364, 407], NE [207, 212, 415], KLN [309], C [207, 212], N-UR [150, 207, 212], S-UR [150, 207, 212], SE [207, 212], CAUC [55, 212, 231, 248], GEO [117], ARM [297], AZE [277], KAZ [23, 156, 356], TAD [267, 294, 296], KYR [296, 351], RUS-AS: ARC-WSIB [136], ARC-ESIB [164, 212], W-SIB [212, 265], S-SIB [69, 70, 212], E-SIB [69, 212, 275], ARC-YAK [47, 212, 239], YAK [212, 239], ARC-FE [40, 212], N-FE [94, 140], S-FE $[76,77,109,212]$

- fluitans (Hedw.) Loeske (Drepanocladus fluitans (Hedw.) Warnst., D. h-schulzei (Limpr.) Loeske, D. kurilensis Smirnova, Warnstorfia fluitans var. falcata (Sanio ex C.E.O.Jensen) H.A.Crum \& L.E.Anderson, W. h-schulzei (Limpr.) Loeske, W. kurilensis (Smirnova) Schljakov) - LTV [244], LAT [3], EST [237], BEL [355], UKR: C [66], M [66], RUS-EUR: ARC [47, 127, 150], NW [207, 212, 364, 407], NE [207, 212, 415], KLN [309], C [207, 212], N-UR [150, 207], S-UR [150, 207, 212], SE [207, 212], CAUC [55, 212, 248], GEO [117], ARM [297], AZE [277], KAZ [158, 296, 356], TAD [294, 296], KYR [296], RUS-AS: ARC-WSIB [136], ARC-ESIB [164, 212], W-SIB [212, 265], SSIB [69, 212], E-SIB [69], ARC-YAK [47, 212, 239], YAK [212, 239], ARC-FE [40, 212], N-FE [94, 140], S-FE [77, 109, 212, 369]

— procera (Renauld \& Arnell) Tuom. (Drepanocladus procerus (Renauld \& Arnell) Warnst., Sarmentypnum procerum (Renauld \& Arnell) Hedenäs) - EST [237], RUS-EUR: NW [87, 273, 407], RUS-AS: ARC-ESIB [212, 263], ARC-FE [40]

— pseudosarmentosa (Cardot \& Thér.) Tuom. \& T.J. Kop. (Sarmentypnum pseudosarmentosum (Cardot \& Thér.) Hedenäs) - RUS-AS: ARC-FE [40]

— pseudostraminea (Müll.Hal.) Tuom. \& T.J.Kop. (Drepanocladus pseudostramineus (Müll.Hal.) G.Roth) - UKR [66], RUS-EUR: ARC [47, 150], NW [212, 273, 291, 364], NE [414], C [207, 212], N-UR [150, 207, 212], S-UR [150, 207, 212], RUS-AS: ARC-WSIB [136], ARC-ESIB [164, 212], W-SIB [212, 265], S-SIB [197, 212], ARC-YAK [47, 239], ARC-FE [40], N-FE [40, 140]

- sarmentosa (Wahlenb.) Hedenäs (Calliergon sarmentosum (Wahlenb.) Kindb., Sarmentypnum sarmentosum (Wahlenb.) Tuom. \& T.J.Kop.) - RUS-EUR: ARC [47, 127, 150], NW [212, 273, 364, 407], NE [207, 212, 415], N-UR [150, 207, 212], S-UR [150], ?CAUC [231], KAZ [356], RUSAS: ARC-WSIB [136], ARC-ESIB [164, 212, 275], W-SIB [265], S-SIB [69, 70, 212], E-SIB [212, 275], ARC-YAK [47, 212, 239], YAK [212, 239], ARC-FE [40, 212], N-FE [94, 140], S-FE $[212,221]$

- trichophylla (Warnst.) Tuom. \& T.J.Kop. (Drepanocladus trichophyllus (Warnst.) Podp., Sarmentypnum trichophyllum (Warnst.) Hedenäs) - LAT [3], EST [237], RUS-EUR: NW [61, 364, 407], NE [288], RUS-AS: ARC-WSIB [136], YAK [239], S-FE [245]

- tundrae (Arnell) Loeske (Drepanocladus tundrae (Arnell) Loeske, Sarmentypnum tundrae (Arnell) Hedenäs) - LTV [244], LAT [3], EST [237], RUS-EUR: ARC [47], NW [212, 364, 407], RUS-AS: ARC-WSIB [136], ARC-ESIB [164, 212, 275], S-SIB [69, 70], E-SIB [275], ARC-YAK [239], YAK [212, 239], ARC-FE [40], N-FE [140], S-FE [212, 221]

Weissia Hedw. [Pottiaceae]

- brachycarpa (Nees \& Hornsch.) Jur. (Hymenostomum microstomum (Hedw.) R.Br. ex Nees \& Horn- 
sch.) - LTV [244], LAT [3], EST [237], BEL [355], UKR: C [66], M [66], K [66, 328], MLD [367], RUS-EUR: KLN [309], C [207, 212, 345], S-UR [150, 207, 212], SE [207], CAUC [55, 102, 212, 231, 248], GEO [102, 117], ARM [297], KAZ [157, 159, 393], TUR [295], TAD [294, 295], KYR [296], RUS-AS: ARC-ESIB [164], W-SIB [265], S-SIB [69, 70, 212], S-FE [76, 212]

- condensa (Voit) Lindb. (W. tortile (Schwägr.) Müll.Hal.) - UKR: C [66], M [66], K [66, 328], MLD [367], RUS-EUR: CAUC [102, 212, 231], GEO [102, 117], ARM [297], AZE [277], KAZ [157, 159, 296], TUR [267, 296], TAD [295], KYR [267, 296, 351], RUS-AS: S-SIB [70, 212]

- controversa Hedw. - LTV [244], LAT [3], EST [237], BEL [355], UKR: C [66], M [66], K [66, 328], RUS-EUR: NW [207], KLN [309], C [207, 212, 345], S-UR [91, 150, 207, 212], SE [207], CAUC [212, 231, 248], GEO [117], ARM [297], AZE [277], KAZ [23, 157, 159], TUR [296], TAD [294, 295], KYR [296, 351], RUS-AS: W-SIB [265], S-SIB [69, 70, 212], ARC-FE [40], N-FE [140], S-FE $[76,77,212]$

- edentula Mitt. - RUS-AS: S-FE [212, 221]

- exserta (Broth.) P.C.Chen - TUR [296], TAD [295], RUS-AS: S-SIB [197, 212], E-SIB [162]

- fallax Sehlm. (W. controversa var. crispata (Nees \& Hornsch.) Nyholm) - UKR: M [66], K [66, 328], MLD [367], RUS-EUR: CAUC [248], GEO [117], AZE [277], ARM [342], TAD [295]

- krassavinii (Laz.) Laz. ex Ochyra - KAZ [393], TUR [296], TAD [294, 295], KYR [267, 296]

- levieri (Limpr.) Kindb. (Astomum levieri Limpr.) - UKR: [66], K [328], MLD [367], RUS-EUR: C [207], SE [207], CAUC [232], GEO [117], ARM [297]

- longifolia Mitt. (Astomum crispum (Hedw.) Hampe) - UKR: C [66], M [66], K [66, 328], MLD [367], RUS-EUR: NW [207], C [207, 212, 345], S-UR [150, 207], SE [207], CAUC [231], GEO [102, 117], ARM [297], RUS-AS: S-SIB [338]

- papillosissima Laz. - TUR [296], TAD [295]

- planifolia Dixon (W. platyphylla Broth.) - RUS-AS: S-FE [76]

- rostellata (Brid.) Lindb. - UKR: C [66, 413], RUS-EUR: SE [207], CAUC [231]

- rutilans (Hedw.) Lindb. - UKR: C [66, 413], M [66], RUS-EUR: S-UR [150, 373], GEO [117], TAD [295], RUS-AS: S-FE [76, 212]

- squarrosa (Nees \& Hornsch.) Müll.Hal. (Hymenostomum squarrosum Nees \& Hornsch.) - LTV [244], EST [237], RUS-EUR: S-UR [207]

— tyrrhena M.Fleisch. - TUR [267, 295, 296], TAD [294, 295], KYR [267, 296]

- wimmeriana (Sendtn.) Bruch et al. (W. controversa var. wimmeriana (Sendtn.) Blockeel \& A.J.E.Sm.) - RUS-EUR: NW [364], CAUC [212, 231], GEO [102], ARM [297], TUR [296], RUS-AS: S-SIB $[212,334]$

Zygodon Hook. \& Taylor [Orthotrichaceae]

- conoideus (Dicks.) Hook. \& Taylor - excluded - $\{201\}$

— dentatus (Limpr.) Kartt. (Z. viridissimus var. dentatus Limpr.) - UKR: C [403], GEO [117]

- rupestris Schimp. ex Lorentz (Z. viridissimus var. rupestris Hartm.) - LTV [244], LAT [3], UKR: C [403], K [328, 403], RUS-EUR: NW [407], CAUC [232, 250] - $\{200\}$

- sibiricus Ignatov, Ignatova, Z.Iwats. \& B.C.Tan - RUS-EUR: NE [414], C [207, 212, 343], N-UR [150], S-UR [150, 207], RUS-AS: ARC-WSIB [265*], S-SIB [210, 212], E-SIB [210, 212], YAK [239], N-FE [140*], S-FE [114, 210, 212, 221] - \{200\}

- viridissimus (Dicks.) Brid. - LTV [244], LAT [3], EST [237], UKR: C [66], K [66, 328], RUS-EUR: CAUC [18, 102], GEO [117], AZE [277], RUS-AS: S-FE [76] - \{202\} 


\section{ANNOTATIONS}

1. It seems that numerous records of Aloina ericaefolia in publications of the beginning of XX century belong in fact not to $A$. ambigua, but to $A$. rigida.

2. Abramova \& Abramov [22] recorded Anacamptodon splachnoides for Irkutsk Province and Primorsky Territory; Bardunov \& Cherdantseva [76] thought that in the latter region it is likely a misidentification of $A$. latidens; Czernyadjeva (unpubl.) referred both these Asian collections to A. latidens.

3. According to Schljakov [pers. comm.] his record of Andreaea rothii from S-UR maybe belongs to $A$. heinemannii.

4. Hill \& al. [190] accepted Anomobryum concinnatum as a separate species, mainly because of strong differences in sporophyte structure; different authors in Russia are inconsistant in delimitation and distribution of $A$. concinnatum and A. julaceum, sometimes treating them as synonymous; revision of the whole material is obviously needed.

5. In synonymization of Anomodon minor ssp. integerrimus with $A$. minor we follow Granzow-de la Cerda [179] and Iwatsuki [242].

6. Virchenko [399] reidentified Atractylocarpus alpinus from Ukraine (where it was reported by Savicz-Lyubitskaya \& Smirnova [361]) and Russian Far East (where it was reported by Bardunov \& Cherdantseva [77]).

7. Smith [371] synonymized Atrichum rhystophyllum with $A$. angustatum; however Iwatsuki [242] kept them as separate species.

8. According to Cherdantseva (unpubl.), records of Atrichum tenellum from Primorsky Territory [76] are erroneous; some other records from the eastern limit of this species are also doubtful.

9. Aulacomnium androgynum was reported from Asian Russia only from Sakhalin by Sugawara [376]; this record has to be confirmed.

10. Molecular phylogenetic analysis of Pedersen \& Hedenäs [331] revealed that Bardunovia baicalensis is very similar in sequences to Platydictya, thus Hedenäs $\&$ Pedersen [189] transferred it to the latter genus.

11. Corley \& al. [121] put Brachytheciastrum collinum into the synonymy of $B$. fendleri, and some records of the latter species were published e.g. for Middle Asia; the real relationship of these two species, as well as the occurrence of $B$. fendleri outside North America needs further studies.

12. According to Ignatov (in prep.) plants called Brachytheciastrum trachypodium from Siberia and Middle Asia are not identical to the Central European ones and have to be separated to its own species.

13. Mamatkulov [294, 296] also reported for Tadjikistan Brachythecium velutinum var. condensatum Bruch et al.; this taxon is not accepted by recent
European authors [e.g. 190].

14. New system for the family Brachytheciaceae has been suggested by Ignatov \& Huttunen [205].

15. Hoffmann [191] synonymized Brachythecium auriculatum with Palamocladium leskeoides, however Ignatov \& al. [215] demonstrated that this suggestion can not be accepted.

16. According to Cherdantseva (unpubl.), Brachythecium campestre was erroneously reported from the Russian Far East; Hedenäs [186] published useful discussion on the circumscription of this species.

17. Brachythecium caucasicum Thér. was described from Adzharia from a sterile specimen; it belongs to $B$. salebrosum complex, but study of additional material from that area is needed to understand its identity.

18. Record of Brachythecium coruscum from RUS-EUR: N-UR [230], also repeated in [150], is erroneous.

19. Brachythecium erythrorrhizon was reported from various parts of Asian Russia, but all revised specimens were found to be ssp. asiaticum; ssp. erythrorrhizon definitely occurs only in NW European Russia and Baltic countries; however a lot of material remains unrevised.

19a. Brachythecium frigidum is a North American species and its record from Kazakhstan seems to be doubtful.

20. Specimens identified as Brachythecium wichurae were found to belong to $B$. complanatum, a superficially similar species [215]; however the former species can be discovered in the Russian Far East; the earlier name for $B$. wichurae is $B$. garovaglioides Müll.Hal.

21. Records of Brachythecium tommasinii from RUSEUR: NE [415] were found to be erroneous [90].

22. Recent molecular phylogenetic studies of Bryum revealed that this genus is not monophyletic; Bryum s. str. includes species around $B$. argenteum, $B$. dichotomum and $B$. caespiticium, while species around B. capillare, B. pallens, B. pseudotriquetrum, B. purpurascens, $B$. wrightii are more related to Plagiobryum and are better placed in this genus [332], and species of B. alpinum complex into Imbribryum N.Pedersen [330]; we think this will be accepted in the future, but here we retain Bryum s.l. because for quite many taxa we have no definite opinion to which genus they belong.

23. Many species of Bryum from East Europe and North Asia are known solely or almost only from the original collections; they include: $B$. alexandri, B. altaicum, B. amblyphyllum, B. axillare, B. congestiflorum, $B$. crassimucronatum, $B$. cremocarpum, $B$. ehlei, $B$. enisseense, B. leucoglyphodon, B. microcalophyllum, B. mollifolium, B. mucronifolium, B. obtusidens, $B$. pallescens var. microblastum, B. pootonense, B. pallescens var. turkestanicum, $B$. pamirense, B. pamiromucronatum, B. pygmaeomucronatum, B. spinifolium, B. subcalophyllum, B. submucronatum, B. ter- 
skeiense, B. tesselatum, B. timmiostomoides, B. utriculatum, B. vernum, B. zemliae.

24. Recently Holyoak [192] synonymized Bryum amblyodon with $B$. archangelicum; however according to Zolotov [420] these species in Russia lack intermediates and can be accepted.

25. Recently Holyoak [192] synonymized Bryum bryoides and B. purpurascens with B. arcticum; however according to Zolotov [420] two latter species in Russia lack intermediates and can be accepted; Bryum bryoides is rare in Russia, preventing a final decision on this species.

26. Recently Holyoak [192] synonymized Bryum axelblyttii with $B$. calophyllum; however according to Zolotov [420] these species in Russia are rather well delimited and can be accepted.

27. Synonymy of Bryum comense was discussed by Hill et al. [190].

28. Dyachenko [151] reported Bryum stirtonii from Northern Ural and suggested that it is a good species, however Holyoak [192] considered it as a synonym of $B$. elegans.

29. Bryum jeniseense is an orthographical error in Ignatov \& Afonina [199], Savicz-Lyubitskaya \& Smirnova [361], etc.; correct name is $B$. enisseense.

30. Recently Holyoak [192] placed Bryum nitidulum into the synonymy of $B$. intermedium, but here two species are considered as separate ones.

31. The species status of Bryum lapponicum has been confirmed by Schljakov \& Konstantinova [364], but they overlooked that the name is illegitimate and has to be replaced.

32. There is a disagreement between Holyoak [192] and Zolotov [418] on the species independence of $B$. pallescens and B. lonchocaulon (B. cirrhatum); some publications accept $B$. pallescens s.l. (incl. $B$. cirrhatum), they are included under both $B$. pallescens and B. lonchocaulon with asterisk.

33. According to the recent revision by Afonina [40], Bryum neodamense does not occur in RUS-AS: ARC-FE, where it has been reported [e.g. 38, 212]; Holyoak \& Hedenäs [420] provided strong evidence for synonymization of these two species despite their large superficial difference.

34. Bryum tardum is a little known species, sometimes referred to the synonymy of $B$. lonchocaulon; the only record of B. tardum from RUS-EUR (NW [364]) is based on a specimen referred by Zolotov to $B$. creberrimum. Bryum tenuisetum was reported from the Baltic region by Düll [154], but we don't know any exact reference. Bryum planiusculum was synonymized with B. turbinatum by Zolotov [420]. Records of Bryum turbinatum from Chukotka were based on specimens reidentified by Schljakov and Zolotov as B. schleicheri.
35. According to Frisvoll [172] and Ignatova (unpubl.), all records of Bucklandiella (Racomitrium) heterostichum from the Asian Russia, Urals and Middle Asia [e.g. 76, 77, 90, 109, 150, 212, 296] were based on a broad concept of $B$. heterosticha (incl. B. microcarpa and $B$. sudetica), and belong now to other species.

36. The volume of the genus Calliergonella was expanded by Hedenäs [185]; all subsequent molecular phylogenetic data confirmed this suggestion.

37. Hedenäs [187] suggested that the Campylium hispidulum complex should be included in Campylophyllum; molecular phylogenetic analysis of Ignatov \& al. [204] however supports an alternative suggestion of Ochyra \& al. [316] who separate them as a genus of its own.

38. Old records of Campylidium hispidulum from Russia were based on the broad concept of this species; then Crundwell \& Nyholm [124] then considered it as a North American species not occurring in Europe; later Hedenäs [187] found it in Japan; material from Asian Russia obviously needs special revision; differences between $C$. hispidulum and C. sommerfeltii are rather small, so American authors doubt the distinctness of these taxa [e.g. 122].

39. The generic placement of Campylium longicuspis was discussed by Hedenäs [183].

40. According to Hedenäs [187], Campylium squarrosulum is probably close to Amblystegium radicale, and should be better classified in that genus.

41. Many authors did not separate Campylium stellatum and $C$. protensum, so at least some records of $C$. stellatum should be referred to this species in the broad sense, e. g. including C. protensum.

42. The systematic position of Campylostelium (Grimmia) pitardii (=Usmania campylopoda) was discussed by Maier [279].

43. According to Ulychna [cf. 413] the only record of Campylostelium strictum in Ukraine is doubtful.

44. In taxomony and synonymy of Ceratodon heterophyllus we follow Burley \& Pritchard [108].

45. Cinclidium minutifolium was synonymized with $C$. latifolium by Mogensen [300]; T. Koponen (in ,Notae criticae' in H) supported this decision.

46. Specimen called Claopodium crispifolium in LE was reidentified by A.L.Abramova and V.Ya. Cherdantseva as $C$. pellucinerve.

47. Position of Anomodon rostratus within Claopodi$u m$ has been discussed by Ignatov \& al. [204].

48. Record of Cnestum glaucescens from S-UR [150] was erroneous [cf. 207].

49. All Asian records of Codriophorus (Racomitrium) aciculare, according to Bednarek-Ochyra [81], belong to other species.

50. Asian records of Codriophorus (Racomitrium) aquaticus, according to Bednarek-Ochyra [81], be- 
long to other species.

51. There are several collections from Asian Russia that fit Coscinodon humilis Milde in gametophytic characters, but without sporophyte or careful comparison with plants with sporophytes this is only a preliminary identification.

52. Ctenidium capillifolium and Neckeropsis nitidula were reported from South Sakhalin only by Sugawara [376]; their occurrence has to be confirmed.

53. Records of Ctenidium molluscum from Urals [cf. 150] were not confirmed in the herbarium [cf. 207].

54. Preliminary revision of Ignatova (in prep.) of Cynodontium asperifolium-C. fallax complex demonstrates that all records of $C$. fallax from Asian Russia and Urals belong in fact to $C$. asperifolium; it seems that $C$. fallax occurs in Russia only in Caucasus.

55. Werner [409] demonstrated numerous differences between Dichodontium pellucidum and D. flavescens, supporting the status of separate species for the latter.

56. Systematic position of Dicranella palustris was revised by Stech [375] based on molecular data.

57. According to Ignatov (unpubl.) Dicranella heteromalla var. curvipes from Sakhalin and Kuriles is markedly different from $D$. heteromalla and has to be segregated as a separate species.

58. Records of Dicranoweisia cirrata from the Northern Russia and Urals [e.g. 150] seem to be erroneous: this species occurs in Russia only in Caucasus at Black Sea coast, on Juniperus and Curpessus [cf. 232].

59. Dicranum orientale from Siberia was described by Otnyukova [320] one year later than $D$. dispersum from Europe; these species were synonymized by Otnyukova \& Ochyra [326].

60. Dicranum tundrae has been synonymized with $D$. elongatum by Savicz-Lyubitskaya \& Smirnova [361], however Ignatova [224] found that this taxon may need further studies. Dicranum atratum Geh. was referred to D. elongatum by Savicz-Lyubitskaya \& Smirnova [361].

61. Records of East Asian species Dicranum hamulosum and Oncophorus crispifolius from Kazakhstan [356] seem to be doubtful.

62. Many previous records of Dicranum muehlenbeckii belong to $D$. brevifolium, $D$. dispersum, etc., and the real range of this species is obviously more limited than those found from literature records.

63. The status of Dicranum spadiceum Zett. var. subscabrifolium Schljak. is probably higher than a variety [224], however its distribution is not well known; it occurs at least on Kola Peninsula, in Caucasus, Urals and South Siberia.

64. Records of Dicranum spurium from N-UR [150, 230] were found to be erroneous [207].
65. According to Virchenko (unpubl.), a record of Dicranum viride from Crimea [66] belongs in fact to D. tauricum.

66. There is a certain inconsistency in the circumscription of Didymodon acutus and D. icmadophilus between different authors, thus their distribution needs a major revision; many previous records of $D$. acutus were not confirmed [e.g. 207].

67. Schljakov [363] considered Didymodon gorodkovii as a species; the combination in the status of variety was published by Afonina: Didymodon asperifolius var. gorodkovii (Abramova \& I.I.Abramov) Afonina, Problemy Bryologii 13, 1989 (basionym: Didymodon rufus var. gorodkovii Abramova \& I. I. Abramov, Trudy Arkt. i Antark. Nauchn.-Issl. Inst. 224: 220. 1963). This combination was not included in supplements to Index Muscorum.

68. Didymodon incrassatus was synonymized with $D$. australasiae by Jiménez \& al. [243].

69. Numerous records of Didymodon vinealis from the Middle European Russia and Urals were found to be erroneous [207].

70. Large plants of Ditrichum flexicaule were treated by Frisvoll [171] in a separate species, D. crispatissimum; the earlier name for the latter being D. gracile; their differences were nicely illustrated recently by Lüth [276]; in Russia distribution of these taxa remains poorly known, partly due to them not being considered as good species [cf. 207].

71. Ignatov \& Ignatova [207] lectotypified the genus Drepanium with Drepanium (Stereodon) recurvatum.

72. The taxonomy of Encalypta rhaptocarpa-group remains not totally resolved; Horton [193] segregated the group of E. rhaptocarpa without a peristome, but left it without any formal name; some old publications referred these eperistomate plants to $E$. spathulata or E. trachymitria; Horton however demonstrated that the real E. spathulata is distinct, whereas Mogensen [301] showed that E. trachymitria has a peristome, although quite different from that of $E$. rhaptocarpa; thus at the moment eperistomate $E$. rhaptocarpa remains unnamed, and records of $E$. spathulata or E. trachymitria seem to belong mainly (if not totally) to eperistomate E. rhaptocarpa .

73. Horton [193] demonstrated that all Asian collections of Encalypta procera-streptocarpa complex belong to E. procera and suggested that E. streptocarpa does not occur in Asia; the occurrence of the latter species in Ural Mountains is also problematic, despite numerous records.

74. Asian collections of Encalypta vulgaris often intergradate with eperistomate E. rhaptocarpa and may represent just a variation of the latter species.

75. Entodon cladorrhizans was excluded from bryoflora of Russia by Ignatov \& al. [200]. 
76. Entodon sinense was synonymized with $E$. giraldii by Tan [378].

77. According to Abramov \& al. [10], all specimens reported from KAZ $[155,296]$ and TAD as Entosthodon attenuatus belong in fact to Funaria aequidens.

78. All records of Entosthodon fascicularis from Urals [cf. 150] seem to be erroneous [cf. also 207]; Abramov \& al. [10] also did not confirm the records from Middle Asia.

79. Taxonomy of Entosthodon (Funaria) muhlenbergiigroup of species has been revised by Nyholm \& Crundwell [125], and according to their approach all the records from Russia proved to be E. pulchellus; however numerous records are based on still unrevised collections.

80. Ephemerum minutissimum is treated by some authors as only an infraspecific unit of $E$. serratum and reported under the latter name without recognition of subspecies or varieties; for example, in Latvia the latter species was reported [e.g. 3] although all the records in fact belong to the former taxon [2].

81. Records of Eucladium verticillatum from South Siberia [e.g. 197, 70] were not confirmed by recent revision of Ignatova.

82. Records of Eurhynchium striatum from Siberia [cf. 70] obviously belong to E. angustirete, as it is true also for many old records from East Europe.

83. According to Cherdantseva (unpubl.), record of Fabronia matsumurae from Russian Far East [199] was based on the misidentification of $F$. ciliata.

84. Abramova \& Abramov [22] recorded Fabronia pusilla for Irkutsk Province, but no specimens are found in LE; more likely this is a misidentification of $F$. ciliaris.

85. Taxonomy and distribution of Fissidens arcticus was discussed by Czernyadjeva [134].

86. Bruggeman-Nannega [see 190] argued that Fissidens gymnandrus, F. incurvus, F. viridulus are good species; in Russia they were considered for a long time within the circumscription of $F$. bryoides s.l., thus data on their distribution are too fragmentary and obviously inadequate.

87. The occurrence of Fissidens gracilifolius in North Asia has to be confirmed.

88. Savicz-Lyubitskaya \& Smirnova [361] synonymized Fissidens karataviensis with $F$. grandifrons, however Mamatkulov [295, 296] accepted the former species.

89. According to Gorobets [178], all records of Fissidens osmundoides from Primorsky Territory [e.g. in 76] belong in fact to F. gymnogynus; however F. osmundoides occurs in Khabarovsk Territory [e.g. 221].

90. Fissidens pusillus-group badly needs revision: during its complicated taxonomic and nomenclatural history this species once was considered in broad sense, including what is called now F. gracilifolius, and maybe some records belong to that species.

91. According to Cherdantseva, records of Fontinalis dalecarlica from RUS-AS: S-FE are erroneous.

92. Report of Funaria microstoma from RUS-EUR:

NE, Komi Republic, is doubtful.

93. Grimmia mesopotamica was synonymized with $G$. capillata by Muñoz \& Pando [306].

94. Numerous records of Grimmia montana were erroneous due to misinterpretation of diagnostic characters of this species for example in Russia, this is a very rare species [234].

95. Grimmia muehlenbeckii was treated by some authors as a variety of G. trichophylla, and some publications reported G. trichophylla s.l., obscuring distribution of these two species; according to Muñoz \& Pando [306], all the records of G. trichophylla east of Poland belong in fact to G. muehlenbeckii; all the revised material from Russia agrees with this conclusion, but material from Baltic and Caucasian countries is still awaiting a revision.

96. Records of Grimmia ovalis from the Polar Ural $[150]$ and North Ural $[415,150]$ were not confirmed by Ignatova \& Muñoz [234].

97. Muñoz \& Pando [306] failed to find the type of Grimmia laevidens and did not comment it; Greven [180] included this species into the synonymy of $G$. sessitana.

98. Greven [180] considered Grimmia crassifolia as a species of its own, but Ignatova \& Muñoz [234] synonymized it with $G$. tergestina.

99. No correctly identified specimen of Grimmia trichophylla from Russia has been found during the revision of Ignatova \& Muñoz [234]; however no collections from Kaliningrad Province were studied, and material from other countries also was not in the focus of that study.

100. Records of Grimmia unicolor from the North Ural $[150,415]$ were not confirmed by Ignatova \& Muñoz [234].

101. Species of Gymnostomum (especially G. aeruginosum-G. calcareum) in Russia need total revision; descriptions in different publication treat species limits in different ways.

102. Paratypes of Gymnostomum boreale were cited in the original description as being from Finland, but currently their localities are situated within Russian Karelia.

103. The only record of Haplocladium angustifolium from Middle European Russia is from Zhiguli Reserve by Mordvinov [304]; additional confirmation is needed; a record of this species for Caucasus by Ignatov \& al. [212] is erroneous.

104. Ivanova \& al. [239] did not confirm the occurrence of Herzogiella turfaca in Arctic Yakutia.

105. Homalia japonica was referred to H. trichoma- 
noides by Iwatsuki [242]; in the monograph of the genus, He [181] considered it as a subspecies H. trichomanoides ssp. japonica (Besch.) S.He.

106. Homalia woronowii described from Adzharia was synonymized with $H$. webbiana by $\mathrm{He}$ [181]; the latter species is otherwise known only from Macaronesia.

107. Many authors include Hymenoloma compactum (Dicranoweisia compacta) into H. crispulum, as a synonym or variety; however it is considered as an independent species and recorded for UKR: C [66], RUSEUR: NW [361, 84], KAZ [356], RUS-AS: S-SIB [70].

108. Hyocomium armoricum was reported by Ignatov \& Afonina [199] based on the record of Düll [154], without any exact reference.

109. Hypnum densirameum was reported from RUSAS: S-FE [76], but Afonina reidentified the specimen as Stereodon fauriei.

110. All records of Hypnum imponens from Asian Russia are probably erroneous; Ivanova \& al. [239] excluded it from the moss flora of Yakutia.

111. Hypnum jutlandicum is close to H. cupressiforme, and sometimes is considered as its variety $H$. cupressiforme var. ericetorum Bruch et al.

112. Kruijer [259] revised the genus Hypopterygium, and synonymized $H$. japonicum with $H$. flavolimbatum.

113. Churchill [119] synonymized Jaffueliobryum latifolium with $J$. wrigthii, however Ignatov \& Cao Tong [202] considered J. latifolium as a species of its own.

114. Kindbergia praelonga (Eurhynchium praelongum) was reported from many areas, but, for example, in Russia no correct records other than in Caucasus were proved (Ignatov, unpubl.); however a record from Kaliningrad Province is likely to be correct.

115. Leptodontium styriacum was synonymized with L. flexifolium by Ignatov \& al. [201].

116. He [182] published a world revision of Leptopterigynandrum, recognizing three species in Russia and few more in neighboring regions of Mongolia and China; the record of Leptopterigynandrum austro-alpinum from Ural Mts. [416] was erroneous.

117. Records of Lescuraea mutabilis from Siberia and northern part of Urals are doubtful and probably belong to L. saxicola, which was included in L. mutabilis in some earlier taxonomic treatments.

118. Caucasian collections referred to Lindbergia brachyptera are treated here as a separate species, $L$. grandiretis (Ignatov \& Ignatova, unpubl.).

119. Meesia longiseta was erroneously reported from Russian part of Caucasus by Onipchenko \& Ignatova [318].

120. The circumscription of genera of Pottiaceae is given according to Zander [411]; the main difference from the previous check-list is the inclusion of most species referred to Desmatodon, Pottia and Phas- cum to the genus Tortula, although some species of these genera are transferred to Microbyum; also, Tortula ruralis and similar species are segregated into the genus Syntrichia.

121. Records of Mnium hornum from eastern parts of European Russia including Urals were reidentified [cf. 207]; Asian records are also doubtful, and plants from East Asia were referred to a separate species, Mnium orientale R.E. Wyatt, Odrzykoski \& T.J. Kop., that however still was not found in the Russian Far East.

122. Koponen [253a] synonymized M. magnirete with M. ambiguum. Later Koponen [253b] synonymized M. ambiguum and M. laevinerve with M. lycopodioides, but some authors, e.g. Czernyadjeva [140] thought that M. laevinerve is a good species.

123. Ignatov (unpubl.) found that the holotype of Pseudoleskea korjakorum belongs to Myrinia pulvinata.

124. Myurella tenerrima was reported from RUS-AS: S-FE [212], basing on Lazarenko' record for Kedrovaya Pad Reserve, which is likely an error; recent list of mosses of this reserve [174] does not include this species.

125. The only population of Myuroclada in Europe is a result of unintentional introduction in botanical garden of Rostov-na-Donu city [207, 404].

126. Records of Orthothecium rufescens from the Arctic [e.g. 47] and Siberia proved to be $O$. chryseon, cf. [200].

127. Records of Orthotrichum affine from Kamchatka are dubious [140], and all so-called collections from the southern Russian Far East were found to be $O$. sordidum (Cherdantseva, unpubl.).

128. Judging from the illustration in Eremina [155], Orthotrichum laevigatum from Kazakhstan belongs to $O$. iwatsukii; other records outside Arctic also likely belong to $O$. iwatsukii.

129. Orthotrichum sibiricum was synonymized with O. pallens by Lewinsky [270].

130. Orthotrichum stellatum was reported with a question mark by Ignatov \& Afonina [199] for RUS-AS: S-FE; however, no collections of this species are known.

131. Recent revision of Cherdantseva (unpubl.) revealed that all records of Oxyrrhynchium hians from southern Russian Far East belong to Bryhnia.

132. All records of Oxyrrhynchium schleicheri from Russia (except Caucasus and maybe also Kaliningrad Province) and Kazakhstan are doubtful (all checked specimens were reidentified).

133. Many old records of Oxyrrhynchium speciosum from Russia were reidentified.

134. Many authors considered Palustriella falcata as a variety or synonym of $P$. commutata, thus the distribution of the former species is not well known. 
135. Ignatov reported Philonotis mollis for Altai [117], but later Koponen (unpubl.) reidentified this material as $P$. falcata.

136. According to Cherdantseva (unpubl.), the record of Philonotis seriata from Kuril Islands [77] belongs to $P$. yezoana.

137. According to Koponen [252], Plagiomnium elatum and $P$. affine are absent in North Asia and all previous records were erroneous; the old species concept of $P$. affine included $P$. ellipticum, so older records may imply the latter species.

138. According to Cherdantseva (unpubl.), all records of Plagiomnium rostratum from Russian Far East are erroneous.

139. Record of Plagiomnium undulatum from Ural [150] seems to be erroneous.

140. Ukrainskaya [390] provided description and distribution map of Plagiothecium berggrenianum in Russia.

141. Plagiothecium cordifolium and P. platycladum reported from Russian Far East by Lazarenko [268] are little known species, more likely synonymous with some other species.

142. Records of Plagiothecium curvifolium from Russian Far East has to be confirmed since the Pacific population seems to be quite isolated from the European one.

143. Records of Plagiothecium neckeroideum in RUSAS (S-FE [76]) were based on the erroneous identifications of $P$. euryphyllum (Cherdantseva, unpubl).

144. According to Ukrainskaya [389] numerous records of Plagiothecium piliferum from Russia were based mainly on misidentifications of Isopterygiopsis muelleriana; she cited only one correctly identified specimen from Yakutia [389], but later confirmed some specimens from NW European Russia [cf. 207].

145. We think that Plagiothecium succulentum is not a good species and must be synonymized either with $P$. cavifolium, or with $P$. nemorale.

146. Records of Pleuridium acuminatum from Russian Far East [e.g. 361] were obviously based on the confusions in its synonymy with $P$. subulatum; Matsui \& Iwatsuki [298] synonymized $P$. acuminatum with P. subulatum, but Europeaen [e.g. 190] authors usually keep them separate.

147. Pogonatum dentatum is rapidly spreading in East Europe, where it was known before only in mountain and arctic regions (cf. Hyvonen [195]).

148. Records of Pogonatum inflexum from Georgia belong to $P$. neesii [56].

149. Records of $P$. leucostoma ( $=P$. gracillima) from Primorsky Territory (RUS-AS: S-FE [76]), according to revision of Czernyadjeva [131], belong to $P$. proligera; East Siberian plants were not revised by her, but likely also belong to P. proligera.

150. Pohlia torrentium is usually not separated from $P$. filum, although some authors suggest recognizing it as a species $[364,40]$.

151. Polytrichum commune var. perigoniale (Michx.) Hampe, or P. perigoniale Michx., is sometimes considered as a distinct taxon, but more commonly is not separated from P. commune; its distribution can not be well understood based on the relatively scarce available records; Hill \& al. [190] do not accept this variety and $P$. commune var. humilis Sw., but accepted Polytrichum uliginosum (Wallr.) Schriebl ( $=P$. commune var. uliginosum Wallr.); the latter taxon was reported in Russia by Savicz-Lyubitskaya \& Smirnova [361], who mentioned its distribution as not different from $P$. commune; further confirmation however is needed for separating this species from P. commune.

152. According to Fedosov \& Ignatova [165], all Siberian records of Pseudocrossidium hornschuchianum and all Russian records of $P$. revolutum were based on erroneously identified specimens.

153. Leskeella incrassata was described from GEO [102] and subsequently reported in ARM [297] and AZE [277]; however, the distinction between P. nervosa and $L$. incrassata seems to be unstable: bistratose lamina, the main diagnostic character of the species, occurs sporadically in southern population of the latter species and if this character is accepted as of taxonomic importance, then the distribution of these two taxa must be totally reconsidered.

154. All checked collections of Psilopilum from nonArctic regions of South and Eastern Siberia were found to belong to Oligotrichum falcatum [238].

155. Pisarenko [337] found that on salty soils in South Siberia and Kazakhstan plants occur with phenotypes transitional between Pterygoneurum kozlovii and $P$. subsessile.

156. Oesau [317] suggested that plants from the SE European Russia belong to P. papillosum Oesau that was recently described from the Central Europe; Russian material has to be revised to understand the status and distribution of this taxon.

157. Arikawa [64] demonstrated by molecular phylogenetic methods that plants of "Pylaisia polyantha" from the Far East of Russia markedly different from the European one; he referred the former to P. curviramea, a species described from Central China.

158. According to Arikawa [64], Pylaisia intricata occurs only in North America, and Asian plants commonly called $P$. intricata belong to $P$. stereodontoides.

159. Pylaisiadelpha yokohamae (Broth.) W.R.Buck was considered as a synonym of $P$. tenuirostris by Abramova \& Abramov [33] and Iwatsuki [241], but in more recent papers by Tan \& Jia Yu [380] and Iwatsuki [242] these species are recognized as distinct; Russian material may belong to both species, but critical revision 
is needed; Clastobryella kusatsuensis (Besch.) Z.Iwats. reported from RUS-AS (S-FE [76, 77]) is a synonym of Pylaisiadelpha yokohamae [242].

160. According to Koponen \& Afonina [253c] Rhizomnium punctatum is absent in Far East of Russia; Ivanova \& al. [239] excluded it from the moss flora of Yakutia.

161. According to Gorobets [178], all records of Rhodobryum roseum from Primorsky Territory [e.g. 76] belong to $R$. ontariense; however the former species occurs in Khabarovsk Territory [e.g. 221]

162. The record of Rhynchostegium confertum in Russian Far East [268] seems doubtful, no record of this species east of Caucasus has been confirmed by Ignatov (unpubl.)

163. Huttunen \& al. [194] demonstrated that European and Asian plants, commonly referred to Rhynchostegium (Platyhypnidium) riparioides belong to two rather unrelated species; the plants from Siberia and Far East maybe have to be called Rhynchostegium muelleri A. Jaeger.

164. Ignatov \& Ignatova [207] segregated species of Rhytidiadelphus squarrosus-group as a new genus Rhytidiastrum; however they thought that the type genus of Rhytidiadelphus is R. triquetrus, omitting earlier lectotypification by Grout, who lectotypified the genus with $R$. squarrosus; before the nomenclature of Rhytidiadelphus s.l. is finally fixed we prefer to accept this genus in the traditional circumscription.

165. Recently Hedenäs [188] found that monoicous and dioicous species of Warnstorfia were well separated in phylogenetic analysis, and thus he suggested all dioicous species should be placed in the genus Sarmentypnum.

166. Many records of Schistidium apocarpum were based on the broad concept of this species $S$. apocarpum s. 1., which includes about 40 «narrow» species in East Europe and North Asia.

167. Recent revision of Ignatova did not confirm the presence of Schistidium confertum in Russia, although it possibly occurs in Caucasus.

168. Many records of Schistidium strictum were based on the previous broad concept of this species. However according to Blom [97], S. strictum does not occur on the territory of the present check-list; most previous records of this species should be referred now to $S$. papillosum and $S$. boreale.

169. Eurhynchium altaicum Ignatov was described from a single plant [198]; new collections from the type locality allow us to understand its position in the genus Scuiro-hypnum (Ignatov, unpubl.).

170. Record of Sciuro-hypnum latifolium from Caucasus [212] was erroneous.

171. Distribution and taxonomy of Scouleria in Russia is discussed by Kurbatova [260].

172. Siberian records of Seligeria diversifolia have to be confirmed; some of so-named collections from this region were reidentified as S. campylopoda. The record from South Urals was erroneous [cf. 302].

173. Mogensen \& Goldberg [302] reidentified specimen from Vishera Reserve (N-UR) as S. galinae, therefore the record of $S$. donniana in [212] is erroneous.

174. Partial revision of collections of Seligeria polaris from South Siberia revealed that some of them belong to other species; currently this species is known only from Arctic and Subarctic regions.

175. The occurrence of Seligeria trifaria in Siberia should be confirmed; partial revision revealed that some of socalled collections belong to $S$. tristichoides.

176. Corley \& al. [121] synonymized Sphagnum jensenii with $S$. annulatum, and some authors followed this suggestion and reported $S$. annulatum from certain areas of Russia; Flatberg [169] considered these species as distinct; according to Maksimov (unpubl.) the S. annulatum s.str. is definitely known only from NW European Russia.

177. According to Abolina (pers. com.) Latvian plants of Sphagnum imbricatum complex belong to $S$. austinii.

178. Semenov [365] described four species of Sphagnum from Altai: S. elenkinii, gordjaginii, krylovii, vereschaginii; Savicz [357] heavily criticized quite incomplete descriptions, suggesting simply to ignore these species. However these descriptions are valid, and the search of types is necessary to elucidate their identity.

179. Previous broad concept of Sphagnum imbricatum included numerous records from other regions: LTV [244], LAT [3], BEL [58], RUS-EUR (ARC [47], NW[407], KLN [310]), GEO [117], RUS-AS (ARCWSIB [136], W-SIB [265], ARC-YAK [47, 239]); here however we follow the narrow concept of Flatberg $[168,190]$, who left in $S$. imbricatum only East Asian plants, referring European species to $S$. austinii and S. affine, and arctic species to $S$. steerei; additional study is needed however to understand identity of e. g. West Siberian plants.

180. Ignatov \& Ignatova [207] lectotypified the genus Stereodon with Stereodon callichrous (Brid.) Braithw. (=Hypnum callichroum Brid.).

181. According to revision of Afonina [41], in Russia Stereodon (Hypnum) callichrous occurs only in Europe, and some specimens from Far East possibly belong to Hypnum callichroum var. japonicum Ando, while many other records were based on Stereodon holmenii.

182. Accoding to Ignatov \& al. [200], all Asian collections cited as Hypnum fertile belong in fact to Stereodon fauriei.

183. Stereodon holmenii has been discovered in many parts of Russia in the course of recent revision by Afonina [45].

184. Ivanova \& al. [239] excluded Stereodon palle- 
scens from Yakutia, where it was reported previously [e. g. 212, 47].

185. The combination Stereodon procerrimus (Molendo) Bauer, Musci Eur. Exsic., ser. 34: 6, No 1694. 1924, based on Hypnum procerrimum Molendo, Flora 49: 458. 1866 was not included in Index Muscorum and in Tropicos database.

186. Records of Stereodon (Hypnum) tristo-viride from RUS-AS (S-FE) in Ignatov \& Afonina [199] were based on misidentifications.

187. Tan \& al. [379] found that Cephalocladium zerovii described from South Siberia belongs to the genus Struckia; however the identity of the Siberian Struckia was resolved differently [cf. 216].

188. There is no agreement on the status of Syntrichia calcicola among recent authors [cf. 173 and 394].

189. Syntrichia pagorum was synonymized with S. laevipila by Gallego [173].

190. According to Bachurina \& Melnichuk [66], Tetrodontium ovatum is not different from $T$. repandum, although Hill \& al. [190] keep them as separate species.

191. Generic concepts in Thuidiaceae are mainly according to Touw [383].

192. Touw [383] put Thuidium philibertii into synonymy of $T$. assimile.

193. Records of Thuidium tamariscinum from N-UR, S-UR [150] were not confirmed [207].

194. Record of Timmia megapolitana from CAUC [212] was erroneous, excluded in Ignatova $\&$ al. [231].

195. According to the original description, Timmiella corniculata does not differ from $T$. barbuloides in the main diagnostic characters; type material is absent in $\mathrm{LE}$ and $\mathrm{H}$; the very scanty material in $\mathrm{S}$ lacks sporophytes that are essential for the identification Timmiella species; judging from the description and also from the fact that $T$. barbuloides is quite common in Pacific North-West of North America we preliminary include $T$. corniculata in T. barbuloides.

196. Tortella brotheri is a neglected taxon whose real identity needs further studies.

197. Corley \& al. [121] synonymized Tortula fiori with T. revolvens, however Abramov \& al. [11] keep them as separate species.

198. Mamatkulov validated combination Semibarbula trachyphylla (Broth.) Laz. ex Mamatkulov, F1. Bryoph. Tadjik SSR 1: 186. 1990, that was suggested by Lazarenko without citing of basyonym.

199. Trichostomum brachydontium was reported for Russian Far East by Podpera [340]; Bardunov \& Cherdantseva [76] considered this record as doubtful.

200. Tuerckheimia svihlae was erroneously reported by Ignatov for Altai [198].

201. Records of Zygodon conoideus from Siberia and Far East were based on Z. sibiricus, a recently described species that differs from $Z$. rupestris in presence of a peristome [210]; thus the presence of the latter species in Russia in the regions other than Caucasus and Karelia are questionalble; however some authors thought that this difference is rather insufficient and further studies may show identity of $Z$. sibiricus, and Z. rupestris [410].

202. Zygodon viridissimus was often recorded sensu lato, including $Z$. rupestris and $Z$. sibiricus, thus their distribution has to be revised; $Z$. viridissimus from Carpathians [66] has been referred by Virchenko [403] to Z. rupestris and Z. dentatus.

\section{SYNONYMS}

Abietinella abietina var. hystricosa (Mitt.) Sakurai $=$ Abietinella abietina

- hystricosa (Mitt.) Broth. = Abietinella abietina

Acrocladium cuspidatum (Hedw.) Lindb. = Calliergonella cuspidata

Aloina ericaefolia Kindb. = Aloina ambigua

Amblystegiella confervoides (Brid.) Loeske = Serpoleskea confervoides

- jungermannioides (Brid.) Giacom. = Platydictya jungermannioides

- sprucei (Bruch) Loeske = Platydictya jungermannioides

- subtilis (Hedw.) Loeske = Serpoleskea subtilis

Amblystegium compactum (Drumm. ex Müll.Hal.) Austin $=$ Conardia compacta

- confervoides (Brid.) Bruch et al. = Serpoleskea confervoides

- curvipes Schimp. = Hygroamblystegium humile

- fluviatile (Hedw.) Bruch et al. = Hygroamblystegium fluviatile

- humile (P.Beauv.) Crundw. = Hygroamblystegium humile

- jungermannioides (Brid.) A.J.E.Sm. = Platydictya jungermannioides

- juratzkanum Schimp. = Amblystegium serpens var. juratzkanum

- kochii Schimp.= Hygroamblystegium humile

- palustre (Brid.) Lindb. = Hygrohypnum luridum

- rigescens Limpr. = Amblystegium serpens

- riparium (Hedw.) Bruch et al. = Leptodictyum riparium

- saxatile Schimp. = Amblystegium radicale

- sprucei (Bruch) Bruch et al.=Platydictya jungermannioides

- subtile (Hedw.) Bruch et al. = Serpoleskea subtilis

- tenax (Hedw.) C.E.O.Jensen = Hygroamblystegium tenax

- varium (Hedw.) Lindb. = Hygroamblystegium varium

Andreaea amurensis Broth. = Andreaea rupestris

- assimilis Müll.Hal. = Andreaea rupestris var. papillosa

- compacta Müll.Hal. = Andreaea rupestris var. papillosa 
- cuspidata Müll.Hal. = Andreaea rupestris var. papillosa

- fauriei Besch. = Andreaea alpestris

- filiformis Müll.Hal. = Andreaea alpestris

- hartmanii Thed. = Andreaea obovata

- krauseana Müll.Hal. = Andreaea obovata

- papillosa Lindb. = Andreaea rupestris var. papillosa

- patens Müll.Hal. = Andreaea rupestris var. papillosa

- petrophila Ehrh. ex Fürnr. = Andreaea rupestris

- planinervis Lindb. ex G.Roth = Andreaea heinemannii

Anisothecium crispum (Hedw.) C.E.O.Jensen = Dicranella crispa

- grevilleanum (Brid.) Arnell \& C.E.O.Jensen $=D i$ cranella grevilleana

- humile (R.Ruthe) Lindb. = Dicranella humilis

- palustre (Dicks.) I.Hagen = Dichodontium palustre

- rigidulum (Hedw.) C.E.O.Jensen = Dicranella humilis

- rubrum Lindb. $=$ Dicranella varia

- rufescens (Dicks.) Lindb. = Dicranella rufescens

- schreberianum (Hedw.) Dixon=Dicranella schreberiana

- staphylinum (H.Whitehouse) Sipman, Rubers \& Riemann = Dicranella staphylina

- vaginale (Dicks.) Loeske = Dicranella crispa

- varium (Hedw.) Mitt. = Dicranella varia

Anoectangium amurense Broth. = Anoectangium thomsonii

- contortum Broth. = Anoectangium thomsonii

- schliephackei (Limpr. ex Schlieph.) Paris = Molendoa schliephackei

- sendtnerianum Bruch et al. = Molendoa sendtneriana

- tenuinerve (Limpr.) Paris $=$ Molendoa tenuinervis

Anomobryum concinnatum (Spruce) Lindb. = Anomobryum julaceum var. concinnatum

Anomodon apiculatus Sull. = Anomodon rugelii

- flagelliformis (L.I.Savicz) Granzow $=$ Haplohymenium flagelliforme

- longinervis Broth. = Haplohymenium longinerve

- minor subsp. integerrimus (Mitt.) Z.Iwats. = Anomodon minor

- rostratus (Hedw.) Schimp. = Claopodium rostratum

— tristis (Ces.) Sull. \& Lesq. = Haplohymenium triste

Aongstroemia fuji-alpina (Takaki) Z.Iwats. = Aongstroemia julacea

Aphanorrhegma patens (Hedw.) Lindb. = Physcomitrella patens

Astomum crispum (Hedw.) Hampe = Weissia longifolia

- crispum var. philibertii (Husn.) Wijk \& Margad. = Weissia levieri

- levieri Limpr. = Weissia levieri

Atrichum anomalum Milde = Polytrichastrum longisetum

- haussknechtii Jur. \& Milde = Atrichum flavisetum

- laevifolium (Lindb. \& Arnell) Paris = Atrichum flavisetum

- undulatum var. gracilisetum Besch. = Atrichum flavisetum

- undulatum var. haussknechtii (Jur. \& Milde) Frye = Atrichum flavisetum

Aulacomnium palustre var. imbricatum Bruch et al. =Aulacomnium palustre

Barbula acuta (Brid.) Brid. = Didymodon acutus

- acuta var. icmadophila (Schimp. ex Müll.Hal.) H.A.Crum $=$ Didymodon icmadophilus
- andreaeoides Kindb. = Didymodon subandreaeoides

- asperifolia Mitt. = Didymodon asperifolius

- cordata (Jur.) Loeske = Didymodon cordatus

- cylindrica (Taylor) Schimp. = Didymodon insulanus

- decurrens Laz. = Didymodon tophaceus

- ehrenbergii (Lorenz) M.Fleisch. = Didymodon tophaceus

- fallax Hedw. = Didymodon fallax

- fallax var. reflexa (Brid.) Brid. = Didymodon ferrugineus

- ferruginea Schimp. ex Besch. = Didymodon ferrugineus

- ferruginascens Stirt. = Bryoerythrophyllum ferruginascens

— gigantea Funck = Didymodon giganteus

- gracilis Schwägr. = Didymodon acutus

- hornschuchiana Schultz = Pseudocrossidium hornschuchianum

— icmadophila Schimp. ex Müll.Hal. = Didymodon icmadophilus

- incrassata Lindb. ex Broth. = Didymodon australasiae

- johansenii R.S.Williams = Didymodon johansenii

- lurida Hornsch. = Didymodon luridus

- obtusifolia Schwägr. = Tortula obtusifolia

- perobtusa (Broth.) P.C.Chen = Didymodon perobtusus

- recurvifolia Schimp. = Didymodon ferrugineus

- recurvirostris (Hedw.) Dixon $=$ Bryoerythrophyllum recurvirostrum

- reflexa (Brid.) Brid. = Didymodon ferrugineus

- reflexa var. robusta Braithw. = Didymodon maximus

- revoluta Brid. $=$ Pseudocrossidium revolutum

- rigidula (Hedw.) Milde $=$ Didymodon rigidulus

- rotundata Lindb. \& Arnell = Bryoerythrophyllum rotundatum

- rubella (Huebener) Mitt. = Bryoerythrophyllum recurvirostrum

- sinuosa (Mitt.) Grav. = Didymodon sinuosus

- spadicea (Mitt.) Braithw. = Didymodon spadiceus

- tophacea (Brid.) Mitt. = Didymodon tophaceus

— trifaria (Hedw.) Mitt. = Didymodon luridus

- vinealis Brid. = Didymodon vinealis

- vinealis var. cylindrica (Taylor) Boulay = Didymodon insulanus

- vinealis var. flaccida Bruch et al. = Didymodon insulanus

Bartramia crispa Brid. = Bartramia pomiformis

- deciduaefolia Broth. \& A.Yasuda=Bartramia ithyphylla

- ithyphylla var. strigosa (Wahlenb.) Hartm. = Bartramia ithyphylla

- pomiformis var. crispa (Brid.) Bruch et al. = Bartramia pomiformis

Blindia seligerioides Lindb. ex Broth. = Blindia acuta

Brachythecium abakanense Kaal. = Brachythecium buchananii

— asperrimum (Mitt. ex Müll.Hal.) Sull. = Brachythecium turgidum

- bryhnii (Kaurin) Kindb. = Brachytheciastrum collinum

- collinum (Schleich. ex Müll.Hal.) Bruch et al. = Brachytheciastrum collinum

- curtum (Lindb.) Limpr. = Sciuro-hypnum oedipodium

- dovrense (Limpr.) J.J.Amann = Sciuro-hypnum glaciale var. dovrense 
- eustegium Besch. = Eurhynchiadelphus eustegius

- fendleri auct. = Brachytheciastrum collinum

- glaciale Bruch et al. = Sciuro-hypnum glaciale

- glaciale var. dovrense Limpr. = Sciuro-hypnum glaciale var. dovrense

- groenlandicum (C.E.O.Jensen) Schljakov = Brachythecium coruscum

— jeniseense (Lindb. \& Arnell) Paris = Sciuro-hypnum starkei

- latifollium Kindb. = Sciuro-hypnum latifolium

- mildeanum var. udum (I.Hagen) Mönk. = Brachythecium udum

- oedipodium (Mitt.) A.Jaeger = Sciuro-hypnum oedipodium

- olympicum Jur. = Brachytheciastrum olympicum

- ornellanum (Molendo) Venturi \& Bott. = Sciuro-hypnum ornellanum

- oxycladum auct. non (Brid.) A.Jaeger $=$ Brachythecium laetum

- plumosum (Hedw.) Bruch et al.=Sciuro-hypnum plumosum

_ populeum (Hedw.) Bruch et al. = Sciuro-hypnum populeum

- reflexum (Starke) Bruch et al. = Sciuro-hypnum reflexum

- rotaeanum De Not. = Brachythecium capillaceum

- salebrosum subsp. rotaeanum (De Not.) J.J.Amann = Brachythecium capillaceum

- salebrosum subsp. turgidum (Hartm.) Hartm. = Brachythecium turgidum

- salebrosum var. capillaceum (F.Weber \& D.Mohr) Lorentz $=$ Brachythecium capillaceum

- salicinum Bruch et al. = Brachytheciastrum velutinum

- starkei (Brid.) Bruch et al. = Sciuro-hypnum starkei

- starkei var. curtum (Lindb.) Warnst. = Sciuro-hypnum oedipodium

— starkei var. explanatum auct. non (Brid.) Mönk. $=S c i$ uro-hypnum oedipodium

- thedenii Bruch et al. = Brachythecium erythrorrhizon

- trachypodium (Brid.) Bruch et al. = Brachytheciastrum trachypodium

- uncinifolium Broth. \& Paris = Sciuro-hypnum uncinifolium

- uralense Gorodkov = Lescuraea secunda

- velutinum (Hedw.) Bruch et al. = Brachytheciastrum velutinum

- wichurae (Broth.) Paris = Brachythecium garovaglioides Müll.Hal.

Breidleria arcuata (Molendo) Loeske $=$ Calliergonella lindbergii

Brotherella tenuirostris (Sull.) Broth. = Pylaisiadelpha tenuirostris $-\{159\}$

Brotherella yokohamae (Broth.) Broth. = Pylaisiadelpha tenuirostris $-\{159\}$

Bryhnia brachycladula Cardot = Bryhnia novae-angliae

- noesica (Besch.) Broth. = Bryhnia novae-angliae

Bryobrittonia pellucida R.S.Williams = Bryobrittonia longipes

Bryochenea sachalinensis (Lindb.) Gao \& Chang $=$ Echinophyllum sachalinense
Bryoerythrophyllum recurvirostrum var. dentatum (Schimp.) H.A.Crum, Steere \& L.E.Anderson $=B$. alpigenum

Bryohaplocladium angustifolium (Hampe \& Müll.Hal.) R.Watan. \& Z.Iwats. = Haplocladium angustifolium

- microphyllum (Hedw.) R.Watan. \& Z.Iwats. = Haplocladium microphyllum

- virginianum (Brid.) R.Watan. \& Z.Iwats. = Haplocladium virginianum

Bryoxiphium savatieri (Husn.) Mitt. = Bryoxiphium norvegicum var. japonicum

Bryum acutum Lindb. = Bryum axel-blyttii

- affine Lindb. \& Arnell = Bryum creberrimum

- algovicum fo. jailae (Sapjegin) L.I.Savicz = Bryum algovicum

- angustirete Kindb. = Bryum algovicum

- ardonense Breidl. = Bryum algovicum

- bicolor Dicks. = Bryum dichotomum

- blindii var. oblongum (Lindb.) Mönk. = Bryum oblongum

- blindii subsp.oblongum (Lindb.) Kindb. = Bryum oblongum

- caespiticium subsp. kunzei (Hornsch.) Podp. = Bryum kunzei

- caespiticium var. imbricatum Bruch et al. = Bryum kunzei

- caespiticium var. kunzei (Hornsch.) Braithw. = Bryum kunzei

- capillare var. elegans (Nees) Husn. = Bryum elegans

- capillare var. flaccidum (Brid.) Bruch et al. = Bryum moravicum

- cernuum (Hedw.) Bruch et al. = Bryum uliginosum

- cirrhatum Hoppe \& Hornsch. = Bryum lonchocaulon

- cirrhatum var. affine Podp. = Bryum creberrimum

- comense Schimp. = Bryum caespiticium

- crispulum Hampe = Bryum pseudotriquetrum

- cuspidatum (Bruch et al.) Schimp. = Bryum creberrimum

- demissum Hook. = Plagiobryum demissum

- demissum subsp. hultenii Ochi \& Perss. = Plagiobryum hultenii

- duvalii Voit $=$ Bryum weigelii

- erythrocarpum auct. = Bryum sect. Erythrocarpa

- flaccidum auct. = Bryum moravicum

— imbricatum auct. non (Schwägr.) Bruch et al. = Bryum amblyodon

- inclinatum (Sw. ex Brid.) Blandow = Bryum amblyodon

- kaernbachii Müll.Hal. = Bryum capillare

- lacustre (F.Weber \& D.Mohr) Blandow = Bryum knowltonii

- laetum Lindb. = Bryum oblongum

- laevifilum Syed = Bryum moravicum

- latifolium (Schwägr.) Brid. = Bryum schleicheri

- lisae var. cuspidatum (Bruch et al.) Margad. = Bryum creberrimum

- luridum R.Ruthe = Bryum arcticum

- mamillatum Lindb. = Bryum warneum

- microblastum Müll.Hal. = Bryum pallescens var. microblastum

- microerythrocarpum Müll.Hal. \& Kindb. ex Macoun = Bryum subapiculatum 
- obconicum Hornsch. ex Bruch et al. = Bryum pallescens

- obtusifolium Lindb. = Bryum cryophilum

- oelandicum H.Philib. = Bryum warneum

- pallens subsp. lundstroemii (Arnell) Podp. = Bryum pallens

- paradoxum H.Philib. = Bryum terskeiense

- pendulum (Hornsch.) Schimp. = Bryum algovicum

- planiusculum Lindb. \& Arnell = Bryum turbinatum

- pseudocrispulum (Podp.) L.I.Savicz, nom. illeg. = Bryum pseudotriquetrum

- pseudotriquetrum subsp. bimum (Schreb.) Hartm. = Bryum bimum

- purpurascens var. serotinum (Lindb.) C.E.O.Jensen = Bryum purpurascens

- saxatile I.Hagen = Bryum lonchocaulon

- schleicheri var. latifolium (Schwägr.) Schimp. = Bryum schleicheri

- serotinum Lindb. = Bryum purpurascens

- stirtonii Schimp. = Bryum elegans

- subelegans auct. non Kindb. = Bryum moravicum

- subglobosum Schlieph. = Bryum lonchocaulon

- subobtusifolium Müll.Hal. = Bryum cyclophyllum

- subrotundum Brid. = Bryum pallescens

- tortifolium Brid. = Bryum cryophilum

- turbinatum subsp. schleicheri (Schwägr.) Kindb. = Bryum schleicheri

- ventricosum Relh. = Bryum pseudotriquetrum

- ventricosum var. bimum (Schreb.) Hampe = Bryum bimum

- zieri Hedw. = Plagiobryum zieri

Buckiella undulata (Hedw.) Ireland = Plagiothecium undulatum

Buxbaumia indusiata Brid. = Buxbaumia viridis

Callialaria curvicaule (Jur.) Ochyra = Cratoneuron curvicaule

Calliergon cuspidatum (Hedw.) Kindb. = Calliergonella cuspidata

- richardsonii var. megalophyllum (Mikut.) Meyl. = C. megalophyllum

- richardsonii var. robustum (Lindb. \& Arnell) G.Roth = Calliergon megalophyllum

- sarmentosum (Wahlenb.) Kindb. = Warnstorfia sarmentosa

— stramineum (Dicks. ex Brid.) Kindb. = Straminergon stramineum

- trifarium (F.Weber \& D.Mohr) Kindb. = Pseudocalliergon trifarium

Camptothecium aureum (Spruce) Bruch et al. = Homalothecium aureum

- auriculatum (A.Jaeger) Broth. = Brachythecium auriculatum

- geheebii (Milde) Kindb. = Brachythecium geheebii

- lutescens (Hedw.) Bruch et al. = Homalothecium lutescens

- nitens (Hedw.) Schimp. = Tomentypnum nitens

- trichodes Lindb. = Tomentypnum nitens

Campyliadelphus polygamus (Bruch et al.) Kanda = Drepanocladus polygamus

— stellatus (Hedw.) Kanda = Campylium stellatum
Campylium adscendens (Lindb.) Perss. = Herzogiella adscendens

- arcticum (R.S.Williams) Broth. = Drepanocladus arcticus

- calcareum Crundw. \& Nyholm = Campylidium calcareum

- chrysophyllum (Brid.) Lange = Campyliadelphus chrysophyllus

- elodes (Lindb.) Kindb. = Campyliadelphus elodes

- halleri (Hedw.) Lindb. = Campylophyllum halleri

- hispidulum auct. non (Brid.) Mitt. = Campylidium [sommerfeltii \& calcareum]

- hispidulum (Brid.) Mitt. = Campylidium hispidulum

- hispidulum var. sommerfeltii (Myrin) Lindb. = Campylidium sommerfeltii

- krylovii (Podp.) Laz. = Podperaea krylovii

- polygamum (Bruch et al.) Lange \& C.E.O.Jensen $=$ Drepanocladus polygamus

- radicale (P.Beauv.) Grout $=$ Amblystegium radicale

- sommerfeltii (Myrin) Lange $=$ Campylidium sommerfeltii

- stellatum subsp. protensum (Brid.) C.E.O.Jensen = Campylium protensum

— stellatum var. protensum (Brid.) Bryhn = Campylium protensum

- zemliae (C.E.O.Jensen) C.E.O.Jensen $=$ Drepanocla dus arcticus

Campylophyllum calcareum (Crundw. \& Nyholm) Hedenäs $=$ Campylidium calcareum

- hispidulum auct. non (Brid.) Hedenäs = Campylidium [sommerfeltii \& calcareum]

— hispidulum (Brid.) Hedenäs = Campylidium hispidulum

- sommerfeltii (Myrin) Hedenäs = Campylidium sommerfeltii

Campylopus fragilis var. pyriformis (Schultz) Ångstr. = Campylopus pyriformis

- schwarzii Schimp. = Campylopus gracilis

- subulatus var. schimperi (Milde) Husn. = Campylopus schimperi

Catharinea $=$ Atrichum

Cephalocladium enerve (Broth.) Abramova \& I.I.Abramov = Struckia enervis

- zerovii Laz. = Struckia enervis

Ceratodon purpureus var. conicus (Hampe) Husn. = Ceratodon conicus

- purpureus var. rotundifolius Berggr. = Ceratodon heterophyllus

Cinclidium minutifolium Broth. = Cinclidium latifolium

Cinclidotus nigricans (Brid.) Wijk \& Marg. = Cinclidotus riparius

Cirriphyllum apiculigerum (Lindb. \& Arnell) Broth. = Sciuro-hypnum ornellanum

- cirrosum (Schwägr.) Grout = Brachythecium cirrosum

- ornellanum (Molendo) Loeske = Sciuro-hypnum ornellanum

- plumosum (Hedw.) Loeske \& M.Fleisch. = Sciurohypnum plumosum

- reichenbachianum (Huebener) Wijk \& Margad. = Sciuro-hypnum flotowianum

- tenuinerve (Lindb.) Wijk \& Margad. = Brachythecium tommasinii 
- tommasinii (Sendtn. ex Boulay) Grout = Brachythe cium tommasinii

- vaucheri Loeske \& M.Fleisch. = Brachythecium tommasinii

- velutinoides (Bruch et al.) Loeske \& M.Fleisch. = Sciuro-hypnum flotowianum

Clastobryella kusatsuensis (Besch.) Z.Iwats. = Pylaisiadelpha yokohamae - \{see annotation 159$\}$

Cratoneuron arcticum Steere $=$ Hygroamblystegium varium

- commutatum (Hedw.) G.Roth = Palustriella commutata

- commutatum var. falcatum (Brid.) Mönk. = Palustriella falcata

- decipiens (De Not.) Loeske = Palustriella decipiens

- falcatum (Brid.) G.Roth = Palustriella falcata

- filicinum var. atrovirens (Brid.) Ochyra = Cratoneuron filicinum

- filicinum var. curvicaule (Jur.) Mönk. = Cratoneuron curvicaule

Crossidium chloronotos auct. non (Brid.) Limpr. $=$ Crossidium crassinerve

- griseum (Jur.) Jur. = Crossidium squamiferum var. pottioideum

Ctenidium procerrimum (Molendo) Lindb. = Stereodon procerrimus

Cupressina ulophylla Müll.Hal. = Stereodon subimponens var. ulophyllum

Cynodontium alpestre (Wahlenb. ex Huebener) Milde = Cnestrum alpestre

- glaucescens (Lindb. \& Arnell) Paris = Cnestrum glaucescens

- polycarpon var. strumiferum (Hedw.) Schimp. $=C y$ nodontium strumiferum

Cyrto-hypnum minutulum (Hedw.) W.R.Buck \& H.A. Crum $=$ Pelekium minutulum

- pygmaeum (Bruch et al.) W.R.Buck \& H.A.Crum = Pelekium pygmaeum

- sparsifolium (Mitt.) W.R.Buck \& H.A.Crum = Pelekium versicolor

- vestitissimum (Besch.) W.R.Buck \& H.A.Crum = Bryochenea vestitissima

Desmatodon altipes Broth. = Tortula altipes

- arenaceus Sull. \& Lesq. = Tortula obtusifolia

- cernuus (Huebener ) Bruch et al.= Tortula cernua

- glacialis Funck ex Brid. = Tortula hoppeana

- heimii (Hedw.) Mitt. = Hennediella heimii

- heimii var. arctica (Lindb.) H.A.Crum = Hennediella heimii var. arctica

- latifolius (Hedw.) Brid. = Tortula hoppeana

- laureri (Schultz) Bruch et al. = Tortula laureri

- leucostoma (R.Br.) Berggr. = Tortula leucostoma

- meridionalis Luisier = Tortula marginata

- obtusifolius (Schwägr.) Schimp. = Tortula obtusifolia

- oxneri Laz. $=$ Tortula randii

- randii (Kenn.) Laz. $=$ Tortula randii

- suberectus (Drumm.) Limpr.= Tortula leucostoma

- systylius Schimp. = Tortula systylia

- ucrainicus Laz. = Tortula ucrainica

Dichodontium pellucidum var. flavescens (Dicks.) Moore = Dichodontium flavescens
Dicranella caucasica (Müll. Hal.) Broth. = Dicranella heteromalla

- heteromalla var. curvipes Lindb. = Dicranella curvipes

- palustris (Dicks.) Crundw. = Dichodontium palustre

- rigidula (Hedw.) Dix. = Dicranella humilis

- riparia (H.Lindb.) Mårtensson \& Nyholm = Kiaeria riparia

- schreberi Schimp. = Dicranella schreberiana

- schreberi var. grevilleana (Brid.) Mönk. = Dicranella grevilleana

- squarrosa (Schrad.) Schimp. = Dichodontium palustre

Dicranodontium longirostre (F.Weber \& D.Mohr) Bruch \& Schimp. $=$ Dicranodontium denudatum

Dicranoweisia crispula (Hedw.) Milde = Hymenoloma crispulum

- compacta (Schwägr.) Schimp. = Hymenoloma crispulum

— intermedia J.J.Amann = Hymenoloma intermedium

Dicranum affine Funck = Dicranum undulatum

- atratum Geh. = ?Dicranum elongatum

- bergeri Blandow = Dicranum undulatum

- congestum Brid. = Dicranum fuscescens

- congestum auct. non Brid. = Dicranum flexicaule

- elatum Lindb. = Dicranum drummondii

- fuscescens var. congestum (Brid.) Husn. = Dicranum flexicaule

- fuscescens var. flexicaule (Brid.) Wilson = Dicranum flexicaule

- muehlenbeckii var. brevifolium Lindb. = Dicranum brevifolium

- neglectum Jur. ex De Not. = Dicranum spadiceum

- orientale Otnyukova $=$ Dicranum dispersum

- robustum Blytt ex Bruch et al. = Dicranum drummondii

- rugosum Brid. = Dicranum polysetum

- spadiceum var. subscabrifolium Schljakov $=D i$ cranum spadiceum

- strictum Schleich. ex D.Mohr = Dicranum tauricum

- tundrae Lindb. \& Arnell = ?Dicranum elongatum

- undulatum auct. non Schrad. ex Brid. = Dicranum polysetum

Didymodon aaronis (Lorentz) J.Guerra = Didymodon australasiae

- fallax var. reflexus (Brid.) R.H.Zander = Didymodon ferrugineus

- fragilis Hook. \& Wilson = Tortella fragilis

- gorodkovii (Abramova \& I.I.Abramov) Schljakov = Didymodon asperifolius var. gorodkovii

- incrassatus (Lindb.) Broth. = Didymodon australasiae

- pusillus Hedw. = Ditrichum pusillum

- rigidulus subsp. andreaeoides (Limpr.) Wijk \& Margad. = Didymodon subandreaeoides

- rigidulus var. gracilis (Schleich. ex Hook. \& Grev.) R.H.Zander = Didymodon acutus

- rigidulus var. icmadophilus (Schimp. ex Müll.Hal.) R.H.Zander = Didymodon icmadophilus

- rubellus Bruch et al. = Bryoerythrophyllum recurvirostrum

- rufus Lorentz $=$ Didymodon asperifolius

— trifarius auct. non (Hedw.) Röhl = Didymodon luridus 
- vinealis var. luridus (Hornsch.) R.H.Zander $=$ Didy modon luridus

- vinealis var. flaccidus (Bruch et al.) R.H.Zander $=$ Didymodon insulanus

Diobelonella palustris (Dicks.) Ochyra = Dichodontium palustre

Distichium montanum I.Hagen = Distichium capillaceum

Ditrichum crispatissimum (Müll.Hal.) Paris = Ditrichum gracile

- flexicaule var. longifolium (J.E.Zetterst.) I.Hagen = Ditrichum gracile

- flexicaule var. sterile (De Not.) Limpr. = Ditrichum gracile

- giganteum R.S.Williams = Ditrichum gracile

- glaucescens (Müll.Hal.) Hampe = Saelania glaucescens

- heteromallum var. zonatum (Brid.) Podp. = Ditrichum zonatum

- homomallum (Hedw.) Hampe = Ditrichum heteromallum

- tenuifolium Lindb. = Ditrichum cylindricum

- tortile (Schrad.) Brockm. = Ditrichum pusillum

Dolichotheca $=$ Herzogiella

Drepanocladus aduncus var. kneiffii (Schimp.) Mönk. = Drepanocladus aduncus

- aduncus var. polycarpos (Blandow ex Voit) G.Roth = Drepanocladus aduncus

- badius (Hartm.) G.Roth = Loeskypnum badium

- brevifolius (Lindb.) Warnst. = Pseudocalliergon brevifolium

- capillifolius (Warnst.) Warnst. = Drepanocladus longifolius

- cossonii (Schimp.) Loeske = Scorpidium cossonii

- exannulatus (Bruch et al. ) Warnst. = Warnstorfia exannulata

- exannulatus var. tundrae (Arnell) Warnst. = Warnstorfia tundrae

- fluitans (Hedw.) Warnst. = Warnstorfia fluitans

- fluitans var. pseudostramineus (Müll.Hal.) Warnst. = Warnstorfia pseudostraminea

- h-schulzei (Limpr.) Loeske = Warnstorfia fluitans

- intermedius (Lindb.) Warnst. = Scorpidium cossonii

- kneiffii (Bruch et al.) Warnst. = Drepanocladus aduncus

- kurilensis Smirnova $=$ Warnstorfia fluitans

- lapponicus (Norrl.) Smirnova = Hamatocaulis lapponicus

— latifolius (Lindb. \& Arnell) Warnst. = Pseudocalliergon brevifolius

- lycopodioides (Brid.) Warnst. = Pseudocalliergon lycopodioides

- polycarpos (Blandow ex Voit) Warnst. = Drepanocladus aduncus

- procerus (Renauld \& Arnell) Warnst. = Warnstorfia procera

- pseudofluitans (Sanio) Warnst. = Drepanocladus aduncus

- pseudostramineus (Müll.Hal.) G.Roth = Warnstorfia pseudostraminea

- revolvens (Sw. ex anon.) Warnst. = Scorpidium revolvens
- revolvens var. intermedius (Lindb.) Wilson $=$ Scorpidium cossonii

- scorpioides (Hedw.) Warnst. = Scorpidium scorpioides

- simplicissimus Warnst. = Drepanocladus aduncus

- tenuinervis T. J.Kop. = Drepanocladus sordidus

- trichophyllus (Warnst.) Podp. = Warnstorfia trichophylla

- tundrae (Arnell) Loeske = Warnstorfia tundrae

- turgescens (T.Jensen) Broth. = Pseudocalliergon turgescens

- uncinatus (Hedw.) Warnst. = Sanionia uncinata

- vernicosus (Mitt.) Warnst. = Hamatocaulis vernicosus

- vernicosus var. lapponicus (Norrl.) G.Roth = Hamatocaulis lapponicus

Drummondia ussuriensis Broth. = Drummondia sinensis var. ussuriensis

Dryptodon atratus (Miel. ex Hornsch.) Limpr. = Grimmia atrata

Dryptodon patens (Hedw.) Brid. = Grimmia ramondii

Encalypta contorta Hoppe ex Lindb. = Encalypta streptocarpa

- rhabdocarpa Schwägr. = Encalypta rhaptocarpa

- rhaptocarpa var. leptodon Lindb. = Encalypta trachymitria

- rhaptocarpa var. spathulata (Müll. Hal.) Husn.= Encalypta spathulata

- vulgaris var. mutica Brid. = Encalypta mutica

Entodon cladorrhizans auct. non (Hedw.) Müll.Hal. = Entodon schleicheri

- compressus Müll.Hal. ex Cardot = Entodon challengeri

- orthocarpus (Brid.) Lindb. = Entodon concinnus

- rubicundus (Mitt.) A.Jaeger = Entodon flavescens

- sinense (Dixon) Laz. = Entodon giraldii

- sullivantii var. versicolor (Besch.) Mizushima $=$ Entodon sullivantii

Entosthodon pallescens Jur. = Entosthodon durieui

- subpallescens Laz. = Entosthodon angustifolius

Ephemerum serratum var. minutissimum (Lindb.) Grout = Ephemerum minutissimum

Erythrophyllum recurvirostre (Hedw.) Loeske $=$ Bryoerythrophyllum recurvirostrum

Eucladium aeruginosum (Sm.) C.E.O.Jensen = Gymnostomum aeruginosum

- recurvirostre (Hedw.) C.E.O.Jensen $=$ Hymenostylium recurvirostrum

- styriacum Glow. = Eucladium verticillatum

Eurhynchium altaicum Ignatov = Sciuro-hypnum altaicum

- circinatum (Brid.) Schimp. = Scorpiurium circinatum

- crassinervium (Taylor) Bruch et al. = Cirriphyllum crassinervium

- diversifolium Bruch et al. = Eurhynchiastrum pulchellum

- eustegium (Besch.) Dixon = Eurhynchiadelphus eustegius

- flotowianum (Sendtn.) Kartt. = Sciuro-hypnum flotowianum

- hians (Hedw.) Sande Lac. = Oxyrrhynchium hians

- meridionale (Bruch et al.) De Not. = Plasteurhynchium meridionale 
- praelongum (Hedw.) Bruch et al. = Kindbergia praelonga

- praelongum var. stokesii (Turner) Dixon $=$ Kindbergia praelonga

_ pulchellum (Hedw.) Jenn. = Eurhynchiastrum pulchellum

- pulchellum var. diversifolium (Bruch et al.) C.E.O. Jensen = Eurhynchiastrum pulchellum

- pulchellum var. praecox (Hedw.) Dixon = Eurhynchiastrum pulchellum

- pumilum (Wilson) Schimp. = Oxyrrhynchium pumilum

- riparioides (Hedw.) R.W.Richards = Rhynchostegium riparioides

- schleicheri (R.Hedw.) Milde = Oxyrrhynchium schleicheri

- speciosum (Brid.) Jur. = Oxyrrhynchium speciosum

- stokesii (Turner) Bruch et al. = Kindbergia praelonga

- striatulum (Spruce) Bruch et al.= Plasteurhynchium striatulum

- striatum var. pachycladum G.Roth = Eurhynchium angustirete

- striatum subsp. zetterstedtii (P.Størmer) Podp. $=$ Eurhynchium angustirete

- strigosum (F.Weber \& D.Mohr) Schimp. = Eurhynchiastrum pulchellum

- swartzii (Turner) Curn. = Oxyrrhynchium hians

- zetterstedtii P.Størmer = Eurhynchium angustirete

Fissidens adelphinus Besch. = Fissidens teysmannianus

- bambergeri auct. non Milde $=F$. crispus

- bryoides var. viridulus (Sw.) Broth. = Fissidens viridulus

- cristatus Wilson ex Mitt. = Fissidens dubius

- decipiens De Not. = Fissidens dubius

- gymnandrus Büse $=$ Fissidens bryoides var. gymnan drus

- incurvus var. tenuifolius (Boulay) A.J.E.Sm. = Fissidens gracilifolius

- japonicus Dozy \& Molk. $=$ F. nobilis

- julianus (Savi ex DC.) Schimp. = Fissidens fontanus

- limbatus Sull. = Fissidens crispus

- mildeanus Schimp. = Fissidens crassipes

- minutulus auct. non Wils. $=$ Fissidens gracilifolius

- obtusifolius auct. non Wils. = Fissidens arnoldii

— strictulus Müll.Hal. = Fissidens curvatus

- viridulus var. incurvus (Starke ex Röhl.) Waldh. = Fissidens incurvus

- viridulus var. tenuifolius (Boulay) A. J. E. Sm. =Fissidens gracilifolius

Fontinalis gracilis Lindb. = Fontinalis antipyretica var. gracilis

- mesopotamica Schiffn. = Fontinalis hypnoides var. duriaei

— nitida Lindb. \& Arnell = Fontinalis hypnoides var. duriaei

Funaria attenuata (Dicks.) Lindb. = Entosthodon attenuatus

- calcarea Wahlenb. = Entosthodon muhlenbergii

- dentata Crome $=$ Entosthodon muhlenbergii

- fascicularis (Hedw.) Lindb. = Entosthodon fascicularis
- handelii Schiffn. = Entosthodon handelii

- hibernica Hook. = Entosthodon muhlenbergii

- hungarica Boros = Entosthodon hungaricus

- hygrometrica var. arctica Berggr. = Funaria arctica

- mediterranea Lindb. = Entosthodon muhlenbergii

- muhlenbergii Turner $=$ Entosthodon muhlenbergii

- obtusifolia Weinm. = Funaria hygrometrica

- pallescens (Jur.) Lindb. = Entosthodon durieui

- pulchella H.Philib. = Entosthodon pulchellus

- subpallescens (Laz.) Smirnova = Entosthodon angustifolius

Geheebia gigantea (Funck) Boulay = Didymodon giganteus

Gollania amurensis Broth. = Gollania ruginosa

- densepinnata Dixon = Gollania turgens

- neckerella (Müll.Hal.) Broth. var. coreense (Cardot) Broth. = Gollania ruginosa

Grimmia affinis Hornsch. = Grimmia longirostris

- alpestris var. sessitana (De Not.) I.Hagen = Grimmia reflexidens

- alpicola auct. non Hedw. = Schistidium rivulare

- alpicola Hedw. = Schistidium agassizii

- andreaeopsis Müll.Hal. = Schistidium andreaeopsis

- apiculata Hornsch. = Grimmia fuscolutea

- apocarpa Hedw. = Schistidium apocarpum

- apocarpa var. gracilis Röhl. = Schistidium spp.

- apocarpa var. taimyrensis I.G.Borszcz. \& G.G.Borszcz. = Schistidium papillosum

- brotheri Lindb. ex Broth. = Grimmia hartmanii

- calvescens Kindb. = Grimmia funalis

- campestris Bruch ex Hook. = Grimmia laevigata

- cardotii Hérib. ex Seb. = Grimmia poecilostoma

- cavifolia Lindb. \& Arnell = Grimmia longirostris

- commutata Huebener = Grimmia ovalis

- crassifolia Lindb. ex Broth. = Grimmia tergestina

- curvata (Brid.) De Sloover = Grimmia ramondii

- dupretii Thér. = Schistidium dupretii

- gracilis Schleich. ex Schwägr. = Schistidium spp.

- hartmanii var. anomala (Hampe) Mönk. = Grimmia anomala

- hartmanii var. montenegrina Breidl. \& Szyszyl. = ?Grimmia hartmanii

- laevidens Broth. = Grimmia reflexidens

- maritima $\mathrm{Sm}$. ex R.Scott = Schistidium maritimum

- maritima var. pilifera I.Hagen = Schistidium maritimum subsp. piliferum

- mesopotamica Schiffn. = Grimmia capillata

- olympica E.Britton = Brachydontium olympicum

- ovalis auct. non (Hedw.) Lindb. = Grimmia longirostris

- ovata auct. non F.Weber \& D.Mohr = Grimmia ovalis

- patens (Hedw.) Bruch et al. = Grimmia ramondii

- pitardii Corb. $=$ Campylostelium pitardii

- pulvinata var. africana (Hedw.) Hook.f. \& Wilson = Grimmia pulvinata

- rivularis Brid. $=$ Schistidium rivulare

- sessitana De Not. = Grimmia reflexidens

- stricta Turner $=$ Schistidium spp.

- subsulcata Limpr. = Grimmia reflexidens

- tergestina var. poecilostoma (Cardot \& Sebille)

Loeske $=$ Grimmia poecilostoma 
- tergestina var. tergestinoides (Culm.) Podp. = ?Grimmia tergestina

- tergestinoides Culm. = Grimmia tergestina

- trichophylla var. muehlenbeckii (Schimp.) Husn. = Grimmia muehlenbeckii

- trichophylla var. septentrionalis Schimp. = Grimmia muehlenbeckii

- trichophylla var. tenuis (Wahlenb.) Wijk \& Margad. = Grimmia muehlenbeckii

Gymnostomum recurvirostrum Hedw. = Hymenostylium recurvirostrum

Habrodon leucotrichus (Mitt.) Perss. = Iwatsukiella leucotricha

Hedwigia ciliata var. leucophaea Bruch et al. = Hedwigia ciliata

Helicodontium pulvinatum (Wahlenb.) Lindb. = Myrinia pulvinata

- rotundifolium Arnell = Myrinia rotundifolia

Helodium lanatum (Brid.) Broth. = Helodium blandowii

- sachalinense (Lindb.) Broth. = Echinophyllum sachalinense

Heterocladium leucotrichum Mitt. = Iwatsukiella leucotricha

- papillosum (Lindb.) Lindb. = Pseudoleskeella papillosa

- squarrosulum Lindb. = Heterocladium dimorphum

Heterophyllium adscendens (Lindb.) Broth. = Herzogiella adscendens

- haldanianum (Grev.) M.Fleisch. = Callicladium haldanianum

- nemorosum (Koch ex Brid.) Kindb. = Heterophyllium affine

Homalia besseri Lobarz. $=$ Neckera besseri

- japonica Besch. = Homalia trichomanoides

- woronowii Thér = Homalia webbiana

Homaliadelphus laevidentatus (S.Okamura) Z.Iwats. = Homaliadelphus targionianus var. laevidentatus

Homalothecium aristatum Laz. = Homalothecium philippeanum

- geheebii (Milde) Wigh = Brachythecium geheebii

- nitens (Hedw.) H.Rob. = Tomentypnum nitens

- tokiadense (Mitt.) Besch. = Homalothecium laevisetum

Hondaella brachytheciella (Broth. \& Paris) Ando = Hondaella caperata

Hydrogrimmia mollis (Bruch et al.) Loeske = Grimmia mollis

Hygroamblystegium fallax (Brid.) Loeske = Cratoneuron filicinum

— irriguum (Hook. \& Wilson) Loeske = Hygroamblys tegium tenax

- tenax var. spinifolium (Schimp.) Jenn. = Hygroamblystegium tenax

Hygrohypnella duriuscula (De Not.) Ignatov \& Ignatova = Ochyraea duriuscula

Hygrohypnum alpestre (Hedw.) Loeske = Ochyraea alpestris

- bestii (Renauld \& Brynh) Broth. = Hygrohypnella bestii

- cochlearifolium (Venturi) Broth. = Ochyraea cochlearifolia
- dilatatum auct. non (Wilson) Loeske $=$ Ochyraea $d u-$ riuscula

- duriusculum (De Not.) D.W.Jamieson = Ochyraea duriuscula

- ehlei (Arnell) Broth. = Hygrohypnella polare

- eugyrium (Bruch et al.) Broth. = Pseudohygrohypnum eugyrium

- molle (Hedw.) Loeske = Ochyraea mollis

- molle subsp. dilatatum (Wilson) Grout = Ochyraea duriuscula

- norvegicum (Bruch et al.) J.J.Amann = Ochyraea norvegica

- ochraceum (Turner ex Wilson) Loeske = Hygrohyp nella ochracea

- palustre Loeske = Hygrohypnum luridum

- polare $($ Lindb.) Loeske $=$ Hygrohypnella polare

- polare var. falcatum (Bryhn) Broth. = Hygrohypnella polare

- rivulare Broth. = Ochyraea alpestris

- smithii (Sw.) Broth. = Ochyraea smithii

- smithii var. cochlearifolium (Venturi ex De Not.) Mönk. = Ochyraea cochlearifolia

- subeugyrium (Renauld \& Cardot) Broth. = Pseudohygrohypnum subeugyrium

Hylocomium alaskanum (Lesq. \& James) Austin $=$ Hylocomium splendens var. obtusifolium

- brevirostre (Brid.) Bruch et al. =Loeskeobryum brevirostre

- proliferum (Brid.) Lindb. = Hylocomium splendens

- pyrenaicum (Spruce) Lindb. = Hylocomiastrum pyrenaicum

- splendens var. alaskanum (Lesq. \& James) Limpr. = Hylocomium splendens var. obtusifolium

- umbratum (Hedw.) Bruch et al. = Hylocomiastrum umbratum

Hymenostomum spp. $=$ Weissia spp.

- microstomum (Hedw.) R. Br. = Weissia brachycarpa

- squarrosum Nees \& Hornsch. = Weissia squarrosa

Hypnum arcuatum Lindb. = Calliergonella lindbergii

- bambergeri Schimp. = Stereodon bambergeri

- callichroum Brid. = Stereodon callichrous

- cupressiforme var. ericetorum Bruch et al. = Hypnum jutlandicum

- cupressiforme var. filiforme Brid. = Hypnum cupressiforme

- cupressiforme var. julaceum Brid. = Hypnum cupressiforme

- cupressiforme var. mamillatum Brid. = Hypnum andoi

- cupressiforme var. vaucheri (Lesq.) Boulay = Stereodon vaucheri

- fastigiatum Brid. = Drepanium recurvatum

- fastigiatum var. recurvatum (Lindb. \& Arnell) Uggla = Drepanium recurvatum

- fauriei Cardot $=$ Stereodon fauriei

- fertile Sendtn. = Stereodon fertilis

- hamulosum Bruch et al. = Stereodon hamulosus

- holmenii Ando = Stereodon holmenii

- lacunosum (Brid.) Brid. = Hypnum cupressiforme var. lacunosum 
- leptothallum (Müll.Hal.) Paris = Eurohypnum leptothallum

- lindbergii Mitt. = Calliergonella lindbergii

- mamillatum (Brid.) Loeske = Hypnum ando

_ pallescens (Hedw.) P. Beauv. = Stereodon pallescens

- plicatulum (Lindb.) A.Jaeger = Stereodon plicatulus

- plumaeforme Wilson $=$ Stereodon plumaeformis

— pratense W.D. J.Koch ex Spruce = Breidleria pratensis

- procerrimum Molendo $=$ Stereodon procerrimus

- recurvatum (Lindb. \& Arnell) Kindb. = Drepanium recurvatum

- reptile Michx. $=$ Stereodon pallescens

- revolutum (Mitt.) Lindb. = Stereodon revolutus

- subimponens Lesq. = Stereodon subimponens

- subplicatile Limpr. = Stereodon plicatulus

- tristo-viride (Broth.) Paris = Stereodon tristo-viridis

- vaucheri Lesq. = Stereodon vaucheri

Hypopterygium japonicum Mitt. = Hypopterygium flavolimbatum

Imbribryum alpinum (Huds. ex With.) N.Pedersen = Bryum alpinum

- muehlenbeckii (Bruch et al.) N.Pedersen = Bryum muehlenbeckii

Isopterygium alpicola (Lindb. \& Arnell) Nyholm = Isopterygiopsis alpicola

- alternans Cardot $=$ Taxiphyllum alternans

- elegans (Brid.) Lindb. = Pseudotaxiphyllum elegans

- depressum (Brid.) Mitt. = Taxiphyllum wissgrillii

- muellerianum (Schimp.) A.Jaeger = Isopterygiopsis muelleriana

- nitidum Lindb. = Isopterygiopsis pulchella

- nitidum var. pulchellum (Hedw.) Lindb. = Isopterygiopsis pulchella

- pulchellum (Hedw.) A.Jaeger = Isopterygiopsis pulchella

— pulchellum var. nitidulum (Wahlenb.) G.Roth = Isopterygiopsis pulchella

- turfaceum (Lindb.) Lindb. = Herzogiella turfacea

Isothecium diversiforme (Mitt.) Besch. = Dolichomitriopsis diversiformis

— filescens (Brid.) Mönk. = Plasteurhynchium striatulum

- myurum Brid. = Isothecium alopecuroides

Leptodictyum humile (P. Beauv.) Ochyra= Hygroamblystegium humile

- kochii (Bruch et al.) Warnst. = Hygroamblystegium humile

— trichopodium (Schultz) Warnst. = Hygroamblystegium humile

- trichopodium var. curvipes (Bruch et al.) Broth. = Hygroamblystegium humile

Leptodontium styriacum (Jur.) Limpr. = Leptodontium flexifolium

Lescuraea atrovirens (Dicks. ex Brid.) Kindb. = Lescuraea incurvata

- mutabilis var. saxicola (Bruch et al.) I.Hagen $=$ Lescuraea saxicola

- striata var. saxicola Bruch et al. = Lescuraea saxicola

Leskea grandiretis Lindb. ex Broth.= Lindbergia grandiretis

— incrassata Lindb. ex Broth. = Pseudoleskeella ner- vosa

- rostrata Hedw. = Claopodium rostratum

Leskeella incrassata (Lindb. ex Broth.) Broth. = Pseudoleskeella nervosa

- nervosa (Brid.) Loeske = Pseudoleskeella nervosa

- nervosa var. sibirica Arnell = Pseudoleskeella rupestris

- tectorum (Funck ex Brid.) I.Hagen = Pseudoleskeel la tectorum

Limnobium duriusculum De Not. = Ochyraea duriuscula

Limprichtia cossonii (Schimp.) L.E.Anderson, H.A.Crum \& W.R.Buck $=$ Scorpidium cossonii

- intermedia (Lindb.) Loeske = Scorpidium cossonii

- revolvens (Sw. ex anon.) Loeske $=$ Scorpidium revol vens

— vernicosa (Mitt.) Loeske = Hamatocaulis vernicosus

Lindbergia brachyptera auct. F1. Cauc. non (Mitt.) Kindb. = Lindbergia grandiretis

Loeskeobryum cavifolium (Sande Lac.) M.Fleisch. ex Broth. = Loeskeobryum brevirostre

Lydiaea vlassovii (Laz.) Laz. = Microbryum vlassovii

Meesia trichoides Spruce $=$ Meesia uliginosa

- trifaria H.A.Crum, Steere \& L.E.Anderson = Meesia triquetra

Merceya acutiuscula (Lindb. ex Broth.) Broth. = Scopelophila ligulata

- ligulata (Spruce) Schimp. = Scopelophila ligulata

- ligulata var. acutiuscula (Lindb. ex Broth.) P.C.Chen = Scopelophila ligulata

Metaneckera menziesii (Drumm.) Steere = Neckera menziesii

Microthuidium minutulum (Hedw.) Warnst. = Pelekium minutulum

Mielichhoferia himalayana Mitt. = Bryum caucasicum

- mielichhoferiana var. elongata (Hoppe \& Hornsch. ex Hook.) Wijk \& Margad. = Mielichhoferia elongata

- mielichhoferiana var.japonica (Besch.) Ochi=Mielichhoferia japonica

- nitida Nees \& Hornsch. = Mielichhoferia mielichhoferiana

Mniobryum albicans (Wahlenb.) Limpr. = Pohlia wahlenbergii

- atropurpureum (Wahlenb.) I.Hagen $=$ Pohlia atropur purea

- carneum Limpr. = Pohlia melanodon

- delicatulum (Hedw.) Dixon = Pohlia melanodon

- ludwigii (Spreng. ex Schwägr.) Loeske = Pohlia ludwigii

- pulchellum (Hedw.) Loeske = Pohlia lescuriana

- vexans Limpr. = Pohlia vexans

- wahlenbergii (F.Weber \& D.Mohr) Jenn. = Pohlia wahlenbergii

- wahlenbergii var. glaciale (Brid.) Wijk \& Margad. = Pohlia wahlenbergii

Mnium acutum Lindb. = Plagiomnium acutum

- affine Bland. = Plagiomnium affine

- affine var. elatum Bruch et al. = Plagiomnium elatum

- affine var. integrifolium (Lindb.) Milde = Plagiomnium ellipticum 
- affine var. rugicum (Laurer) Bruch et al. = Plagiomnium ellipticum

— ambiguum $\mathrm{H}$. Müll. = Mnium lycopodioides

- andrewsianum Steere $=$ Rhizomnium andrewsianum

- arcuatum Broth. = Trachycystis ussuriensis

- cinclidioides Huebener $=$ Pseudobryum cinclidioides

- confertidens (Lindb. \& Arnell) Kindb. = Plagiomnium confertidens

- curvatulum $($ Lindb.) Limpr. = Plagiomnium curvatulum

- cuspidatum Hedw. = Plagiomnium cuspidatum

— drummondii Bruch \& Schimp. = Plagiomnium drummondii

- ellipticum Brid. = Plagiomnium ellipticum

- flagellare Sull. \& Lesq. = Trachycystis flagellaris

- hymenophylloides Huebener $=$ Cyrtomnium hymenophylloides

- hymenophyllum Bruch et al. $=$ Cyrtomnium hymenophyllum

- immarginatum Broth. $=$ Trachycystis ussuriensis

- japonicum Lindb. = Plagiomnium japonicum

- lindbergii Smirnova = Mnium lycopodioides

- longirostre Brid. = Plagiomnium rostratum

- magnifolium Horik. = Rhizomnium magnifolium

- magnirete (Lindb. \& Arnell) Kindb. = Mnium lycopodioides

- maximoviczii Lindb. $=$ Plagiomnium maximoviczii

- medium Bruch et al. = Plagiomnium medium

- medium var. curvatulum (Lindb.) G.Roth = Plagiomnium curvatulum

- microovale Müll.Hal. = Plagiomnium maximoviczii

- microphyllum Dozy \& Molk. = Trachycystis microphyllus

- minutulum Besch. $=$ Rhizomnium parvulum

- nudum E.Britton \& R.S.Williams = Rhizomnium nudum

- orthorrhynchum Müll.Hal. = Mnium thomsonii

— pseudopunctatum Bruch \& Schimp. $=$ Rhizomnium pseudopunctatum

- punctatum Hedw. $=$ Rhizomnium punctatum

- punctatum var. elatum Schimp. $=$ Rhizomnium magnifolium

- riparium Mitt. $=$ Mnium lycopodioides

- rostratum Schrad. $=$ Plagiomnium rostratum

- rugicum Laurer $=$ Plagiomnium ellipticum

- seligeri Jur. $=$ Plagiomnium elatum

- serratum Schrad. ex Brid. = Mnium marginatum

- striatulum Mitt. $=$ Rhizomnium striatulum

- submarginatum Nawashin \& N.W.Zinger $=$ Rhizom nium pseudopunctatum

— trichomanes Mitt. = Plagiomnium acutum

- undulatum Hedw. $=$ Plagiomnium undulatum

- vesicatum Besch. $=$ Plagiomnium vesicatum

Myurella apiculata (Sommerf.) Bruch et al. = Myurella tenerrima

Myuroclada rotundifolia (Arnell) Abramova \& I.I.Abramov $=$ Myrinia rotundifolia

Neckera oligocarpa Bruch = Neckera pennata var. tenera

- turgida Jur. $=$ Neckera menziesii

Nyholmiella gymnostoma (Bruch ex Brid.) Holmen \& E. Warncke $=$ Orthotrichum gymnostomum

— obtusifolia (Brid.) Holmen \& E.Warncke = Orthotri- chum obtusifolium

Octodiceras fontanum (Bach. Pyl.) Lindb. $=$ Fissidens fontanus

- julianum (Savi ex DC.) Brid. $=$ Fissidens fontanus

Oncophorus wahlenbergii var. compactus (Bruch et al.) Braithw. = Oncophorus compactus

Oreoweisia bruntonii $(\mathrm{Sm}$.) Milde $=$ Cynodontium bruntonii

Orthodicranum flagellare (Hedw.) Loeske $=$ Dicranum flagellare

- fragilifolium $($ Lindb.) Podp. $=$ Dicranum fragilifolium

- hamulosum (Mitt.) Broth. = Dicranum hamulosum

- mayrii (Broth.) Smirnova = Dicranum mayrii

- montanum (Hedw.) Loeske $=$ Dicranum montanum

- strictum auct. $=$ Dicranum tauricum

- tauricum (Sapjegin) Smirnova = Dicranum tauricum

Orthotheciella varia (Hedw.) Ochyra $=$ Hygroamblystegium varium

Orthodontium australe Hook.f. \& Wilson = Orthodontium lineare

Orthotrichum australe Jur. = Orthotrichum tenellum

- caucasicum Venturi $=$ Orthotrichum sordidum

- cupulatum var. nudum (Dicks.) Lindb. = Orthotrichum cupulatum var. riparium

— elegans Schwägr. ex Hook. \& Grev. = Orthotrichum speciosum

- fallax Bruch ex Brid. = Orthotrichum pumilum

- fastigiatum Bruch ex Brid. = Orthotrichum affine

- killiasii Müll.Hal. = Orthotrichum speciosum

- laevigatum var. japonicum (Z.Iwats.) Lewinsky = Orthotrichum iwatsukii

- limprichtii I.Hagen = Orthotrichum cupulatum

- nudum Dicks. = Orthotrichum cupulatum var. riparium

- pallidum Gronvall, nom. illeg. = Orthotrichum pallens

- philibertii Venturi = Orthotrichum pumilum

- saxatile Schimp. $=$ Orthotrichum anomalum

- sibiricum Gronvall = Orthotrichum pallens

- schimperi Hammar = Orthotrichum pumilum

Oxyrrhynchium praelongum (Hedw.) Warnst. $=$ Kindber gia praelonga

Oxystegus cylindricus (Bruch ex Brid.) Hilp. $=$ Oxystegus tenuirostris

Pachyfissidens grandifrons $($ Brid.) Limpr. $=$ Fissidens grandifrons

Palustriella commutata var. falcata (Brid.) Ochyra $=$ Palustriella falcata

- commutata var. fallax (Brid.) Ochyra = Palustriella commutata

Paraleucobryum longifolium var. sauteri (Bruch et al.) C.E.O.Jensen $=$ Paraleucobryum sauteri

Phascum acaulon With. = Tortula acaulon

- curvicolle Hedw. = Microbryum curvicollum

- cuspidatum Hedw. = Tortula acaulon

- cuspidatum var. piliferum (Hedw.) Hook. \& Taylor $=$ Tortula acaulon

- floerkeanum F.Weber \& D.Mohr $=$ Microbryum floerkeanum

- piliferum Hedw. $=$ Tortula acaulon

- vlassovii Laz. $=$ Microbryum vlassovii

Philonotis capillaris auct. non Lindb. $=$ Philonotis arnellii 
- fontana var. caespitosa (Jur.) Limpr. = Philonotis caespitosa

- fontana var. falcata auct. non (Hook.) Brid. = Philonotis calcarea

- fontana var. pumila (Turner) Brid. = Philonotis tomentella

- fontana var. seriata (Mitt.) Kindb. = Philonotis seriata

Physcomitrium acuminatum Bruch et al. = Physcomitrium eurystomum subsp. acuminatum

- martjanovii Broth. ex I.I.Abramov = Entosthodon hungaricus

- patens (Hedw.) Mitt. = Physcomitrella patens

Plagiobryum bimum (Schreb.) N.Pedersen = Bryum bimum

- capillare $($ Hedw.) N.Pedersen = Bryum capillare

- cyclophyllum (Schwägr.) N.Pedersen = Bryum cyclophyllum

- demissum subsp. hultenii Ochi \& Perss. = Plagiobryum hultenii

- pallens (Sw. ex anon.) N.Pedersen $=$ Bryum pallens

- pallescens (Schleich. ex Schwägr.) N.Pedersen = Bryum pallescens

- pseudotriquetrum (Hedw.) N.Pedersen (incl. B. pseudocrispulum) $=$ Bryum pseudotriquetrum

- purpurascens $(\mathrm{R} . \mathrm{Br}$.) N.Pedersen = Bryum purpurascens

- uliginosum (Brid.) N.Pedersen = Bryum uliginosum

- wrightii (Sull. \& Lesq.) N.Pedersen = Bryum wrightii

Plagiomnium medium subsp. curvatulum (Lindb.) T.J.Kop. $=$ Plagiomnium curvatulum

Plagiopus oederi (Brid.) Limpr. = Plagiopus oederianus Plagiotheciella latebricola (Bruch et al.) M.Fleisch. = Plagiothecium latebricola

— pilifera (Sw. ex Hartm.) M.Fleisch. = Plagiothecium piliferum

Plagiothecium denticulatum var. curvifolium (Schlieph. ex Limpr.) Meylan = Plagiothecium curvifolium

- denticulatum var. laetum (Bruch et al.) Lindb. = Plagiothecium laetum

- denticulatum var. ruthei (Limpr.) Riehm. = Plagioth ecium denticulatum var. undulatum

- depressum (Brid.) Spruce = Taxiphyllum wissgrillii

- donnianum (Sm.) Mitt. = Plagiothecium denticulatum

- laetum var. curvifolium (Limpr.) Mastracci \& M.Sauer = Plagiothecium curvifolium

- neglectum Mönk. = Plagiothecium nemorale

- roeseanum Bruch et al. = Plagiothecium cavifolium

- ruthei Limpr. = Plagiothecium denticulatum var. undulatum

- silesiacum (F.Weber \& D.Mohr) Bruch et al. = Herzogiella seligeri

- sylvaticum (Brid.) Bruch et al. = Plagothecium spp.

Platydictya baicalensis (Ignatov \& Ochyra) Hedenäs = Bardunowia baicalensis

- confervoides (Brid.) H.A.Crum = Serpoleskea confervoides

- subtilis (Hedw.) H.A.Crum = Serpoleskea subtilis

Platyhypnidium riparioides (Hedw.) Dixon $=$ Rhynchostegium riparioides
Pleuridium alternifolium auct. non (Dicks. ex Hedw.) Rabenh. = Pleuridium subulatum

- palustre (Bruch \& Schimp.) Bruch et al. = Cleistocarpidium palustre

Pleuropus euchloron (Müll.Hal.) Broth. = Palamocladium euchloron

Pleuroweisia schliephackei Limpr. ex Schlieph. = Molendoa schliephackei

Pogonatum alpinum (Hedw.) Röhl. = Polytrichastrum alpinum

- capillare $($ Michx.) Brid. = Pogonatum dentatum

- grandifolium $($ Lindb.) A.Jaeger = Pogonatum japonicum

- laterale Brid. = Pogonatum contortum

Pohlia acuminata auct. = Pohlia elongata var. greenii

- ambigua (Limpr.) Broth. = Pohlia elongata var. greenii

- betulina Warnst. = Pohlia nutans

- carnea (Schimp.) Lindb. = Pohlia melanodon

- delicatula (Hedw.) Grout = Pohlia melanodon

- drummondii var. gracilis (Bruch et al.) Podp. = Pohlia filum

- elongata var. acuminata auct. = Pohlia elongata var. greenii

- elongata var. acuminata (Hoppe \& Hornsch.) Huebener $=$ Pohlia elongata

- elongata var. minor Hartm. = Pohlia elongata var. greenii

- elongata var. polymorpha (Hoppe \& Hornsch.) Nyholm $=$ Pohlia elongata var. greenii

- filiformis (Dicks.) A.L.Andrews = Anomobryum julaceum

- gracilis (Bruch et al.) Lindb. = Pohlia filum

- gracillima (Cardot) Horik. \& Ochi = Pohlia leucostoma

- minor auct. = Pohlia elongata var. greenii

- polymorpha auct. non Hoppe \& Hornsch. = Pohlia elongata var. greenii

- polymorpha Hoppe \& Hornsch. = Pohlia elongata

- pulchella (Hedw.) Lindb. = Pohlia lescuriana

- rothii auct. non (Correns ex Limpr.) Broth. = Pohlia andalusica

- schimperi (Müll.Hal.) A.L.Andrews = Pohlia nutans var. schimperi

- wahlenbergii var. glacialis (Brid.) E.F.Warb. = Pohlia wahlenbergii

Polytrichastrum longisetum var. anomalum (Milde) Ignatov \& G.L.Sm. = Polytrichastrum longisetum

- norwegicum (Hedw.) Schljakov = Polytrichastrum alpinum var. septentrionale

Polytrichum affine Funck = Polytrichum strictum

- alpestre Hoppe $=$ Polytrichum strictum

- alpinum Hedw. = Polytrichastrum alpinum

- commune var. humile Sw. = Polytrichum commune

- - var. nigrescens Warnst. = Polytrichum swartzii

- - var. perigoniale (Michx.) Hampe = Polytrichum commune

— - var. swartzii (Hartm.) Nyholm $=$ Polytrichum swartzii

— - var. uliginosum Wallr. = Polytrichum commune $\{151\}$ 
- decipiens Limpr. = Polytrichastrum pallidisetum

- formosum Hedw. = Polytrichastrum formosum

- formosum var. decipiens (Limpr.) Loeske = Polytrichastrum pallidisetum

- fragile Bryhn = Polytrichastrum alpinum var. fragile

- gracile Dicks. $=$ Polytrichastrum longisetum

- juniperinum var. affine (Funck) Brid. = Polytrichum strictum

- juniperinum var. alpestre (Hoppe) Röhl. = Polytrichum strictum

- longisetum Sw. ex Brid. = Polytrichastrum longisetum

- norvegicum auct. = Polytrichastrum sexangulare

- ohioense auct. non Renauld \& Cardot = Polytrichas trum pallidisetum

- pallidisetum Funck $=$ Polytrichastrum pallidisetum

- perigoniale Michx. = Polytrichum commune

- sexangulare Floerke ex Brid. = Polytrichastrum sexangulare

— sphaerothecium (Besch.) Müll.Hal. = Polytrichastrm sphaerothecium

- strictum var. alpestre (Hoppe) Rabenh. = Polytrichum strictum

Pottia angustifolia $($ Lindb.) Paris = Microbryum davallianum

- bryoides (Dicks.) Mitt. = Tortula protobryoides

- caucasica (Lindb. ex Broth.) Paris = Tortula caucasica

- curvicollis (Hedw.) Mitt. = Microbryum curvicolle

- davalliana (Sm.) C.E.O.Jensen = Microbryum daval lianum

- davalliana var. angustifolia (Lindb. ex Broth.) Podp. = Microbryum davallianum

- heimii (Hedw.) Hampe = Hennediella heimii

- heimii var. obtusifolia (Müll.Hal.) I.Hagen = Hennediella heimii var. arctica

- intermedia (Turner) Fürnr. = Tortula modica

- lanceolata (Hedw.) Müll.Hal. = Tortula lanceola

- lindbergii (Kindb.) Warnst. = Tortula lindbergii

- mutica Venturi = Microbryum davallianum

- recta (With.) Mitt. = Microbryum rectum

— rufescens Müll.Hal. = Microbryum davallianum

- starckeana (Hedw.) Müll.Hal.= Microbryum starckeanum

- truncata (Hedw.) Bruch et al. = Tortula truncata

Protobryum bryoides (Dicks.) J.Guerra \& M.J.Cano = Tortula protobryoides

Pseudisothecium myosuroides (Brid.) Grout = Isothecium myosuroides

Pseudoleskea atrovirens (Dicks. ex Brid.) Bruch et al. = Lescuraea incurvata

- chilensis (Lorentz) Ochyra = Hygroamblystegium varium

- decipiens (Limpr.) Kindb. = Lescuraea saxicola

- filamentosa (Dicks. ex With.) C.E.O.Jensen = Lescuraea incurvata

- incurvata (Hedw.) Loeske = Lescuraea incurvata

- korjakorum Laz. = Myrinia pulvinata

- patens (Lindb.) Kindb. = Lescuraea patens

- radicosa (Mitt.) Macoun \& Kindb. = Lescuraea radi$\cos a$

- saviana (De Not.) Latzel = Lescuraea saviana

- secunda (Arnell) Broth. = Lescuraea secunda

- tectorum (Funck ex Brid.) Schimp.= Pseudoleskeella tectorum

Pseudoleskeella nervosa var. rupestris (Berggr.) Nyholm

= Pseudoleskeella rupestris

- nervosa var. sibirica (Arnell) E.Lawton = Pseudoleskeella rupestris

— sibirica (Arnell) P.S.Wilson \& D.H.Norris = Pseudoleskeella rupestris

Pseudostereodon procerrimus (Molendo) M.Fleisch. = Stereodon procerrimus

Psilopilum falcatum (Steere) H.A.Crum, Steere \& L.E.Anderson = Oligotrichum falcatum

Pterigynandrum filiforme var. decipiens (F.Weber \& D.Mohr) Limpr. = Pterigynandrum filiforme

Pterygoneurum arcticum Steere = Pterygoneurum lamel latum

- cavifolium Jur. = Pterygoneurum ovatum

- medium (E.S.Salmon) Broth. = Pterygoneurum ovatum

Ptychodium plicatum (Schleich. ex F.Weber \& D.Mohr) Schimp. = Lescuraea plicata

Pylaisia intricata auct. non (Hedw.) Bruch et al. = Pylaisia stereodontoides

- schimperi Cardot $=$ Pylaisia selwynii

Pylaisiella spp. $=$ Pylaisia spp.

Racomitrium aciculare (Hedw.) Brid. = Codriophorus acicularis

- affine (Schleich. ex F.Weber \& D.Mohr) Lindb. = Bucklandiella affinis

- afoninae Frisvoll = Bucklandiella afoninae

- aquaticum (Brid. ex Schrad.) Brid. = Codriophorus aquaticus

- barbuloides Cardot $=$ Niphotrichum barbuloides

- brevisetum Lindb. = Codriophorus brevisetus

- canescens (Hedw.) Brid. = Niphotrichum canescens

- canescens var. epilosum H.Müll. ex Milde = Niphotrichum ericoides

- canescens var. ericoides (Brid.) Hampe = Niphotrichum ericoides

- canescens var. latifolium Lange \& C.E.O.Jensen = Niphotrichum canescens

- canescens var. robustum (Lindb. \& Arnell) Paris = Niphotrichum panschii

- canescens var. strictum Schlieph. = ? Niphotrichum ericoides

- canescens var. vulgare Loeske = Niphotrichum canescens

- elongatum Ehrh. ex Frisvoll = Niphotrichum elongatum

- ericoides (Brid.) Brid. = Niphotrichum ericoides

- fasciculare (Hedw.) Brid. = Codriophorus fascicularis

- fontinaloides (Hedw.) Brid. = Cinclidotus fontinaloides

- heterostichum (Hedw.) Brid. = Bucklandiella heterosticha

- heterostichum var. affine (Schleich. ex F.Weber \& D.Mohr) Lesq. = Bucklandiella affinis

- heterostichum var. alopecurum Huebener = Bucklandiella affinis

- heterostichum var. gracilescens Bruch et al. = Bucklandiella heterosticha

- heterostichum var. microcarpon (Hedw.) Boulay = Bucklandiella microcarpa 
- heterostichum var. sudeticum (Funck) E.Bauer $=$ Bucklandiella sudetica

- hypnoides Lindb. = Racomitrium lanuginosum

- japonicum Dozy \& Molk. = Niphotrichum japonicum

- laetum Besch. \& Cardot = Bucklandiella laeta

- macounii subsp. alpinum (E.Lawton) Frisvoll = Bucklandiella macounii subsp. alpina

- microcarpon (Hedw.) Brid. = Bucklandiella microcarpa

- muticum (Kindb.) Frisvoll = Niphotrichum muticum

- panschii (Müll.Hal.) Kindb. = Niphotrichum panschii

- patens (Hedw.) Huebener = Grimmia ramondii

- ramulosum Lindb. = Bucklandiella microcarpa

- riparium (Host ex Brid.) Brid. = Cinclidotus riparius

- sudeticum (Funck) Bruch et al. = Bucklandiella sudetica

- virescens Lindb. = Codriophorus fascicularis

Rhabdoweisia kusenevae Broth. = Rhabdoweisia crispata

Rhizomnium perssonii T.J.Kop. = Rhizomnium magnifolium

- punctatum var. elatum (Schimp.) T.J.Kop. = Rhizomnium magnifolium

Rhodobryum spathulatum (Hornsch.) Pocz $=$ Rhodobryum ontariense

Rhynchostegiella compacta (Drumm. ex Müll.Hal.) Loeske) = Conardia compacta

- jacquinii (Garov.) Limpr. = Rhynchostegiella teneriffae

- pallidirostris (Brid.) Loeske $=$ Oxyrrhynchium pumilum

- pumila (Wilson) E.F.Warb. = Oxyrrhynchium pumilum

- teesdalei (Bruch et al.) Limpr. = Rhynchostegiella teneriffae

Rhynchostegium murale var. arcticum I.Hagen = Rhynchostegium arcticum

Rhytidiadelphus squarrosus var. calvescens (Kindb.) Warnst. = Rhytidiadelphus subpinnatus

Rhytidiastrum squarrosum (Hedw.) Ignatov \& Ignatova = Rhytidiadelphus squarrosus

- subpinnatum (Lindb.) Ignatov \& Ignatova = Rhytidiadelphus subpinnatus

Sanionia nivalis Hedenäs = Sanionia georgicouncinata

Sarmentypnum exannulatum (Bruch et al.) Hedenäs = Warnstorfia exannulata

- procerum (Renauld \& Arnell) Hedenäs = Warnstorfia procera

- pseudosarmentosum (Cardot \& Thér.) Hedenäs = Warnstorfia pseudosarmentosa

- sarmentosum (Wahlenb.) Tuom. \& T.J.Kop. = Warnstorfia sarmentosa

- trichophyllum (Warnst.) Hedenäs = Warnstorfia trichophylla

- tundrae (Arnell) Hedenäs = Warnstorfia tundrae

Saviczia obtusissima (Broth.) Abramova \& I.I.Abramov = Plagiothecium obtusissimum

Schistidium alpicola auct. non (Hedw.) Limpr. = Schistidium platyphyllum

- alpicola var. latifolia (J.E.Zetterst.) Limpr. = Schistidium platyphyllum

- alpicola var. rivulare (Brid.) Limpr. = Schistidium rivulare

- anodon (Bruch et al.) Loeske $=$ Grimmia anodon
- apocarpum f. submuticum Broth., nom. nud. = Schistidium submuticum

- apocarpum subsp. papillosum (Culm.) Poelt $=$ Schistidium papillosum

— apocarpum subsp. singarense (Schiffn.) Podp. $=$ Schistidium helveticum

- apocarpum var. abrupticostatum Bryhn = Schistidium platyphyllum subsp. abrupticostatum

- apocarpum var. boreale (Poelt) Düll = Schistidium boreale

- apocarpum var. brunnescens (Limpr.) Loeske $=$ Schistidium brunnescens

- apocarpum var. confertum (Funck) H.Möller $=$ Schistidium confertum

- apocarpum var. didymontoides Loeske \& L.I.Savicz = Schistidium platyphyllum subsp. abrupticostatum

- apocarpum var. gracile (Röhl.) Bruch et al. = Schistidium spp.

- gracile (Röhl.) Limpr. $=$ Schistidium spp.

- pulvinatum var.flaccidum (De Not.) De Not. = Schistidium flaccidum

- rivulare var. latifolium (J.E.Zetterst.) H.A.Crum \& L.E.Anderson $=$ Schistidium platyphyllum

- rivulare subsp. latifolium (J.E.Zetterst.) B.Bremer $=$ Schistidium platyphyllum

- singarense (Schiffn.) Laz. = Schistidium helveticum

- strictum auct. non (Turner) Loeske ex Mårtensson = Schistidium spp.

Scleropodiopsis laxiretis Ignatov $=$ Rhynchostegium arcticum Scleropodium apiculigerum (Lindb. \& Arnell) J.-P.Frahm = Sciuro-hypnum ornellanum

- ornellanum (Molendo) Lorentz = Sciuro-hypnum ornellanum

- purum (Hedw.) Limpr. $=$ Pseudoscleropodium purum

Scorpidium turgescens (T.Jensen) Loeske $=$ Pseudocalliergon turgescens

Scouleria pulcherrima Broth. = Scouleria aquatica var. pulcherrima

- rschewinii Lindb. \& Arnell = Scouleria aquatica

Seligeria alpestris T.Schauer =Seligeria patula

- patula var. alpestris (T.Schauer) Gos \& Ochyra = Seligeria patula

- pusilla var. brevifolia Lindb. = S. brevifolia

— tristicha (Brid.) Bruch et al. = Seligeria trifaria

- tristichoides var. patula (Lindb.) Broth.= Seligeria patula

Semibarbula trachyphylla (Broth.) Laz. ex Mamatkulov = Tortula trachyphylla

Sharpiella seligeri (Brid.) Z.Iwats. = Herzogiella seligeri

— turfacea (Lindb.) Z.Iwats. = Herzogiella turfacea

Sphagnum acutifolium Schrad. = Sphagnum capillifolium

- affine var. flagellare (Schlieph. ex Röll) L.Söderstr. \& Hedenäs = Sphagnum affine

- amblyphyllum (Russow) Warnst. = Sphagnum flexuosum

- apiculatum H.Lindb. = Sphagnum fallax

- auriculatum var. inundatum (Russow) M.O.Hill = Sphagnum inundatum

- capillaceum (Weiss) Schrank = Sphagnum capillifolium

- capillifolium var. tenellum (Schimp.) H.A.Crum = Sphagnum rubellum 
- cuspidatum var. majus Russow = Sphagnum majus

- cymbifolium (Ehrh.) Hedw. = Sphagnum palustre

- denticulatum Brid. = Sphagnum auriculatum

- denticulatum var. inundatum (Russow) Kartt. = Sphagnum inundatum

- dusenii (C.E.O.Jensen) Russow \& Warnst. = Sphagnum majus

— imbricatum var. arcticum Flatberg = Sphagnum steerei

- imbricatum subsp. affine (Renauld \& Cardot) Flatberg = Sphagnum affine

— imbricatum subsp. austinii (Sull.) Flatberg = Sphagnum austinii

- lescurii auct. non Sull. = Sphagnum auriculatum

- lescurii var. inundatum (Russow) Düll = Sphagnum inundatum

- medium Limpr. = Sphagnum magellanicum

- molluscum Bruch = Sphagnum tenellum

- mucronatum (Russow) Zick. = Sphagnum fallax

- nemoreum Scop. = Sphagnum capillifolium

- obtusum var. dusenii C.E.O.Jensen $=S$. majus

- parvifolium (Sendtn. ex Warnst.) Warnst. = Sphagnum angustifolium

— plumulosum Röll. = Sphagnum subnitens

- recurvum subsp. balticum Russow $=$ Sphagnum balticum

- recurvum var. amblyphyllum (Russow) Warnst. = Sphagnum flexuosum

- recurvum var. angustifolium C.E.O.Jensen ex Russow = Sphagnum angustifolium

- recurvum var. mucronatum (Russow) Warnst. = Sphagnum fallax

- recurvum var. parvifolium Sendtn. ex Warnst. $=$ Sphagnum angustifolium

- recurvum var. tenue H.Klinggr. = Sphagnum angustifolium

— rigidum (Nees \& Hornsch.) Schimp. = Sphagnum compactum

- robustum (Warnst.) Röll = Sphagnum russowii

- rufescens (Nees \& Hornsch.) Warnst. = Sphagnum auriculatum

- subbicolor auct. non Hampe = Sphagnum centrale

- subsecundum var. auriculatum (Schimp.) Schlieph. = Sphagnum auriculatum

- subsecundum var. contortum (Schultz) Huebener = Sphagnum contortum

- subsecundum subsp. inundatum (Russow) Meyl. = Sphagnum inundatum

- subsecundum var. inundatum (Russow) C.E.O.Jensen = Sphagnum inundatum

- subsecundum var. rufescens (Nees \& Hornsch.) Huebener = Sphagnum auriculatum

- subtile (Russow) Warnst. = Sphagnum capillifolium

- warnstorfianum Du Rietz = Sphagnum warnstorfii

- zickendrathii Warnst. = Sphagnum obtusum

Splachnum luteum var. melanocaulon Wahlenb. = Splachnum melanocaulon

- mnioides Hedw. = Tetraplodon mnioides

- ovatum Hedw. = Splachnum sphaericum

Sporledera palustris (Bruch \& Schimp.) Hampe = Cleistocarpidium palustre
Stegonia latifolia var. pilifera (Brid.) Broth. = Stegonia pilifera

Stellariomnium spp. $=$ Mnium spp.

Stereodon arcuatus Lindb. = Calliergonella lindbergii

- cupressiformis (Hedw.) Brid. ex Mitt. = Hypnum cupressiforme

- cupressiformis var. vaucheri (Lesq.) Lindb. \& Arnell = Stereodon vaucheri

- fastigiatus (Brid.) Braithw. = Drepanium recurvatum

- recurvatus Lindb. \& Arnell = Drepanium recurvatum

Streblotrichum convolutum (Hedw.) P. Beauv. = Barbula convoluta

- croceum (Brid.) Loeske $=$ Barbula crocea

- enderesii (Garov.) Loeske = Barbula enderesii

- flavipes (Bruch et al.) J.J.Amann = Barbula enderesii

Streptocolea atrata (Miel. ex Hornsch.) Ochyra \& Zarnowiec $=$ Grimmia atrata

Stroemia gymnostoma (Brid.) I.Hagen = Orthotrichum gymnostomum

- obtusifolia (Brid.) I.Hagen = Orthotrichum obtusifolium

Struckia argentata subsp. zerovii (Laz.) Tan et al. = Struckia enervis

— zerovii (Laz.) Hedenäs = Struckia enervis

Syntrichia alpina (Bruch et al.) Jur. = Syntrichia sinensis

- Syntrichia densa (Velen.) J.-P.Frahm = Syntrichia calcicola

- desertorum (Broth.) J.J.Amann = Syntrichia caninervis

- ferganensis (Laz.) Laz. = Syntrichia handelii var. ferganensis

- inermis (Brid.) Bruch $=$ Tortula inermis

- intermedia Brid. = Syntrichia montana

- montana subsp. handelii (Schiffn.) Podp. = Syntrichia handelii

- mucronifolia (Schwägr.) Brid. = Tortula mucronifolia

— pagorum (Milde) J.J.Amann = Syntrichia laevipila

- pulvinata (Jur.) Jur. = Syntrichia virescens

- ruraliformis (Besch.) Cardot $=$ Syntrichia ruralis var. ruraliformis

- ruralis var. arenicola J.J.Amann, nom. illeg. = Syntrichia ruralis var. ruraliformis

- ruralis var. hirsuta (Venturi) Podp. = Syntrichia papillosissima

- subulata (Hedw.) F.Weber \& D.Mohr = Tortula subulata

Tayloria serrata var. tenuis (Dicks.) Bruch et al. = Tayloria tenuis

- splachnoides var. acuminata (Hornsch.) Huebener $=$ Tayloria acuminata

Tetraplodon bryoides Lindb. = Tetraplodon mnioides

Tetrodontium brownianum var. ovatum (Funck) Wijk \& Marg. = Tetrodontium ovatum

- brownianum var. repandum (Funck) Limpr. = Tetrodontium repandum

Thamnium spp. = Thamnobryum spp.

Thamnobryum sandei (Besch.) Z.Iwats. = Thamnobryum subseriatum

Thuidium abietinum (Hedw.) Bruch et al. = Abietinella abietina 
- abietinum var. hystricosum (Mitt.) Loeske \& Lande = Abietinella abietina

- bipinnatulum Mitt. = Pelekium versicolor

- delicatulum var. radicans (Kindb.) H.A.Crum, Steere \& L.E.Anderson $=$ Thuidium assimile

- erectum Duby $=$ Thuidium delicatulum

- glaucinum (Mitt.) Bosch \& Sande Lac. = Thuidium pristocalyx

- gracile Bruch et al. = Haplocladium microphyllum

- hystricosum Mitt. = Abietinella abietina

- lanatum (P.Størm ex Brid.) I.Hagen = Helodium blandowii

- minutulum (Hedw.) Bruch et al. = Pelekium minutulum

- molkenboeri Sande Lac.=Bryonoguchia molkenboeri

- philibertii Limpr. $=$ Thuidium assimile

- pygmaeum Bruch et al. = Pelekium pygmaeum

- vestitissimum Besch. = Bryochenea vestitissima

Timmia austriaca var. arctica (Kindb.) Arnell = Timmia austriaca

- cucullata Michx. = Timmia megapolitana

- elegans I.Hagen = Timmia comata

- megapolitana subsp. bavarica (Hessl.) Brassard = Timmia bavarica

- megapolitana var. bavarica (Hessl.) Brid. = Timmia bavarica

- norvegica var. excurrens Bryhn = Timmia comata

Timmiella corniculata (Wahlenb.) Broth. = ?Timmiella barbuloides $-\{195\}$

Tortula aestiva (Hedw.) P. Beauv. = Tortula muralis var. aestiva

- angustifolia Lindb. ex Broth. = Microbryum davallianum

- calcicolens W.A.Kramer = Syntrichia calcicola

- caninervis (Mitt.) Broth. = Syntrichia caninervis

- densa (Velen.) J.-P.Frahm = Syntrichia calcicola

- desertorum Broth. = Syntrichia caninervis

- euryphylla R.H.Zander = Tortula hoppeana

- handelii Schiffn. = Syntrichia handelii

- handelii var. ferganensis (Laz.) W.A.Kramer $=$ Syntrichia handelii var. ferganensis

- heimii (Hedw.) Mitt. = Hennediella heimii

- heimii var. longiseta Lindb. \& Arnell = Hennediella heimii

- hirsuta (Venturi) Laz. = Syntrichia papillosissima

- intermedia (Brid.) De Not. = Syntrichia montana

- intermedia subsp. handelii (Schiffn.) Wijk \& Margad. = Syntrichia handelii

— laevipila (Brid.) Schwägr. = Syntrichia laevipila

- laevipila var. propagulifera Lindb. = Syntrichia laevipila

- lamellata Lindb. = Pterygoneurum lamellatum

- latifolia Bruch ex Hartm. = Syntrichia latifolia

- montana Mitt. = Syntrichia montana

- norvegica (F.Weber) Lindb. = Syntrichia norvegica

- pagorum (Milde) De Not. = Syntrichia laevipila

- papillosa Wilson = Syntrichia papillosa

- papillosissima (Copp.) Broth. = Syntrichia papillosissima

— princeps De Not. = Syntrichia princeps

— pseudohandelii J.Fröhl. = Syntrichia pseudohandelii
- pulvinata (Jur.) Limpr. = Syntrichia virescens

- revolvens (Schimp.) G.Roth $=$ Tortula fiorii

- ruraliformis (Besch.) Ingham = Syntrichia ruralis var. ruraliformis

- ruraliformis var. subpapillosissima (Bizot \& R.B.Pierrot) W.A.Kramer = Syntrichia ruralis var. subpapillosissima

- ruralis (Hedw.) P.Gaertn., B.Mey. \& Scherb. = Syntrichia ruralis

- ruralis subsp. hirsuta (Venturi) W.A.Kramer $=$ Syntrichia papillosissima

- ruralis var. alpina Wahlenb. = Syntrichia norvegica

- ruralis var. arenicola Braithw. = Syntrichia ruralis var. ruraliformis

- ruralis var. calcicola (J.J.Amann) Barkman = Syntrichia calcicola

- ruralis var. crinita De Not. = Syntrichia montana

- ruralis var. densa Velen. = Syntrichia calcicola

- ruralis var. hirsuta (Venturi) Paris = Syntrichia papillosissima

- ruralis var. ruraliformis (Besch.) De Wild. = Syntrichia ruralis var. ruraliformis

- ruralis var. virescens De Not. = Syntrichia virescens

- sinensis (Müll.Hal.) Broth. = Syntrichia sinensis

- subulata var. laevifolia Lindb. = Tortula mucronifolia

- velenovskyi Schiffn. = Hilpertia velenovskyi

- virescens (De Not.) De Not. = Syntrichia virescens

Trichodon cylindricus (Hedw.) Schimp. = Ditrichum cylindricum

Trichostomopsis aaronis (Lorentz) S.Agnew \& C.C.Towns. = Didymodon australasiae

- australasiae (Hook. \& Grev.) H.Rob. = Didymodon australasiae

Trichostomum brevifolium Sendtn. ex Müll.Hal. = Trichostomum crispulum

- crispulum var. viridulum (Bruch) Dixon = Trichostomum viridulum

— cylindricum (Bruch ex Brid.) Müll.Hal. = Oxystegus tenuirostris

- tenuirostre (Hook. \& Taylor) Lindb. = Oxystegus tenuirostris

Ulota americana (P.Beauv.) Limpr. = Ulota hutchinsiae

- crispula Bruch = Ulota crispa

Usmania campylopoda Laz. = Campylostelium pitardii

Warnstorfia fluitans var. falcata (Sanio ex C.E.O.Jensen) H.A.Crum \& L.E.Anderson = Warnstorfia fliutans

- h-schulzei (Limpr.) Loeske = Warnstorfia fluitans

- kurilensis (Smirnova) Schljakov = Warnstorfia fluitans

Weissia controversa var. crispata (Nees \& Hornsch) Nyholm $=$ Weissia fallax

- controversa var. wimmeriana (Sendtn.) Blockeel \& A.J.E.Sm. = Weissia wimmeriana

- microstoma (Hedw.) Müll.Hal. = Weissia brachycarpa

- platyphylla Broth. = Weissia planifolia

- triumphans (De Not.) M.O.Hill = Trichostomum triumphans

- tortilis (Schwägr.) Müll.Hal. = Weissia condensa

- Zygodon baumgartneri Malta = Zygodon rupestris

- viridissimus var. dentatus Limpr. = Zygodon dentatus

— viridissimus var. rupestris Hartm. = Zygodon rupestris 


\section{LITERATURE CITED}

1. [ABOLINA, А.] АБОЛИНЬ А.А. 1965. Листостебельные мхи Латвийской CCP. - [Mosses of Latvian SSR] Riga, $331 \mathrm{pp}$.

2. ABOLINA, A. 1975. Vismazaka süna Latvija. - [The smallest moss in Latvia] Padomju Jaunatne 39.

3. ABOLINA, A. 2001. Latvijas sünu saraksts. - [List of bryophytes of Latvia] Latvijas Vegetâcija 3: 47-87.

4. ABOLINA, A., unpublished data.

5. ABOLINA, A. \& I. RERIHA 2005. West-Latvian bryophytes - the peculiarities of separate species distribution and novelties. - В кн.: «Актуальные проблемы бриологии», Тр. междунар. сов. посв. 90-летию со дня рожд. А.Л. Абрамовой. СПб, 22-25 ноября 2005. [In: Proc. Int. Conf. "Actual Problems of Bryology" devoted to $90^{\text {th }}$ Anniversary of A.L. Abramova. St. Petersburg, 22-25 November 2005]: 9-13.

6. [ABRAMOV, I.I. \& K.Yu. AВACHЕV] АБРАМОВ И.И., К.Ю. АБАЧЕВ 1968. О мхах Дагестана. - [On the bryophytes of Dagestan] Новости сист. низи. pacm. [Novosti Sist. Nizsh. Rast.] '1968': 311-322.

7. [ABRAMOV, I.I., A.L. ABRAMOVA \& I.V. CZERNYADJЕVA] АБРАМОВ И.И., А.Л. АБРАМОВА, И.В. ЧЕРНЯДЬЕВА 1990. Tortula velenovskyi Schiffn. новый вид с плато Путорана (Среднесибирское плоскогорье). - [Tortula velenovskyi Schiffn. - a new species from the Putorana Plateau (Central-Siberian Highland)] Новости сист. низи. pacm. [Novosti Sist. Nizsh. Rast.] 27: 118-124.

8. [ABRAMOV, I.I., A.L. ABRAMOVA \& I.V. SIROTINA] АБРАМОВ И.И., А.Л. АБРАМОВА, И.В. СИРОТИНА 1986. Новые и интересные виды мхов из Туркмении. [New and interesting mosses from the Turkmenistan] Новости сист. низш. pacm. [Novosti Sist. Nizsh. Rast.] 23: $197-204$.

9. [ABRAMOV, I.I., A.L. ABRAMOVA \& I.V. SIROTINA] АБРАМОВ И.И., А.Л. АБРАМОВА, И.В. СИРОТИНА 1987. Крымско-кавказские связи флоры мхов Туркмении. - [The Crimea-Caucasian relationships of the moss flora of the Turkmenistan] Новости сист. низи. раст. [Novosti Sist. Nizsh. Rast.] 24: 179-184.

10. [ABRAMOV, I.I., A.L. ABRAMOVA \& I.V. SIROTINA] АБРАМОВ И.И., А.Л. АБРАМОВА, И.В. СИРОТИНА 1989. О видах рода Entosthodon Schwaegr. (сем. Funariaceae) из Средней Азии. - [On the species of the genus Entosthodon Schwaegr. (family Funariaceae) from the Middle Asia] Hовости сист. низи. pacm. [Novosti Sist. Nizsh. Rast.] 26: 124-132.

11. [ABRAMOV, I.I., A.L. ABRAMOVA \& I.V. SIROTINA] АБРАМОВ И.И., А.Л. АБРАМОВА, И.В. СИРОТИНА 1989. К бриофлоре Туркмении. 2. - [On the bryoflora of the Turkmenistan. 2] Новости сист. низи. pacm. [Novosti Sist. Nizsh. Rast.] 26: 133-136.

11a. [ABRAMOV, I. I. \& L. A. VOLKOVA] АБРАМОВ, И.И., Л.А. ВОЛКОВА 1998. Определитель листостебельных мхов Карелии. - [Handbook of mosses of Karelia] Arctoa 7, suppl. 1: $390 \mathrm{pp}$.
12. [ABRAMOVA, A.L.] АБРАМОВА А.Л. 1956. Indusiella thianschanica Broth. et C. Müll. (анатомо-морфологический очерк). - [Indusiella thianschanica Broth. et C. Müll., morphological overview] Бom. матер. Omd. cnop. pacm. Бom. uн-ma AH CCCP [Bot. Mat. Otd. Spor. Rast. Bot. Inst. Akad. Nauk SSSR] 11: 186-191.

13. [ABRAMOVA, A.L. \& I.I. ABRAMOV] АБРАМОВА А.Л., И.И. АБРАМОВ 1950. Несколько видов мхов новых для Кавказа. - [Some moss species new to Caucasus] Бот. матер. Отд. cnop. pacm. Бот. ин-та AH CCCP [Bot. Mat. Otd. Spor. Rast. Bot. Inst. Akad. Nauk SSSR] 6(7-12): 216-218.

14. [ABRAMOVA, A.L. \& I.I. ABRAMOV] АБРАМОВА А.Л., И.И. АБРАМОВ 1952. О некоторых редких видах мхов на Кавказе. - [On some rare species of mosses in the Caucasus] Бот. матер. Oтд. сnор. pacm. Бот. ин-та AH CCCP [Bot. Mat. Otd. Spor. Rast. Bot. Inst. Akad. Nauk SSSR] 8: 196-201.

15. [ABRAMOVA, A.L. \& I.I. ABRAMOV] АБРАMOBA А.Л., И.И. АБРАМОВ 1953. О новых и интересных видах мхов и печеночников с Кавказа. - [New and interesting species of mosses and hepatics in the Caucasus] Бот. матер. Omd. cnop. pacm. Бот. ин-ma AН СССР [Bot. Mat. Otd. Spor. Rast. Bot. Inst. Akad. Nauk SSSR] 9: 183-187.

16. [ABRAMOVA, A.L. \& I.I. ABRAMOV] АБРАМОВА А.Л., И.И. АБРАМОВ 1956. Hydrogonium Ehrenbergii (Lor.) Jaeg. из Средней Азии. - [Hydrogonium Ehrenbergii (Lor.) Jaeg. in the Middle Asia] Бom. мamep. Omd. cnop. pacm. Бот. uн-ma AH CCCP [Bot. Mat. Otd. Spor. Rast. Bot. Inst. Akad. Nauk SSSR] 11: 191-200.

17. [ABRAMOVA, A.L. \& I.I. ABRAMOV] АБРАМОВА А.Л., И.И. АБРАМОВ 1960. Новый род и новые виды мхов для ССCP: Bryobrittonia Williams - B. pellucida Williams и Hypnum subimponens Lesq. - [A new genus and new species of mosses for the USSR: Bryobrittonia Williams - B. pellucida Williams and Hypnum subimponens Lesq.] Бот. матер. Отд. сnор. pacm. Бот. ин-та AH CCCP [Bot. Mat. Otd. Spor. Rast. Bot. Inst. Akad. Nauk SSSR] 13: 294-305.

18. [ABRAMOVA, A.L. \& I.I. ABRAMOV] АБРАМОВА А.Л., И.И. АБРАМОВ 1962. О некоторых видах Кавказской бриофлоры. - [On some species of the Caucasian bryoflora] Бот. мamep. Oтд. cnop. pacm. Бот. uн-ma AH CCCP [Bot. Mat. Otd. Spor. Rast. Bot. Inst. Akad. Nauk SSSR] 15: 166-170.

19. [ABRAMOVA, A.L. \& I.I. ABRAMOV] АБРАМОВA А.Л., И.И. АБРАМОВ 1964. Мхи ущелья Кондара (Таджикская CCP). - [Mosses of Kondara Canyon (Tadjikskaya SSR)] Новости сист. низи. pacm. [Novosti Sist. Nizsh. Rast.] '1964': 340-341.

20. [ABRAMOVA, A.L. \& I.I. ABRAMOV] АБРАМОВА А.Л., И.И. АБРАМОВ 1966а. Редкие виды мхов Сибири и Дальнего Востока. - [Rare bryophytes of the Siberia and the Far East] Новости сист. низи. pacm. [Novosti Sist. Nizsh. Rast.] '1966': 302-315.

21. [ABRAMOVA, A.L. \& I.I. ABRAMOV] АБРАМОВА А.Л., И.И. АБРАМОВ 1966b. О мхах Молдавской ССР. 
- [On the bryophytes of Moldavian SSR] Новости сист. низи. pacm. [Novosti Sist. Nizsh. Rast.] '1966': 315-326.

22. [ABRAMOVA, A.L. \& I.I. ABRAMOV] АБРАMOBA А.Л., И.И. АБРАМОВ 1968. Новые и интересные виды бриофлоры CCCP. - [New and interesting species of the bryoflora of the USSR] Новости сист. низи. pacm. [Novosti Sist. Nizsh. Rast.] '1968': 298-302.

23. [ABRAMOVA, A.L. \& I.I. ABRAMOV] АБРАМОВА А.Л., И.И. АБРАМОВ 1969. Мхи. - [Bryophytes] $B$ кн.: Растительные сообщества и животное население степей и пустынь Центрального Казахстана (ред. Л.В. Арнольди, А. А. Юнатов) Л., Наука [In: Arnoldi, L. V. \& A. A. Yunatov (eds.), Rastitelnye soobshchestva i zhivotnoe naselenie stepei i pustyn tsentralnogo Kazakhstana. Leningrad, Nauka]: 291-295.

24. [ABRAMOVA, A.L. \& I.I. ABRAMOV] АБРАМОВА А.Л., И.И. АБРАМОВ 1969 [1970]. Новый мох для Дальнего Востока - Archidium alternifolium (Hedw.) Mitt. - [A new moss for the Far East - Archidium alternifolium (Hedw.) Mitt.] Новости сист. низи. pacm. [Novosti Sist. Nizsh. Rast.] 6: 262-265.

25. [ABRAMOVA, A.L. \& I.I. ABRAMOV] АБРАMOBA А.Л., И.И. АБРАМОВ 1970а. Об ареале Funaria aequidens Lindb. - [On the distribution of Funaria aequidens Lindb.] Новости сист. низи. pacm. [Novosti Sist. Nizsh. Rast.] 7: 342-347.

26. [ABRAMOVA, A.L. \& I.I. ABRAMOV] АБРАMOBA А.Л., И.И. АБРАМОВ 1970b. Сфагновые мхи в горах Средней Азии. - [Sphagna in the mountains of the Middle Asia] Новости сист. низи. pacm. [Novosti Sist. Nizsh. Rast.] 7: 333-342.

27. [ABRAMOVA, A.L. \& I.I. ABRAMOV] АБРАМОВА А.Л., И.И. АБРАМОВ 1972. Об изменчивости видов рода Dicranoweisia Lindb. в CCCP. - [On the variability of species of the genus Dicranoweisia Lindb. in the USSR] Hовости сист. низи. pacm. [Novosti Sist. Nizsh. Rast.] 9: 343-349.

28. [ABRAMOVA, A.L. \& I.I. ABRAMOV] АБРАМОВА А.Л., И.И. АБРАМОВ 1974. Обзор бриофлоры Кавказа. - [The outline of the bryoflora of the Caucasus] Новости cucm. низи. pacm. [Novosti Sist. Nizsh. Rast.] 11: 304-311.

29. [ABRAMOVA, A.L. \& I.I. ABRAMOV] AБPAMOBA А.Л., И.И. АБРАМОВ 1975. Hypnum plumaeforme Wils. на Кавказе. - [Hypnum plumaeforme Wils. in the Caucasus] Новости сист. низи. pacm. [Novosti Sist. Nizsh. Rast.] 12: 283-289.

30. [ABRAMOVA, A.L. \& I.I. ABRAMOV] АБРАМОВА А.Л., И.И. АБРАМОВ 1977. К бриофлоре Дальнего Востока. - [On the bryoflora of the Far East] Новости сист. низи. pacm. [Novosti Sist. Nizsh. Rast.] 14: 212-219.

31. [ABRAMOVA, A.L. \& I.I. ABRAMOV] АБРАMOBA А.Л., И.И. АБРАМОВ 1979. Редкие и интересные виды мхов с Кавказа. - [Rare and interesting species of mosses in the Caucasus] Новости сист. низш. pacm. [Novosti Sist. Nizsh. Rast.] 16: 158-160.

32. [ABRAMOVA, A.L. \& I.I. ABRAMOV] АБРАMOBA А.Л., И.И. АБРАМОВ 1983. Род Leptopterigynandrum C. Muell., новый для бриофлоры CCCP. - [Leptopteri- gynandrum C.Muell., a new genus for the bryoflora of the USSR] Новости сист. низи. pacm. [Novosti Sist. Nizsh. Rast.] 20: 161-168.

33. [ABRAMOVA, A.L. \& I.I. ABRAMOV] АБРАМОВА А.Л., И.И. АБРАМОВ 1985. К систематике рода Brotherella Loeske. - [On the taxonomy of the genus Brotherella Loeske] Новости сист. низи. pacm. [Novosti Sist. Nizsh. Rast.] 22: 206-210.

34. [ABRAMOVA, A.L. \& U.K.MAMATKULOV] АБРАМОВА А.Л., У.К. МАМАТКУЛОВ 1968. Интересные мхи из семейства Pottiaceae из Средней Азии. - [Interesting mosses of the family Pottiaceae from the Middle Asia] Докл. AH Taдж. CCP [Dokl. Akad. Nauk Tadj. SSR] 11(3): 53-56.

35. [ABRAMOVA, A.L., L.I. SAVICZ-LJUBITSKAYA \& Z.N. SMIRNOVA] АБРАMOВА А.Л., Л.И. САВИЧЛЮБИЦКАЯ, 3.Н. СМИРНОВА 1961. Определитель листостебельных мхов Арктики СССР. - [Handbook of mosses of Arctic of the USSR] Л., Изд-во АН СССР [Leningrad, Izd. Akad. Nauk SSSR], 716 pp.

36. [ABRAMOVA, A.L. \& N.A. STEPANOVA] АБРАМОВА А.Л., Н.А. СТЕПАНОВА 1986. К флоре мхов нижнего течения р. Колымы. - [On the moss flora of the lower course of Kolyma River] Новости сист. низш. раст. [Novosti Sist. Nizsh. Rast.] 23: 208-214.

37. [ABRAMOVA, L.I., L.V. BARDUNOV, I.A. GUBANOV, M.S. IGNATOV, S.N. KORENYUK, V.S. NOVIKOV, D.A. PETELIN, N.N. STEZURA] АБРАМОВА Л.И., Л.В. БАРДУНОВ, И.А. ГУБАНОВ, М.С. ИГНАТОВ, С.Н. КОРЕНЮК, В.С. НОВИКОВ, Д.А. ПЕТЕЛИН, Н.Н. СТЕЦУРА 1987. Мохообразные и сосудистые растения Зейского заповедника. - [Bryophytes and vascular plants of Zeyskiy Nature Reserve] Флора и фауна заповедников. Bun. 1. Москва [Flora i fauna zapovednikov. 1. Mochoobraznye $i$ sosudistye rasteniya Zeyskogo zapovednika. Moscow], $70 \mathrm{pp}$.

38. [AFONINA, O.M.] АФОНИНА О.М. 2000. Мохообразные. - [Bryophytes] Флора и фауна заповедников. Bыn. 88. Мохообразные и лишайники заповедника "Остров Врангеля». Москва [Flora i fauna zapovednikov. 88. Mochoobraznye i lishajniki zapovednika "Ostrov Vrangelya”. Moscow]: 6-46.

39. [AFONINA, O.M.] АФОНИНА О.М. 2002. Дополнения к флоре мхов архипелага Северная Земля. - [Additions to the moss flora of Severnaya Zemlya Archipelago] Новости сист. низи. pacm. [Novosti Sist. Nizch. Rast.] 36: $203-210$.

40. [AFONINA, O.M.] АФОНИНА О.М. 2004a. Конспект флоры мхов Чукотки. - [Moss flora of Chukotka] CПб, БИН РАН [Sankt-Petersburg, Bot. Inst. RAS], 260 pp.

41. [AFONINA, O.M.] АФОНИНА О.М. 2004b. Виды Нурnum секции Hamulosa (Musci, Hypnaceae) в России. [Hypnum sect. Hamulosa (Musci, Hypnaceae) in Russia] Arctoa 13: 9-28.

42. [AFONINA, O.M.] АФОНИНА О.М. 2006а. Мохообразные. - [Bryophyta] В кн.: Красная книга Ненечкого автономного округа (ред. Матвеева Н.В.), Нарьян-Мар [In: Matveeva, N.V. (ed.), Red Data Book of Nenetskij Autonomous District]: 118-125. 
43. AFONINA, O.M. 2006b. New moss records from Chukotskiy Autonomous District. 1. - Arctoa 15: 270.

44. AFONINA, O.M. 2006c. New moss records from Nenetskij Autonomous District. 1. - Arctoa 15: 251.

45. [AFONINA, O.M. \& H. ANDO] АФОНИНА O.M., Х. АНДО 2000. Hypnum holmenii (Musci, Hypnaceae) в бриофлоре России. - [Hypnum holmenii (Musci, Нypnaceae) in moss flora of Russia] Бот. Журн. [Bot. Zhurn.] 85(3): $40-46$

46. AFONINA, O.M. \& L.S. BLAGODATSKIKH 2006. New moss records from Magadan Province. 1. - Arctoa 15: 268.

47. AFONINA, O.M. \& I.V. CZERNYADJEVA 1995. Mosses of the Russian Arctic: check-list and bibliography. Arctoa 5: 99-142.

48. [AFONINA, O.M., E.A. IGNATOVA \& A.I. MAKSIMOV] АФОНИНА О.М., Е.А. ИГНАТОВА, А.И. МАКСИМОВ 2006. Stereodon fertilis (Pylaisiaceae, Musci) в России. Бот. журн. [Bot. Zhurn.] 91 (3): 329-335.

49. [AFONINA, O.M., T.M. KOROLEVA] АФОНИНА O.M., Т.М. КОРОЛЕВА 2006. Мхи острова Четырехстолбового (архипелаг Медвежьи Острова, Восточно-Сибирское мope). - [Mosses of the Chetyryokhstolbovoy Island (Medvezhy Islands Archipelago, the East-Siberian Sea] Новости cист. низи. pacm. [Novosti Sist. Nizsh. Rast.] 40.

50. [AFONINA, O.M., N.V. MATVEYEVA] АФОНИНА O.M., Н.В. МАТВЕЕВА 2004 Мхи острова Большевик (архипелаг Северная Земля). - [Mosses of the Bolshevik Island (Severnaya Zemlya archipelago)] Бот. журн. [Bot. Zhurn.] 88(9): 1-24.

51. [AFONINA, O.M. \& R. OCHYRA] АФОНИНА O.М., P. OХЫPA 1994. Schistidium cryptocarpum (Musci, Grimmiaceae) - новый вид для Евразии. - [Schistidium cryptocarpum (Musci, Grimmiaceae) - a species new to Eurasia] Бот. Журн. [Bot. Zhurn.] 79(10): 128-133.

52. [AFONINA, O.M., V.I. ZOLOTOV \& A.A.NOTOV] АФОНИНА О.М., В.И. ЗОЛОТОВ, А.А. НОТОВ 2006. К флоре мхов Оренбургского государственного природного заповедника. - [On the moss flora of Orenburgskiy State Nature Reserve] Степи Северной Евразии. Материаль IV международного симпозиума. Оренбург, ИПК «Газпромпечать» ООО «Оренбурггазпромсервис» [Stepi Severnoy Evrazii. Materialy IV mezhdunarodnogo simpoziuma. Orenburg, IPK "Gazprompechat", OOO “Orenburggazpromservis”]: 71-75.

53. AHTI, T. \& M. BOYCHUK 2006. The botanical journeys of A. K. Cajander and J. I. Lindroth to Karelia and Onega River in 1898 and 1899, with a list of their bryophyte and lichen collections. - Norrlinia 14: 1-65.

54. [AKATOV, V.V. \& T.V. AKATOVA] AKATOB B.B., T.B. АКАТОВА 2006. Высокогорный озерно-болотный комплекс реки Дзитаку (Западный Кавказ). - [High mountain mire-lake complex of Dzitaku River (West Caucasus)] В кн.: Водно-болотные угодья России. Т. 6. Водно-болотные угодья Северного Кавказа (ред. A.Л.Мищенко), M., Wetlands International [In: A.L. Mishchenko (ed.), Vodno-bolotnye ugodiya Rossii. T. 6. Vodno-bolotnye ugodiya Seevrnogo Kavkaza. Moscow, Wetlands International]: 126-129.
55. [AKATOVA, Т.V.] АКАТОВА Т.В. 2002. Листостебельные мхи Кавказского заповедника (Западный Кавказ, Россия). - [Moss flora of the Caucasian Nature Reserve (Western Caucasus, Russia)] Arctoa 11: 179-204.

56. AKATOVA, T.V. \& E.A. IGNATOVA 2000. Pogonatum neesii (Polytrichaceae, Musci) in the Russian Caucasus. Arctoa 9: 127-128.

57. AKATOVA, T.V., Z.Kh. KHARZINOV, E.A. IGNATOVA \& M.S. IGNATOV 2004. On three rare species of Orthotrichum (Orthotrichaceae, Musci) in Caucasus. Arctoa 13: 41-49.

58. [ALEKSENKO, M.A.] АЛЕКСЕНКО М.А. 1899. Бриологическая флора Литовского Полесья. - [Bryophyte flora of Litovskoye Polessie] Tp. O-ва исnыт. природы при Харьк. ун-те [Trudy Obshch. Ispyt. Prirody pri Kharkovsk. Univ.] 34: 91-136.

59. ALLEN, B. 1999. Data on species of Kazakhstan, Tadjikistan, Uzbekistan, Kyrgyzstan, Turkmenistan presented in MO database: http://mobot.mobot.org/cgi-bin/ search_vast

60. ANDERSON, L. E., H.A. CRUM \& W.R. BUCK 1990. List of mosses of North America north of Mexico. - Bryologist 93: 448-499.

61. [ANDREEVA, E.N.] АНДРЕEBA E.H. 2000. Новые виды водных листостебельных мхов Ленинградской области. - [New records of aquatic mosses for Leningrad Province] В кн.: Сохранение биологического разнообразия Фенноскандии. Международная конференция, Петрозаводск, 30 марта - 2 апреля, 2000 г. Петрозаводск [In: Conservation of biological diversity in Fennoscandia. Abstr. conf., Petrozavodsk, March 30 - April 2, 2000, Petrozavodsk]: 11 .

62. [ANDREEVA, E.N.] АНДРЕEBA Е.H. 2005. Мохообразные. - [Bryophytes] $B$ кн.: Юнтоловский региональный комплексный заказник (ред. Волкова Е.А. и др.). CПб [In: Volkova, E.A. et al., Yuntolovsky complex nature reserved area]: 123-132.

63. [ANDREEVA, E.N. \& L.E. KURBATOVA] АНДРЕЕВА Е.Н., Л.Е. КУРБАТОВА 2006. Мохообразные. - [Bryоphytes] В кн.: «Дудергофские высотыл - комплексный памятник природы (ред. Волкова Е.А. и др.). СПб [In: Volkova, E.A. et al., "Dudergoff heights" - complex nature protected area]: 68-85.

64. ARIKAVA, T. 2004. A taxonomic study of the genus Pylaisia (Hypnaceae, Musci). - J. Hattori Bot. Lab. 95: 71-154.

65. ARNELL, H.W. 1898. Musci novi. - Rev. Bryol. 25 (1): 1-9.

66. [BACHURINA, G.F. \& V.M. MEL'NICHUK] БАЧУРИНА Г.Ф., В.М. МЕЛЬНИЧУК 1987-1989. Флора мохів Украінськоі РСР. Андреєві, брієві. Вип. 1-3. [Moss flora of Ukrainian SSR. Andreaeopsida, Bryopsida. Pts. 1-3] Киів, Наукова Думка [Kyiv, Naukova Dumka]: 1 (1987), 180 pp.; 2 (1988), 180 pp.; 3 (1989) 175 pp. the fourth part of this book: [BACHURINA, G.F. \& V.M. MEL'NICHUK] БАЧУРИНА Г.Ф., В.М. МЕЛЬНИЧУК 2003. Флора мохів Украіни. Андреєві, брієві. Вип. 4. - [Moss flora of Ukraine. Andreaeopsida, Bryopsida. Pt. 
4] Киів, Національна Академія Наук Украіни [Куіv, Natsional'na Akademiya Nauk Ukraini], 256 pp.

67. BAKALIN, V.A. \& V.Ya. CHERDANTSEVA 2006. Bryophytes of northern Kuril Islands (North-West Pacific). Arctoa 15: 131-153.

68. [BARDUNOV, L.V.] БАРДУНОВ Л.В. 1960. Мхи гольцов Баргузинского хребта. - [Mosses of alpine belt of Barguzin Range] Тр. Баргузин. гос. зап-ка (УланУдэ), Bыn. 2 [Tr. Barguzin. Gos. Zap. (Ulan-Ude), part 2]: $135-145$.

69. [BARDUNOV, L.V.] БАРДУНОВ Л.В. 1969. Определитель листостебельных мхов Центральной Сибири. - [Handbook of mosses of the Central Siberia] Л., Наука [Leningrad, Nauka], 329 pp.

70. [BARDUNOV, L.V.] БАРДУНОВ Л.В. 1974. Листостебельные мхи Алтая и Саян. - [Mosses of the Altai and Sayan Mts.] Новосибирск, Наука [Novosibirsk, Nauka], $168 \mathrm{pp}$.

71. [BARDUNOV, L.V.] БАРДУНОВ Л.В. 1990а. Мохообразные. - [Mosses] В кн.: Уникальные объекты живой природы бассейна Байкала (ред. Попов Л. В.), Новосибирск, Наука [In: Popov, L. V. (ed.), Unikalnye ob'ekty zhivoj prirody basseina Baikala. Novosibirsk, Nauka]: 49-50.

72. [BARDUNOV, L.V.] БАРДУНОВ Л.В. 1990b. Восточноазиатские виды во флоре мхов Южной Сибири. [East-Asian species in moss flora of South Siberia] Бom. Журн. [Bot. Zhurn.] 75(5): 636-643.

73. [BARDUNOV, L.V.] БАРДУНОВ Л.В. 1992. Очерк бриофлоры Сибири. - [An overview of bryoflora of Siberia] Новосибирск, Наука [Novosibirsk, Nauka], 96 pp.

74. [BARDUNOV, L.V.] БАРДУНОВ Л.В. 2000. Материалы по флоре листостебельных мхов Витимского государственного заповедника. - [Materials on flora of mosses of Vitimsky State Reserve] Иркутск [Irkutsk], 35 pp.

75. [BARDUNOV, L.V. \& V.Ya. CHERDANTSEVA] БАРДУНОВ Л.В., В.Я. ЧЕРДАНЦЕВА 1978. Мохообразные. - [Bryophytes] В кн.:Флора и растительность Уссурийского заповедника. М., "Наука” [In: Flora i rastitel'nost' Ussurijskogo zapovednika. Moscow, "Nauka"]: 127-148.

76. [BARDUNOV, L.V. \& V.Ya. CHERDANTSEVA] БАРДУНОВ Л.В., В.Я. ЧЕРДАНЦЕВА 1982. Листостебельные мхи Южного Приморья. - [Mosses of the South of Primorskij Province] Новосибирск, Наука [Novosibirsk, Nauka], $208 \mathrm{pp}$.

77. [BARDUNOV, L.V. \& V.Ya. CHERDANTSEVA] БАРДУНОВ Л.В., В.Я. ЧЕРДАНЦЕВА 1984. Материалы по флоре листостебельных мхов Южных Курильских островов. - [Contributions to the moss flora of the southern Kuril Islands] В кн.: Систематикофлористические исследования флоры споровых растений Дальнего Востока (ред. Черданиева В.Я.). Владивосток, ДВНЦ АН ССCP [In: Cherdantseva V.Ya. (ed.), Sistematiko-floristicheskie issledovaniya flory sporovykh rastenij Dal'nego Vostoka. Vladivostok, Dal'nevost. Nauchn. Tsentr Akad. Nauk SSSR]: 34-53.

78. [BARDUNOV, L.V. \& V.Ya. CHERDANTSEVA] БАРДУНОВ Л.В., В.Я. ЧЕРДАНЦЕВА 2006. Мохо- образные. - [Bryophytes] В кн.: Флора, растительность и микобиота заповедника «Уссурийский» (ред. Васильева Лар.Н.). Владивосток, Дальнаука [In: Vasilieva, Lar. $N$. (ed.), Flora, rastitel'nost' $i$ micobiota of the reserve "Ussurijsky". Vladivostok, Dal'nauka]: 51-78.

79. [BARDUNOV, L.V., S.K. GAMBARYAN \& V.YA. CHERDANTSEVA] БАРДУНОВ Л.В., С.К. ГАМБАРЯН, В.Я. ЧЕРДАНЦЕВА 2002. Мохообразные. [Bryophytes] В кн.: Флора, микобиота и растительность Лазовского заповедника (ред. Егорова Л.Н.), Владивосток, Изд-во Русский Остров [In: Egorova, L.N. (ed.), Flora, mycobiota i rastitel'nost' Lazovskogo zapovednika. Vladivostok, «Pusskij Ostrov»]: 49-67.

80. BEDNAREK-OCHYRA, H. 1993. The taxonomic status of Racomitrium canescens fo. acicularioides (Musci, Grimmiaceae). - Fragm. Florist. et Geobot. 38 (2):741-743.

81. BEDNAREK-OCHYRA, H. 2006. A taxonomic monograph of the moss genus Codriophorus P. Beauv. (Grimmiaceae). - Krakow, W.Szafer Inst. of Botany, Polish Acad. Sci., $276 \mathrm{pp}$.

82. BELKINA, О.А.] БЕЛКИНА О.А. 2001. Находка Теtrodontium repandum (Funck) Schwaegr. (Musci) в России. - [New record of Tetrodontium repandum (Funck) Schwaegr. (Musci) in Russia] Arctoa 10: 71-74.

83. [BELKINA, О.А.] БЕЛКИНА О.А. 2003. Андреа Блютта. - [Andreaea blyttii Schimp.] В кн.: Красная Книга Мурманской области (ред. Н.А.Константинова и др.). Мурманск, Мурманское книжное издательство [In: Konstantinova, N.A. \& al., (eds.), Red Data book of the Murmansk Province. Murmansk, Murmanskoe Knizhnoe Izdatelstvo]: 133-134.

84. [BELKINA, O.A, N.A. KONSTANTINOVA \& V.A. KOSTINА] БЕЛКИНА О.А., Н.А. КОНСТАНТИНОВА, В.А. КОСТИНА 1991. Флора высших растений Ловозерских гор. - [The flora of higher plants of Lovozerskiye Mountains] СПб., Наука. [St.-Petersburg, Nauka], 206 pp.

85. [BELKINA, O.A. \& A.Yu. LIKHACHEV] БЕЛКИНА О.А., А.Ю. ЛИХАЧЕВ 1999. Некоторые особенности флоры листостебельных мхов Кандалакшского заповедника (Белое море). - [Some peculiarities of the moss flora of Kandalaksha State Reserve (White Sea, North-West Russia)] Бот. Журн. [Bot. Zhurn.] 84(11): 36-49.

86. [BELKINA, O.A. \& A.YU. LIKHACHEV] БЕЛКИНА О.А., А.Ю. ЛИХАЧЕВ 2004. Флора листостебельных мхов горных массивов Чильтальд и Ионн-Ньюгоайв (Мурманская область). - [Moss flora of mountains Chiltald and Ionn-Njugoayv (Murmansk Province)] Arctoa 13: 211-222.

87. [BELKINA, O.A. \& A.Yu. LIKHACHEV] БЕЛКИНА О.А., А.Ю. ЛИХАЧЕВ 2005. Флора листостебельных мхов Сальных тундр (Мурманская область). - [Moss flora of the Salnye tundry (Murmansk Province)] Arctoa 14: 177-196.

88. [BEZGODOV, A.G.] БЕЗГОДОВ А.Г. 2002. К бриофлоре окрестностей Кунгура (Пермская область). - [On the bryoflora of the Kungur City environs (Perm Province)] Arctoa 11: 53-62.

89. [BEZGODOV, A.G.] БЕЗГОДОВ А.Г. 2000. Мхи города Перми (Средний Урал). - [Mosses of the Perm City (Middle Ural, Russia)] Arctoa 9: 141-150. 
90. [BEZGODOV, A.G., I.L. GOLDBERG, M.V. DULIN, T.P. SHUBINA \& I.B. KUCHEROV] БЕЗГОДОВ А.Г., И.Л. ГОЛЬДБЕРГ, М.В. ДУЛИН, Т.П. ШУБИНА, И.Б. КУЧЕРОВ 2003. Дополнения к бриофлоре ПечороИлычского биосферного заповедника (Северный Урал). - [Additions to the bryoflora of the Pechora-Ilych biosphere reserve (Northern Urals)] Arctoa 12: 169-178.

91. [BEZGODOV A.G., E.A. IGNATOVA \& M.S. IGNATOV] БЕЗГОДОВ А.Г., Е.А. ИГНАТОВА, М.С. ИГНАТОВ 2006. Новые находки мхов в Пермской области. 1. [New moss records from Perm Province. 1] Arctoa 15: 253-254.

92. [BLAGODATSKIKH, L.S.] БЛАГОДАТСКИХ Л.С. 1972. К бриофлоре Западного Таймыра. - [On bryoflora of the Western Taimyr] Новости сист. низи. раст. [Novosti Sist. Nizsh. Rast.] 9: 358-364.

93. [BLAGODATSKIKH, L.S.] БЛАГОДАТСКИХ Л.С. 1973. Новые и редкие виды мхов для Таймырского полуострова. - [New and rare mosses for the Taimyr Peninsula] Новости сист. низи. pacm. [Novosti Sist. Nizsh. Rast.] 10: 325-332.

94. [BLAGODATSKIKH, L.S.] БЛАГОДАТСКИХ Л.С. 1984. Листостебельные мхи Колымского нагорья. - [Mosses of Kolyma Upland] Магадан, Ин-т Биолю Пробл. Севера [Magadan, Inst. Biol. Problem Severa], 47 pp.

95. [BLAGODATSKIKH, L.S.] БЛАГОДАТСКИХ Л.С. 1989. Редкие виды листостебельных мхов Колымского нагорья. - [Rare mosses of Kolyma Upland] $B \kappa н$.: Проблемы бриологии (ред. И.И.Абрамов). Л., Наука [In: Abramov, I.I. (ed.), Problemy Briologii]: 43-48.

96. [BLAGODATSKIKH, L.S., A.L.ZHUKOVA \& N.V. MATVЕҮЕVА] БЛАГОДАТСКИХ Л.С., А.Л. ЖУКОВА, Н.В. МАТВЕЕВА 1979. К флоре листостебельных и печеночных мхов окрестностей бухты Марии Прончищевой (Северо-Восточный Таймыр). - [On flora of mosses and hepatics of vicinity of Maria Pronchishcheva Bay (North-Eastern Taimyr)] В кн.: Арктические тундры и полярные пустыни Таймыра. Л.: Наука [In: Arkticheskie tundry i polyarnye pustyni Taimyra. Leningrad, Nauka]: 133-139.

97. BLOM, H.H. 1996. A revision of the Schistidium apocarpum complex in Norway and Sweden.-Bryoph. Bibl. 49: $1-333$.

98. BLOM, H.H. 1998. Genus Schistidum. - In.: Nyholm, E. Illustrated flora of Nordic mosses, Fasc. 4. Aulacomniaceae-Meesiaceae - Catoscopiaceae - Bartramiaceae Timmiaceae - Encalyptaceae - Grimmiaceae - Ptychomitraceae - Hedwigiaceae - Orthotrichaceae. Copenhagen \& Lund: Nordic Bryological Society: 287-330.

99. BLOM, H.H., E.A. IGNATOVA \& O.M. AFONINA 2006. New records of Schistidium (Grimmiaceae, Musci) in Russia. - Arctoa 15: 187-194.

100. [ВОYСНUK, М.А.] БОЙЧУК М.А. 2001. К флоре листостебельных мхов заповедника Костомукшский и окрестностей города Костомукша (Карелия). - [On moss flora of Kostomukshsky Nature Reserve and Kostomuksha vicinity (Karelia)] Новости сист. низи. pacm. [Novosti Sist. Nizch. Rast.] 35: 217-229.
101. BOYCHUK, M.A. \& T. AHTI 2005. Bryological results of the expeditions by A. K. Cajander and J. I. Lindroth to Karelia and Onega River in 1898-1899. - В кн.: «Актуальные проблемы бриологии», Тр. междунар. сов. посв. 90-летию со дня рожд. А.Л. Абрамовой. СПб, 2225 ноября 2005. [In: Proc. Int. Conf. "Actual Problems of Bryology" devoted to $90^{\text {th }}$ Anniversary of A.L. Abramova. St. Petersburg, 22-25 November 2005]: 33-37.

102. BROTHERUS, V.F. 1892. Enumeratio muscorum Caucasi. - Acta Soc. Sci. Fenn. 19(12): 1-170.

103. BROTHERUS, V.F. 1898. Indusiella, eine neue Laubmoos Gattung aus Central-Asiaen. - Bot. Centralbl. 75 (11): $1-2$.

104. [BROTHERUS, V.F.] БРОТЕРУC В.Ф. 1914. Мхи (Andreaeales; Bryales, часть 1-ая). - [Mosses (Andreaeales; Bryales, part 1)] В кн.: Федченко, Б.А. Флора Азіатской Россіи. Вып. 4. Петроград, Переселенческое управление [In: Fedchenko, B.A. Flora Aziatskoj Rossii, Pt. 4. Petrograd, Pereselencheskoe Upravlenie], 1-78.

105. [BROTHERUS, V.F.] БРОТЕРУC В.Ф. 1918. Мхи (Bryales, часть 2-ая). - [Mosses (Bryales, part 2)] В кн.: Федченко, Б.А. Флора Азіатской Россіи. Bbln. 13. Петроград, Переселенческое управление [In: Fedchenko, B.A. Flora Aziatskoj Rossii, Pt. 13. Petrograd, Pereselencheskoe Upravlenie], 79-182.

106. [BROTHERUS, V.F.] БРОТЕРУC В.Ф. 1965. Новые виды мхов из Азиатской части СCCP. - [New species of mosses from the Asian Russia] Новости сист.низи.раст. [Novosti Sist. Nizsh. Rast.] '1965': 273-277.

107. BRUMMITT, R.K. \& C.E. POWELL 1992. Authors of plant names. - Kew, Royal Botanic Gardens.

108. BURLEY, J.S. \& N.M. PRITCHARD 1990. Revision of the genus Ceratodon (Bryophyta). - Harvard Papers in Botany 2: 17-76.

109. [CHERDANTSEVA, V.Ya.] ЧЕРДАНЦЕВА В.Я. 1976. Конспект флоры листостебельных мхов Южного Сахалина. - [Conspectus of mosses of South Sakhalin] $B$ кн.: Низшие растения Дальнего Востока (ред. Л.Н.Васильева, М.М.Назарова), ДВО, БПИ [In: Vasilieva, L.N. \& M.M.Nazarova (eds.), Nizshie rasteniya Dalnego Vostoka. Dal'nevostochyi Nauchyi Centr, BiologoPochvennyi Institut]: 140-177.

110. [CHERDANTSEVA, V.Ya.] ЧЕРДАНЦЕВА В.Я. 1989. Редкие и интересные виды мхов Дальнего Востока CCCP. - [Rare and interesting moss species for the Far East of the USSR] Новости сист. низи. pacm. [Novosti Sist. Nizsh. Rast.] 26: 157-159.

111. [CHERDANTSEVA, V.Ya.] ЧЕРДАНЦЕВА В.Я. 2002 [2003]. Листостебельные мхи Сихотэ-Алинского биосферного заповедника (Дальний Восток, Приморский край). - [Mosses of the Sikhote-Alinsky Biosphere Reserve (Russian Far East, Primorsky Territory)] Arctoa 11: $229-244$.

112. [CHERDANTSEVA, V.Ya.] ЧЕРДАНЦЕВА В.Я. 2006. Листостебельные мхи (Bryopsida) острова Монерон. [Mosses (Bryopsida) of Moneron Island] В кн.: Растительный и животный мир острова Монерон (Материаль Международного Сахалинского проекта). 
Владивосток, Дальнаука [In: Rastitelnyj i zhivotnyj mir ostova Moneron (Materialy Mezhdunarodnogo Sakhalinskogo Projekta), Vladivostok, Dal'nauka]: 48-54.

113. [CHERDANTSEVA, V.Ya. \& S.K. GAMBARYAN] ЧЕРДАНЦЕВА В.Я., С.К. ГАМБАРЯН 1986. Мохообразные. - [Bryophytes] В кн.: Флора и растительность Большехехиирского заповедника. Владивосток [In: Flora i rastitelnost' Bolshekhekhzirskogo zapovednika. Vladivostok]: 79-101.

114. CHERDANTSEVA, V.YA., K.V. GOROBETS, J. HARPEL, M.S. IGNATOV, E.A. IGNATOVA, V.V. TELEGANOVA 2006. New moss records from Sakhalinskaya Province. 1. Sakhalin. - Arctoa 15: 263-268.

115. CHERDANTSEVA, V.YA., M.S. IGNATOV \& E.A. IGNATOVA 2006a. New moss records from Sakhalinskaya Province. 2. Kunashir. - Arctoa 15: 268.

116. CHERDANTSEVA, V.YA., M.S. IGNATOV \& E.A. IGNATOVA 2006b. New moss records from Primorsky Territory. 1. - Arctoa 15: 263.

117. CHIKOVANI, N., T. SVANIDZE 2004. Checklist of bryophyte species of Georgia. - Braun-Blanquetia 34: 97-116.

118. [CHURAKOVA, E.N.] ЧУРАКОВА E.H. 2002. Листостебельные мхи таёжной зоны Архангельской области. - [Mosses of the taiga zone of the Arkhangelsk Province (northern European Russia)] Arctoa 11: 351-392.

119. CHURCHILL, S.P. 1987. Systematics and biogeography of Jaffueliobryum (Grimmiaceae). -Mem. New York Bot. Gard. 45: 691-708.

120. CORLEY, M.F.V. \& A.C. CRUNDWELL 1991. Additions and amendments to the mosses of Europe and the Azores. - J. Bryol. 16: 337-356.

121. CORLEY, M.F.V., A.C. CRUNDWELL, R. DÜLL, M.O. HILL \& A.J.E. SMITH 1981. Mosses of Europe and the Azores: an annotated list of species, with synonyms from the recent literature. - J. Bryol. 11: 609-689.

122. CRUM, H.A. \& L.E. ANDERSON 1981. Mosses of Eastern North America (Vol. 1-2). - New York, Colombia University Press, $1328 \mathrm{pp}$.

123. CRUM, H., W.C. STEERE \& L.E. ANDERSON 1979. A new list of mosses of North America north of Mexico. Bryologist 76: 85-130.

124. CRUNDWELL, A.C., E. NYHOLM 1962. A study of Campylium hispidulum and related species. - Trans. Brit. Bryol. Soc. 4: 194-200.

125. CRUNDWELL, A.C. \& E. NYHOLM 1974. Funaria muhlenbergii and related European species. - Lindbergia 2: $222-229$.

126. [CZERNYADJEVA, I.V.] ЧЕРНЯДЬЕВА И.В. 1992. Анализ активности видов бриофлоры северо-запада плато Путорана. - [Analysis of species activity in bryoflora of North-West of Putorana Plateau] Новости Cucm. Низи. Pacm. [Novosti Sist. Nizsh. Rast.] 28: 161-165.

127. [CZERNYADJEVA, I.V.] ЧЕРНЯДЬЕВА И.В. 1994a. Флора листостебельных мхов окрестностей стационара “Собь” (Полярный Урал). - [The moss flora of the region of Sob Station (Polar Ural)] Arctoa 3: 133-138.

128. [CZERNYADJEVA, I.V.] ЧЕРНЯДЬЕВА И.В. 1994 b.
Листостебельные мхи низовьев реки Чугорьяха (Югозападная часть Гыданского полуострова, Западносибирская Арктика). - [Mosses of the lower reaches of the Chugor'yakha River (south-western part of the Gydan Peninsula, West Siberian Arctic)] Бот. Журн. [Bot. Zhurn.] 79(8): 57-67.

129. CZERNYADJEVA, I.V. 1995a. Philonotis yezoana Besch. et Card. ex Card. (Bartramiaceae, Musci) new to Russia. - Arctoa 4: 15-16.

130. CZERNYADJEVA, I.V. 1995b. Pohlia cardotii (Bryaceae, Musci) found in Eurasia (Russia, Kamtchatka Peninsula). - Ann. Bot. Fennici 32: 137-139.

131. [CZERNYADJEVA, I.V.] ЧЕРНЯДЬЕВА И.В. 1997. Виды рода Pohlia (Musci) с выводковыми почками. [The species of the genus Pohlia (Musci) with propagula] Бот. Журн. [Bot. Zhurn.] 82(1): 102-122.

132. CZERNYADJEVA, I.V. 1999a. On the distribution of propaguliferous species of Pohlia (Bryaceae, Musci) in Russia. - Arctoa 8: 51-56.

133. CZERNYADJEVA, I.V. 1999b. New record of Dicranella riparia (H. Lindb.) Mårt. et Nyh. (Dicranaceae, Musci) in Russia. - Arctoa 8: 71-72.

134. CZERNYADJEVA, I.V. 2000. Fissidens arcticus Bryhn in Russia. - Arctoa 9: 25-28.

135. [CZERNYADJEVA, I.V.] ЧЕРНЯДЬЕВА, И.В. 2001a. Листостебельные мхи заповедника Большая Кокшага (Республика Марий Эл). - [Mosses of Bol'shaya Kokshaga Reserve (Mari El Republic, European Part Russia] Hовости сист. низи. pacm. [Novosti Sist. Nizch. Rast.] 35: $266-278$.

136. CZERNYADJEVA, I.V. 2001b. Moss flora of Yamal Peninsula (West Siberian Arctic). - Arctoa 10: 121-150.

137. CZERNYADJEVA, I.V. 2002. Dichelyma capillaceum (Dicks.) Myr. (Musci) in Russia - Arctoa 11: 87-89.

138. [CZERNYADJEVA, I.V.] ЧЕРНЯДЬЕВА И.В. 2003 [2004]. Род Hygrohypnum (Amblystegiaceae, Musci) в России. - [The genus Hygrohypnum (Amblystegiaceae, Musci) in Russia] Arctoa 12: 25-58.

139. CZERNYADJEVA, I.V. 2004. Anacamptodon kamchaticum, a new species of Amblystegiaceae (Musci) from Far East. - Arctoa 13: 5-8.

140. CZERNYADJEVA, I.V. 2005. A check-list of the mosses of Kamchatka Peninsula (Far East). - Arctoa 14: 13-34.

141. [CZERNYADJEVA, I.V.] ЧЕРНЯДЬЕВА, И. В. 2006. Новые находки мхов в Камчатской области. 1. - [New moss records from Kamchatskaya Province. 1] Arctoa 15: $268-270$

142. CZERNYADJEVA, I.V., V.YA. CHERDANTSEVA, M.S. IGNATOV \& I.A. MILYUTINA 2006. Thuidium thermophilum (Thuidiaceae, Bryophyta), a new species from Kamchatka. - Arctoa 15: 195-202.

143. CZERNYADJEVA, I. V. \& M.S IGNATOV 2006. The first record of Sciuro-hypnum uncinifolium (Brachytheciaceae, Musci) in Russia. - J.Hattori Bot. Lab. 99: 271-274.

144. CZERNYADJEVA, I.V. \& E.A IGNATOVA 2004. Pohlia tundrae Shaw (Bryaceae, Musci) in Russia. - Arctoa 13: 29-32. 
145. [CZERNYADJEVA, I.V. \& L.E. KURBATOVA] ЧЕРНЯДЬЕВА И.В., Л.Е. КУРБАТОВА 1995.К флоре листостебельных мхов долины реки Поной (Кольский полуостров). - [On the flora of mosses of the River Ponoy (Kola Peninsula)] Вестн. СПбГУ. Сер. 3, вылn. 2 [Vestnik Sankt-Peterburg. Univ., Ser. 3, vyp. 2] 10: 56-62.

146. [CZERNYADJEVA, I.V. \& E.Yu. KUZMINA] ЧЕPНЯДЬЕВА И.В., Е.Ю. КУЗЬМИНА 2002. Мхи окрестностей города Сургут (Западная Сибирь). - [Mosses of Surgut environs (Western Siberia)] Новости сист. низи. pacm. [Novosti Sist. Nizsh. Rast.] 14: 254-269.

147. [CZERNYADJEVA, I.V. \& A.D. POTEMKIN] ЧEPНЯДЬЕВА, И.В., А.Д. ПОТЕМКИН 2002. Флора мохообразных заповедника. - [The bryoflora of the Reserve] В кн.: Растительность, флора и почвы ВерхнеТазовского Государственного заповедника (ред. В.Ю. Hешатаев). C.-Петербург [In: V.Yu. Neshatayev. Vegetation, flora and soils of the Verhne-Tazovskii State Reserve. Sankt-Petersburg]: 35-46.

148. [DANILKIV, I.S., O.V. LOBOCHESKA, Z.I. MAMCHUR \& M.I. SOROKA] ДАНИЛКIB I.C., O.B. ЛОБАЧЕВСЬКА, 3.I. МАМЧУР, М.I. СОРОКА 2002. Мохоподібні Украінського Розточчя. - [Bryophytes of Ukrainian Roztochchya] Львів, Iнст. Екол. Kapnam [Lviv, Inst. Ekol. Karpat], 320 pp.

149. [DRUGOVA, Т.Р.] ДРУГОВА Т.П. 2005. Флора листостебельных мхов г. Кировска (Мурманская область, север европейской России). - [Moss flora of Kirovsk Town, north of European Russia] Arctoa 14: 203-209.

150. [DYACHENKO, А.Р.] ДЬЯЧЕНКО А.П. 1997. Флора листостебельных мхов Урала. Ч. 1. - [The moss flora of the Urals. Pt. 1]. Екатеринбург, Изд-во Уральск. ун-та [Ekaterinburg, Izd-vo Uralsk. Univ.]: 264.

151. [DYACHENKO, А.Р.] ДЬЯЧЕНКО А.П. 2001. Bryum stirtonii Bruch et Schimp. in Bruch et al. (сем. Bryaceae Schwaegr. in Willd.) и Pseudocalliergon trifarium (Web. et Mohr) Loeske (сем. Amblystegiaceae G. Roth) - новые для Урала виды мхов (кл. Musci). - [Bryum stirtonii Bruch et Schimp. in Bruch et al. (сем. Bryaceae Schwaegr. in Willd.) and Pseudocalliergon trifarium (Web. et Mohr) Loeske (Amblystegiaceae G. Roth) - new species of mosses (Musci) for Urals] В кн.: Флора и растительность Сибири и Дальнего Востока. Чтения памяти Л..М. Черепнина: Материаль III Российской конф. Красноярск: КГПУ. [In: Flora i rastitel'nost' Sibiri i Dal'nego Vostoka. Chteniya pamyati L.M. Cherepnina: Materialy III Rossijskoj konferencii. Krasnoyarsk: KGPU] : 65-66.

152. [DYACHENKO, A.P., M.N. BYSTRUSHKINA, A.G. BYSTRUSHKIN \& N.A. STAFEEVA] ДЬЯЧЕНКО А.П., М.Н. БЫСТРУШКИНА, А.Г. БЫСТРУШКИН, Н.А. СТАФЕЕВА 2005. К флоре мхов национального парка «Таганай» (Южный Урал). - [On moss flora of National Park "Taganay" (South Urals)] В кн.: Исследования природных и социально-экономических систем Урала. Екатеринбур [In: Issledovaniya prirodnykh i sozialnoeconomicheskikh sistem Urala, Ekaterinburg]: 5-14.

153. [DYLEVSKAYA, I.V.] ДЫЛЕВСКАЯ И.В. 1963. Leptobarbula Schimp. - новый род для CCCP. - [Leptobarbula Schimp. - new genus for the USSR] Бот. матер.
Omd. cnop. pacm. Бот. ин-ma AH CCCP [Bot. Mat. Otd. Spor. Rast. Bot. Inst. Akad. Nauk SSSR] 16: 199-202.

154. DÜLL, R. 1984-1985. Distribution of the European and Macaronesian mosses (Bryophytina), Pts. 1-2. - Bryol. Beitr. Pt. 1 (1984) 4: 1-109; Pt. 2 (1985) 5: 110-232.

155. [EREMINA, N.Kh.] ЕРЕМИНА Н.Х. 1964. Новые виды для бриофлоры СCCP - Funaria attenuata (Dicks.) Lindb. и Orthotrichum laevigatum Zett. - [New species for bryoflora of the USSR - Funaria attenuata (Dicks.) Lindb. and Orthotrichum laevigatum Zett.] Новости сист. низи. pacm. [Novosti Sist. Nizsh. Rast.] '1964': 318-324.

156. [EREMINA, N.Kh.] ЕРЕМИНА Н.Х. 1965a. Материалы к бриофлоре северного Тянь-Шаня - [Materials on bryoflora of Northern Tian-Shan] В кн.: Споровые растения Средней Азии и Казахстана. Ташкент [In: Sporovye raseniya Srednej Azii i Kazakhstana. Tashkent]: 209-213.

157. [EREMINA, N.Kh.] ЕРЕМИНА Н.X. 1965b. Материалы к флоре акрокарпных мхов Заилийского Алатау. - [Materials on flora of acrocarpous mosses of Zailiisky Alatau] Бот. мат. Гербария ин-та ботаники АН Каз ССР [Bot. Mat. Gerb. Inst. Bot. Akad. Nauk Kazakhskoj SSR] 3: 115-125.

158. [EREMINA, N.Kh.] ЕРЕМИНА Н.Х. 1966. Поясное распределение некоторых видов мхов в Заилийском Алатау. - [Altitudinal distribution of some moss species in Zailiisky Alatau] Бот. мат. Гербария ин-та ботаники AH Каз CCP [Bot. Mat. Gerb. Inst. Bot. Akad. Nauk Kazakhskoj SSRJ 4: 155-161.

159. [EREMINA, N.Kh., U.Kh. SUYUNSHALIEVA \& L.N. PRUS] ЕРЕМИНА Н.Х., У.Х. СУЮНШАЛИЕВА, Л.Н. ПРУС 1970. К бриофлоре Алма-Атинского государственного заповедника. - [On bryoflora of Alma-Atinsky State Reserve] Труды Алма-Атинского гос. заповедн. [Trudy Alma-Atinskogo Gos. Zapov.] 9: 46-50.

160. [EREMINA, N.Kh. \& T.P. YURCHENKO] ЕРЕМИНA Н.Х., Т.П. ЮРЧЕНКО 1968 [1970]. К бриофлоре ущелья Большой Киргизсай Кетменского хребта. - [On bryoflora of Bolshoj Kyrgyzsaj Canyon of Ketmensky Range] Биология и география [Biologia i Geografia] 5: 26-29.

161. [FEDOSOV, V.E.] ФЕДОСОВ В.Э. 2006а. Новые находки мхов в Камчатской области. 2. - [New moss records from Kamchatskaya Province. 2] Arctoa 15: 270.

162. [FEDOSOV, V.E.] ФЕДОСОВ В.Э. 2006b. Новые находки мхов в Таймырском автономном округе. 1. [New moss records from Taimyr Autonomous District. 1] Arctoa 15: 258-260.

163. [FEDOSOV, V.E.] ФЕДОСОВ В.Э. 2006c. Мхи Анабарского плато (рукопись, сборы MW). - Mosses of Anabar Plateau (manuscript, collections in MW).

164. FEDOSOV, V.E. \& E.A. IGNATOVA 2005a. Bryophyte flora of the "Ledyanaya Bay" Key plot (Byrranga Range, Taimyr, Siberian Arctic). - Arctoa 14: 71-94.

165. FEDOSOV, V.E. \& E.A. IGNATOVA 2005b. The genus Pseudocrossidium (Pottiaceae, Musci) in Russia. - Arctoa 15: 203-210.

166. [FEDOSOV, V. Е. \& E.A.IGNATOVA.] ФЕДОСОВ В.Э., Е.А. ИГНАТОВА 2006. Новые находки мхов в республике Коми. 2. - [New moss records from Komi Republic. 2] Arctoa 15: 252-253. 
167. [FEDOSOV, V.E. \& S.YU. POPOV] ФЕДОСОВ В. Э., С.Ю. ПОПОВ 2006. Новые находки мхов в Красноярском крае. 1. - [New moss records from Krasnoyarsk Territory. 1] Arctoa 15: 260-261.

168. FLATBERG, K.I. 1984. A taxonomic revision of the Sphagnum imbricatum complex. - Det K. Norske Vidensk. Selsk. Skrift. '1984'(3): 1-80.

169. FLATBERG, K.F. 1988. Taxonomy of Sphagnum annulatum and related species. - Ann. Bot. Fennici 25(4): 303-350.

170. FLATBERG, K.I. 2005. Taxonomy, geography and possible origin of Sphagnum inexspectatum (sect. Subsecunda) sp. nov. - Lindbergia 30: 59-78.

171. FRISVOLL, A.A. 1985. Lectotypifications including nomenclatural and taxonomical notes on Ditrichum flexicaule sensu lato. - Bryologist 88: 31-40

172. FRISVOLL, A.A. 1988. A taxonomic revision of the Racomitrium heterostichum group (Bryophyta, Grimmiales) in N. and C. America, N. Africa, Europe and Asia. Gunneria 59: 5-289.

173. GALLEGO, M.T. 2005. A taxonomic study of the genus Syntrichia Brid. (Pottiaceae, Musci) in the Mediterranean region and Macaronesia. - J. Hattori Bot. Lab. 98: 47-122.

174. [GAMBARYAN, S.K. \& V.Ya. CHERDANTSEVA] ГАМБАРЯН С.К., В.Я. ЧЕРДАНЦЕВА 2002. Мохообразные. - [Bryophytes] В кн.: Кадастр растений и грибов заповедника "Кедровая падь". Владивосток [In.: Kadastr rastenii i gribov zapovednika "Kedrovaya pad". Vladivostok]: 21-30.

175. GOFFINET, B. 2002. Orthotrichum sprucei Mont. (Musci), a European endemic discovered in Kazakhstan - Arctoa 11: $27-30$.

176. GOFFINET, B. \& W.R. BUCK 2004. Systematics of the Bryophyta (mosses): from molecules to a revised classification. - Monographs in Systematic Botany from the Missouri Botanical Garden 98: 205-239.

177. GOLDBERG, I.L. 2002. The saxicolous moss flora of the Middle Urals. - Arctoa 11: 63-80.

178. [GOROBETS, K.V.] ГОРОБЕЦ К.В. 2004. Флора листостебельных мхов п-ова Муравьева-Амурского и островов Залива Петра Великого (Приморский край). - [Moss flora of Muravjov-Amursky's Peninsula and islands of the Petra Velikogo Bay] Автореф. дисс... канд. биол. наук. Владивосток, ТИБОХ ДВО РАН [Thesis Ph. D.. Vladivosok, Tikhookeansky Inst. Bioorg. Chimii Dalnevost. Otd. Ross. Akad. Nauk], 22 pp.

179. GRANZOW-DE LA CERDA, I. 1997. Revision and phylogenetic study of Anomodon and Herpetineuron (Anomodontaceae, Musci). - Contr. Univ. Michigan Herb. 21: 205-275.

180. GREVEN, H.C. 1995. Grimmia Hedw. (Grimmiaceae, Musci) in Europe. - Leiden: Backhuys Publishers. 160 pp.

181. HE, S. 1997. A revision of Homalia (Musci: Neckeraceae). - J. Hattori Bot. Lab. 81: 1-52.

182. HE, S. 2005. A revision of the genus Leptopterigynandrum (Bryopsida, Leskeaceae). - J. Hattori Bot. Lab. 95: 71-154.

183. HEDENÄS, L. 1989. Amblystegium longicuspis Lindb. \& H. Arn., its status and taxonimic position. -
Lindbergia 14: 142-146.

184. HEDENÄS, L. 1989. The genus Sanionia (Musci) in northwestern Europe, a taxonomic revision. - Ann. Bot. Fenn. 26: 399-416.

185. HEDENÄS, L. 1990. Taxonomic and nomenclatural notes on the genera Calliergonella and Breidleria. - Lindbergia 16: 161-168.

186. HEDENÄS, L. 1996. On the identity of Brachythecium campestre (C. Mull.) B., S. \& G. in Sweden, Norway and Finland. - Lindbergia 20: 94-101.

187. HEDENÄS, L. 1997. A partial generic revision of Campylium (Musci). - Bryologist 100: 65-88.

187a. HEDENÄS, L. 1997. The Drepanocladus s. str. species with excurrent costae (Amblystegiaceae). - Nova Hedwigia 64(3-4): 535-547.

187b. HEDENÄS, L. 1998. An overview of the Drepanocladus sendtneri complex. - J. Bryol. 20: 83-102.

188. HEDENÄS, L. 2006. Additional insights into the phylogeny of Calliergon, Loeskypnum, Straminergon, and Warnstorfia (Bryophyta: Calliergonaceae). - J. Hattori Bot. Lab. 100: 125-134.

189. HEDENÄS, L. \& N. PEDERSEN 2002. Nomenclatural consequences of a phylogenetic study of the Plagiotheciaceae. - Bryologist 105: 325-326.

190. HILL, M.O., N. BELL, M.A. BRUGGEMAN-NANNENGA, M. BRUGUÉS, M.J. CANO, J. ENROTH, K.I. FLATBERG, J.-P. FRAHM, M.T. GALLEGO, R. GARILLETI, J. GUERRA, L. HEDENAS, D.T. HOLYOAK, J. HYVÖNEN, M.S. IGNATOV, F. LARA, V. MAZIMPAKA, J. MUÑOZ \& L. SÖDERSTRÖM 2006. An annotated checklist of the mosses of Europe and Macaronesia. J. Bryol. 28: 198-267.

191. HOFFMANN, H. 1998. A monograph of the genus Homalothecium (Brachytheciaceae, Musci). - Lindbergia 23: $119-159$.

192. HOLYOAK, D.T. 2004. Taxonomic notes on some European species of Bryum (Bryopsida: Bryaceae). - J. Bryol. 26: 247-264.

193. HORTON, D.G. 1983. A revision of the Encalyptaceae (Musci), with particular reference to the North American taxa. Part 2. - J. Hattori Bot. Lab. 54: 353-532.

194. HUTTUNEN, S., A. GARDINER \& M.S. IGNATOV 2006. Additional comments on the phylogeny of the Brachytheciaceae (Bryophyta). - In: Newton, A.E. \& R. Tangney (eds.), Pleurocarpous mosses: systematics and evolution. CRC Press, Bocan Rota (Florida): 111-137.

195. HYVÖNEN, J. 1989. A synopsis of the genus Pogonatum (Polytrichaceae, Musci). - Acta Bot. Fenn. 138: 1-87.

196. IGNATOV, M.S. 1992. On the occurrence of Barbula inaequalifolia Tayl. (Pottiaceae, Musci) in Altai Mountains. - Укр. Бот. Журн. [Ukr. Bot. Zhurn.] 49(1): 95-97.

197. IGNATOV, M.S. 1994. Bryophytes of Altai Mountains. I. Study area and history of its bryological exploration. Arctoa 3: 13-27.

198. IGNATOV, M.S. 1998. Bryophyte flora of Altai Mountains. VIII. Brachytheciaceae. - Arctoa 7: 85-152. 
199. IGNATOV, M.S. \& O.M. AFONINA (eds.) 1992. Checklist of mosses of the former USSR. - Arctoa 1: 1-85.

200. IGNATOV, M.S., H. ANDO \& E.A. IGNATOVA 1996. Bryophyte flora of Altai Mountains. VII. Hypnaceae and related pleurocarps with bi- or ecostate leaves. - Arctoa 6: 21-112.

201. IGNATOV, M., A. BERSANOVA, Z. KHARZINOV \& E. IGNATOVA 2005 [2006] Leptodontium (Pottiaceae, Bryophyta), a new genus for Caucasus. - Arctoa 14: 35-38.

202. IGNATOV, M.S. \& CAO TONG 1994. Bryophytes of Altai Mountains. IV. The family Grimmiaceae (Musci). Arctoa 3: 67-122.

203. IGNATOV, M.S. \& V.Ya. CZERDANTSEVA 1995. The families Cryphaeaceae, Leucodontaceae and Leptodontaceae (Musci) in Russia. - Arctoa 4: 65-104.

204. IGNATOV, M., A. GARDINER, V. BOBROVA, I. MILYUTINA, S. HUTTUNEN \& A. TROITSKY 2006. On relationships of mosses of the order Hypnales, with the special reference to taxa traditionally classified in Leskeaceae. - In: Newton, A.E. \& R. Tangney (eds.), Pleurocarpous mosses: systematics and evolution. CRC Press, Bocan Rota (Florida): 171-207.

205. IGNATOV, M.S. \& S. HUTTUNEN 2002. Brachytheciaceae (Bryophyta) - a family of sibling genera. - Arctoa 11: $245-296$

205a. [IGNATOV, M.S. \& E.A. IGNATOVA] ИГНАТОВ M.С., Е.А. ИГНАТОВА 1989. Мхи Холодной речки (Абхазская CCP). - [Mosses of Kholodnaya Reczka (Abkhazskaya SSR)] Бюлл. Гл. Бот. Сада [Byull. Galavnogo Bot. Sada] 152: 63-67.

206. IGNATOV, M.S. \& E.A. IGNATOVA 2003. Brachythecium buchananii (Brachytheciaceae, Musci) - a new species for Uzbekistan. - Arctoa 12: 113-114.

207. [IGNATOV, M.S. \& E.A. IGNATOVA] ИГНАТОВ М.C., Е.А. ИГНАТОВА 2003-2004. Флора мхов средней части европейской России. Т. 1-2. - [Moss flora of the Middle European Russia. Vols. 1-2] M., KMK [Moscow, KMK]: 1 (2003): 1-608; 2 (2004): 609-960.

208. IGNATOV, M.S., E.A. IGNATOVA, T.V. AKATOVA \& N.A. KONSTANTINOVA 2002. Bryophytes of the Khosta' Taxus and Buxus Forest (Western Caucasus, Russia). Arctoa 11: 205-214.

209. IGNATOV, M.S., E.A. IGNATOVA \& V.Ya. CHERDANTSEVA 2006. Oedipodium griffithianum (Dicks.) Schwägr. (Oedipodiopsida, Bryophyta), a new species and class for Russia. - Arctoa 15: 211-214.

210. IGNATOV, M.S., E.A. IGNATOVA, Z. IWATSUKI \& B.C. TAN 1999. Two new moss taxa from the Bureya River, Russian Far East. - Arctoa 8: 59-68.

211. IGNATOV, M.S., E.A. IGNATOVA \& N.A. KONSTANTINOVA 2005. Bryophyte flora of the Volzhsko-Kamskiy Nature Reserve (Tatarstan, European Russia). - Arctoa (2005) 14: 49-66.

212. [IGNATOV, M. S., E. A. IGNATOVA \& G. A. PRONKINA] ИГНАТОВ М.C., Е.А. ИГНАТОВА, Г.А. ПРОНЬКИНА. 2004. Мхи заповедников России. [Mosses of State Reserves of Russia] Современное состояние биологического разнообразия на запо- ведных территориях России. Том. 3. Лишайники и мохообразные. М., МСОП [Sovremennoe sostoyanie biologicheskogo raznoobraziya na zapovednykh territoriyakh Rossii. Vol. 3. Lishainiki i mokhoobraznye. Moscow, IUCN]: 274-366.

213. IGNATOV, M.S., E.A. IGNATOVA \& S.A. SURAGINA 2002. A new variety of Syntrichia caninervis (Pottiaceae, Musci). - Arctoa 11: 333-336.

214. IGNATOV, M.S. \& J. LEWINSKY-HAAPASAARI 1994. Bryophytes of Altai Mountains. II. The genera Amphidium Schimp., Orthotrichum Hedw. and Zygodon Hook. \& Tayl. (Orthotrichaceae, Musci). - Arctoa 3: 29-57.

215. IGNATOV, M.S., I.A. MILYUTINA \& S. HUTTUNEN 2006. On two East Asian species of Brachythecium (Brachytheciaceae, Musci). - J. Hattori Bot. Lab. 100: 191-199.

216. IGNATOV, M.S., I.A. MILYUTINA, T. KOPONEN, D.C. LONG \& E. IGNATOVA 2006. Taxonomy of Struckia (Plagiotheciaceae, Bryophyta) based on molecular and morphological data. - Chenia, Vol. 9.

217. IGNATOV, M.S. \& R. OCHYRA 1994. Bryophytes of Altai Mountains. III. The genus Ulota (Orthotrichaceae, Musci). - Arctoa 3: 59-66.

218. IGNATOV, M.S. \& R. OCHYRA 1995. On the systematic position of Myurella and Bardunovia, genus novus (Plagiotheciaceae, Musci). - Arctoa 5: 45-59.

219. IGNATOV, M.S. \& G.L. SMITH MERRILL 1995. Bryophytes of Altai Mountains. VI. The Family Polytrichaceae (Musci). - Arctoa 5: 61-97.

220. IGNATOV, M.S. \& B.C. TAN 1991. Orthodontopsis, a new genus of Bryaceae (Musci) from southern Siberia, USSR. - J. Hattori Bot. Lab. 71: 165-173.

221. IGNATOV M.S., B.C. TAN, Z. IWATSUKI \& E.A. IGNATOVA 2000. Moss flora of the Upper Bureya River (Russian Far East). - J. Hattori Bot. Lab. 88: 147-178.

222. IGNATOV, M.S. \& R.H. ZANDER 1993. Barbula amplexifolia from the Altai Mountains of Russia. - Bryologist 96(4): 638-639.

223. IGNATOVA, E.A. 2001. A new species of Barbula (Pottiaceae, Musci) from Siberia. - Arctoa 10: 161-164.

224. [IGNATOVA, E.A.] ИГНАТОВА Е.A. 2005. О распространении видов Dicranum с трубчато свернутыми листьями в России. - [On the distribution of Dicranum species with tubulose leaves in Russia] $B$ кн.: «Актуальные проблемы бриологии», Тр. междунар. сов. посв. 90-летию со дня рожд. А.Л. Абрамовой. СПб, 2225 ноября 2005. [In: Proc. Int. Conf. “Actual Problems of Bryology" devoted to 90 ${ }^{\text {th }}$ Anniversary of A.L. Abramova. St. Petersburg, 22-25 November 2005]: 95-101.

225. IGNATOVA, E., H. BEDNAREK-OCHYRA, O. AFONINA \& J. MUÑOZ 2003 [2004]. A new species of Grimmia (Grimmiaceae, Musci) from north-east Asia and Alaska. - Arctoa 12: 1-8.

226. [IGNATOVA, E.A. \& V.B. GOLUB] ИГНАТОВA Е.A., В.Б. ГОЛУБ 2006. Новые находки мхов в Краснодарском крае. 1. - [New moss records from Krasnodar Territory. 1] Arctoa 15: 256.

227. IGNATOVA, E.A. \& M.S. IGNATOV 2001. Bryoeryth- 
rophyllum ferruginascens (Stirt.) Giac. (Pottiaceae, Musci) in Russia. - Arctoa 10: 151-154.

228. IGNATOVA, E.A. \& M.S. IGNATOV 2003. Habrodon perpusillus (Habrodontaceae, Musci) - a new family, genus and species for Russia. - Arctoa 12: 133-136.

229. IGNATOVA, E.A. \& M.S. IGNATOV 2005. On the identity of Physcomitrium martianovii (Funariaceae, Bryophyta). - Arctoa 14: 67-70

230. [IGNATOVA, E.A., M.S. IGNATOV \& A.G. BEZGODOV] ИГНАTOBA Е.A., М.С. ИГНАТОВ, А.Г. БЕЗГОДОВ 1996. Мхи Вишерского заповедника (Пермская область, Северный Урал). - [Mosses of the Vishera State Reserve (Perm Province, Northern Ural Mountains)] Arctoa 6: 7-19.

231. [IGNATOVA, E.A., M.S. IGNATOV, V.G. ONIPCHENKO, V.I. ZOLOTOV \& N.A. KONSTANTINOVA] ИГНАТОВА Е.А., М.С. ИГНАТОВ, В.Г. ОНИПЧЕНКО, В.И. ЗОЛОТОВ, Н.А. КОНСТАНТИНОВА 2007. Бриофлора Тебердинского заповедника. - [Bryoflora of the Teberda Reserve] Флора и фауна заповедников. Москва [Flora i fauna zapovednikov. Moscow] (in press).

232. IGNATOVA, E.A., M.S. IGNATOV, A.P. SEREGIN, T.V. AKATOVA, N.A. KONSTANTINOVA 2005. Bryophyte flora of the projected Utrish Nature Reserve (North-West Caucasus, Russia). - Arctoa 14: 39-48.

233. IGNATOVA, E., A. MAKSIMOV, T. MAKSIMOVA \& O. BELKINA 2006. Notes on distribution of Schistidium species (Grimmiaceae, Bryophyta) in Murmansk Province and Karelia. - Arctoa 15: 237-247.

234. IGNATOVA, E. \& J. MUÑOZ 2004. The genus Grimmia (Grimmiaceae, Musci) in Russia. - Arctoa 13: 100-182.

235. IGNATOVA, E.A., T.YU. SAMKOVA 2006. Campylopus umbellatus (Arnell) Paris (Leucobryaceae, Bryophyta), a new species for Russia. - Arctoa 15: 215-218.

236. INGERPUU, N., A. KALDA, L. KANNUKENE, H. KRALL, M. LEIS \& K. VELLAK 1994 List of the Estonian bryophytes. - The Naturalist's Notebook 94: 1-175.

237. INGERPUU, N., A. KALDA, L. KANNUKENE, H. KRALL, M. LEIS \& K. VELLAK 1998. Eesti sammalde määraja. - [Handbook of Estonian bryophytes] Tartu, ERMÜ ZBI Eesti Loodusfoto, 239 pp.

238. IVANOVA, E.I., M.S. IGNATOV, I.A. MILYUTINA \& V.K. BOBROVA 2005. On the morphological and molecular differences between Oligotrichum hercynicum and $\mathrm{O}$. falcatum (Polytrichaceae, Bryophyta). - Arctoa 14: 1-11.

239. [IVANOVA, E.I., E.A. IGNATOVA, M.S. IGNATOV, V.I. ZOLOTOV, К.К. KRIVOSHAPKIN] ИВAHOBA Е.И., Е.А. ИГНАТОВА, М.С. ИГНАТОВ, В.И. ЗОЛОТОВ, К.К. КРИВОШАПКИН 2005. Листостебельные мхи. - [Mosses] В кн.: Разнообразие растительного мира Якутии (ред. Н.С.Данилова) Новосибирск, Изд-во СО РАН [In: Danilova, N.S. (ed.), Raznoobrazie rastitelnogo mira Yakutii, Novosibirsk, Sib. Otd. Ross Akad. Nauk]: 105-125.

240. IWATSUKI, Z. \& A. NOGUCHI 1973. Index muscorum japonicarum. - J. Hattori Bot. Lab. 37: 299-418.

241. IWATSUKI, Z. 1991. Catalog of the Mosses of Japan. Nichinan, Hattori Botanical Laboratory, 182 pp.
242. IWATSUKI, Z. 2004. New catalog of the mosses of Japan. - J. Hattori Bot. Lab. 96: 1-182.

243. JIMÉNEZ, J.A. 2006. Taxonomic revision of the genus Didymodon Hedw. (Pottiaceae, Bryophyta) in Europe, North Africa and Southwest and Central Asia - J. Hattori Bot. Lab. 100: 211-292.

244. JUKONIENE, I. 2003. Lietuvos kiminai ir zaliosios samanos. - [Mosses of Lithuania] Vilnius, Botanikos instituto leidykla, $402 \mathrm{pp}$.

245. KANDA, H. 1976 [1977]. A revision of the family Amblystegiaceae of Japan. II. - J. Sci. Hiroshima Univ., ser. B, Div. 2, 16: 47-119.

246. KANNUKENE, L. \& N.V. MATVEJEVA 1996. Mosses from the arctic tundra of the Taimyr Peninsula, Siberia. Proc. of Estonian Acad. Sci. Biol. 45(1/2): 51-67.

247. [KAZANOVSKY, S.G.] КАЗАНОВСКИЙ С.Г. 1991. К бриофлоре Байкальского заповедника. - [On the bryoflora of Baikal Reserve]. Бриология в СССР, ее достижения и перспективы (Мат. конф., Львов, 10-12 сент. 1991) (ред. Демкив, О. Т.), Львов, АН СССР, АН УССР [In: Demkiv, O. T. (ed.), Briologia v SSSR, ee dostizheniya i perspektivy (Proc. Conf., Lvov, 10-12 Sept. 1991). Lvov, Akad. Nauk SSSR \& Akad. Nauk Ukr. SSR]: 94-98.

248. KHARZINOV, Z., N. PORTENIER, E. IGNATOVA, S. SHHAGAPSOEV \& M. IGNATOV 2004. Rare species and preliminary list of mosses of the Kabardino-Balkaria (Caucasus). - Arctoa 13: 33-40.

249. [KHARZINOV, Z.Kh., A.N. BERSANOVA, S.H. SHHAGAPSOEV, E.A. IGNATOVA \& M.S. IGNATOV] ХАРЗИНОВ 3.Х., А.Н. БЕРСАНОВА, С.Х. ШХАГАПСОЕВ, Е.А ИГНАТОВА, М.С. ИГНАТОВ 2005. Еще раз об азиатских связях флоры мхов Центрального Кавказа. - [Once more about Asian relationships of moss flora of the Central Caucasus] В кн.: «Актуальные проблемы бриологии», Тр. междунар. сов. посв. 90летию со дня рожд. А.Л. Абрамовой. СПб, 22-25 ноября 2005. [Proc. Int. Conf. "Actual Problems of Bryology" devoted to $90^{\text {th }}$ Anniversary of A.L. Abramova. St. Petersburg, 22-25 November 2005]: 182-188.

250. [KHARZINOV, Z.Kh., M.S. IGNATOV, E.A. IGNATOVA \& N.N. PORTENIER] XAРЗИНOB 3.X., E.A. ИГНАТОВА, М.С. ИГНАТОВ, Н.Н. ПОРТЕНИЕР 2006. Новые находки мхов в Кабардино-Балкарской Республике. 1. - [New moss records from Kabardino-Balkarian Republic. 1] Arctoa 15: 256-258.

251. [KONSTANTINOVA, N.A., A.Yu. LIKHACHEV \& O.A. BELKINA] КОНСТАНТИНОВА Н.А., А.Ю. ЛИХАЧЕВ, О.А. БЕЛКИНА 1993. Дополнения и уточнения к “Конспекту флоры мохообразных Мурманской области". - [Additions and refinements to "Synopsis of the Bryophytes of the Murmansk region"] В кн.: Флористические $и$ геоботанические исследования вМурманской области (ред. Константинова Н.A.), Anamuты [In: Konstantinova, N. A. (ed.), Floristicheskie i geobotanicheskie issledovaniya v Murmanskoj oblasti. Apatity]: 6-44.

252. KOPONEN, T. 1971. A monograph of Plagiomnium sect. Rosulata (Mniaceae). - Ann. Bot. Fennici 8: 305-367.

253. KOPONEN, T. 1996. Notes on Philonotis (Bartrami- 
aceae, Musci). 1. Status and distribution of Philonotis falcata (Hook.) Mitt. - Arctoa 6: 113-117.

253a. KOPONEN, T. 1980. A synopsis of Mniaceae (Bryophyta). IV. Taxa in Europe, Macaronesia, NW Africa and the Near East. - Ann. Bot. Fennici 17: 125-162.

253b. KOPONEN, T. 1994. Cinclidiaceae, Mniaceae and Plagiomniaceae from Minshan Range, northwestern Sichuan, China. - Hikobia 11 (4): 387-406.

253c. KOPONEN, T. \& O.M. AFONINA 1992. Miscellaneous notes on Mniaceae (Bryophyta). XV. Genus Rhizomnium in the Russia east of Ural Mts. - Bryobrothera 1: 245-250.

254. KOPONEN, T. \& I.V. CZERNYADJEVA 2006. Rhizomnium tuomikoskii (Mniaceae, Musci) on the Kamchatka Peninsula, Russian Far East. - Arctoa 15: 183-186.

255. [KOSSOVICH, Е.I.] КОСОВИЧ Е.И. 1989. Находка Tomenthypnum falcifolium (Brachytheciaceae) - нового для флоры Евразии вида. - [Finding of Tomenthypnum falcifolium (Brachytheciaceae) - a new species for Eurasian bryoflora] Бот. журн. [Bot. Zhurn.] 74: 250-253.

256. [KOVALSKY, S.V.] КОВАЛЬСКИЙ С.В. 1998. Рogonatum nanum (Polytrichaceae, Bryopsida) в Средней России. - [Pogonatum nanum (Polytrichaceae, Bryopsida) in Central Russia] Arctoa 7: 83-84.

257. KRAMER, W. 1980. Tortula Hedw. sect. Rurales De Not. (Pottiaceae, Musci) in der Östlichen Holarktis. Bryoph. Bibl. 21: 1-165+29 tabs.

258. KRIVOSHAPKIN, K.K. 1998. Moss flora of the Olekminsky Nature Reserve (Jakutia). - Arctoa 7: 9-20.

259. KRUIJER, H. 2002. Hypopterygiaceae of the world. Blumea, suppl. 13: 1-388.

260. [KURBATOVA, L.Е.] КУРБАТОВА Л.Е. 1998. Род Scouleria Hook. в России. - [Genus Scouleria Hook. in Russia] Новости сист. низи. pacm. [Novosti Sist. Nizsh. Rast.] 32: 162-169.

261. [KURBATOVA, L.E. \& G.YA. DOROSHINA] KУРБAТОВА Л.Е., Г.Я. ДОРОШИНА 2006. Новые находки мхов в Ленинградской области. 1. - [New moss records from Leningrad Province] Arctoa 15: 249.

262. [KURBATOVA, L.E., G.Ya. DOROSHINA-UKRAINSKAYA \& E.O. KUZMINA] КУРБАТОВА Л.Е., Г.Я ДОРОШИНА-УКРАИНСКАЯ, Е.О. КУЗЬМИНА 1999. Листостебельные мхи Ленинградской области. - [Mosses of the Leningrad Province (European Russia)] $B \kappa н$.: Биоразнообразие Ленинградской области (Водоросли. Грибы. Лишайники. Мохообразные. Беспозвоночные животные. Рыбы и рыбообразные) (ред. Балашова Н.Б., А.А. Заварзин), СПб, изд-во СПб ун-та [In: Balashova N.B., A.A. Zavarzin (eds.), Biodiversity of Leningrad Province (Algae, fungi, lichens, bryophytes, invertebrates, fishes and pisciformes) St.-Petersburg, Izd. SPb. Univ.]: 271-302.

263. [KUVAEAV, V.В.] КУВАЕВ В.Б. 1996. К флоре листостебельных мхов острова Сибирякова. - [On moss flora Sibiryakov Island] Новости сист. низи. pacm. [Novosti Sist. Nizsh. Rast.] 31: 167-170.

264. [KUZMINA, E. YU \& I.V. CZERNYADJEVA] КУЗЬМИНА Е.Ю., И.В. ЧЕРНЯДЬЕВА 2005. Листостебельные мхи бассейна р. Сабун (Среднее течение р. Обь, Западная Сибирь). - [Mosses of Sabun River basin, middle course of Ob River, West Siberia] Новости сист. низи. pacm. [Novosti Sist. Nizsh. Rast.] 38: 340-356.

265. LAPSHINA, E.D. \& E.Ya. MULDIYAROV 1998. The bryophyte flora of the Middle Western Siberia. - Arctoa 7: 25-32.

266. [LAPSHINA, E.D.] ЛАПШИНА Е.Д. 2003. Флора болот юго-востока Западной Сибири. - [Flora of mires of southern-east West Siberia] Томск, изд.-во Томского Унив. [Tomsk, izd. Tomsk Univ.]: 1-296.

267. [LAZARENKO, A.S.] ЛАЗАРЕНКО А.C. 1938. Матеріали до бріофлори Середньоі Азіi. - [Materials on brioflora of Middle Asia] Журн. Інст. Бот. АН УРСР [Zhurn. Inst. Bot. Akad. Nauk Ukr.SSR] 26-67: 191-216.

268. [LAZARENKO, A.S.] ЛАЗАРЕНКО А.С. 1940-1945. Листяні мохи Радянського Далекого Сходу. I-IV. [Mosses of the Soviet Far East. I-IV]. Бот. Журн. АH YPCP [Bot. Zhurn. Akad. Nauk Ukr. RSR] Pt. I (1940): 1(3-4): 59-100; Pt. II (1941): 2(1): 51-95; Pt. III (1941): 2(2): 271-308; Pt. IV (1945): 2(3-4): 185-216.

269. [LAZARENKO, A.S.] ЛАЗАРЕНКО А.C. 1955 Определитель лиственных мхов Украины. - [Handbook of mosses of Ukraine] Киев, Изд-во АН УССР [Kiev, Izd. Akad. Nauk UkrSSR], 468 pp

270. LEWINSKY, J. 1993. A synopsis of the genus Orthotrichum Hedw. (Musci, Orthotrichaceae). - Bryobrothera 2: 1-59.

271. LEWINSKY-HAAPASAARI, J. 1994. Miscellaneous notes on Orthotrichum 5. Orthotrichum vicarium Laz. Lindbergia 19: 37-39.

272. LEWINSKY-HAAPASAARI, J. 1996. Orthotrichum holmenii, a new corticolous species from Kazakhstan with comments on Orthotrichum hallii in Asia. - Bryologist 99: 1-5.

273. [LIKHACHEV, А.Yu. \& O.A. BELKINA] ЛИХАЧЕВ А.Ю., О.А. БЕЛКИНА 1999. Листостебельные мхи горного массива Лавна-тундра (Мурманская область, Россия). - [Mosses of Lavna-Tundra mountains (Murmansk Province, Russia)] Arctoa 8: 5-16.

274. LINDBERG, S.O. 1872. Contributio ad floram cryptogamam Asiae boreali-orientalis. - Acta Soc. Sci. Fennicae 10: $221-280$.

275. LINDBERG, S.O. \& H.W.ARNELL 1890. Musci Asiae Borealis. - Kongl. Svenska Vetensk.-Akad. Handl. 23(10): 1-163.

276. LÜTH, M. 2006. Neue Moosfunde aus Sudbaden und Bemerkungen zu einigen kritischen Arten. - Herzogia 19: 323-339.

277. [LYUBARSKAYA, L.B.] ЛЮБАРСКАЯ Л.Б. 1986. Конспект флоры листостебельных мхов Азербайджана. - [Conspect of mosses of Azerbaijan] Баку, Инст.Бот. AН АзССР, рукопись деп в ВИНИТИ [Baku, Inst. Bot. Akad. Nauk Azerb. SSR (msc)], 178 pp.

278. MAGILL, R.E. \& V.Ya. CHERDANTSEVA 1995. Meteorium (Musci, Meteoriaceae) in the Russian Far East. Fragm. Florist. et Geobot. 40(1): 223-227.

279. MAIER, E. 1998. Zur systematischen Stellung von Grimmia pitardii Corb. (Musci). - Candollea 53: 301-308.

280. MAKSIMOV, A. 1995. New data on the distribution of Sphagnum molle in Karelia (Russia). - Memoranda Soc. 
Fauna Flora Fennica 71(1-2): 1-2.

281. [MAKSIMOV, А.I.] МАКСИМОВ А.И. 2000. Редкие листостебельные мхи Карелии. - [The rare mosses of Karelia (Russia)] Бот. Журн. [Bot. Zhurn.] 85(4): 67-80.

282. [MAKSIMOV, A.I.] МАКСИМОВ А.И. 2003. Дополнение к флоре листостебельных мхов национального парка «Паанаярви». - [Additions to moss flora of the Paanajarvi National Park] Труды Карельского НЦРАН. Природа и экосистемы национального парка «Паанаярви». Серия Б. Биология. Bbin. 3. [Proceedings of Karelin Research Centre RAS. Priroda i ecosistemy Natsional'nogo Parka Paanajarvi. Seria B. Biologia] 3: 68-70.

283. [MAKSIMOV, А.І] МАКСИМОВ А.И. 2006. Листостебельные мхи Карелии-[Mosses of Karelia] В кн.: Северная Европа в XXI веке: природа, культура, экономика. Материаль Международной конференции, посвященной 60-летию КарНЦ РАН. Секиия "Биологические науки». Секция «Науки о земле». Петрозаводск, Карельский НЦ PAH [In: Severnaya Evropa v XXI veke: priroda, kul'tura, ekonomika. Materialy Mezhdunarodnoj konferentsii, posvyashchennoj 60-letiyu Karelian Recearch Centre RAS. Sektsiya "Biologicheskie nauki". Sektsiya "Nauki o zemle". Petrozavodsk, Karelian Research Centre]: 140-142.

284. [MAKSIMOV, A.I. \& E.I. IVANOVA] МАКСИМОВ А.И., Е.И. ИВАНОВА 2006. Сфагновые мхи низовьев реки Индигирка. - [Sphagnum mosses in the Lower of Indigirka River] Биоразнообразие растительного покрова Крайнего севера: инвентаризация, мониторинг, охрана. Материаль Всероссийской конференции. Cыктывкар [Bioraznoobrazie rastitel'nogo pokrova Krainego Severa: inventarizatsia, monitoring, okhrana. Materialy Vserossijskoj konferentsii, Syktyvkar]: 67-68.

285. [MAKSIMOV, A.I. \& T.A. MAKSIMOVA] МАКСИМОВ А.И., Т.А. МАКСИМОВА 1998. Первая находка Fissidens pusillus (Fissidentaceae, Musci) в Карелии [The first record of Fissidens pusillus (Fissidentaceae, Musci) in Karelia] Бот. журн. [Bot. Zhurn.] 83 (6): 123-127.

286. [MAKSIMOV, A.I. \& T.A. MAKSIMOVA] МАКСИМОВ А.И., Т. А. МАКСИМОВА 2001. 3.2. Листостебельные мхи - [3.2. Mosses] Инвентаризачия и изучение биологического разнообразия на территории центральной Карелии (оперативно-информационные материаль). Петрозаводск: Карельский научный центр РАН. [Invеntarizatsiya i izuchenie biologicheskogo raznoobraziya na territorii central'noj Karelii (operativno-informatsionnye materialy). Petrozavodsk, Karelian Research Centre]: 94-101.

287. [MAKSIMOV, A.I. \& T.A. MAKSIMOVA] МАКСИМОВ А.И., Т.А. МАКСИМОВА 2003. 4.2. Листостебельные мхи - [4.2. Mosses] В кн.:Материалы инвентаризации природных комплексов и научное обоснование ландиафтного заказника «Сыроватка». (ред. А.Н. Громиев). Петрозаводск [In: Gromtsev, A. N. (ed.), Materialy inventarizatsii prirodnykh kompleksov $i$ nauchnoe obosnovanie landshaftnogo zakaznika "Syrovatka". Petrozavodsk]: 46-50.

288. [MAKSIMOV, A.I. \& T.A. MAKSIMOVA] МАКСИМОВ А.И., Т.А. МАКСИМОВА. 2005а. Материалы к флоре листостебельных мхов планируемого природного парка «Кожозерский» (Архангельская область). - [Note to moss flora of the proposed Kozhozerskij National Park
(Archangelsk district)] Труды Карельского НЦ РАН. Биогеография Карелии. Петрозаводск [Proceedings of Karelian Research Centre RAS. Biogeografiya Karelii. Petrozavodsk] 7: 157-167.

289. [MAKSIMOV, A.I. \& T.A. MAKSIMOVA] МАКСИМОВ А.И., Т.А. МАКСИМОВА. 2005b. Листостебельные мхи. - [Mosses]. В кн.: Природные комплексы Вепсской волости: особенности, современное состояние, охрана и использование (Ред. А.Н. Громцев). Карельский НЦ РАН. Петрозаводск [In: Gromtsev (ed), Prirodnye kompleksy Vepsskoij volosti, sovremennoe sostoyanie, okhrana i ispol'zovanie. Petrozavodsk, Karelia Research Centre RAS]:127-134.

290. [MAKSIMOV, A.I. \& T.A. MAKSIMOVA] МАКСИМОВ А.И., Т.А. МАКСИМОВА 2006. К флоре листостебельных мхов бывшего национального парка Хиисъярви и его окрестностей (Карелия) - [Note to moss flora of the Former Hiisjarvi National Park and their vicinity] Устойчивость экосистем и проблема сохранения биоразнообразия на Севере. Материаль Международной конференции. Кировск. [Ustoichivost' ekosistem i problemy sokhranenia bioraznoobraziya na Severe. Materialy Mezhdunarodnoj konferentsii. Kirovsk] 1: 116-119.

291. MAKSIMOV, A.I., T.A. MAKSIMOVA \& M.A. BOICHUK. 2003. 3.2. Mosses in protected areas. - In: Gromtsev A. N. \& al., eds. Biotic diversity of Karelia: conditions of formation, communities and species. Petrozavodsk: Karelian Research Centre of RAS: 89-102.

292. [MAKSIMOV, A.I., L.A. VOLKOVA \& I.V. KUKSA] МАКСИМОВ А.И., Л.А. ВОЛКОВА, И.В. КУКСА 1995. Листостебельные мхи заповедника "Кивач". - [Mosses of the Kivach Reserve] В кн.: Флористические исследования в Карелии. Bып. 2. Петрозаводск [In: Floristicheskie issledovaniya v Karelii. Pt. 2. Petrozavodsk]: 43-67.

293. [MАМАTKULOV, U.К.] МАМАТКУЛОВ У.К. 1967. Мхи заповедника «Рамит». - [Mosses of the Ramit Reserve] Изв. отд. биол. наук АН ТАдж CCP [Izv. Otd. Biol. Nauk Akad. Nauk Tadjikskoj SSRJ 3(28): 83-86.

294. [MAMATKULOV, U.K.] МАМАТКУЛОВ У.К. 1975. Лиственные мхи Дарвазского хребта. - [Mosses of Darvaz Range] Душанбе, Дониш [Dushanbe, Donish]: 100 pp.

295. [MAMATKULOV, U.K.] МАМАТКУЛОВ У.К. 1990. Флора мохообразных Таджикской ССР. Т. 1. - [The flora of the bryophytes of the Tadjik SSR, Vol. 1] Dushanbe, Donish [Душанбе, Дониш], 320 pp.

296. [MAMATKULOV, U.K., I.O. BAITULIN \& S.G. NESTEROVA] МАМАТКУЛОВ У.К., И.О. БАЙТУЛИН, С.Г. HЕСТЕРОВА 1998. Мохообразные Средней Азии и Казахстана. - [Bryophytes of the Middle Asia and Kazakhstan] Aлмать [Almaty], 232.

297. [MANAKYAN, V.A.] МАНАКЯН В.А. 1995. Итоги бриологических исследований в Армении. - [Results of bryological studies in Armenia] Arctoa 5: 15-33.

298. MATSUI, T. \& Z. IWATSUKI 1990. A taxonomic revision of the family Ditrichaceae (Musci) of Japan, Korea and Taiwan. - J. Hattori Bot. Lab. 68: 317-366.

299. MINAMI, Yo., S. OKITSU, H. KANDA, V.Ya. CHERDANTSEVA \& S.Yu. GRISHIN 2001. Occurrence of 
bryophytes on Paramushir Island, northern Kuriles, Far East Russia. - Mem. Nat. Inst. Polar Res., Spec. Issue 54: 487-493.

300. MOGENSEN, G.S. 1973. A revision of the moss genus Cinclidium Sw. (Mniaceae Mitt.). - Lindbergia 2: 49-80.

301. MOGENSEN, G.S. 2000. Encalypta rhaptocarpa Schwaegr. and E. leptodon Lindb. in Denmark are E. trachymitria Rip.: on their taxonomy and differences (Bryophyta, Musci). - Lindbergia 26: 33-36.

302. MOGENSEN, G.S. \& I. GOLDBERG 2003. The genus Seligeria in the Ural Mountains (Seligeriaceae, Bryophyta). - Lindbergia 28: 59-74.

303. [MONAKHOV, A.K. \& Z.N. SMIRNOVA] MOHAXOB А.К., 3.Н.СМИРНОВА 1968. О находке некоторых северных видов в Центральном Казахстане. - [On the finding of some northern species in the Central Kazakhstan] Новости сист. низи. pacm. [Novosti Sist. Nizsh. Rast.] '1969': 274-278.

304. [MORDVINOV, A.N.] МОРДВИНОВ А.Н. 1994. Бриофлора Жигулевского заповедника. - [The bryoflora of Zigulyovsky Reserve] Бот. журн. [Bot. Zhurn.] 79(4): $65-70$.

305. MUÑOZ, J. 2002 Grimmia exquisita (Musci, Grimmiaceae), a new species from central Asia. - J. Bryol. 24: 315-318.

306. MUÑOZ, J. \& F. PANDO 2000. A world synopsis of the genus Grimmia. - Monogr. Syst. Bot. Missouri Bot. Gard. 83: $133 p p$.

307. [MULDIYAROV, E.Ya. \& N.A. CHERNOVA] МУЛЬДИЯРОВ Е.Я., Н.А. ЧЕРНОВА 2002 [2003]. Новые виды мохообразных Томской области. - [New records of bryophytes in Tomsk Province] Arctoa 11: 215-218.

308. [MULDIYAROV, E.Ya. \& E.D. LAPSHINA] МУЛЬДИЯРОВ Е.Я., Е.Д. ЛАПШИНА 1990. Экологофитоценотическая характеристика бриофлоры района падения Тунгусского метеорита. - [Ecologo-phytocenotic characteristic of bryoflora of the area of the Fall of Tunguska Meteorite]. Следы космических воздействий на землю. Новосибирск, АН СССР, СО, Ин-т геол. и геофиз. Sledy kosmicheskikh vozdeistvij na Zemlyu. Novosibirsk, Akad. NaukSSSR, Sib. Otd. , Inst. Geol. Geofiz.]: 133-139.

309. [NAPREENKO, M.G.] НАПРЕЕНКО М.Г. 2006. Мхи Калининградской области. - [Mosses of Kaliningrad Province], рукопись [manuscript].

310. [NAPREENKO, M.G. \& L.V. RAZGULJAJEVA] НАПРЕЕНКО М.Г., Л.В. РАЗГУЛЯЕВА 1999. СфагНовые мхи Калининградской области. - [Sphagna of Kaliningrad Province (European Russia)] Arctoa 8: 27-34.

311. NEDOLUZHKO, V.A. \& T.A. RUBTSOVA 1998. The first inland locality of Bryoxyphium savatieri (Husn.) Mitt. in the Russian Far East. - Arctoa 7: 79-80.

312. NOGUCHI, A. 1989. Illustrated moss flora of Japan. Pt. 3 - Hattori Botanical Laboratory, Nichinan: 489-742.

313. [NOTOV, A.A., O.M. VOLKOVA, U.N. SPIRINA, L.V. KOLOSOVA \& V.A. RYBKINA] HOTOB A.A., O.M. ВОЛКОВА, У.Н. СПИРИНА, Л.В. КОЛОСОВА, В.А.
РЫБКИНА 2005. О флористическом разнообразии некоторых физико-географических районов Тверской области. - [On floristic diversity of some geographical regions of Tver Province] Вестн. ТвГУ. Сер. биология $и$ экология. Bыn. 1. [Vestnik TvGU. Ser. biologia I ekologia. Vyp. 1$] 4$ (10): 122-150.

314. NYHOLM, E. \& L. HEDENÄS 1986. A new species of Gymnostomum. - Lindbergia 12: 41-42.

315. [NYPORKO, S.O.] НИПОРКО С.О. 2001. Листостеблові мохи природного заповідника «Горгани». [Mosses of the nature reserve "Gorgany"] Укр. Бот. Журн. [Ukr. Bot. Zhurn.] 58(2): 248-255.

316. OCHYRA, R., J. ŻARNOWIEC \& H. BEDNAREKOCHYRA 2003. Census catalogue of Polish mosses. Polish Acad. Sci., Inst. Bot., Krakow: 372 pp.

317. OESAU, A. 2003 Pterygoneurum papillosum (Bryopsida: Pottiaceae), a new moss species from Germany. $-J$. Bryol. 25: 247-252.

318. [ONIPCHENKO, V.G. \& E.A. IGNATOVA] ОНИПЧЕНКО, В.Г., Е.А. ИГНАТОВА 1996. Новые видЫ сосудистых растений и мохообразных для флоры Тебердинского заповедника. - [New vascular plants and bryophytes for the flora of Teberda State Reserve] Бюлл. Моск. о-ва испыт. природы. Отд. биол. [Bull. Mosk. Obshch. Isp. Prir. Otd. Biol.] 101(5): 92-98.

319. OTNYUKOVA, T.N. 1998. Didymodon hedysariformis, a new species of Pottiaceae (Musci) from South Siberia (Tuva Republic, Russia). - Arctoa 7: 206-210.

320. OTNYUKOVA, T.N. 2000. Dicranum orientale (Dicranaceae, Musci), a new species from South Siberia (Tuva Republic, Siberia, Russia). - Бот. Журн. [Bot. Zhurn.] 85(10): 82-87.

321. OTNYUKOVA, T.N. 2001. A new species of Orthotrichum (Orthotrichaceae, Musci) from Tuva Republic, South Siberia. - Arctoa 10: 155-156.

322. OTNYUKOVA, T.N. 2001. Notes on Dicranum (Dicranaceae, Musci) in Russia. 1. Dicranum nipponense found in Far East. - Arctoa 10: 157-160.

323. OTNYUKOVA, T.N. 2002. A study of the Didymodon species (Pottiaceae, Musci) in Russia. I. Species with caducous leaf apices - Arctoa 11: 337-349.

324. [OTNYUKOVA, T.N.] ОТНЮКОВА T.H. 2003. Материалы к флоре листостебельных мхов Тоджинской котловины (республика Тыва, Южная Сибирь). - [Contribution to the moss flora of Todzha Valley (Tyva Republic, South Siberia)] Arctoa 12: 97-109.

325. OTNYUKOVA, T.N., E.A. IGNATOVA, M.S. IGNATOV \& V.E. FEDOSOV 2004. New records of Tortella alpicola Dix. in Eurasia. - Arctoa 13: 197-201.

326. OTNYUKOVA, T.N. \& R. OCHYRA 2003. On $D i-$ cranum dispersum Engelmark and D. orientale Otnyukova. - Arctoa 12: 115-116.

327. OTNYUKOVA, T.N. \& R.H. ZANDER 1998. Didymodon anserinocapitatus, new to Russia from the Yenisey River, South Siberia. - Arctoa 7: 33-35.

328. [PARTYKA, L.Ya.] ПАРТЫКА Л.Я. 2005. Бриофлора Крыма. - [Bryoflora of Crimea] Киев, Институт ботаники 
HAH [Kiev, N.G. Kholodny' Inst. Bot. NAN], 170 pp.

329. [PARTYKA, L.Ya., RAITSI \& K.O. ULYCHNA] ПАРТЫКА, Л.Я., М. РАЙЦІ \& К.О.УЛЫЧНА 1990. Поширення видів роду Rhodobryum (Schimp.) Limpr. на Украіні. - [Distribution of species of the genus Rhodo-

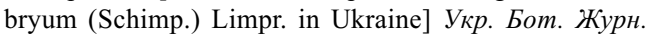
[Ukr. Bot. Zhurn.] 47(3): 28-31.

330. PEDERSEN, N. 2005. Validation of Imbribryum (Bryaceae). - Bryologist 108: 449

331. PEDERSEN, N. \& L. HEDENÄS 2002. Phylogenetic relationships between Bryum and supposedly closely related genera. - J. Bryol. 24: 277-289.

332. PEDERSEN, N. \& L. HEDENÄS 2005. Taxonomic and nomenclatural implications of phylogenetic studies of the Bryaceae based on molecular data and morphology. - Bryologist 108: 123-128.

333. [PIDOPLICHKO, A.P.] ПИДОПЛИЧКО А.П. 1948. Флора сфагновых (торфяных) мхов Белорусской ССР. [Flora of Sphagna of Belorussian SSR] Минск, Изд. Академии наук БССР [Minsk, Izd. Akad. Nauk BSSR], 70 pp.

334. [PISARENKO, O.Yu.] ПИСАРЕНКО, О.Ю. 2001. Mохообразные. - [Bryophytes] $B$ кн: Флора и растительность Катунского заповедника (Горный Алтай) (ред. В.П. Седельников). Новосибирск [In: Sedelnikov, V.P. Flora and vegetation of Katunskii Reserve (Altai mountainous). Nowosibirsk]: 206-227.

335. [PISARENKO, O.Yu.] ПИСАРЕНКО О.Ю. 2004a. Мохообразные - [Bryophytes] В кн.: Флора и растительность Елизаровского государственного заказника (нижняя Объ). Новосибирск. [In: Flora i rastitelnost Elizarovskogo zakaznika (Nizhnyaya Ob). Novosibirsk]: 49-61.

336. PISARENKO, O.Yu. 2004b. Mosses of the central part of Kuznetskiy Alatau (Southern Siberia) - Arctoa 13: 241-260.

337. PISARENKO, O.Yu. 2006a. On the variation and ecology of Pterygoneurum subsessile and P. kozlovii (Pottiaceae, Bryophyta). - Arctoa 15: 169-182.

338. [PISARENKO, O.Yu.] ПИСАРЕНКО О.Ю. 2006 b. Редкие и интересные виды мхов бриофлоры Сибири в гербарии ЦСБС СО РАН. - [Rare and interesting moss species of Siberia in herbarium of Central Siberian Botanical Garden] В кн.: Роль ботанических садов в сохранении биоразнообразия растительного мира Азиатской России: настоящее и будущее. Материаль Всероссийской конференции, посвященной 60-летию Центрального сибирского ботанического сада. Новосибирск [In: Rol botanicheskih sadov v sohranenii bioraznoobrazia rastitelnogo mira Asiatskoj Rossii: nastojaschee i buduschee. (Materiali vserossijskoj konferencii, posviaschennoj 60-letiju Centralnogo Sibirskogo botanicheskogo sada. Novosibirsk]: 218-220.

339. PISARENKO, O.Yu., E.A. IGNATOVA \& M.S IGNATOV 2001. Entostodon hungaricus (Boros) Loeske (Funariaceae, Musci) in Altaisky territory, South Siberia. - Arctoa 10: 97-102.

340. PODPERA, J. 1929. Musci insulae Rossicae prope Vladivostok. - Publ. Fac. Sci. Univ. Masaryk 116: 3-40.

341. PODPERA, J. 1954. Conspectus Muscorum Europaeorum. - Praha, Nakladatelstvi Cesk. Akad. Ved., 699 pp.
342. [POGOSYAN, A.V.] ПОГОСЯН А.В. 2003. Бриофлора вулканического массива Араилер (Республика Армения). - [Bryoflora of volcanic massif Arailer (Republic of Armenia)] Arctoa 12: 187-190.

343. [POPOV, S.Yu., V.E. FEDOSOV, S.A. MOSHKOVSKY \& M. S. IGNATOV] ПОПОВ С.Ю., В.Э. ФЕДОСОВ, С.А. МОШКОВСКИЙ, М.С. ИГНАТОВ 2004 [2005]. Флора мхов Керженского заповедника (Нижегородская область, европейская часть России). - [Moss flora of Kerzhensky State Reserve (Nizhniy Novgorod Province, European Russia)] Arctoa 13: 57-66.

344. [POPOVA, N.N.] ПОПОВА Н.Н. 1988. Конспект мхов. - [Conspect of mosses] В кн.: Цвелев Н.Н. Флора Хоперского государственного заповедника. Л.: Наука [In: Tzvelev, N.N. Flora of Khopersky State Reserve. Leningrad, Nauka]: 173-184.

345. [POРOVA, N.N.] ПОПОВА Н.Н. 2002 [2003]. Бриофлора Среднерусской возвышенности. I. - [Bryoflora of the Central Russian Upland. I] Arctoa 11: 101-168.

346. [PUSHKINA, N.M.] ПУШКИНА Н.M. 1960. Лишайники и мхи Лапландского заповедника. - [Lichens and bryophytes of Laplandsky Reserve] Тр. Лапландск. гос. запка [Trudy Laplandskogo Gos.Zapovednika] 4: 189-248.

347. [RAKHMATULINA, E.K.] РАХМАТУЛИНА Э.К. 1964. Некоторые данные о бриофлоре увлажненных местообитаний западного Тянь-Шаня (средний пояс). - [On the bryoflora of wet habitats of Western Tian-Shan (middle mountain belt)] Узбекск. биолог. журн. [Uzbek. Biol. Zhurn.] 5: 67-70.

348. [RAKHMATULINA, E.K.] РАХМАТУЛИНА Э.К. 1968. Эпифитная флора мхов Бостандыка. - [Epiphyte moss flora of Bostandyk] Узбекск. биолог. журн. [Uzbek. Biol. Zhurn.] 3: 51-54.

349. [RAKHMATULINA, E.K.] РАХМАТУЛИНА Э.К. 1969. К бриофлоре Киргизского хребта (бассейн р. Джарлы-Каиды). - [On bryoflora of Kyrgyzsky Range (Dzharly-Kaida River Basin)] В кн.: Растительные pесурсы Киргизии. Фрунзе [In: Rastitelnye resursy Kyrgyzii. Frunze]: 33-38.

350. [RAKHMATULINA, E.K.] РАХМАТУЛИНА Э.К. 1970. Мхи ельников северного склона хребта Терскей Ала-Toо. - [Mosses of spruce forest of northern slope of Terskei Ala-Toо] Флора Киргизской ССР. Доп. 2. Фрунзе [Flora Kyrgyzskoj SSR. Dopolnenie 2. Frunze]: 50-59.

351. [RAKНMATULINA, E.K.] РАХМАТУЛИНА Э.К. 1990. Мхи гербария института биологии АН Киргизской CCP. - [Mosses of Herbarium of Institute of Biology of Academy of Sciences of Kyrgyz SSR] Известия $A H$ Киргизской ССР, сер. Химико-технологические и биологические науки [Izv. Akad. Nauk Kyrgyzskoj SSR, ser. Khimiko-tekhnologicheskie i biologicheskie nauki] 4 : 48-56.

352. RAZGULYAEVA, L.V., M.G. NAPREENKO, CH. WOLFRAM \& M.S. IGNATOV 2001. Campylopus introflexus (Dicranaceae, Musci) - an addition to the moss flora of Russia. - Arctoa 10: 185-188.

353. REDFEARN, P. L., JR. \& P.-C. WU 1986. Catalog of the mosses of China. - Ann. Missouri Bot. Gard. 73: 177-208. 
354. REDFEARN, P.L., JR., B.C.TAN \& S. HE 1996. A newly updated and annotated checklist of Chinese mosses $-J$. Hattori Bot. Lab. 79: 163-357

355. [RYKOVSKY, G.F. \& O.M. MASLOVSKY] РЫКOBСКИЙ Г.Ф., О.М. МАСЛОВСКИЙ 2004. Флора Беларуси, мохообразные. Том 1 Andreaeopsida-Bryopsida. [Flora of Belarus, Bryophyta. Vol. 1 Andreaeopsida-Bryopsida] Минск, Тэхналогія [Minsk, Taekhnalogia], 439 pp.

356. [SAKAUOVA, G.B.] CAКАУОВА Г.Б. 1992. Мохообразные Южного Алтая. - [Bryophytes of South Altai] Автореф. дисс... канд. биол. наук Душанбе, АН Pесn. Таджикистан, Инст. Бот. [Thesis Ph. D.. Dushanbe, Akad. Nauk. Resp. Tadjikistan, Inst. Bot.], 22.

357. [SAVICZ, L.] САВИЧ Л.И. 1922. Критический обзор новейшей литературы по мхам России (за 1921-1922 г.). - [Critical review of recent literature on mosses, 1921-1922] Изв. Главн. бот. сада [Izv. Glavn. Bot. Sada] 21(2): 1-9.

358. [SAVICZ, L.I.] САВИЧ Л.И. 1936. Материалы к флоре мхов острова Сахалин. - [Contributions to the moss flora of Sakhalin] Вестник ДВФ АН СССР [Vestnik Dalnevostochn. Fil. Akad. Nauk SSSR] 19: 68-85.

359. [SAVICZ-LYUBITSKAYA, L.I.] САВИЧ-ЛЮБИЦКАЯ Л.И. 1954. Обзор рода Bryum Hedw. в СССР. - [An overview of genus Bryum Hedw. in the USSR] Tp. бот. ин-ma AH CССР, сер. 2. Споровые растения [Trudy Bot. Inst. Akad. Nauk SSSR, ser. 2. Sporovye Rasteniya] 9: 495-634.

360. [SAVICZ-LYUBITSKAYA, L.I.] САВИЧ-ЛЮБИЦКАЯ Л.И. 1966. Об изменении названий у некоторых мхов. - [On the name changes of certain mosses] Новости сист. низи. pacm. [Novosti Sist. Nizsh. Rast.]'1966': 330-332.

361. [SAVICZ-LYUBITSKAYA, L.I. \& Z.N. SMIRNOVA] САВИЧ-ЛЮБИЦКАЯ Л.И., З.Н. СМИРНОВА 1970. Определитель листостебельных мхов CCCP. Верхоплодные мхи. - [Handbook of mosses of the USSR. The acrocarpous mosses] Л., Наука [Leningrad, Nauka], 822.

362. [SCHLJAKOV, R.N.] ШЛЯКОВ Р.H. 1951. Два новых вида мхов. - [Two new bryophyte species] Бот. матер. Omd. cnop. pacm. Бот. ин-ma AH CCCP [Bot. Mat. Otd. Spor. Rast. Bot. Inst. Akad. Nauk SSSR] 7: 227-234.

363. [SCHLJAKOV, R.N.] ШЛЯКОВ Р.H. 1999. Новые названия некоторых таксонов мхов России. - [The new names of some taxons of Russian mosses] Новости сист. низи. pacm. [Novosti Sist. Nizch. Rast.] 33: 196-198.

364. [SCHLYAKOV, R.N. \& N.A. KONSTANTINOVA] ШЛЯКОВ Р.Н., Н.А. КОНСТАНТИНОВА 1982. Конспект флоры мохообразных Мурманской области. [Conspectus of bryophyte flora of Murmansk Province] Anатиты, Полярно-Альпийский бот. сад [Apatity, Polar-alpine Bot. Gard.], 228 pp.

365. [SEMENOV, B.S.] CEMEHOB Б.C. 1929. Сфагны Алтая. - [Sphagna of Altai] Труды Алтайского подотдела Русск. Геогр. O-ва [Trudy Altaisk. Pod. Russ. Geogr. Obsc.] 1: 1-44.

366. [SEREDA, V.A.] СЕРЕДА В.А. 2006. Новые находки мхов в Ростовской области. 1. - [New moss records from Rostov-na-Donu Province] Arctoa 15: 255-256.

366a. [SHUBINA T.P. \& G.V. ZHELEZNOVA] ШУБИНА Т.П., Г.В. ЖЕЛЕЗНОВА 2002. Листостебельные мхи равнинной части средней тайги европейского СевероВостока. - [ Mosses of the plain landscapes of the middle taiga of the European North-East] Екатеринбург: УрO PAH [Ekaterinburg, UrO RAN]: 157 pp..

367. [SIMONOV, G.P.] СИМОНОВ Г.П. 1978. Определитель листостебельных мхов Молдавской CCP. [Handbook of mosses of Moldavian SSR] Кишинев, Штиинца [Kishinev, Schtiinza], $168 \mathrm{pp}$.

368. [SIROTINA, I.V.] СИРОТИНА И.В. 1987. Мхи Копетдага. - [Mosses of Kopet Dagh] Дис. ... канд. биол. Наук. Л. БИН АН СССР [Ph. D. Thesis. Leningrad, Botanical Inst. Akad. Nauk SSSR], 191 pp.

369. [SMIRNOVA, Z.N.] СМИРНОВA 3.Н. 1952. Новый вид рода Drepanocladus с Курильских островов. - [New species of the genus Drepanocladus from Kuril Islands] Бот. мат. Отд. споровых раст. Бот. ин-та АН СССР [Bot. Mat. Otd. Spor. Rast. Bot. Inst. Akad. Nauk SSSR] 8: 210-213.

370. [SMIRNOVA, Z.N.] СМИРНОВА 3.H. 1966. О Mnium andrewsianum Steere в CCCP. - [On Mnium andrewsianum Steere in USSR] Новости сист. низи. раст. [Novosti Sist. Nizsh. Rast.] '1966': 332-338.

371. SMITH, A.J.E. 2004. The moss flora of Britain and Ireland. 2 ed. - Cambridge, Cambridge University Press, 1012 pp.

372. SOLDAN, Z. 1991-1992 [1993]. Distribution of Iwatsukiella leucotricha (Musci, Leskeaceae), with notes on a new disjunction in Caucasus. - Novit. Bot. Univ. Carolinae 7: 35-43.

373. [SPIRINA, U.N. \& V.I. ZOLOTOV] СПИРИНА, У.Н., В.И. ЗОЛОТОВ 2004 [2005] Мхи Оренбургского государственного природного заповедника (Юго-восток европейской части России). - [Mosses of the Orenburg State Nature Reserve (South-Eastern European Russia)] Arctoa 13: 51-56.

374. STARK, L.R. 1987. A taxonomic monograph of Forsstroemia Lindb. (Bryopsida: Leptodontaceae). - J. Hattori Bot. Lab. 63: 133-218.

375. STECH, M. 1999. Dichodontium palustre (Dicks.) Stech comb. nov., a new name for Dicranella palustris (Dicks.) Crundw. ex Warb. (Dicranaceae, Bryopsida). - Nova Hedwigia 69: 237-240.

376. SUGAWARA, Sh. 1956. Bryophyta sachalinensis.

377. [SURAGINA, S.A., E.A. IGNATOVA, M.S. IGNATOV \& V.I. ZOLOTOV] СУРАГИНА С.A., Е.А. ИГНАТОВА, М.С. ИГНАТОВ, В.И. ЗОЛОТОВ 2003 '2002'. Материалы к флоре мхов Астраханской области (юг европейской России). - [Contribution to the Moss Flora of Astrakhan Province (South European Russia)] Arctoa 11: 169-174.

378. TAN, B.C. 1991. Miscellaneous notes on Asiatic mosses, especially Malesian Sematophyllaceae (Musci) and others. - J. Hattori Bot. Lab. 70: 91-106.

379. TAN, B.C., W.R. BUCK \& M.S. IGNATOV 1990. On the Hymalayan Struckia C. Muell. and Russian Cephalocladium Lazar. (Musci, Hypnaceae). - Lindbergia 16(3): 100-104.

380. TAN, B.C. \& JIA YU 1999 A preliminary revision of Chinese Sematophyllaceae. - J. Hattori Bot. Lab. 86: 1-70. 
381. [TELEGANOVA V.V. \& M.S. IGNATOV] TEЛEГАНОВА В. В., М.С. ИГНАТОВ 2006. Новые находки мхов в Калужской области. 1. - [New moss records from Kaluga Province. 1] Arctoa 15: 249-250.

382. THÉRIOT, I. 1918. Mousses du Caucase. - Bull. Acad. Int. Géogr., Bot. 28: 96-105.

383. TOUW, A. 2001. A review of the Thuidiaceae (Musci) and a realignment of taxa traditionally accommodated in Thuidium sensu amplo (Thuidium Schimp., Thuidiopsis (Broth.) M. Fleisch., and Pelekium Mitt.) including Aequatoriella gen. nov., and Indothuidium gen. nov. - J. Hattori Bot. Lab. 90: 167-209.

384. TOWNSEND, C.C. 1991. Two mosses of interest in the Soviet Union. - J. Bryol. 16: 648-649.

385. TOWNSEND, C.C. 1992. Four more interesting mosses in Uzbekistan. - J. Bryol. 17: 374-376.

386. [TUBANOVA, D.Yа.] ТУБАНОВА Д.Я. 2004. Мхи Джергинского заповедника. - [Mosses of Dzherginsky Reserve] B cm.: Игнатов М.С., Е.А.Игнатова, Г.А. Пронькина, Мхи заповедников России. Современное состояние биологического разнообразия на заповедных территориях России. Том. 3. Лишайники и мохообразные. М., МСОП [In: Ignatov, M.S., E.A. Ignatova \& G.A. Pronkina, Mosses of State Reserevs of Russia. Sovremennoe sostoyanie biologicheskogo raznoobraziya na zapovednykh territoriyakh Rossii. Vol. 3. Lishainiki $i$ mokhoobraznye. Moscow, IUCN]: 274-366.

387. TUBANOVA D.YA., O.A. ANENKHONOV 2004. The epiphytic mosses in Northern Buryatia (Eastern Siberia). - Arctoa 13: 85-88.

388. [TUBANOVA, D.YA., E.A. IGNATOVA \& V.I. ZOLOTOV] ТУБАНОВА Д.Я., Е.А. ИГНАТОВА, В.И. ЗОЛОТОВ 2006. Новые находки мхов в Республике Бурятия. 1. - [New moss records from Republic Buryatiya. 1] Arctoa 15: 261-263.

389. [UKRAINSKAYA, G.Үа.] УКРАИНСКАЯ Г.Я. 1996а. О сходстве и различии Plagiothecium piliferum (Sw.) Bruch et al. и Isopterygiopsis muelleriana (Schimp.) Iwats. - [On the similarity and difference of Plagiothecium piliferum (Sw.) Bruch et al. and Isopterygiopsis muelleriana (Schimp.) Iwats.] Новости сист. низи. pacm. [Novosti Sist. Nizsh. Rast.] 31: 185-191.

390. [UKRAINSKAYA, G.Yа.] УКРАИНСКАЯ Г.Я. 1996b. Plagiothecium berggrenianum (Plagiotheciaceae, Musci) в России. - [Plagiothecium berggrenianum (Plagiotheciaceae, Musci) in Russia] Бот. Журн. [Bot. Zhurn.] 81(2): 87-91.

391. ULVINEN, T. 1996. Bryophytes of the former Kutsa Nature Reserve. - Oulanka Reports 16: 53-62.

392. [ULYCHNA, K.O. \& N.M. VORONINA] УЛЫЧНА К.О., Н.М. ВОРОНИНА 1978 [1979]. Листяні мохи. 3. - [Mosses. 3] Каталог музейных фондов. Збірник наукових праць Львів. прир. муз. Киів [In: Katalog muzeinykh fondov. Zbirnik naukovikh praz Lvivskogo Pripodnogo Muzeya. Kyiv]: 4-18.

393. [UTEKHIN, V.D.] УТЕХИН В.Д. 1976. К флоре зеленых мхов заповедника Аксу-Джабаглы. - [On moss flora of Aksu-Dzhabagly Reserve] В кн.: Заповеднику Аксу-Джабаглы 50 лет. Алма-Ama [In: Zapovedniku
Aksu-Dzhabagly 50 let. Alma-Ata]: 56-65.

394. VANDERPOORTEN, A. 2001. The Syntrichia ruralis complex in Belgium. - Cryptogamie, Bryologie 22: 71-84.

395. [VASILYEV, A.N.] ВАСИЛЬЕВ А.Н. 1992. Конспект флоры мохообразных в заповедниках "Столбы" и Саяно-Шушенском. - [Bryophytes of Reserves "Stolby" and Sayano-Shushenskij] Красноярск, Изд-во Красноярск. ун-та [Krasnoyarsk, Izd. Krasnoyarks. Univ.], 112.

396. VELLAK, K., L. KANNUKENE, N. INGERPUU \& M. LEIS 2001. Additions to the list of the Estonian bryophytes, 1997-2001. - Folia Cryptogamica Estonica 38: 71-78.

397. VELLAK, K., N. INGERPUU, L. KANNUKENE \& M. LEIS 2006. New Estonian records. Liverworts and mosses. - Folia Cryptogamica Estonica 42: 107-111.

398. [VIRCHENKO, V.M.] ВІРЧЕНКО В.М 1989. Види секціi Erythrocarpa Kindb. роду Bryum Hedw. у флорі УPCP. - [Species of Bryum sect. Erythrocarpa Kindb. in the flora of Ukraine]. Укр. Бот. Журн. [Ukr. Bot. Zhurn.] 46(5): 51-55.

399. [VIRCHENKO, V.M.] ВIРЧЕНКО В.М. 1992. Чи знайдено Atractylocarpus alpinus (Schimp. ex Milde) Lindb. на Украіні?. - [Is Atractylocarpus alpinus (Schimp. ex Milde) Lindb. found in Ukraine?] Укр. Бот. Журн. [Ukr. Bot. Zhurn.] 49(3): 95-98.

400. [VIRCHENKO, V.M.] ВIРЧЕНКО В.М. 2000. Список бокоплідніх мохів Украіни. - [List of pleurocarpous mosses of Ukraine] Киів, Знання [Kyiv, Znannya], 32 pp.

401. [VIRCHENKO, V.M.] ВIРЧЕНКО В.М. 2001. Список верхоплідніх мохів Украіни. - [List of acrocarpous mosses of Ukraine] Киів, Знання [Kyiv, Znannya], 56 pp.

402. [VIRCHENKO, V.M.] ВIРЧЕНКО В.М. 2004. Нові знахідки рідкісних для Украіни мохоподібних. - [New records of rare bryophyte species in Ukraine] Укр. Бот. Журн. [Ukr. Bot. Zhurn.] 61(1): 106-110.

403. [VIRCHENKO, V.M.] ВІРЧЕНКО В.М. 2005. Рід Zуgodon Hook. et Taylor (Orthotrichaceae, Bryophyta) в Украіні. - [The genus Zygodon Hook. et Taylor (Orthotrichaceae, Bryophyta) in Ukraine] $У_{\kappa p}$. Бот. Журн. [Ukr. Bot. Zhurn.] 62(5): 715-718.

404. [VIRCHENKO, B.M. \& L.A. BABENKO] ВIРЧЕНКО В. М., Л.А. БАБЕНКО 2001. Знахідка Rhynchostegium rotundifolium (Brid.) Bruch et al. та Myuroclada maximoviczii [sic!] (Borszcz.) Steere \& Schof. на сході Європи. - [Finding of Rhynchostegium rotundifolium (Brid.) Bruch et al. and Myuroclada maximoviczii (Borszcz.) Steere \& Schof. in the eastern Europe] $У_{\kappa р}$. бот. журн. [Ukr. Bot. Zhurn.] 58(1): 676-759.

405. [VIRCHENKO, V.M. \& O.O. ORLOV] ВIРЧЕНКО B.M., О.О. ОРЛОВ 2005. Нові та рідкісні мохоподібні для Украінського Полісся. - [New and rare bryophytes for Ukrainian Polessie] Укp. Бот. Журн. [Ukr. Bot. Zhurn.] 62(3): 431-436.

406. [VIRCHENKO, V.M. \& J. VÁŇA] BIРЧЕНКО B.M., I. ВАНЯ 2000. Список печіночників, антоцеротів та сфагнових мохів Украіни. - [List of hepatics, anthocerotes and sphagna of Ukraine] Киів, Знання [Kyiv, Znannya], $31 \mathrm{pp}$. 
407. [VOLKOVA, L.A. \& A.I. MAKSIMOV] ВОЛКОВА Л.А., А.И. МАКСИМОВ 1993. Список листостебельных мхов Карелии. - [Checklist of mosses of the Karelia] В кн.: Растительный мир Карелии и проблемы его охраны (ред. Г А. Елина, А.Д. Волков) Петрозаводск, Карельский научный иентр PAH [In: Elina, G.A. \& A.D. Volkov (eds.), Rastitel'nyj mir Karelii i problemy ego okhrany. Petrozavodsk, Karel'skij nauchnyj tsentr Ross. Akad. Nauk]: 57-91.

408. WARNSTORF, C. 1906. Kryptogamenflora der Mark Brandenburg. Laubmoose. 2 Bd. - Berlin, Verlag von Gebrüder Bornträger, xii +1160 .

409. WERNER, J. 2002. A comparison of Dichodontium flavescens (Dicks.) Lindb. and D. pellucidum (Hedw.) Lindb. (Bryopsida). - J. Bryol. 24: 215-221.

410. WILBRAHAM, J. \& D.G. LONG 2005. Zygodon Hook. \& Taylor and Bryomaltaea Goffinet (Bryopsida: Orthotrichaceae) in the Sino-Himalaya. - J. Bryol. 27: 329-342.

411. ZANDER, R.H.1993. Genera of the Pottiaceae: mosses of harsh environments. - Bull. Buffalo Soc. Nat. Sci. 32: 1-378.

412. [ZEROV, D.K.] ЗЕРОВ Д.К. 1964. Флора печіночних і сфагнових мохів Украіни. - [Flora of hepatics and Sphagna of Ukraina] Киів, Наукова Думка [Kyiv, Naukova Dumka], 356 pp.

413. [ZEROV, D.K. \& L.Ya. PARTYKA] ЗЕРОВ Д.К., Л.Я. ПАРТЫКА 1975. Мохоподібні Украінських Карпат. [Mosses of Ukrainian Carpatians] Киів, Наукова Думка [Kyiv, Naukova Dumka], 231 pp.

414. [ZHELEZNOVA, G.V.] ЖЕЛЕЗНОВА Г.В 2006. Новые находки мхов в республике Коми. 1. - [New moss records from Komi Republic. 1] Arctoa 15: 251-252.

415. ZHELEZNOVA, G.V.] ЖЕЛЕЗНОВА Г.В. 1994. Флора листостебельных мхов Европейского Северо-Востока. - [Moss flora of European North-East] СПб., Наука [St. Petersburg, Nauka], 148.

416. [ZHELEZNOVA, G.V. \& T.P. SHUBINA] ЖЕЛЕ3НОВА Г.В., Т.П. ШУБИНА 1998а. Новые находки мохообразных в Республике Коми (Северо-Восточная Европа). - [New records of bryophytes from Komi Republic (North-East Europe)] Arctoa 7: 189-190.

417. [ZHELEZNOVA, G.V. \& T.P. SHUBINA] ЖЕЛЕ3НОВА Г.В., Т.П. ШУБИНА 1998b. Мохообразные Печоро-Илычского заповедника. - [Bryophytes of Pechoro-Ilych Reserve] Флора и фауна заповедников. Bbin. 65. Москва [Flora i fauna zapovednikov. 65. Moscow]: $34 \mathrm{pp}$.

417a. [ZHELEZNOVA G.V., T.P. SHUBINA] ЖЕЛЕЗНОВA Г.В., Т.П. ШУБИНА 2005. Видовой состав мхов техногенно нарушенных ландшафтов Республики Коми. - [The species composition of mosses in the technogenically disturbed areas of the Komi Republic] Бот. журн. [Bot. Zhurn.] 90(2): 215-222.

418. ZOLOTOV, V.I. 2000. The genus Bryum (Bryaceae, Musci) in the Middle European Russia. - Arctoa 9: 155-232.

419. [ZOLOTOV, V.I.] ЗОЛОТОВ В.И. 2003. Вryum sauterii Bruch et al. в средней части Европейской Росии. [Bryum sauterii Bruch et al. in Middle European Russia] Arctoa 12: 117-120.
420. ZOLOTOV, V.I. 2006a. On systematics and distribution of some species of Bryum (Bryaceae, Bryophyta) in Russia. - Arctoa 15: 155-162.

421. [ZOLOTOV, V.I.] ЗОЛОтОВ В.И. 2006b. Новые находки мхов в Республике Северная Осетия - Алания. 1. - [New moss records from Republic North Ossetiya Alania. 1] Arctoa 15: 256.

422. [ZOLOTOV, V.I. \& E.Z. ВAISHEVA] ЗОЛОТОВ В.И., Э.3. БАИШЕВА 2003. Флора листостебельных мхов заповедника «Шульган-Таш» (Республика Башкортостан, Россия). - [Moss flora of "Shulgan-Tash" nature reserve (Republic Bashkortostan, Russia)] Arctoa 12: 121-132.

423. [ZOLOTOV, V. I. \& N.R. SHAFIGULLINA] ЗОЛОТОВ В. И., Н.Р. ШАФИГУЛЛИНА 2006. Новые находки мхов в республике Татарстан. 1. - [New moss records from Tatarstan Republic. 1] Arctoa 15: 250.

424. "Tortula modica, Irkutsk, Akademgorodok, Bardunov, 10.X.1957; 22.IX.1969 (IRK, sub Pottia truncata)"

425. Hypnum imponens in Caucasus (confirmed by Afonina.): (1) Caucasian Reserve, Guzeripl, beech forest, on rotten trunk, 19.VIII.1928, coll. Z. Smirnova (LE); (2) same, Guzeripl, $1 \mathrm{~km}$ to Goreloe, beech-fir forest, on rotten beech, 28.VII.1935, coll. L.N. Vasilieva (LE); (3) same, near Ternovaya Village, Belaya River, Kisha Creak, alt. 600 m, on remains of beech, 28.VII.1951, coll. Senicheva (LE).

426. "Philonotis falcata, Altai Republic, Chemal, Ignatov, 29/33 (MHA), det T. Koponen."

427. "Fissidens exilis, Altai Territory, Kolyvan, Ignatov, 17.VIII.2004 (MHA)".

428. Sphagnum perfoliatum L.I.Savicz was described with inexact type locality, called just "NW Siberia". There are three collections in LE labelled by Savicz with Notae criticae "Sphagnum perfoliatum nov. sp.", two from Salekhard, or from Novyj Port. Only one of them is labelled with Notae criticae "typus" by Savicz and obviously it has to be designated as the holotype: "Вычегодско-Печерская геоботаническая экспедиция, Н.Я. и С.В.Кац, Sphagnum jamalense Lyd. Savicz spec. nov., окрестности Сале-Харда ЯмалоНенецкого округа, сфагновое болото, в мочежинах 9.VIII.1939 leg. Н. и С. Кац, det. L. Savicz" [VychegodskoPecherskaya Geobotanical Expedition of N.Ya. \& S. V. Katz, vicinity of Salekhard of Yamalo-Nenetsky District, Sphagnum bog, in hollows, 9.VIII.1938 leg. N. \& S. Katz, det. L. Savicz], with Notae criticae 1947: Sphagnum perfoliatum Lyd. Sav. nov. sp. and additional Notae criticae: "Sphagnum perfoliatum (typus)". This locality is outside Arctic according to the present subdivision of Siberia (Fig. 1, page 5 ), although very close to it border.

429. "In plantie Elisabethopoleus, Dr. Kolenai LE".

430. "Hamatocaulis lapponicus, Vologodskaya Province, Totemsky Distr., Nikolaevskaya, 11.VII.1926. O. Gaze \& A. Korchagin (LE)."

431. "Tortula truncata, Altaisky Territory, Barnaul, Ignatova, 10.VI.1988 E.Ignatova (MHA)".

432. "Pseudoleskeella rupestris, Komi Republic, PecheroIlychskiy Nature Reserve, Pechora River Valley, Kameshok $\left(62^{\circ} 03^{\prime} \mathrm{N}-58^{\circ} 13^{\prime} \mathrm{E}\right)$, alt. $200 \mathrm{~m}$, limestone outcrops, on dry tops and ledges, A.G.Bezgodov \& I.B.Kucherov (MW, LE)" 


\section{AUTHORS - ABTOPЫ}

Abolina, Austra - Аустра Адольфовна Аболиня (Аболинь) - Latvian Forestry Institute "Silava", Riga str. 111, Salaspils, LV-2169, Latvia austra@silava.lv

Afonina, Olga.M. - Ольга Михайловна Афонина Ботанический институт им. В.Л.Комарова РАН, ул. Проф. Попова 2, Санкт-Петербург 197376 Россия - Komarov' Botanical Institute of Russian Acad. Sci., Prof. Popova str., 2, Sankt-Petersburg, 197376 Russia

— stereodon@yandex.ru

Akatova, Tatyana V. - Татьяна Владиславовна Акатова - Кавказский государственный природный биосферный заповедник, Майкопское отделение, Советская 187, Майкоп, Республика Адыгея 385000 Россия - Maikop Branch of Caucasian Nature Biosphere Reserve, Sovetskaya str., 187, Maikop, Adygea Republic, 385000 Russia —hookeria@mail.ru

Baisheva, Elvira Z. - Эльвира Закирьяновна Баишева - Институт Биологии Уфимского НЦ РАН, просп. Октября 69, Уфа, Башкортостан 450054 Россия - Institute of Biology of Ufa Science Centre of Russian Academy of Sciences, Oktyabrya 69, Ufa, Bashkortostan, Russia — elvbai@anrb.ru

Bardunov, Leonid V. - Леонид Владимирович Бардунов - Сибирский институт физиологии и биохимии растений, а/я 317, Иркутск 664033 Россия - Siberian Institute of Plant Physiology and Biochemistry, POBox 317, Irkutsk 664033 Russia — herbar@sifibr.irk.ru

Baryakina, Elena A. - Елена Анатольевна Барякина - Институт Ботаники НАН Азербайджана, Потамдарское шоссе 40, Баку, Az-1073 Азербайджан - Institute of Botany of National Academy of Sciences of Azerbaijan, Potamdarskoe Rd., 40, Baku Az-1073 Azerbaijan

—lena2006ba@mail.ru

Belkina, Olga А. - Ольга Александровна Белкина - Полярно-альпийский ботанический садинститут Кольского НЦ РАН, Кировск-6, Мурманская область 184256 Россия - Polar-Alpine Botanical Garden-Institute of Kola Sci. Center of RAS, Kirovsk-6, Murmansk Province 184256 Russia —belkina@aprec.ru

Bezgodov, Andrey G. - Андрей Геннадьевич Безгодов - Россия 614032, Пермь, Маршала Рыбалко 97, кв. 16 - Marshala Rybalko str. 97, kv. 16, Perm, 614032 Russia — fluid@permnipineft.com

Boychuk, Margarita A. - Маргарита Арсеньевна Бойчук - Институт биологии КарНЦ РАН, ул. Пушкинская 11, Петрозаводск 185910 Россия Institute of Biology of Karelian Science Centre of
Russian Academy of Sciences, Pushkinskaya str. 11, Petrozavodsk, 185910 Russia

—boychuk@krc.karelia.ru

Cherdantseva, Valentina Ya. - Валентина Яковлевна Черданцева - Биолого-Почвенный институт ДВО РАН, Проспект Столетия 159, Владивосток 690022 Россия - Institute of Biology and Soil Science of Far Eastern Branch of Russian Acad. Sci., Prospect Stoletiya, 159, Vladivostok 690022 Russia — cryptogamy@ibss.dvo.ru

Czernyadjeva, Irina V. - Ирина Витальевна Чернядьева - Ботанический институт им. В.Л. Комарова РАН, ул. Проф. Попова 2, СанктПетербург 197376 Россия — Komarov' Botanical Institute of Russian Acad. Sci., Prof. Popova str., 2, Sankt-Petersburg, 197376 Russia

— le-bryo@mail.ru

Doroshina, Galina Ya. - Галина Яковлевна Дорошина Ботанический институт им. В.Л.Комарова РАН, ул. Проф. Попова, д. 2, Санкт-Петербург 197376 Россия - Komarov' Botanical Institute, Prof. Popova str., 2, Sankt-Petersburg, 197376 Russia — le-bryo@mail.ru

Dyachenko, Alexander P. - Александр Петрович Дьяченко - Уральский государственный педагогический университет, географо-биологический факультет, просп. Космонавтов, 26, г. Екатеринбург 620017 Россия - GeographicBiological Faculty, Ural's State Pedagogical University, Ekaterinburg 620017 Russia

— eadyach@yandex.ru

Fedosov, Vladimir E. - Владимир Эрнстович Федосов - Биологический факультет, кафедра Геоботаники, МГУ, Москва 119992 Россия Geobotany Dept., Biological Faculty, Moscow State University, Moscow 119992 Russia

— fedosov_v@mail.ru

Goldberg, Irina L. - Ирина Леонидовна Гольдберг - Botanical Garden and Museum, Natural History Museum, University of Copenhagen, Gothersgade 130, DK-1123 Copenhagen K, Denmark — irina.goldberg@gmail.com

Ignatov, Michael S. - Михаил Станиславович Игнатов - Главный ботанический сад РАН Ботаническая 4, Москва127276 Россия - Main Botanical Garden of Russian Acad. Sci., Botanicheskaya 4, Moscow 127276 Russia

_-misha_ignatov@list.ru

Ignatova, Elena A. - Елена Анатольевна Игнатова - Биологический факультет, кафедра Геоботаники, МГУ, Москва 119992 Россия — Geobotany Dept., Biological Faculty, Moscow State University, Moscow 119992 Russia

_-misha_ignatov@list.ru 
Ivanova, Elena I. - Елена Ильинична Иванова Институт биологических проблем криолитозоны СО РАН, Ленина 41, Якутск 677000 Россия - Institute of Biology of Permafrost-Zone of Siberian branch of Russian Academy of Sciences, Lenina str. 41, Yakutsk 677000 Russia

— e.i.ivanova@ibpc.ysn.ru

Jukoniene, Ilona - Илона Юкониене - Institute of Botany, Zaliuju Ezeru 49, LT-08406 Vilnius, Lithuania_ilonet@botanika.lt

Kannukene, Leiti - Лейти Робертовна Каннукене — Estonian Museum of Natural History, 26 Toompuiestee str., 10148 Tallinn, Estonia

—leiti@loodusmuuseum.ee

Kazanovskiy, Sergey G. - Сергей Григорьевич Казановский - Сибирский институт физиологии и биохимии растений, а/я 317, Иркутск 664033 Россия - Siberian Institute of Plant Physiology and Biochemistry, POBox 317, Irkutsk 664033 Russia — kazan@sifibr.irk.ru

Kharzinov, Zaur Kh. - Заур Хасанович Харзинов - Ботанический сад Кабардино-Балкарского гос. университета, Чернышевского 173, Нальчик, Кабардино-Балкарская Республика, 360004 Россия - Botanical Garden of Kabardino-Balkarian State University, Chernyshevskogo, 173, Nalchik360004 Russia — harzinov@rambler.ru

Kurbatova, Lyubov' Е. - Любовь Евгеньевна Курбатова - Ботанический институт им. В.Л.Комарова РАН, ул. Проф. Попова 2, СанктПетербург 197376 Россия — Komarov' Botanical Institute of Russian Acad. Sci., Prof. Popova str., 2, Sankt-Petersburg, 197376 Russia

—1jubov@ak2348.spb.edu

Maksimov, Anatoliy I. - Анатолий Иванович Максимов - Институт биологии КарНЦ РАН, ул. Пушкинская 11, Петрозаводск 185910 Россия - Institute of Biology of Karelian Science Centre of Russian Academy of Sciences, Pushkinskaya str. 11, Petrozavodsk, 185910 Russia

—maksimov_tolya@mail.ru

Maslovsky, Oleg М. - Олег Мечиславович Масловский - Институт экспериментальной ботаники им. В.Ф.Купревича НАН Беларуси, Скорины 27, Минск 220733 Беларусь - V.F. Kuprevich' Institute of experimental botany of National Academy of Scinces of Belarus, Skoriny 27, Minsk 220733 Belarus_oleg@biobel.bas-net.by

Napreenko, Maxim G. - Максим Геннадиевич Напреенко - Калининградский гос. университет, Биологический факультет, кафедра ботаники и физиологии растений, ул. Университетская, 2, Калининград 236040 Россия - Department of Botany and Plant Physiology, Biolog- ical Faculty, Kaliningrad State University, Universitetskaya, 2, Kaliningrad 236040 Russia icid0988@albertina.ru

Otnyukova, Tatyana N. - Татьяна Николаевна Отнюкова - Институт леса им. В.Н.Сукачева СО РАН, Академгородок, Красноярск 660036 Россия - V. N. Sukachev Forest Institute of the Siberian Branch of the Russian Academy of Sciences, Akademgorodok, Krasnoyarsk, 660036 Russia_t totn@ksc.krasn.ru

Partyka, LarisaYa. - Лариса Яківна Партика Інститут ботаніки ім. М. Г. Холодного НАН Украіни, Терещенківська 2, Киів 01601 Украіна - M.H.Kholodny Institute of Botany of National Academy of Sciences of Ukraine, Tereshchenkivska 2, Kyiv 01601 Ukraine

Pisarenko, Olga Yu. - Ольга Юрьевна Писаренко Central Siberian Botanical Garden, Zolotodolinskaya 101, Novosibirsk 630090 Russia - Центральный Сибирский ботанический сад Золотодолинская, 101, Новосибирск 630090 Россия o_pisarenko@mail.ru

Popova, Natalia N. - Наталия Николаевна Попова - Российский государственный социальный университет,Воронежский филиал, ул. Ленинградская, 62, Воронеж 394033 Россия - Russian Social State University, Woronezh Branch, Leningradskaya str., 62, Woronezh 394033 Russia —leskea@vmail.ru

Rykovsky, Gennadiy F. - Геннадий Феодосиевич Рыковский - Институт экспериментальной ботаники им. В.Ф.Купревича НАН Беларуси, Скорины 27, Минск 220733 Беларусь - V.F. Kuprevich' Institute of experimental botany of National Academy of Scinces of Belarus, Skoriny 27, Minsk 220733 Belarus — oleg@biobel.bas-net.by

Tubanova, Dolgor Ya. - Долгор Ямпиловна Тубанова Институт общей и экспериментальной биологии СО РАН, Сахьяновой, д. 6, Улан-Удэ 670047 Россия - Institute of general and experimental Biology of Siberian Branch of Russian Academy of Sciences, Sakhjanovoy str., 6, Ulan-Ude 670047 Russia —tdolgor@mail.ru

Zheleznova, Galina V. - Галина Виссарионовна Железнова Г.В. - Институт биологии Коми НЦ УрО РАН, Коммунистическая 28, Сыктывкар 167610 Россия - Institute of Biology of Komi Science Centre of Ural Branch of Russian Academy of Scinces, Kommunisticheskaya 28, Syktyvkar 167610 Russia — zheleznova@ib.komisc.ru

Zolotov, Valeriy I. - Валерий Иванович Золотов Главный ботанический сад РАН Ботаническая 4, Москва127276 Россия - Main Botanical Garden of Russian Acad. Sci., Botanicheskaya 4, Moscow 127276 Russia — bryum@list.ru 